\title{
Towards Solid-State Spin Based, High-Fidelity Quantum Computation
}

\section{Dissertation}

\author{
for the award of the degree \\ "Doctor rerum naturalium" \\ of the Georg-August-Universität Göttingen \\ within the doctoral program \\ "Physics of Biological and Complex Systems" \\ of the Georg-August University School of Science (GAUSS) \\ submitted by

\section{Felix Kleißler} \\ from Bonn
}

Göttingen 2018 


\section{Members of the Thesis Advisory Committee}

Prof. Dr. Stefan W. Hell (Referee)

Department of NanoBiophotonics

Max Planck Institute for Biophysical Chemistry, Göttingen

Prof. Dr. Marina Bennati (2 ${ }^{\text {nd }}$ Referee)

Max Planck Research Group, Electron-Spin Resonance Spectroscopy

Max Planck Institute for Biophysical Chemistry, Göttingen

Dr. Gopalakrishnan Balasubramanian

Max Planck Research Group, Spin Imaging

Max Planck Institute for Biophysical Chemistry, Göttingen

\section{Members of the Examination Board}

Prof. Dr. Jörg Enderlein

AG Enderlein, Single Molecule Spectroscopy and Imaging for Biophysics and Complex Systems

III. Physical Institute, Georg-August-Universität Göttingen

Prof. Dr. Christian Griesinger

Department of NMR-based Structural Biology

Max Planck Institute for Biophysical Chemistry, Göttingen

Prof. Dr. Martin Uecker

AG Uecker, Cardiovascular Magnetic Resonance Imaging

Institute for diagnostic and interventional radiology, Georg-August-Universität Göttingen

Date of oral examination: 31.08 .2018 
And I'm not happy with all the analyses

that go with just the classical theory,

because nature isn't classical, dammit,

and if you want to make a simulation of nature, you'd better make it quantum mechanical, and by golly it's a wonderful problem, because it doesn't look so easy.

RICHARD FEYNMAN 



\section{Abstract}

This thesis is divided into two parts. Both of them investigate current topics in quantum information processing. The first employs super-resolving stimulated emission depletion (STED) fluorescence microscopy for the characterization of solid-state spins as a resource for quantum information processing. The other focuses on the high-fidelity control of single quantum bits.

In the first part, a custom built STED microscope is utilized to demonstrate imaging of the "Stuttgart 1" (ST1) center with a spatial resolution unlimited by the diffraction of light. The bright fluorescence of the ST1 center in combination with its spin properties make it a promising candidate for quantum information processing and quantum sensing applications. Furthermore, the STED imaging of nitrogen vacancy $(\mathrm{NV})$ center based fluorescent nuclear track detectors is presented. Here, the increased resolution could enable the extraction of the vacancy diffusion coefficient in diamond with high precision. Additionally, an improved understanding of the absorption process of ionizing radiation in matter might by obtained by the imaging of sub-cascade events. While the first is a crucial property in the generation process of NV center based quantum registers, the later is of importance in radiation treatment.

In the second part, a recently proposed universal set of single-qubit superadiabtic geometric quantum gates (SAGQGs) is realized with a fidelity exceeding the error threshold for the efficient implementation of quantum error correction codes. Even though demonstrated for the NV center in diamond, the SAGQG can be realized with any quantum system featuring sufficient control of the driving field parameter. Additionally, a standardized benchmarking analysis is proposed, which identifies the most robust combination of quantum gates for a given set of modalities. It is shown that the most robust universal set of gates is in general not realized by a single quantum gate modality and varies with the physical platform. A systematic application of the benchmarking analysis to currently available noise intermediate scale quantum registers offers the potential to pave the way towards fault-tolerant quantum computation. 



\section{Contents}

$\begin{array}{lr}\text { Introduction } & 1\end{array}$

1. Quantum information processing 3

1.1. Quantum bit operations . . . . . . . . . . . . . . . . . 3

1.1.1. Single qubit quantum gates . . . . . . . . . . . . . . 4



1.1.3. Universal set of gates . . . . . . . . . . . . . . 5



1.3. Quantum error correction codes . . . . . . . . . . . . . . . . . 6

2. Nitrogen vacancy center in diamond $\quad 7$



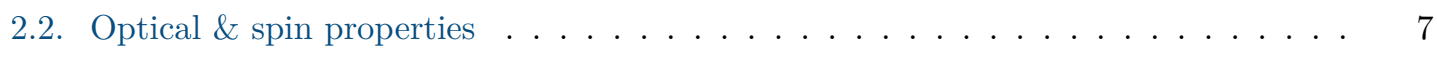



2.4. Current limitations in QIP . . . . . . . . . . . . . . . . . . 10

3. Scope of this thesis 13

$\begin{array}{ll}\text { Super-resolution microscopy } & 15\end{array}$

4. Theoretical background 17

4.1. Fluorescence imaging of solid-state spins . . . . . . . . . . . . . . . . . . . 17

4.1.1. Confocal microscopy . . . . . . . . . . . . . . . . . . . 17

4.1.2. STED microscopy . . . . . . . . . . . . . . . . . 18

4.2. Solid-state nuclear track detection . . . . . . . . . . . . . . . . . . 19

4.2.1. Plastic nuclear track detectors . . . . . . . . . . . . . . . 19

4.2.2. Fluorescent nuclear track detectors . . . . . . . . . . . . . . . . . 20

5. A STED microscope for imaging of solid-state spins $\quad 21$

5.1. Optical configuration . . . . . . . . . . . . . . . . . . 21



5.3. Spatial light modulation . . . . . . . . . . . . . . . 25

5.3.1. Experimental implementation . . . . . . . . . . . . . 25

6. STED imaging of ST1 centers in diamond $r$

6.1. ST1 center properties . . . . . . . . . . . . . . . . 27

6.2. STED imaging . . . . . . . . . . . . . . . . . . . . . 28

6.2.1. Comparison to the NV center . . . . . . . . . . . . . 29

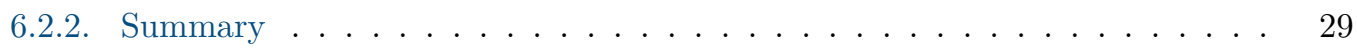


$\begin{array}{lr}\text { 7. Fluorescent nuclear track detection } & 31\end{array}$

7.1. Sample description . . . . . . . . . . . . . . . . . . . . 31

7.2. Vacancy diffusion coefficient in diamond . . . . . . . . . . . . . . . . . . 31

7.2.1. Resolution . . . . . . . . . . . . . . . . . 32

7.2.2. Localization . . . . . . . . . . . . . . . . . . 33

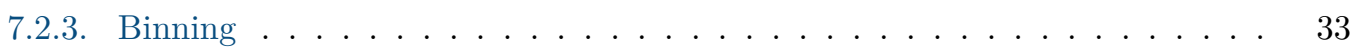



$\begin{array}{ll}\text { Quantum information processing } & 35\end{array}$

8. Geometric phase based quantum gates $\quad 37$

8.1. Intuitive example . . . . . . . . . . . . . . . . . . . . . . 37



8.3. Aharonov Anandan phase . . . . . . . . . . . . . . . . . . . . . 38

8.4. Abelian non-adiabatic geometric quantum gates . . . . . . . . . . . . . . . 39

8.5. Non-Abelian holonomies . . . . . . . . . . . . . . . . . . . . . . . . 39

8.6. Non-adiabatic non-Abelian holonomies . . . . . . . . . . . . . . . . . . 39

8.7. Intrinsic robustness . . . . . . . . . . . . . . . . . . . . . . 40

9. Performance evaluation $\quad 41$

9.1. Standard quantum process tomography . . . . . . . . . . . . . . . . 41

9.1.1. Quantum process matrix ................. 41

9.1.2. Evaluation routine . . . . . . . . . . . . . . . . . 41

9.1.3. Choice of basis operators . . . . . . . . . . . . . . . . 42

9.1.4. Experimental sequence . . . . . . . . . . . . . . 43

9.1.5. Rotating frame considerations . . . . . . . . . . . . . . 43

9.2. Randomized benchmarking . . . . . . . . . . . . . . . . . . . . . . . . . 44

9.2.1. Single qubit randomized benchmarking . . . . . . . . . . . . . 44

9.2.2. Experimental implementation . . . . . . . . . . . . . . 45

9.2.3. Fidelity and error scaling ................... . . 45

$\begin{array}{lr}\text { 10. Microwave control } & 47\end{array}$

10.1. Coherent spin manipulation . . . . . . . . . . . . . . . . . . 47

10.1.1. Sequence creation . . . . . . . . . . . . . . . . . 47

10.1.2. System frequency response . . . . . . . . . . . . . . . 48

$\begin{array}{ll}\text { 11. Superadiabatic geometric quantum gates } & \mathbf{5 1}\end{array}$

11.1. Superadiabatic geometric quantum driving . . . . . . . . . . . . . . . . . 51

11.1.1. Intuitive explanation . . . . . . . . . . . . . . . 51

11.1.2. Accelerated driving field frames . . . . . . . . . . . . . . . . 52

11.1.3. Superadiabatic Hamiltonian . . . . . . . . . . . . . . . . . . 53

11.2. Population transfer by a linearly chirped Gaussian pulse . . . . . . . . . . . . . . . 54

11.3. Non-commuting superadiabatic single-qubit quantum gates . . . . . . . . . . . 55

11.3.1. Pair of orthogonal cyclic states . . . . . . . . . . . . . 56

11.3.2. Proposed realization . . . . . . . . . . . . . . . 56

11.4. Parameter optimization . . . . . . . . . . . . . . . . . . 58 
11.5. Bloch-sphere trajectory _. . . . . . . . . . . . . . . . . . 59

11.6. Generalized SAGQG with arbitrary rotation angle . . . . . . . . . . . . . 60

11.7. Quantum gate fidelity . . . . . . . . . . . . . . . . . . . 61

11.7.1. Pauli gates . . . . . . . . . . . . . . . . . . 61

11.7.2. Hadamard gate . . . . . . . . . . . . . . . . . . 62

11.8. $\tau$ variation . . . . . . . . . . . . . . . . . . . . . . 62

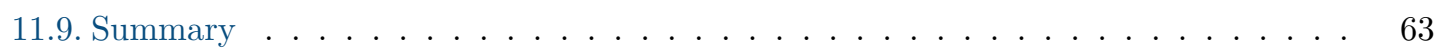

12. Benchmarking quantum gates $\quad 65$

12.1. Considered quantum gates modalities . . . . . . . . . . . . . . . 65

12.1.1. Dynamic quantum gates . . . . . . . . . . . . . . . 65

12.1.2. Abelian geometric quantum gate . . . . . . . . . . . . . . . 65

12.1.3. Non-Abelian non-adiabatic quantum gates . . . . . . . . . . . . 66

12.2. Boundary conditions . . . . . . . . . . . . . . . . . . . 67

12.3. Gate fidelity comparison . . . . . . . . . . . . . . . . . 67

12.4. Numerical robustness analysis . . . . . . . . . . . . . . . . . . 68

12.4.1. Implementation . . . . . . . . . . . . . . . . . . . . 68

12.4.2. Detuning definition . . . . . . . . . . . . . . . . . . 69

12.4.3. Results . . . . . . . . . . . . . . . . . 70

12.5. Experimental detuning analysis . . . . . . . . . . . . . . . . . . . . . 72

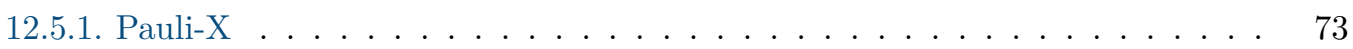



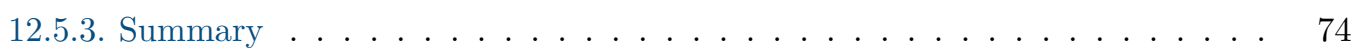

12.6. Average probability of error per gate . . . . . . . . . . . . . . . . . . 74

12.6.1. Modality comparison . . . . . . . . . . . . . . . . 74

12.6.2. Gate duration . . . . . . . . . . . . . . . . . . . . 75

12.6.3. Combining modalities . . . . . . . . . . . . . . . . 75

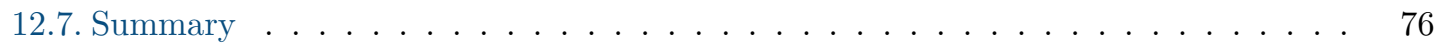

$\begin{array}{lr}\text { Discussion } & 79\end{array}$

$\begin{array}{lr}\text { 13. Conclusion and outlook } & \mathbf{8 1}\end{array}$

13.1. Super-resolution microscopy of solid-state spins . . . . . . . . . . . . . . . 81

13.1.1. ST1 center imaging . . . . . . . . . . . . . . . 81

13.1.2. Fluorescent nuclear track detection . . . . . . . . . . . . . . 81

13.2. High-fidelity quantum computation . . . . . . . . . . . . . . . . . . 82

13.2.1. Superadiabatic geometric quantum gates . . . . . . . . . . . . . 82

13.2.2. Benchmarking analysis . . . . . . . . . . . . . . 83

13.3. Concluding remarks ... . . . . . . . . . . . . . . 86

$\begin{array}{lr}\text { Bibliography } & 89\end{array}$

$\begin{array}{lr}\text { A. Data acquisition } & 101\end{array}$

A.1. Electronic control circuit . . . . . . . . . . . . . . . . . . . . . . . . 101

A.2. Superscript data acquisition . . . . . . . . . . . . . . . . . . . . 102

A.3. Resonance frequency tracking . . . . . . . . . . . . . . . . . . . . . . 102 
$\begin{array}{ll}\text { B. Sample characterization } & 105\end{array}$

B.1. NV center coherence times . . . . . . . . . . . . . . . . . . . . . . . . 105

B.2. Nuclear spin polarization . . . . . . . . . . . . . . . . . . . . 106

B.3. Line width . . . . . . . . . . . . . . . . . . . . . . . 108

$\begin{array}{ll}\text { C. Auxiliary calculations } & 109\end{array}$

C.1. Derivation of the superadiabatic Hamiltonian . . . . . . . . . . . . . . . . . 109

C.2. Time evolution operator . . . . . . . . . . . . . . . . . . . . . 111

D. Tuning the SAGQG robustness 113

D.1. Robustness as a function of $\Omega_{0} \ldots \ldots \ldots \ldots$. . . . . . . . . . . 113

D.2. Robustness as a function of $\tau \ldots \ldots \ldots \ldots$. . . . . . . . . . . . . . 113

$\begin{array}{ll}\text { List of Figures } & 117\end{array}$

$\begin{array}{ll}\text { List of Tables } & 119\end{array}$

$\begin{array}{ll}\text { Acknowledgement } & 121\end{array}$

$\begin{array}{ll}\text { Curriculum Vitae } & 123\end{array}$ 


\section{List of abbreviation}

\begin{tabular}{|c|c|}
\hline AA & Aharonov-Anandan \\
\hline AGQG & Abelian geometric quantum gate \\
\hline $\mathrm{APE}$ & average probability of error \\
\hline AWG & arbitrary waveform generator \\
\hline CVD & continuous vapour deposition \\
\hline DCM & dichroic mirror \\
\hline $\mathrm{DC}$ & direct current \\
\hline DYN & dynamic quantum gate \\
\hline ESR & electron spin resonance \\
\hline FNTD & fluorescent nuclear track detector \\
\hline FoR & frame of reference \\
\hline GR1 & "general radiation1" \\
\hline HPHT & high pressure, high temperature \\
\hline HQG & non-adiabatic non-Abelian holonomic quantum gate \\
\hline LCGP & linearly chirped Gaussian pulse \\
\hline MCS & multichannel scalar \\
\hline NISQ & noisy intermediate scale quantum computer \\
\hline NV & nitrogen vacancy \\
\hline ODMR & optically detected magnetic resonance \\
\hline PNTD & plastic nuclear track detector \\
\hline PSF & point spread function \\
\hline QECC & quantum error correction code \\
\hline QG & quantum gate \\
\hline QIP & quantum information processing \\
\hline QPT & quantum process tomography \\
\hline QS & quantum sensing \\
\hline qubit & quantum bit \\
\hline $\mathrm{RB}$ & randomized benchmarking \\
\hline SAGQG & superadiabatic geometric quantum gate \\
\hline SSNTD & solid-state nuclear track detector \\
\hline ST1 & "Stuttgart 1" \\
\hline STED & stimulated emission depletion \\
\hline TQD & transitionless quantum driving \\
\hline ZPL & zero phonon line \\
\hline
\end{tabular}



Introduction 



\section{Quantum information processing}

The foundations of quantum information processing dates back to the 1980's. R. Feynman highlighted that, due to discretization, classical computers are insufficient in simulating physics [1]. Instead he suggested that a probabilistic computer based on the laws of quantum mechanics needs to be utilized to describe nature precisely. D. Deutsch was the first to extend the possible range of applications to certain numeric problems promising a significant (exponential) speed up with respect to classical computers [2]. Today, computational problems theoretically benefiting from quantum supremacy [3] include, e.g., the prime factorization (Shor) [4], the search in an unsorted database (Grover) [5] and combinatorial optimization problems (e.g., travelling salesman problem) [6]. While the first represents the backbone of modern cryptography the two later might become powerful tools for big data applications. However, the full potential of universal quantum computers is not yet revealed and is an active field of research.

Experimental advances in the coherent control of quantum systems like cold atoms, superconducting qubits and solid-state spins paved the way for nowadays quantum registers. Scalable quantum computation is not yet achieved but state-of-the-art quantum annealer provide in the order of $\mathcal{O}\left(10^{3}\right)$ physical quantum bits (qubits) [7], while circuit based computer approach in the range of $\mathcal{O}\left(10^{2}\right)$ physical qubits $[8,9]$. Typically, the noise acting on the system increases with the number of qubits. Hence, quantum register in the current era are sometimes referred to as noisy intermediate scale quantum computers (NISQs) [10]. Two main challenges need to be solved in order to realize universal quantum computation. First, a further scaling of the number of qubits is necessary in order to tackle relevant problems inaccessible for modern super computers. E.g., the prime factorization of a 430-bit (130 digit) number requires in the order $\mathcal{O}\left(10^{9}\right)$ gate operations and 2, 150 computational quantum bits. Protection against decoherence by quantum error correction codes (QECCs), might increase the number of required physical qubits to $\mathcal{O}\left(10^{6}\right)[11]$. Second, quantum gates with an error rate between $10^{-2}$ and $10^{-6}$ need to be reliably implemented for QECC to become efficient $[11,12]$.

\subsection{Quantum bit operations}

A classical bit can take either of the binary values 0 or 1 . In contrast a qubit may occur in any superposition of its to two computational states $|0\rangle$ and $|1\rangle$. The state of a qubit

$$
|\psi\rangle=\cos \theta / 2|1\rangle+e^{i \varphi} \sin \theta / 2|0\rangle
$$

is defined by two continuous variables $\theta$ and $\varphi$, representing a two-dimensional state space. The angles $\theta$ and $\varphi$ might be utilized to illustrate the quantum state as a unit vector on the Blochsphere (Fig. 1.1.a). The poles of the sphere represent the pure states $|0\rangle$ and $|1\rangle$, respectively. In spherical coordinates $\theta$ represents the polar angle and $\varphi$ the azimuth. Analogously to classical logic gates, quantum gates (QGs) are the building blocks of computation in circuit based quantum computation. Classical strategies compensating for errors in the gate realization are prohibited by 




b

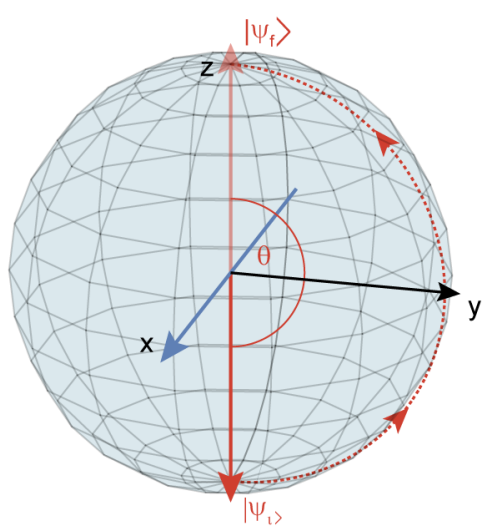

Figure 1.1.: Bloch sphere picture. (a) The state $|\psi\rangle$ of a qubit can be represented as a vector on the Bloch sphere defined by the two angles $\theta$ and $\varphi$. (b) The Pauli-X gate rotates the initial state $\left|\psi_{i}\right\rangle$ around the $\mathrm{x}$-axis (blue arrow) by an angle of $\pi$.

the quantum "no-cloning theorem" [13, 14].

One distinguishes quantum gates acting on single and multiple qubits. It can be shown that only two non-commuting single and one non-trivial two-qubit gates are needed to realize universal quantum computation (Sec. 1.1.3).

\subsubsection{Single qubit quantum gates}

While the control of a single classical bit only requires the logical NOT-operation, the control of a quantum bit is more demanding. QGs are described by an operation $U$ transferring the input state $\left|\psi_{i}\right\rangle$ of a qubit to an well-defined output state $\left|\psi_{f}\right\rangle=U\left|\psi_{i}\right\rangle$. $U$ can be visualized as a rotation of the state vector on the Bloch sphere by an angle $\theta$ around a well defined axis (Fig. 1.1.b). The operation $U$ is a unitary $2 \times 2$ transformation.

The Pauli matrices form a basis of the four-dimensional complex vector space of all complex $2 \times 2$ matrices [15]. Typically, they are denoted by $\sigma$ and are explicitly written as:

$$
\sigma_{x}=\left(\begin{array}{ll}
0 & 1 \\
1 & 0
\end{array}\right), \quad \sigma_{y}=\left(\begin{array}{cc}
0 & -i \\
i & 0
\end{array}\right), \quad \sigma_{z}=\left(\begin{array}{cc}
1 & 0 \\
0 & -1
\end{array}\right) .
$$

Exponentiating $i \sigma$ results in the special unitary group $\mathcal{S U}(2)$. Hence, any quantum operation can be represented by the Pauli matrices. Throughout the thesis, the generalized expression utilized to describe a rotation around $\hat{n}$ by an angle $\theta$ is

$$
R_{\hat{n}}(\theta)=\exp \left(-i \frac{\theta}{2} \hat{n} \cdot \vec{\sigma}\right)
$$

where $\vec{\sigma}=\left(\sigma_{x}, \sigma_{y}, \sigma_{z}\right)$ is the three component Pauli vector and $\hat{n}=\left(n_{x}, n_{y}, n_{z}\right)$ is a unit vector defining the axis of rotation. Rotations by $\theta=\pi$ around $\sigma_{x}, \sigma_{y}$ and $\sigma_{z}$ are referred to as Pauli $\mathrm{X}, \mathrm{Y}$ and $\mathrm{Z}$ gate, respectively. The identity operation leaves the input state $\left|\psi_{i}\right\rangle$ unchanged $(\theta=\{0,2 n \pi\}, n \in \mathbb{Z})$. Commuting gate operations are referred to as Abelian. Non-commuting (non-Abelian) gate operations are needed to perform arbitrary rotations of the spin state. 


\subsubsection{Multi qubit quantum gates}

Multi-qubit quantum gates acting on $k$ qubits are represented by a unitary matrix $U$ of dimension $2^{k} \times 2^{k}$. The corresponding quantum state is described by a $2^{k}$-dimensional complex vector. Mathematically the Bloch sphere representation can be extended to be $2^{k}$-dimensional, however the increased dimensionality prohibits its illustration. Note, in contrast to classical two-bit gates, featuring two inputs and one output, its quantum mechanical counterpart is reversible. Multi qubit gates typically feature one or more control qubits whose state decides if the operation is performed on the second qubit.

\subsubsection{Universal set of gates}

Intuitively, one might expect that a large number of quantum gates is needed to be able to perform arbitrary unitary transformation in a $2^{k}$-dimensional Hilbert space. However, it was shown that any unitary quantum operation can be efficiently approximated by a small universal set of gates with arbitrary precision [16]. It can be shown that every $2^{k}$-dimensional unitary matrix can be decomposed into second order tensors, such that a universal set consists of two non-commuting single qubit and one non-trivial two qubit gate [17, 18]. As a consequence, by identifying a small set of noise resilient quantum gates universal quantum computation on NISQs is within the realms of possibility.

\subsection{Scalable quantum computation}

The performance of sophisticated quantum algorithms requires in the order of $10^{6}$ physical qubits. As D. DiVincenzo stated in 2000, a quantum mechanical system needs to meet five (plus two) criteria in order to provide a realistic platform for for quantum computation:

I. A scalable physical system with well defined qubits

II. The ability to initialize the state of the qubits

III. Long decoherence times

IV. A "universal" set of quantum gates

V. A qubit-specific measurement capability

Scalability is required to tackle relevant problems inaccessible for classical computers. The initialization into a fiducial state makes it possible to start computing from a well-known input state. Full information on the final state can only be extracted by a precise measurement of each qubit individually. Long coherence times enable the application of a sufficient amount of quantum gate operation $\left(\mathcal{O}\left(10^{9}\right)\right)[11]$, while a universal set of gates is required to realize all possible quantum algorithms. Two additional criteria are required for the realization of quantum communication, a topic not covered within the scope of this thesis. A summary of today's most promising physical platforms for scalable quantum computation including, i.a., trapped atoms, superconductors and solid-state spins is given in [19]. 


\subsection{Quantum error correction codes}

In every quantum structure inevitable interactions with the environment cause loss of information due to decoherence. In contrast to classical computation architectures, the quantum "no cloning theorem" $[13,14]$ prohibits the duplication of quantum information and hence the use of classical correction protocols. QECCs, test if decoherence occured and restore the initial decoherence free quantum state by encoding a single computational quantum bit in several physical bits [20]. However, for these strategies to become efficacious the fidelity of the applied unitary operations need to exceed a certain threshold. Different works suggest that error thresholds per quantum gate between $10^{-6}$ and $10^{-2}$ are necessary $[11,12]$. Even though quantum gate fidelities close to one have been demonstrated utilizing dynamic phase based quantum gates (e.g., [21]) the realization of QECCs remains pending. Exploiting intrinsically robust, geometric phase based quantum gates (Ch. 8) might provide a powerful tool in the field of quantum information processing (QIP). 


\section{Nitrogen vacancy center in diamond}

Among the more than hundred known fluorescent defect centers in diamond [22] the nitrogen vacancy $(\mathrm{NV})$ center, consisting of a substitutional nitrogen atom and an adjacent vacant lattice site (Fig. 2.2.a), is the most studied one. First, a short note on the diamond host material is given. In Sec. 2.2 the NV centers unique optical and spin properties are discussed in detail, highlighting its potential for quantum information processing and quantum sensing applications. Finally, applications and current limitations of NV center are discussed (Sec. 2.3 and Sec. 2.4).

\subsection{Diamond}

Diamonds are semi conductors exhibiting a bandgap of $5.47 \mathrm{eV}$, equivalent to the energy of a photon in the ultra violet (UV) regime. As a consequence, a pure diamond lattice is transparent for visible light and colorless for the observer. In fact, color is originating from defects in the carbon lattice. The significant refractive index mismatch $\left(n_{\text {Diamond }}=2.4\right)$ between the diamond and standard immersion media represents a challenge for high-resolution imaging of color centers. At the same time, the thermal conductivity enables the application of remarkably high optical power, e.g., in stimulated emission depletion (STED) nanoscopy (Sec. 4.1.2). For research purposes typically industrial single or poly-crystalline diamonds are used. These are created by either the high-pressure-high-temperature (HPHT) or continuous vapour deposition (CVD) method [23]. Processing isotopically pure methane during the CVD growth generates a carbon lattice free of inherent ${ }^{13} \mathrm{C}$ nuclear spins [24] and results in a close to spin-noise free environment. The hardness in combination with advanced manufacturing techniques like reactive-ion-etching (RIE) and fast ionbeam (FIB) milling, enables the fabrication of nano-pillars [25], whispering gallery mode resonators [26], atomic force microscopy (AFM) tips [27] and solid-immersion lenses (SIL) [28], only to name a few. These advanced structures are utilized to increase the photon collection efficiency, position defect centers close to a specimen, store photons and increase the optical resolution. Hence, diamond is a versatile material providing a brought range of applications.

\subsection{Optical \& spin properties}

The nitrogen vacancy center in diamond occurs in at least two different charge states, the negatively charged $\mathrm{NV}^{-}$and the neutral $\mathrm{NV}^{0}$. Depending on the charge state the zero phonon line (ZPL) is located either at $637 \mathrm{~nm}\left(\mathrm{NV}^{-}\right)$or $575 \mathrm{~nm}\left(\mathrm{NV}^{0}\right)$ [29]. At room-temperature $(300 \mathrm{~K})$ the phonon sideband emission extends up to about $\sim 800 \mathrm{~nm}$ (Fig. 2.1.b). The excited states features a fluorescence lifetime of about $\tau_{\mathrm{NV}^{-}} \approx 12 \mathrm{~ns}$ and $\tau_{\mathrm{NV}^{0}} \approx 7 \mathrm{~ns}$ (Fig. 2.1.c) [30], respectively. Its photo-stability enables long-term measurements [31, 32]. Ionization and electron recaputering converts the NV center between its charge states under illumination. The conversion rate depends on the excitation intensity and wavelength [29]. It was shown that on illumination with green light $(\sim 532 \mathrm{~nm})$ of intensity $\sim 1 \mathrm{MW} \mathrm{cm}^{-2}$ the NV center is efficiently prepared in its negative charge state [33]. 
a



b





Figure 2.1.: Optical NV center properties. (a) Illustration of an nitrogen (orange) vacancy (red) center within the carbon (grey) diamond lattice. (b) Optical emission spectrum of an ensemble consisting of $\mathrm{NV}^{-}$and $\mathrm{NV}^{0}$ center excited at $532 \mathrm{~nm}$. Solid red lines indicated the ZPLs at $637 \mathrm{~nm}\left(\mathrm{NV}^{-}\right)$and $575 \mathrm{~nm}\left(\mathrm{NV}^{0}\right)$. (c) Averaged fluorescence lifetime measurement of an ensemble of $\mathrm{NV}^{0}$ (green) and $\mathrm{NV}^{-}$(blue) center, respectively. Dashed lines represent exponential fits extracting $\tau_{\mathrm{NV}^{-}} \approx 12 \mathrm{~ns}$ and $\tau_{\mathrm{NV}^{0}} \approx 7 \mathrm{~ns}$.

Here, the focus is on the $\mathrm{NV}^{-}$center, in the following referred to as $\mathrm{NV}$ center, which is utilized throughout the thesis because of its unique spin properties. The four electrons contributing to the NV center form a spin one system [34] (Fig. 2.2.b). The electron spin can be efficiently initialized into the $m_{s}=0$ by optical pumping, even at room-temperature [35]. A, for our purposes, dark decay channel via the singlet state (blue dashed arrows) only accessible for spins within $m_{s}= \pm 1$ ends in the ground state $m_{s}=0$ (Fig. 2.2.b). Therefore, after illumination with green light the $\mathrm{NV}$ center is initialized into the triplet ground-state $m_{s}=0$, providing a means for efficient optical electron spin polarization [36]. This is in stark contrast to the typically stochastic initialization based on the temperature dependent Boltzmann distribution in nuclear magnetic and electron paramagnetic resonance experiments. Due to its dark nature (no photon emission in the detection band) the same decay channel enables the readout of the electron spin state via state dependent fluorescence [37]. Figure 2.2.a presents the fluorescence level for spins initialized in $m_{s}=0$ (red) and $m_{s}= \pm 1$ (dark blue) when illuminated with green light starting at $t=0 \mathrm{~ns}$. The fluorescence difference (black) is measured with single photon counting devices in a detection window of length $t_{\mathrm{det}} \approx 300 \mathrm{~ns}$, allowing for optically detected magnetic resonance (ODMR) measurements [31].

The triplet ground state features a V-shaped level structure (Fig. 2.2.c) with a zero-field splitting of $D \approx 2.87 \mathrm{GHz}$ (e.g., [22, 34, 38]). The exact value of $D$ depends on the diamond sample, temperature, strain, electric and magnetic fields, making the NV center a powerful tool for quantum sensing applications. The full system Hamiltonian within an external magnetic field $B_{0}$ but no external driving is described by [22]:

$$
H=\underbrace{S D S}_{I}+\underbrace{g_{e} \beta_{e} B_{0} S}_{I I}+\underbrace{S A I}_{I I I}-\underbrace{g_{N} \beta_{N} I B}_{I V} .
$$

Here, term I and II describe the fine structure, term III the hyperfine interaction with the nitrogen nucleus and term IV the interaction with surrounding nuclear spins. The use of isotopically pure samples suppresses term IV. An external magnetic field lifts the degeneracy of the $| \pm\rangle$ state (term II). The hyperfine coupling of the electron spin to nitrogen nuclear spin increases the number of resonance to four $\left({ }^{14} \mathrm{~N}\right)$ and $\operatorname{six}\left({ }^{15} \mathrm{~N}\right)$, respectively. A magnetic field of $|B| \approx 400 \mathrm{G}$ aligned along the NV center axis tunes the triplet excited state to an avoided level crossing. Here, polarization of the nitrogen nuclear spin by electron-nuclear-spin flip-flops is enabled [39, 40] (Sec. B.2). In this way, the term III is eliminated and an effective three-level system with transition frequencies at 

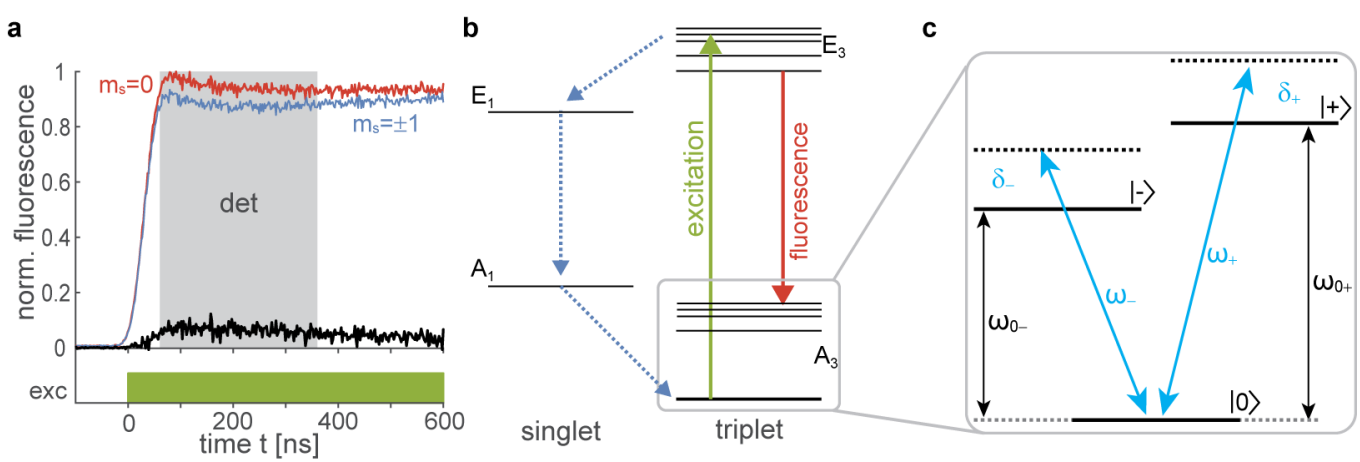

Figure 2.2.: NV center characteristics. (a) Spin state readout by state dependent fluorescence. The dark decay channel via the singlet state (blue in (b)) causes a decreased fluorescence for the $m_{s}= \pm 1$ state (blue) compared to the fluorescence of the $m_{s}=0$ state. (b) Jablonski diagram of the NV center. Green light excites the triplet state. Spontaneous emission is in the red due to the Stokes shift. (c) The V-shaped triplet ground state (grey rectangular in (b)) features transitions accessible by microwave driving fields (blue) and thereby allows for coherent spin state manipulation.

$\sim 1.73 \mathrm{GHz}(|0\rangle \leftrightarrow|-\rangle)$ and $\sim 4.01 \mathrm{GHz}(|0\rangle \leftrightarrow|+\rangle)$ is generated.

Both transitions $(|0\rangle \leftrightarrow| \pm\rangle)$ are accessible for microwave driving fields $\omega_{ \pm}$enabling coherent spin-manipulation [41] (Fig. 2.2.c). The interaction Hamiltonian for an NV center with polarized nuclear spin driven by microwave fields within the rotating frame is:

$$
H_{\text {int }}=\Delta_{+}|+\rangle\left\langle+\left|+\Delta_{-}\right|-\right\rangle\langle-|+\left(\Omega_{+}|0\rangle\left\langle+\left|+\Omega_{-}\right| 0\right\rangle\langle-|+\text { H.c. }\right) .
$$

Here, $\Delta_{ \pm}=\omega_{0 \pm}-\omega_{ \pm}$is the detuning of the driving field with angular frequency $\omega_{ \pm}$with respect to $\omega_{0 \pm}$, the energy difference between the states $|0\rangle \leftrightarrow| \pm\rangle . \Omega_{+}$and $\Omega_{-}$are the Rabi frequencies of the respective transition and H.c. the Hermitian conjugate. The large energy difference between the transitions $|0\rangle \leftrightarrow| \pm\rangle$ in combination with a polarized nitrogen nuclear spin makes it possible to treat the NV center as a simple two-level system when driving a single transition. The Hamiltonian simplifies to:

$$
H_{\text {int }}^{ \pm}=\Delta_{ \pm}| \pm\rangle\langle \pm|+\left(\Omega_{ \pm}|0\rangle\langle \pm|+\text { h.c. }\right) .
$$

Hence, depending on the experiment the NV center can be treated either as qubit or qutrit system.

In addition, NV centers in optimized diamond samples feature long electron spin coherence times of up to $0.6 \mathrm{~s}$ at cryogenic and several milli-seconds at room-temperature [42]. In conclusion, the properties of the NV center meet the II.-V. DiVincenzo criteria (Sec. 1.2) for quantum computation by default. Even though, the deterministic creation of scalable quantum architectures has not been achieved yet, experimental efforts accomplished first milestones (Sec. 2.4). Hence, the NV center potentially satisfies all of the DiVincenzo criteria (Sec. 1.1.3), providing a platform for scalable, universal quantum computation.

\subsection{Applications}

Due to its remarkable properties the NV center enriched various fields of research in the last decade. These include super-resolution microscopy, quantum sensing (QS) and quantum information processing (QIP). Similar properties are only featured by the recently discovered but still undetermined "Stuttgart 1" (ST1) center and the silicon vacancy (SiV) center. While the first lags 
protocols for the defect center generation, the second is restricted to applications at cryogenic temperatures.

The photo stability of the NV center drew attention in the field of super-resolution microscopy, where photo bleaching is one of the key challenges. Various techniques like stimulated emission depletion (STED) [32, 43], ground state depletion (GSDIM) [44, 45], spin-RESOLFT [46, 47], charge state depletion (CSD) [48] as well as stochastic optical reconstruction (STORM) [49] microscopy have been demonstrated in single crystalline bulk diamond. In nano crystals so far only STED [50] and STORM [51] imaging have been demonstrated. The availability of nano-diamonds containing NV centers as potentially non-toxic photostable markers for specimen in life science is currently limited by the lag of efficient functionalization protocols.

Additionally, the NV center is utilized as room-temperature quantum probe for quantum sensing (QS) applications. High precision quantum sensing of magnetic fields [52, 53, 54], electric fields $[55]$ and temperature $[56,57]$ have been demonstrated. Recently, the triggered detection of singleneuron action potentials has been reported [58]. The variety of measurable physical quantities make the NV center a potentially powerful tool for material science, life science and medicine.

In the field of quantum information processing (QIP) the NV center represents a qubit/qutrit platform under ambient conditions, avoiding the need for cryogenic or laser cooling systems. Single qubit operations by means of coherent microwave fields have been performed for dynamic [59] and geometric quantum gate modalities [60,61]. Multi-qubit gates have been realized by utilizing the backaction of the electron spins on a neighboring ${ }^{13} \mathrm{C}$ nuclear spin $[62,63,60]$ as well as the host nitrogen nuclear spin [64]. Additionally, by exploiting the coherent coupling by dipoledipole interaction of two NV center electron spins separated by roughly $\sim 10 \mathrm{~nm}$ the feasibility of electron spin based quantum registers has been proven [65]. STED microscopy enables the targeted initialization and readout of dipole-dipole coupled multi qubit quantum registers with sub-diffraction limited resolution [66]. While the spin-RESOLFT technique provides a means to readout out the NV center electron spin, the targeted initialization is prohibited [46]. The proposal avoiding the need for super-resolution readout by the utilization of a 'dark' spin chain bus sets significantly higher requirements to the sample generation [67], which already represents the bottleneck on the way to scalable quantum architectures.

\subsection{Current limitations in QIP}

Currently, the deterministic high spatial resolution generation of NV center as prerequisite for solid-state spin based quantum register is not yet achieved. However, tremendous experimental efforts are taken to overcome the limitations which are of solely technical nature. Small quantum register have been realized by the implantation of molecular [68] and atomic nitrogen [69] through nano-apertures. These approaches are highly stochastic and therefore do not provide a means for efficient scaling. The deterministic implantation of nitrogen ions by a modified Paul trap [70, 71] as well as by mirror charge detection [72] have been reported. Positioning of NV center by implantation through a pierced atomic force microscopy tip obtained $\sim 20 \mathrm{~nm}$ spatial resolution [73] but is not deterministic.

Since the NV center exhibits two constituents the sample needs to be annealed after implantation. In this way, vacancies are transport to the implanted nitrogen by diffusion. Additionally, annealing provides a means to cure damages of the carbon lattice originating from the nitrogen implantation. Next to elaborate techniques, like the implantation through boron doped layers [74], an improved understanding of the fundamental vacancy diffusion process in diamond might improve the NV 
center yield after annealing [75]. 



\section{Scope of this thesis}

The NV center in diamond, a quantum system under ambient conditions, is a promising platform for quantum information processing (QIP). However, the deterministic generation of defect centers with high spatial precision remains a challenging task. Besides difficulties in the implantation process of the nitrogen atom [70, 73], the NV center is suffering from a limited conversion efficiency [74]. An improved understanding of the underlying vacancy diffusion process might pave the way for improved annealing routines and thereby to reliable scaling. Here, it is demonstrated that STED nanoscopy offers the potential to determine the vacancy diffusion coefficient with high precision [75] (Ch. 7).

Due to the current technical limitations in the generation of NV center based quantum registers, a systematic screening for optically active defect centers featuring optically detected magnetic resonance (ODMR) is an active area of research [76]. When aiming to realize quantum registers by exploiting the electron spin dipole-dipole interaction the diffraction unlimited, targeted, read-out of the spin state is compulsory [65]. As a consequence, the demonstration of super-resolution microscopy represents an important step in the screening procedure. Towards this end, STED imaging of the recently discovered but still unidentified ST1 center in diamond [77] is demonstrated (Ch. 6).

No matter, which physical platform will in the end facilitate a scalable quantum architecture, it needs to provide a universal set of quantum operations with close to perfect fidelity [12]. Geometric phase based quantum gates represent a promising resource for QIP, due to their intrinsic resilience against local noise. Originally derived in the adiabatic limit [78], first experiments realized nonadiabatic universal sets of single-qubit geometric quantum gates (e.g, [61, 79, 80]). In this way, the application in realistic, decoherence limited quantum systems was enabled. Here, a recently proposed set of non-Abelian, single qubit, superadiabatic geometric quantum gates (SAGQGs) is realized [81] (Ch. 11). The SAGQG, realized on a two-level system employing a single driving field, offers the potential for high-fidelity quantum computation, while keeping the experimental resources to its minimum.

SAGQGs enrich the selection of quantum gate modalities including dynamic, adiabatic geometric, non-adiabatic Abelian geometric and non-adiabatic holonomic approaches. This variety raises the question which of the versatile options is providing the highest resilience against noise. For a set of non-adiabatic gate modalities the robustness with respect to constant control parameter imperfections for a realistic set of experimental boundary conditions is investigated (Ch. 12). This benchmark analysis provides a powerful tool for the identification of the most robust, universal set of QGs for the considered physical platform (Ch. 13). 

Super-resolution microscopy 



\section{Theoretical background}

Here, the physical concept of the techniques used in Ch. 5-7 are presented. These include confocal and STED microscopy, as well as solid-state nuclear track detectors (SSNTDs).

\subsection{Fluorescence imaging of solid-state spins}

Fluorescence imaging and optical addressing represent main challenges in the observation and control of several quantum systems like ultra-cold atoms [82], trapped atomic ions [83], BoseEinstein-condensates [84] and solid-state spins [31]. Often, the relevant quantum information is encoded in the final position of the emitter, thereby raising the need for high spatial resolution widefield imaging. Towards this end, ultra-high vacuum compatible objective lenses are designed [85] and sophisticated algorithms located emitters with a precision below the diffraction limit of light (e.g., [86]).

In contrast, the relevant quantum information of the NV center electron spin is extracted by measuring the fluorescence level originating from a defect center at a well-defined position (Sec. 2.2). As a consequence, the advantages of the conventional scanning confocal microscopy (Sec. 4.1.1) can be exploited in quantum information processing (QIP) and quantum sensing (QS) applications based on solid-state spins. However, in order to guarantee a sufficient dipole-dipole coupling of the electron spins, a separation between defect centers of $\lesssim 30 \mathrm{~nm}$ [65] is needed in quantum registers. Therefore, super-resolution imaging techniques for the deterministic addressing and read-out of individual electron spins are required. Diffraction-unlimited imaging of single defect spins has been demonstrated utilizing different techniques (Sec. 2.3). Here, the focus is on STED microscopy (Sec. 4.1.2), the technique which was applied within this thesis. At the same time, so far only STED and spin-RESOLFT microscopy proved sub-diffraction limited spin-state readout of individual solid-state spins within small ensembles [66, 46].

\subsubsection{Confocal microscopy}

In confocal microscopy, only a small volume of diffraction-limited extend is illuminated at a time. Already in 1873, E. Abbe discovered that the radius of the focal spot has a lower limit given by [87]

$$
r_{\text {Abbe }}=\frac{\lambda}{2 n \sin \alpha}=\frac{\lambda}{2 \cdot \mathrm{NA}},
$$

where $\lambda$ is the wavelength of light, $n$ the refractive index of the immersion medium, $\alpha$ is half the opening angle of the objective lens and NA $=n \sin \alpha$ its numerical aperture. For light in the visible regime and high quality objective lenses the resolution is typically limited to $r_{\text {Abbe }} \gtrsim 200 \mathrm{~nm}$. Emitters located within the diffraction limited focal volume are excited into a higher energetic state by absorption of an incoming photon. Relaxation into the ground state emits a, with respect to the excitation light, red-shifted photon (Stokes shift). Separation of the excitation and fluorescence light is performed by a dichroic mirror (DCM). Focusing the collected light on a pinhole and 



Figure 4.1.: Confocal microscopy. (a) Schematic drawing of a confocal microscope. Excitation light (green) originating from a point-source is focused to a diffraction limited spot. Emitters within the focal spot get excited by absorbing an incoming photon. Red-shifted fluorescence is separated from the excitation beam by a dichroic mirror (DCM) and detected after passing a confocal pinhole. Light originating from outside the focal volume (orange) is blocked at the pinhole. (b) Calculated confocal image of a point-like emitter. (c) Slice through the center of the intensity distribution (white line in b). The Abbe-limit defines the distance $r_{\text {Abbe }}$ between the center of the distribution and the first intensity minimum.

measuring it with a sensitive photon counting device completes the microscope. As indicated in Fig. 4.1.a, background fluorescence originating from outside the focal volume (orange beam) is strongly suppressed by the pinhole such that an improved signal-to-background ratio is obtained. Commonly, images are acquired in a pixelated manner by scanning the sample or excitation beam over the region of interest. Confocal microscopy is especially suited for efficient initialization with moderate optical powers as focusing the excitation beam to a diffraction limited spot generates high intensities.

\subsubsection{STED microscopy}

Basically, a STED microscope [88] suppresses the fluorescence in a well-defined area by pumping the emitter from the excited state (on) into the ground state (off) by means of stimulated emission. Typically, the diffraction-limited confocal excitation beam is overlapped with a, in the focal plain of the objective lens, doughnut-shaped depletion beam featuring an intensity minimum at the position of the excitation maximum (Fig. 4.2.a-b). This beam depletes emitters into the ground state, where photon emission is prohibited, resulting in a reduced effective point spread function (PSF) and hence in breaking the diffraction barrier of light (c). The resolution of a STED microscope scales with the intensity $I$ of the depletion beam according to [89]

$$
r_{\mathrm{STED}}=\frac{\lambda}{2 n \sin \alpha \sqrt{1+\frac{I}{I_{\mathrm{S}}}}}=\frac{r_{\text {Abbe }}}{\sqrt{1+\frac{I}{I_{\mathrm{S}}}}},
$$

where $I_{\mathrm{S}}$ is the saturation intensity of the emitter. From Eq. 4.2 one can immediately conduct that the achievable spatial resolution is theoretically unlimited. However, technical limitations like the available laser power, sample photo damage, the size of the fluorophore and the quality of the doughnut's intensity minimum restrict the resolution to typically $r \sim 20 \mathrm{~nm}$ in biological specimen [90]. By today, the highest obtained (one-dimensional) optical resolution demonstrated on a STED microscope is $r \sim 2.4 \mathrm{~nm}$ utilizing the solid-state spin dedicated to the nitrogen vacancy 
a
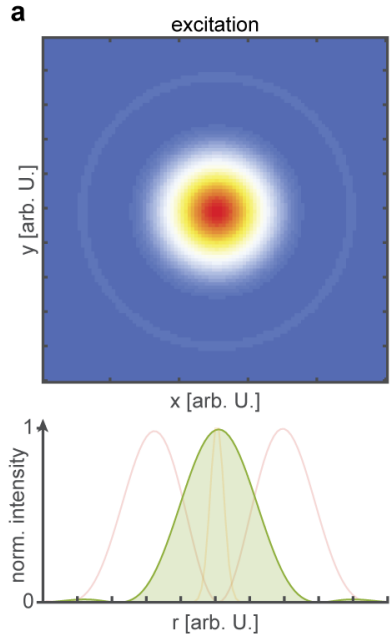

b

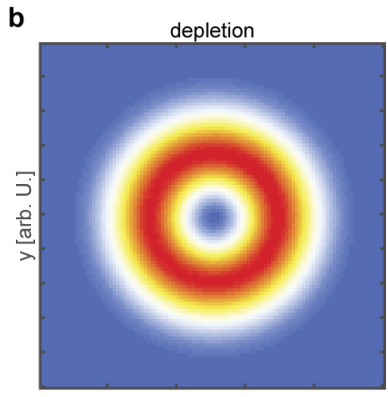

$\mathrm{x}$ [arb. U.]



r [arb. U.]
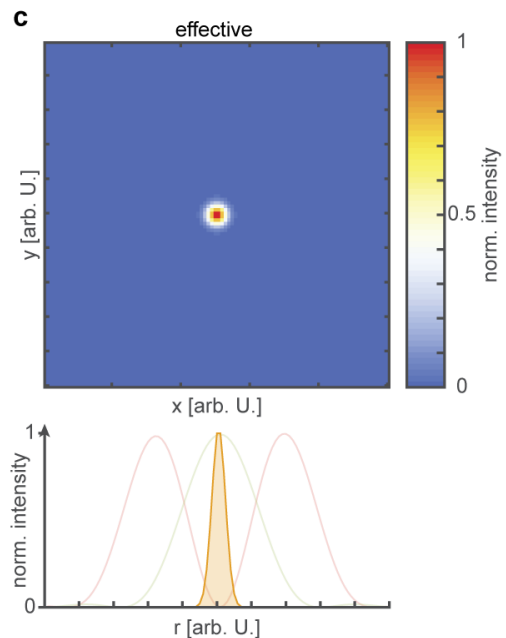

Figure 4.2.: Illustration of the STED microscopy principle. (a) Diffraction limited point spread function of the excitation beam. (b) Doughnut shape stimulated emission depletion beam featuring an intensity zero at its center position. (c) The effective point-spread-function of the system exhibits a resolution beyond the diffraction limit. Lower plots represent a cut through the center of the respective intensity distribution.

center in a diamond sample possessing a nano-fabricated solid-immersion lens [43]. It has been shown that STED microscopy enables the targeted readout of solid-state spins with sub-diffraction limited resolution while preserving the spin state population of defect centers outside the volume of the effective PSF [66]. This unique property makes STED microscopy the most promising super-resolution imaging technique for QIP and QS.

\subsection{Solid-state nuclear track detection}

Solid-state nuclear track detectors allow to investigate the trace of single ionizing particles by visualizing the damage they inflicted to a solid. For decades, plastic nuclear track detectors (PNTDs) represented the state-of-the-art, but were limited in spatial resolution and the ability to resolve damages induced to surfaces. Aluminium-oxide-based fluorescent nuclear track detectors (FNTDs) have been developed utilizing fluorescent defects in crystals to visualize ion trajectories by highresolution optical imaging [91]. Fluorescence microscopy in transparent solids enabled the threedimensional imaging of whole ionizing radiation traces. Recently, the variety of FNTD has been enriched by the NV center in diamond [92]. Here, a comparative overview on the physical processes of origin of the PNTDs as well as the aluminium oxide and diamond based FNTD is given, highlighting the advantages and potentials of FNTDs compared to PNTDs.

\subsubsection{Plastic nuclear track detectors}

Plastic nuclear track detectors utilize polymers (e.g., CR-39), which form radicals under ionizing radiation. After irradiation, the radicals form a latent ion trace within the sample [93]. Radicals and hence locations traversed by the radiation are revealed by surface etching of the sample. These regions etch faster than the surrounding material, resulting in a topographic radiation map. Subsequent analysis by atomic force [94] or conventional optical microscopy [95] extracts the number and position of the ions. The typically achievable resolution $(\sim 1 \mu \mathrm{m})$ makes PNTDs a powerful and cheep platform for dosimetry. However, the required etching process limits the 
analysis to surfaces. Three-dimensional imaging of whole ion traces remains challenging.

\subsubsection{Fluorescent nuclear track detectors}

The optical imaging of nuclear tracks utilizing magnesium and carbon doped aluminium oxide single crystals $\left(\mathrm{Al}_{2} \mathrm{O}_{3}: \mathrm{C}, \mathrm{Mg}\right)$, referred to as FNTDs, enriched the palette of SSNTDs. In this system, ionization by high energetic ions generates electrons along the ion track, transforming $\mathrm{F}^{2+}(2 \mathrm{Mg})$ into the fluorescent $\mathrm{F}^{+}(2 \mathrm{Mg})$ defect by electron capturing [96]. In contrast to PNTDs, the traces in aluminium-oxide-based FNTDs are observable without any additional treatment using fluorescence microscopy. Standard confocal scanning microscopy demonstrated spatial correlation of ion trajectories and cell damage providing a potentially powerful tool in radiation therapy [97]. As shown in Sec. 4.1.1 the spatial resolution of the traces obtained in confocal microscopyis limited by diffraction. Also with STED microscopy, resolutions below $\sim 100 \mathrm{~nm}$ could not be achieved in aluminium-oxide-based FNTD imaging.

In a similar manner, NV centers in diamond can be utilized as FNTD [98]. However, the defect center creation is based on a different physical process. By irradiating a nitrogen-containing diamond sample with heavy ions of high energy (hundreds of $\mathrm{MeV}$ ), vacancies are created along the ions trajectory. Vacancy diffuse during the subsequent annealing generates NV centers when a vacancy is caught by an intrinsic nitrogen atom. Similar to the PNTD, the exact characteristics of the NV center based FNTD depend on the processing of the sample as well as its nitrogen content [75]. The analysis of NV center based FNTDs offers the potential to determine physical constants like the vacancy diffusion coefficient in diamond (see Ch. 7 and [75]). Additionally, the colocalization of cell damage and ion trajcetories potentially enabels new insights in medical radiation treatments [96]. 


\section{A STED microscope for imaging of solid-state spins}

The demands a microscope for the coherent manipulation of optically detected solid-state spins needs to meet are numerous and technically challenging. For example: High mechanical stability, precise timing of optical and microwave driving fields as well as a high degree of automation are key requirements to perform long-term measurements within a spot smaller than the diffraction-limit. Here, the experimental apparatus designed and built for sample-scanning super-resolution STED imaging of various point defects in diamond (Ch. 6 and Ch. 7) is described and characterized. Additional components required for the coherent control of the NV center electron spin are discussed in Ch. 10.

\subsection{Optical configuration}

In essence, the microscope is build as a standard confocal microscope with the ability to perform super-resolution microscopy by means of an additional STED laser beam. A simplified sketch of the optical components of the system is depicted in Fig. 5.1. Confocal measurements (except for fluorescence lifetime imaging) are performed by continuous wave excitation at $546 \mathrm{~nm}$ wavelength (2RU-VFL-P-1500-546-R, MPB Communications Inc.) with an optical power of up to 1 W. For time-gated STED measurements pulsed laser excitation is provided by a laserdiode (PicoQuant LDH-P-FA-530XL) at a wavelength of $532 \mathrm{~nm}$ with an optical power of $\leq 100 \mathrm{~mW}$ and a pulse length of $100 \mathrm{ps}$. The trigger synchronising depletion and excitation laser is provided by the STED laser at a repetition rate of $20-30 \mathrm{MHz}$ (Sec. A.1). The optical power of each excitation beams is modulated and switched by a separate acousto-optical modulator (AOM) (AA.MT250-A0.2-VIS, AA Optoelectronics) in double-pass configuration. In this way an extinction ratio of $\geq 66 \mathrm{~dB}$ is guaranteed. This necessary to prevent interaction of the light with the measured qubit during the realization of coherent microwave experiments. Both excitation beams are superimposed and coupled into the same optical single-mode fibre. Transmission through the optical fiber cleans the mode profile and enables fast switching between continuous wave and pulsed excitation. A dichroic mirror (ZT594rdc, Chroma) guides the excitation beams into the objective lens (HCX PL APO 100x/1.4-0.7, Leica) which focuses them to a diffraction limited spot. The designed Abbe radius of the excitation spot is $r=\lambda_{\text {exc }} /(2 \mathrm{NA}) \approx 190 \mathrm{~nm}$ [87]. An active objective temperature controller (3508, Eurotherm) stabilizes the temperature to $30^{\circ} \mathrm{C}$ at the sample and pre-compensates heating effects induced by the near-infrared STED beam. Depolarization effects [99] are minimized, by tuning the polarization to be circular in the back aperture of the objective lens, utilizing retardation plates (B. Halle). Redshifted fluorescence originating from the excitation volume is collected by the objective lens, passes through the dichroic mirror and is focused onto the confocal pinhole. Two avalanche photodiodes (APDs) (SPCM-AQRH-13, Excelitas) forming a Hanbury Brown-Twiss interferometer [100] detect the fluorescence. This configuration additionally enables the determination of the number of emitters within the focal volume by measuring the second 


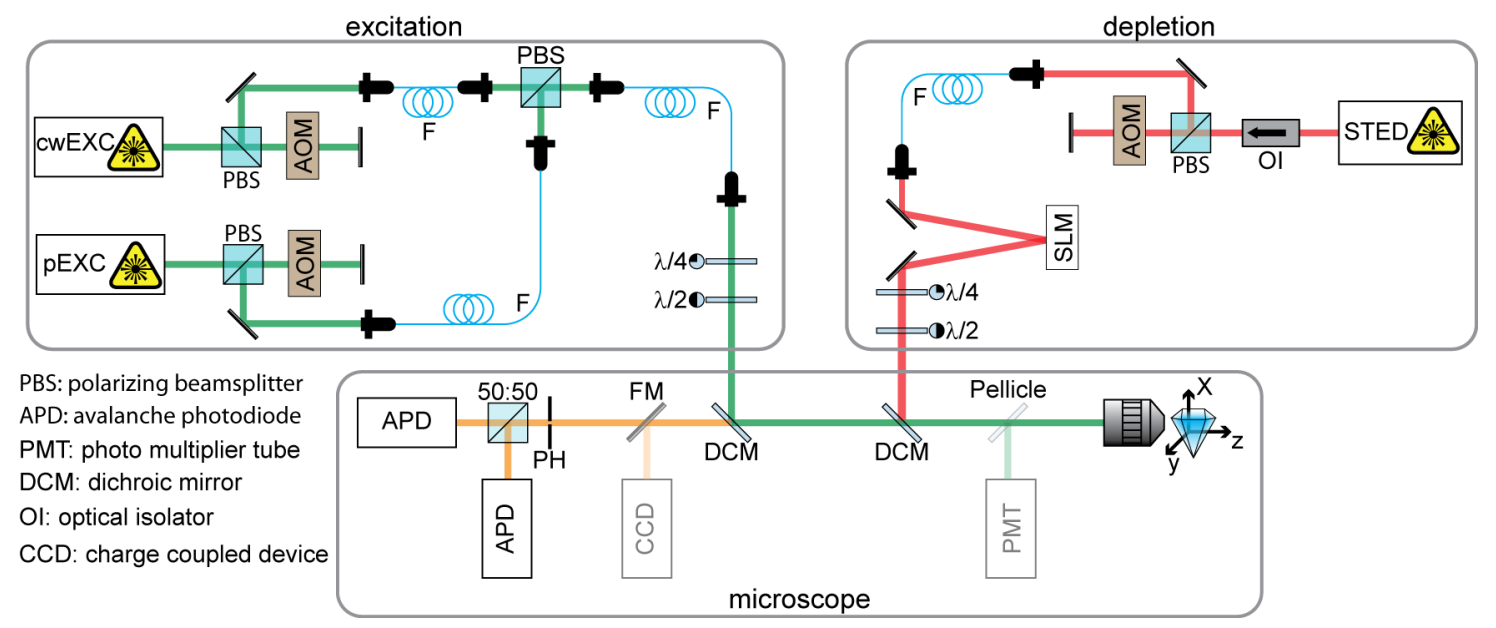

Figure 5.1.: Simplified sketch of the experimental apparatus. In general the apparatus can be divided into three parts. The preparation and superimposing of two excitation beams (green), the preparation and doughnut generation by means of a SLM of the stimulated emission depletion beam (red) and the microscope guiding the beams into the objective lens as well as collecting the emitted fluorescence (orange) with two APDs in a Hanbury Brown and Twiss configuration. The STED laser is protected from damages by back reflections by an optical isolator (OI). AOMs in double-pass configuration allow to regulate the optical power and switch between the beams. Superimposing and separation of different wavelengths is performed by DCMs. Optical fibers (F) are utilized to overlap and transfer beams. Waves plates $(\lambda / 2$ and $\lambda / 4)$ allow for a precise control of the polarization within the focal plain. A flip mirror (FM) in combination with a charge-coupled device (CCD) enables wide-field imaging. By inserting a pellicle into the beam path,the back-reflection of gold-beads can be imaged onto a PMT allowing a precise measurement of the PSF.

order correlation function [101, 102]. Photon detection events are stored into a multiple-event time digitizer (MCS6A, Fast ComTech GmbH) exhibiting 100 ps time-resolution, enabling time-gated detection [103].

Super-resolution imaging is realized by illuminating the sample with a STED beam (Sec. 4.1.2). The STED laser provides up to $10 \mathrm{~W}$ optical power at $775 \mathrm{~nm}$ wavelength. Each optical pulse, provided at a repetition rate of $20-30 \mathrm{MHz}$, features a rectangular temporal envelope of two nanoseconds duration. An optical isolator placed immediately in front of the STED laser aperture protects the device from damages by back-reflection (Katana, OneFive). Again, modulation and switching of the STED beam is performed by an AOM (AA.MT200-0.5-800, AA Optoelectronics) in double-pass configuration. Currently, the maximally applicable STED power is limited to $600 \mathrm{~mW}$ by the damage threshold of the optical fiber used for cleaning the mode profile and guiding of the beam. In future, this limitation will be overcome by utilizing an optical hollow-core fiber suitable for the transmission of high optical powers. After transmission through the optical fiber, a spatial light modulator (SLM) (LCOS-SLM X13267-02, Hamamatsu) imprints a user-defined phase mask onto the beam. The phase mask is used to generate the doughnut in the focus of the objective lens and to control optical aberrations (Sec. 5.3). An additional dichroic mirror (F39-758, AHF Analysetechnik) superimposes the STED beam with the excitation beams and guides it into the objective lens. A detailed discussion on the obtained optical resolution for nitrogen vacancy (NV) and "Stuttgart 1" (ST1) center is presented in Sec. 6.2 and Sec. 7.2.1, respectively.

A magnetically mounted pellicle beam splitter (BP145B1, Thorlabs) can be introduced into the beam path to analyse the excitation and depletion beam PSFs independently of the confocal pinhole. It guides the reflective signal of beads onto a photomultiplier tube (PMT) (H10723, Hamamatsu). By sample scanning microscopy the full information on the PSFs can be extracted. 

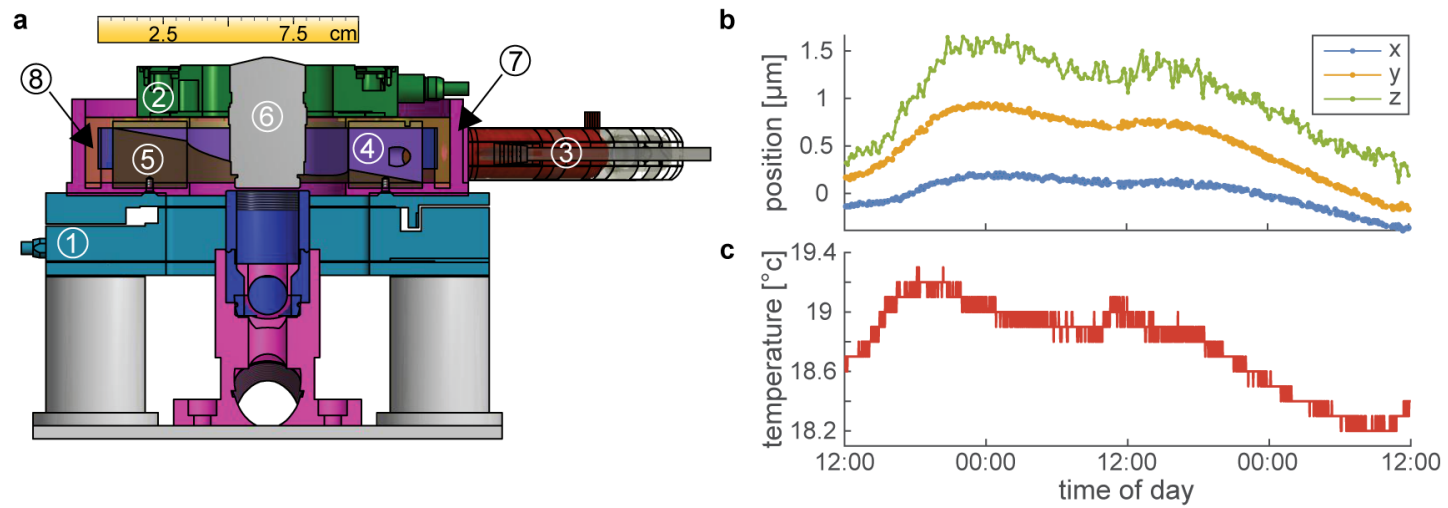

Figure 5.2.: The scanning stage system. (a) Technical drawing of the stage system, consisting of (1) coarse- and (2) fine-stage, (5) fixed and (4) moving wedge, the (7) guiding hollow cylinder and the 8 translating cylinder. (b) Long-term tracking position in $\mathrm{x}, \mathrm{y}$ and $\mathrm{z}$-direction of a single emitter as measure of the mechanical stability. (c) Temperature measured during the same period outside the isolation box of the objective lens.

The imaging of a large area of the sample on a charge-coupled device (Phillips) is enabled a magnetically mounted wide-field lens in the excitation path in combination with a motorized flip mirror in the detection. The wide-field configuration is used for a simplified navigation on the sample.

\subsection{Scanning stage system}

The demands on the sample scanning system are particularly versatile. On the one hand, a scanning range of several millimeters in all spatial dimensions is needed to manoeuvre precisely on the diamond sample. On the other, a position accuracy below one nanometre is required to reliably perform high spatial resolution imaging. At the same time, the system needs to be longterm stable to perform spin manipulation experiments at single quantum emitters located within the effective PSF. To our best knowledge, no commercially available system is satisfying these demands. Therefore, in close collaboration with our optical engineer and mechanical workshop a mechanically stable, long-range scanning system was designed and built in-house (Fig. 5.2.a).

A design concatenating a coarse and fine scanning stage was chosen. Coarse xy-positioning is performed by a piezo stage (M-686.D64, Physik Instrumente, turquoise) with a travel range of $25 \mathrm{~mm} \times 25 \mathrm{~mm}$. This stage features a self-locking system, retaining the sample position after reaching the region of interest. The fine positioning and scanning is performed by an additional stage (P-562.3CD, Physik Instrumente, green) featuring a travel range of $200 \mu \mathrm{m} \times 200 \mu \mathrm{m} \times 200 \mu \mathrm{m}$ with a closed loop resolution of $0.2 \mathrm{~nm}$.

Commercial stages with a long travel range in z-direction are usually heavy and bulky. To avoid an over or asymmetric load of the xy-coarse stage, an opto-mechanical component was designed to translate the movement of a linear actuator (M-230.25, Physik Instrumente) into a z-movement of the sample (Fig. 5.2.a). The linear actuator pushes a sliding wedge (purple) onto a wedge fixed to the coarse xy-stage (brown), thereby lifting a cylinder (yellow) which is eventually holding the fine stage and sample. To fix the position of the cylinder in xy-direction it is inserted into a hollow cylinder (pink) produced with small mechanical tolerances $(\mathcal{O}(5 \mu \mathrm{m}))$. A rotation of the cylinders with respect to each other is prevented by a tongue and groove joint. Eight springs provide a counterforce keeping the sliding cylinder in contact with the linear actuator. Hence, the position 
of linear actuator defines the sample height at all times. The wedge angle of $14^{\circ}$ converts the horizontal movement of the linear actuator into the z-movement of the sample by a translation factor of $\times 0.25$. This results in a $\mathrm{z}$ travel range of $4.5 \mathrm{~mm}$. A position detector (MHR 100, Althen) measuring the vertical distance between the two cylinders provides an additional feedback on the position.

In most super-resolution imaging applications only the relative position of the emitters is of interest, while the absolute position does not contain any additional information. In contrast, for the optical addressing and readout of solid-state spins the emitters need to be precisely located within the excitation volume. The designed control software strictly synchronises the detection with the position signal of the scanning stage to ensure that imaging is performed within the absolute frame of the fine-stage. This is not necessarily given in other imaging routines. Images are acquired in line-scanning mode. Towards this end waveforms are generated which define the stage position at given times. The waveforms consist of three phases: acceleration, linear movement and deceleration. Data are acquired during the linear movement, where the constant velocity of the stage relates dwell time and pixel size, such that the dwell time defines the pixel size. Depending on the loaded sample and chosen scan parameters the acceleration needs to be limited (softwarebased).

\section{Long term stability}

The stability of the stage system is tested by continuously taking xy and xz-confocal images of a single emitter. Subsequently, a two dimensional Gaussian distribution is fitted to the acquired images, extracting the current position of the emitter. In Fig. 5.2.b the fitted x, y and z-position are plotted versus the experiment duration of $48 \mathrm{~h}$. The position data shows a strong correlation to the ambient temperature (Fig. 5.2.c). Thermal effects are more severe than the settling behaviour of the concatenated stage system. However, within the typical retracking intervals of five to ten minutes, the observed drifts are much smaller than the confocal resolution $(\mathcal{O}(20 \mathrm{~nm}))$. Therefore, the stability of the system is sufficient for single-qubit experiments. For coherent spin manipulation experiments with high STED resolution an active sample stabilization based on a reflective infrared signal or the transmitted excitation light should be considered [104, 105]. A first study proving the feasability of an active stabilization system has been performed in [106].

\section{Large field scanning}

The designed stage system in combination with the custom-written control software allows for the screening of complete diamond samples in an automatized manner. To do so, an xy image $(150 \mu \mathrm{m} \times 150 \mu \mathrm{m})$ is acquired using the fine stage, subsequently the coarse stage is repositioned and the next image is taken. In post-processing all sub-images are concatenated according to the position of the coarse stage. Whole diamond samples with edge length of several millimeters can be imaged with high-resolution $(1 \mu \mathrm{m})$ during an acquisition time of one day. The resulting images are a valuable tool for sample pre-characterization. Aforementioned active z-position stabilization can be implemented to improve the image quality further by keeping the depth of interest within the focal plain and thereby compensating for the unevenness and tilt of the sample. 


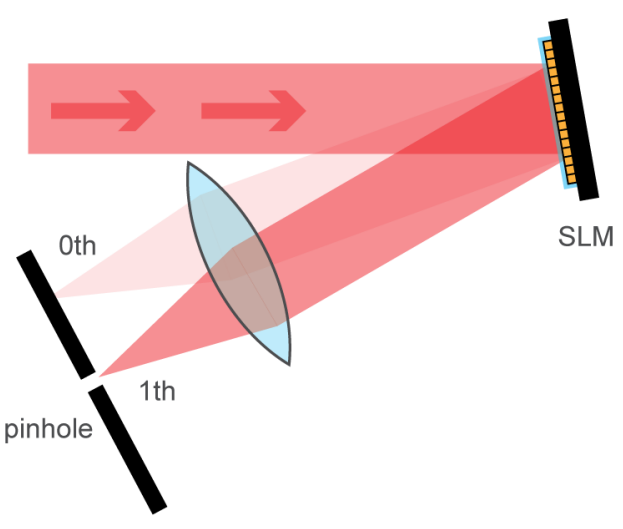

b

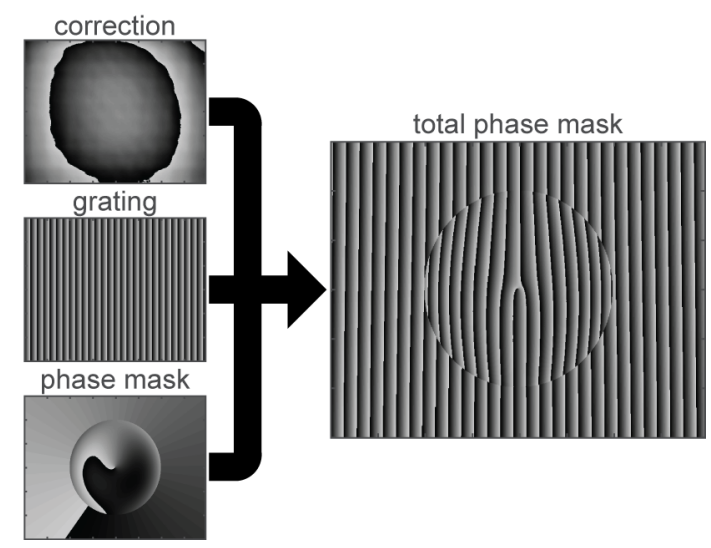

Figure 5.3.: Illustration of the SLM configuration. (a) An incident beam is reflected at the SLM chip, where the phase of the beam is modulated. Due to the applied optical grating the reflected beam is diffracted into• of several orders. Focusing onto a pinhole ensures propagation of the desired beam order to the sample, only. (b) Illustration of the three components contributing to the total phase mask. Surface correction data is provided by the distributor while the diffraction grating is designed according to the experimental needs. The shown phase mask consists of a vortex and a vertical coma of $0.4 \lambda$ amplitude. Summation of these three contributions results in the total phase mask as shown on the SLM.

\subsection{Spatial light modulation}

In recent years, the improved quality of SLMs boosted the use of active optical elements in optical microscopy [107]. Precise phase handling enables the compensation of system inherent aberrations and corrections depending on the imaging depth within the specimen . A reduction of optical aberrations leads in STED/RESOLFT microscopy to an improved quality of the doughnut and an increased resolution.

Additionally, SLMs allow for the alignment of the phase mask with respect to the beam without mechanical motion. Therefore, no settling behaviour of screws distorts the system after alignment. Similarly, the overlap of excitation and STED beam can be fine-adjusted fully electronically by adding tips and tilts to the phase mask.

\subsubsection{Experimental implementation}

The SLM utilized for the phase vortex creation and aberration control of the STED beam is used in reflective mode. In order to guarantee optimal performance the incident angle of the beam should be less than ten degree. Due to its pixelation, the SLM chip itself acts as an optical grating generating higher order diffraction beams. Commonly, a well controlled optical grating is added to the modulation pattern to prevent distortions of the beam with its higher orders. Separation of the different diffraction orders is accomplished by focusing the reflected signal onto a pinhole (Fig. 5.3.a).

A custom-written MATLAB program generates the phase mask as an 8-bit grey-scale image. Subsequently, the image is send to a computer that provides the phase mask to the SLM controller via a digital visual interface (DVI) connection. Creation of the phase mask is performed in three steps (Fig. 5.3.b). First, a wavelength dependent calibration mask (provided by the supplier) is loaded which compensates for the surface unevenness in the active area, ideally leading to a planar wavefront. Second, the diffraction grating is added. The periodicity of the grating was set to 16 pixels resulting in a diffraction efficiency in the first order of about $70 \%$ and a beam separation 
angle between 0 th and 1st order of roughly $3.5^{\circ}$. Each phase mask contains at least these two components in order to guide the beam unperturbed to the objective lens. In a final step, the beam manipulation phase mask is calculated. At the current state, the software allows for adding a phase-spiral or step needed for the creation of 2D and 1D doughnuts, respectively. Additionally, the possibility to manually compensate for optical aberrations by adding Zernike polynomials up to radial order four of arbitrary beam diameter is implemented. By summation of the three single images the total phase mask as presented on the SLM is generated. Automatized aberration control routines could be implemented in future (e.g. [108]). 


\section{STED imaging of ST1 centers in diamond}

Due to the technical difficulties in the deterministic high spatial resolution generation of NV centers, a systematic screening aiming to identify unknown defect center with similar properties is currently performed [76]. However, the coupling via the electron dipole-dipole interaction fundamentally requires a defect center separation in the order of $\leq 30 \mathrm{~nm}$ in quantum information processing (QIP) architectures [65]. Consequentially, the targeted spin state readout needs to be performed with an optical resolution unlimited by diffraction. STED microscopy, capable of coordinate targeted spin-state readout [66], is integrated as part of the screening procedure.

Recently, an up to the present unidentified fluorescent defect in diamond, referred to as "Stuttgart 1" (ST1) center, has been discovered [77, 109]. Next to the NV center it is the only defect center known to feature optically detected magnetic resonance (ODMR) at room temperature, making it an additional promising candidate for solid-state spin based QIP and quantum sensing (QS) applications (Sec. 6.1). Here, first STED microscopy imaging of the ST1 defect is demonstrated, paving the way for super-resolution applications (Sec. 6.2). In Sec. 6.2.1 the resolution scaling of ST1 and NV centers as a function of the STED intensity is compared, utilizing the same experimental apparatus.

\subsection{ST1 center properties}

In comparison to the NV center the ST1 center emission spectrum is shifted towards smaller wavelengths featuring a zero phonon line located at $550 \mathrm{~nm}$. Its fluorescence lifetime of $\tau_{0}^{\mathrm{ST} 1} \approx$ $9.5 \mathrm{~ns}$ is shorter by about $3 \mathrm{~ns}$ [77]. The emission side band of the ST1 center at room temperature is rather broad and homogeneous, such that depletion at $775 \mathrm{~nm}$ is expected to be possible. Hence, the very same experimental apparatus can be utilized for super-resolving imaging of NV centers and ST1 centers.

At zero magnetic field, the ODMR spectra shows three resonances at $0.278 \mathrm{GHz}, 0.996 \mathrm{GHz}$ and $1.274 \mathrm{GHz}$ with a contrast of up to $45 \%$. Limited by the microwave amplifier bandwidth, the experimental system is not capable of efficiently performing coherent spin manipulation experiments on ST1 centers in the current configuration. However, by exchanging minor components of the driving field delivery system, the application of microwaves in the required frequency regime can be enabled for future applications. The observed ODMR spectrum is described by an electronic level structure featuring a singlet ground state and a metastable triplet state [77]. Singlet ground states prevents decoupling from the spin environment, which is advantageous in comparison to the NV center featuring a triplet ground state. No indication of a hyperfine coupling of the electron spin is observed in the ODMR spectrum, suggesting a nuclear-spin-free constituent. The absence of inherent close-by nuclear spins make the ST1 center a promising candidate for solely electron spin based QIP, as well as QS architectures. Possible candidates for the constituent include oxygen $\left(\mathrm{O}^{16}\right)$, magnesium $\left(\mathrm{Mg}^{24}\right)$ and calcium $\left(\mathrm{Ca}^{40}\right)$. 

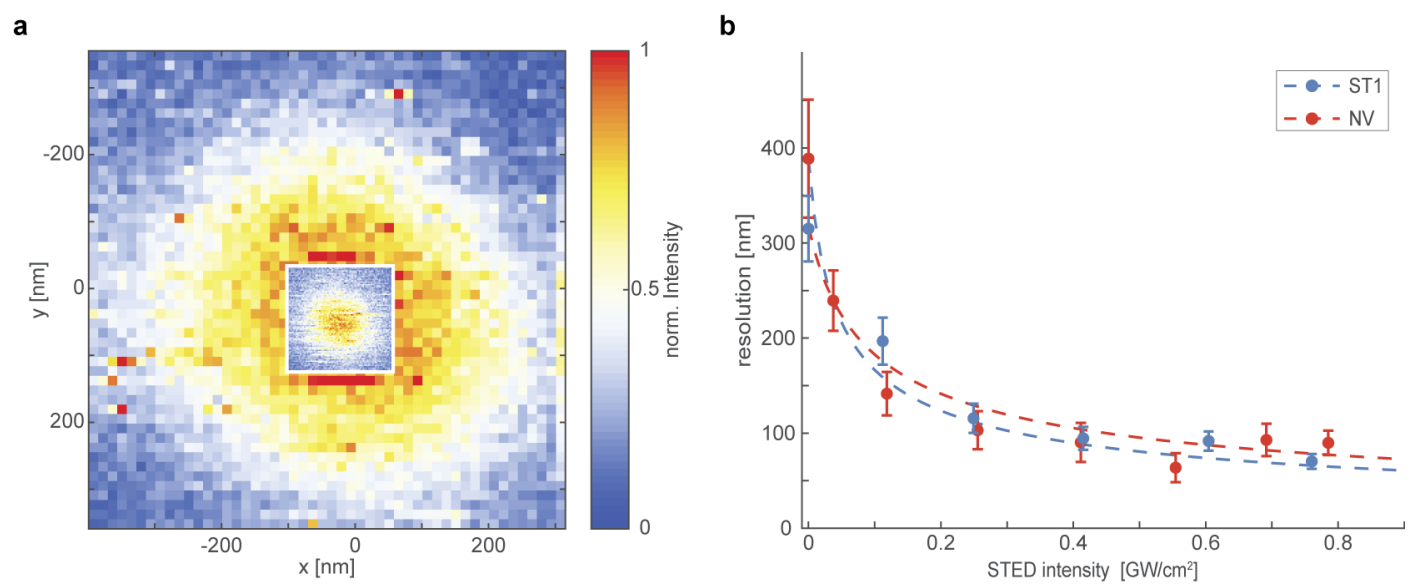

Figure 6.1.: STED imaging of a ST1 center. (a) Confocal and super-resolved (inset) image of a ST1 center in diamond. (b) Optical resolution in terms of the FWHM as a function of the applied STED intensity for ST1 (blue) and NV center (red), respectively. Colored dashed lines represent the fit of the super-resolution law.

\subsection{STED imaging}

Despite the slightly different optical properties, we utilize the experimental apparatus optimized for the imaging of NV centers (Ch. 5) for first proof-of-principle demonstration of super-resolution imaging on ST1 centers. The considered high pressure, high temperature (HPHT) diamond sample did not contain any fluorescent defects prior implantation. Negatively charged ions of energy $53 \mathrm{keV}$ and mass $27 \mathrm{u}$ (corresponding to $\mathrm{CN}^{-}$) were implanted under normal incident angle in the attempt to generate $\mathrm{NV}$ centers. After annealing at $800^{\circ} \mathrm{C}$ for $2 \mathrm{~h}$ in vacuum a random distribution of ST1 centers was observed. The sample was provided by our collaborators from the university of Leipzig.

In Fig. 6.1.a, the resolution enhancement of an image taken with $610 \mathrm{~mW}$ optical STED power (inset) compared to a confocal image of the same ST1 defect center is presented. Here, the resolution is limited by the damage threshold of the optical fibre delivering the STED beam. The obtained PSFs are fitted by a two-dimensional Gaussian of the form

$$
I(x, y)=I_{0} \exp \left[\left(x-\mu_{x}\right)^{2} /(2 \sigma)+\left(y-\mu_{y}\right)^{2} /(2 \sigma)^{2}\right]+O
$$

where $I_{0}$ is the amplitude, $O$ the background level, $\mu_{x}$ and $\mu_{y}$ the center position and $\sigma$ the standard deviation. The full-width at half-maximum (FWHM) of the intensity distribution is determined according to $\mathrm{FWHM}=2 \sqrt{2 \log 2} \cdot \sigma$. As a result, the confocal resolution $\mathrm{FWHM}_{\text {confocal }}=312 \pm$ $46 \mathrm{~nm}$ could be significantly improved by STED microscopy to $\mathrm{FWHM}_{\mathrm{STED}}=70 \pm 8 \mathrm{~nm}$. However, in order to push the resolution to its ultimate limits a depletion laser shifted towards the blue, where the depletion is expected to be more efficient, should be considered.

In Fig. 6.1.b the FWHM is plotted versus the STED intensity, which was varied between 0 and $0.76 \mathrm{GW} \mathrm{cm}^{-2}$ (blue). STED intensities are estimated by the spatio-temporal average of the applied optical STED power. Here, the intensity of the STED beam in the focal plain is approximated by [110]:

$$
I_{\mathrm{STED}}=\frac{P_{\mathrm{STED}}}{2.72 \cdot \pi \cdot \mathrm{FWHM}^{2} / 4}=\frac{I_{\mathrm{Gauss}}}{2.72} .
$$


Table 6.1.: Defect center properties. Experimentally obtained confocal $d_{\text {confocal }}$ and STED $d_{\text {STED }}$ resolution, STED beam saturation intensity $I_{\text {sat }}$ and STED beam cross section $\sigma_{\text {STED }}$ for NV and ST1 center. Additionally, the literature values of the fluorescence lifetime $\tau_{0}$ is given [30, 77].

\begin{tabular}{lcc} 
& $\mathrm{NV}$ & $\mathrm{ST} 1$ \\
\hline \hline$d_{\text {confocal }}[\mathrm{nm}]$ & $388.1 \pm 62.0$ & $317.4 \pm 34.4$ \\
$d_{\text {STED }}[\mathrm{nm}]$ & $78 \pm 11$ & $70 \pm 8$ \\
$I_{\text {sat }}\left[\mathrm{GW} / \mathrm{cm}^{2}\right]$ & $0.022 \pm 0.0089$ & $0.050 \pm 0.0235$ \\
$\sigma_{\text {STED }}\left[\mathrm{cm}^{2}\right]$ & $(8.51 \pm 3.4) \cdot 10^{-17}$ & $(3.62 \pm 1.7) \cdot 10^{-17}$ \\
$\tau_{0}[\mathrm{~ns}]$ & 12 & 9.5
\end{tabular}

The factor 2.72 is theoretically derived and scales the Gaussian FWHM to its doughnut shaped counterpart. Subsequently, the super-resolution law [89]

$$
d_{\mathrm{STED}}=d_{\text {confocal }} \frac{1}{\sqrt{1+\frac{I_{\mathrm{STED}}}{I_{\mathrm{sat}}}}},
$$

is fitted to the data (dashed blue line), extracting the saturation intensity.

\subsubsection{Comparison to the NV center}

The resolution scaling of the ST1 center is compared to the one of an NV center within a diamond sample featuring similar properties. Nitrogen ions $\left({ }^{15} N^{+}\right)$were implanted by a nanoimplanter $[111,73]$ with an energy of $5 \mathrm{keV}$ generating NV centers shallowly underneath the surface after subsequent annealing. Utilizing the same experimental apparatus, the scaling of the optical resolution as a function of the applied STED intensity is recorded. The data is evaluated as described above and presented in Fig. 6.1.b (red).

Based on the rate equation of a simplified two-level system a formula connecting the STED saturation intensity to the depletion cross section can be derived [112]:

$$
I_{\mathrm{sat}}=\frac{\sigma_{\mathrm{exc}}}{\sigma_{\mathrm{STED}}} \frac{h \nu_{\mathrm{STED}}}{h \nu_{\mathrm{exc}}} I_{\mathrm{exc}}+k_{f l} \frac{h \nu_{\mathrm{STED}}}{\sigma_{\mathrm{STED}}} .
$$

Both resolution scaling curves were measured with the same experimental apparatus such that the photon energies $h \nu_{\text {exc }}$ and $h \nu_{\text {STED }}$ are identical. The point-like nature of defects in diamond suggests similar cross sections for excitation $\sigma_{\text {exc }}$ and depletion $\sigma_{\mathrm{STED}}$ of both of the defect centers. The fluorescence rate scales with the fluorescence lifetime $k_{f l}=\frac{1}{\tau_{0}}$, which is known to be similar for ST1 center and NV center. Due to the similarity of the optical properties the resolution scaling with the applied STED intensity is expected to be in the same order of magnitude. This is in good agreement with the experimental observation. Table 6.1 summarizes the fit values and reconstructed depletion cross sections for ST1 and NV center.

\subsubsection{Summary}

In this chapter the super-resolution STED imaging of the ST1 center in diamond was demonstrated. Measurements were performed on an experimental apparatus optimized for the imaging of NV centers. The obtained resolution of $\sim 70 \mathrm{~nm}$ represents a fivefold improvement compared to confocal imaging mode and was limited by the available STED beam intensity. The scaling of the 
resolution with the STED intensity is comparable to the one of NV centers, suggesting that the use of optimized ST1 center diamond samples enables optical resolutions in the regime of a few nanometers [43].

Due to the technical challenges in the generation of dipole-dipole coupled NV center pairs it is advisable to search for additional defect centers in solids with similar physical properties but allowing easier implantation. Towards this end, the ST1 center proofs the availability of additional defect centers featuring ODMR, even though for the moment its constituent remains unidentified. 


\section{Fluorescent nuclear track detection}

Fluorescent nuclear track detectors (FNTDs) in combination with STED microscopy enable new insights in radiation therapy and the determination of physical properties. Here, the ability to determine the vacancy diffusion coefficient in diamond with surpassing precision is discussed based on the concept presented in [75] (Sec. 7.2). This property is of fundamental importance in the creation of optimized samples for quantum information processing (QIP) and quantum sensing (QS) applications. Additionally, the imaging of sub-cascade events is described (Sec. 7.2.1).

\subsection{Sample description}

The analysed sample was prepared by collaborators from the Tsukuba University, Japan, utilizing a HPHT diamond sample with an nitrogen abundance of $10 \mathrm{ppb}$. Irradiation with osmium (Os) ions was performed at an energy of $490 \mathrm{MeV}$ and a flux of $1 \times 10^{7} \mathrm{ions} / \mathrm{cm}^{2}$ under an incidence angle of $0^{\circ}$. Annealing was conducted at $1000^{\circ} \mathrm{C}$ for $2 \mathrm{~h}$ in vacuum. Imaging from the side perpendicular to the osmium ions direction of travel, enables scanning of the sample within a single focal depth. In that way, a varying imaging quality due to depth induced aberration can be avoided. In Fig. 7.1.a a typical confocal overview of an ion trace with an approximate length of $12 \mu \mathrm{m}$ is presented. Based on simulations performed with "Stopping and range of ions in matter" (SRIM [113]), the total number of vacancies within an average ion trace is estimated to be in the order of 35, 000 .

The two processes contributing to the loss of energy of the heavy ion are electronic and nuclear stopping. Electronic stopping predominantly occurs for high ion energies. Typically, the energy transferred to the electron is not sufficient to displace a carbon atom out of the diamond lattice. In contrast, for slow ions $(\sim 10 \mathrm{keV})$ the interaction with nuclei of the crystal lattice become dominant which displaces a carbon atom out of the lattice if the energy transfer is higher than the binding energy. Consequentially, close to the Bragg peak the generation of vacancy is most likely and highest vacancy densities are expected. Lower vacancy densities closer to the entrance point of the ions enables a mechanism to estimate the vacancy diffusion coefficient in diamond [75].

\subsection{Vacancy diffusion coefficient in diamond}

The diffusion coefficient of carbon and nitrogen within diamond have been subject of intense studies [114] and the obtained knowledge could be used to optimize the curing of the diamond lattice after damaging [115]. Even though the mobilization of vacancies (GR1 center) above $600{ }^{\circ} \mathrm{C}$ is observed [116], only recently the direct determination of the vacancy diffusion coefficient utilizing FNTD has been reported [75]. Here, a further development of this principle replacing the confocal by STED microscopy is discussed with respect to its capability to extract the diffusion coefficient with higher precision.

Close to the entrance point high energetic osmium ions displace carbon atoms from the crystal lattice with small but finite probability, thereby generating vacancies. Due to the conservation 

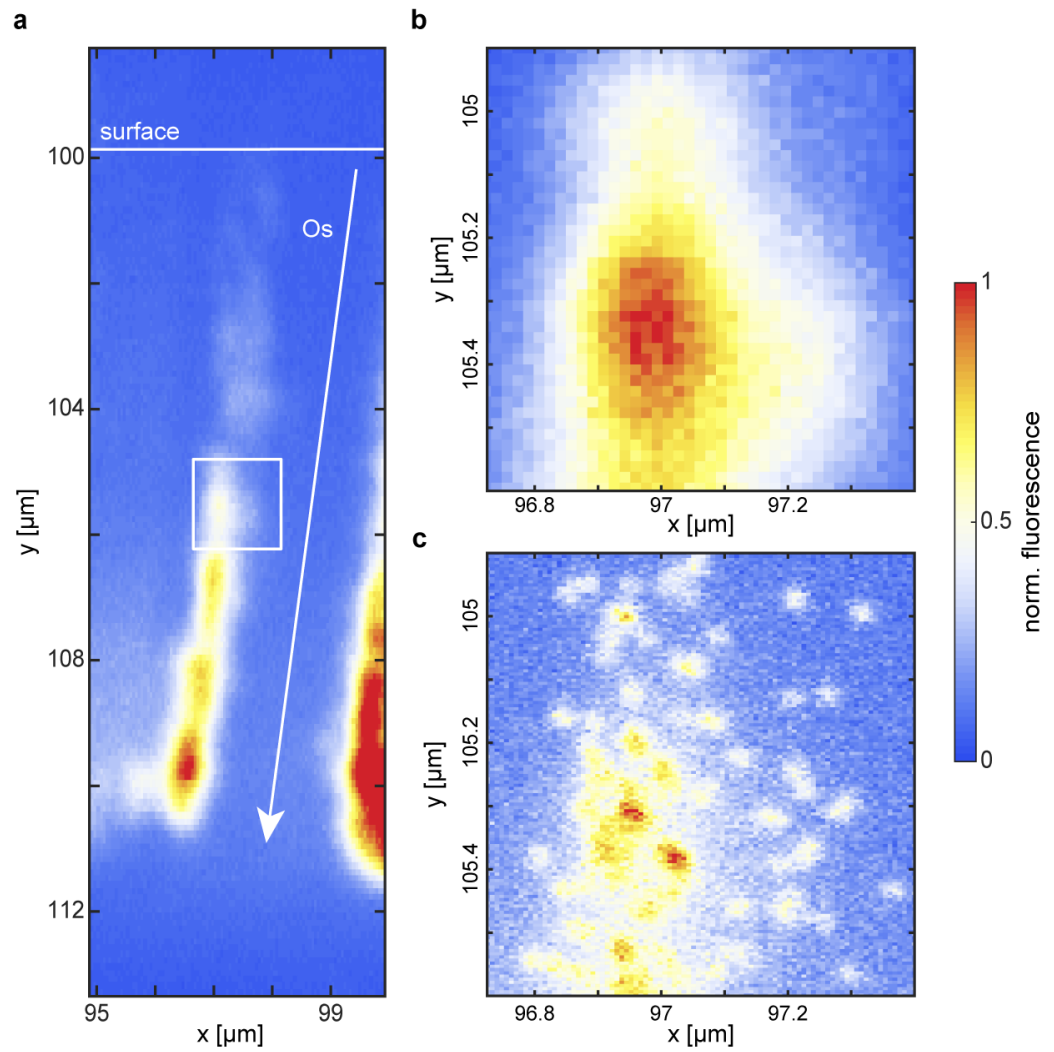

Figure 7.1.: High-resolution ion track imaging. (a) Confocal overview image of a complete fluorescent ion track. (b) Enlargement of the area within the white rectangular in (a). (c) STED imaging of the same region as in (b) reveals single defect centers.

of momentum the interaction with the carbon diamond lattice does not redirect the osmium ions and therefore, vacancies are created along a well-defined, narrow line. For this reason the presented analysis focuses on the region indicated by the white rectangular in Fig. 7.1.a. Annealing mobilizes the vacancies and generates NV centers, whenever a vacancy is caught by one of the (in first approximation) homogeneously distributed nitrogen atoms. The evolution of the NV center distribution as a function of annealing temperature and time contains information on the lateral vacancy diffusion coefficient in diamond [75]. Obviously, conventional microscopy techniques are incapable of resolving the NV center distribution within a diffraction limited spot. An approximation might be obtained by estimating the number of NV centers utilizing the integrated intensity and knowing the average distance of the nitrogen atoms within the diamond lattice. In contrast, super-resolution microscopy offers the potential to determine the vacancy diffusion coefficient via the exact number and positions of the NV centers.

In parts (b) and (c) of Fig. 7.1 the comparison between a standard confocal image and the superresolved STED image within the region of interest is shown. At an optical stimulated emission depletion beam power of $650 \mathrm{~mW}\left(I_{\mathrm{STED}} \sim 0.81 \mathrm{GW} \mathrm{cm}^{-2}\right)$ single nitrogen vacancy centers are observed. Currently, the optical resolution of roughly $50 \mathrm{~nm}$ is limited by the available STED beam power.

\subsubsection{Resolution}

Figure 7.2 presents the obtained resolution (FWHM) as a function of the optical STED intensity for a shallow ion trace (approx. $1 \mu \mathrm{m}$ underneath the surface). A fit of the expected scaling behaviour 


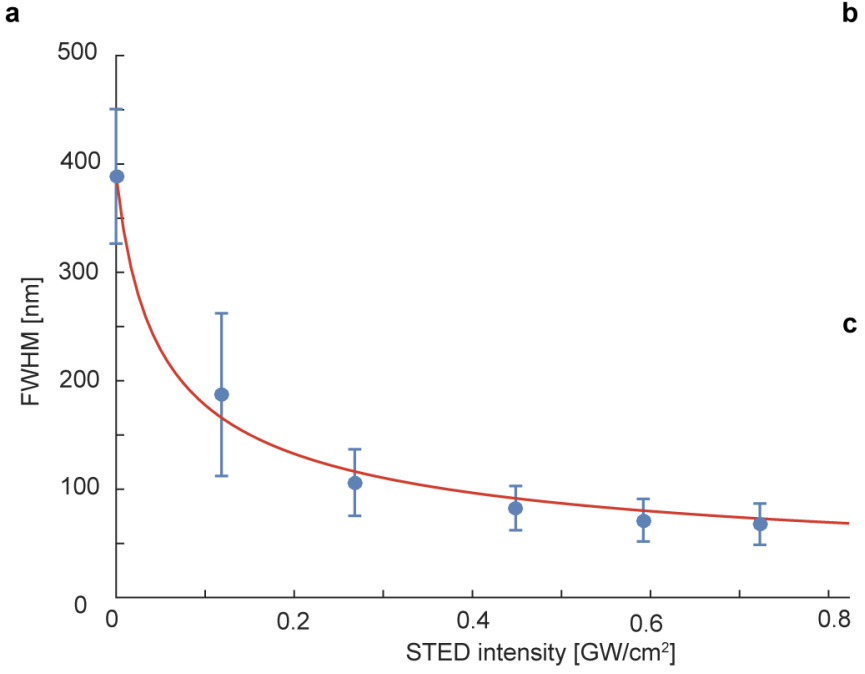

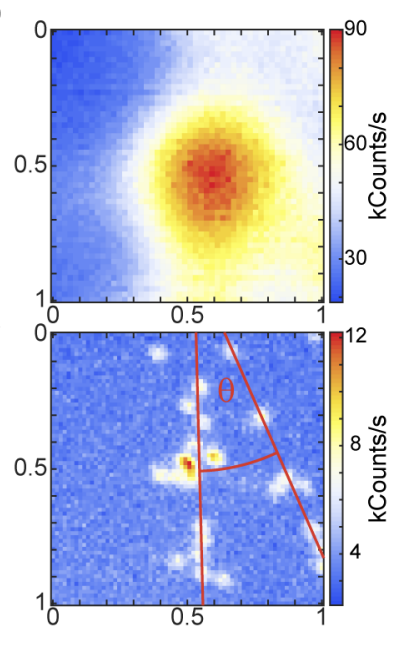

Figure 7.2.: Ion track resolution. (a) Optical resolution as a function of the applied STED intensity. The red line represents a fit of the super-resolution law. (b-c) Confocal and STED image of the same cascade event, respectively. The high-resolution image enables the extraction of the divergence angle $\theta \approx 22^{\circ}$ of a cascade event.

(Eq. 4.2) is shown as a red solid line. The confocal resolution of FWHM $=388.6 \pm 62.0 \mathrm{~nm}$ was improved to FWHM $=67.7 \pm 19.1 \mathrm{~nm}$ at a STED intensity of $0.722 \mathrm{GW} \mathrm{cm}^{-2}$. Since primarily single NV centers are observed, the resolution is sufficient for the anticipated application. Additionally, the observation of cascade events is enabled (Fig. 7.2.b-c). While the confocal image displays a single bright spot, the STED image shows two distinct lines diverging under an angle of $\theta \approx 22^{\circ}$. Such events are predicted by SRIM-simulations [75] and contain information on the absorption of particles of high-energy in matter.

\subsubsection{Localization}

In order to localize emitters in Fig. 7.1.c in an automatized fashion the evaluation routine presented in [117] was adopted. First, the resolution is estimated by fitting a two-dimensional Gaussian function $G(x, y)$ to a well isolated NV center. Fit values are assumed to represent the idealized PSF and contain information on the obtained resolution and integrated intensity of a single emitter. Subsequently, a threshold analysis performed on the cross correlation of the idealized PSF with the raw image, pre-locates the emitters. In a small area around each localization, a two-dimensional Gaussian fit to the original STED image is performed in order to increase the localization precision. The integrated intensity in 16 out of the 90 localization areas indicates the presence of two or more NV centers and is utilized as an estimate for the exact number of emitters [117]. The obtained resolution is not sufficient to resolve all NV centers individually. For simplicity it is assumed that all contributing emitters within a localization region are located in the center of the Gaussian fit. In this way, introduced spatial uncertainties are less than or equal to the optical resolution $(\sim 50 \mathrm{~nm})$ and should not influence the overall emitter distribution significantly.

\subsubsection{Binning}

Based on the localizations the Euclidean distance of each NV center to the ion track is determined. The most probable ion trajectory is estimated by determining the center of mass $C_{x}(y)$ along the $\mathrm{x}$-direction for each line $\mathrm{y}$ in the confocal image. Subsequently, a straight line is fitted to 


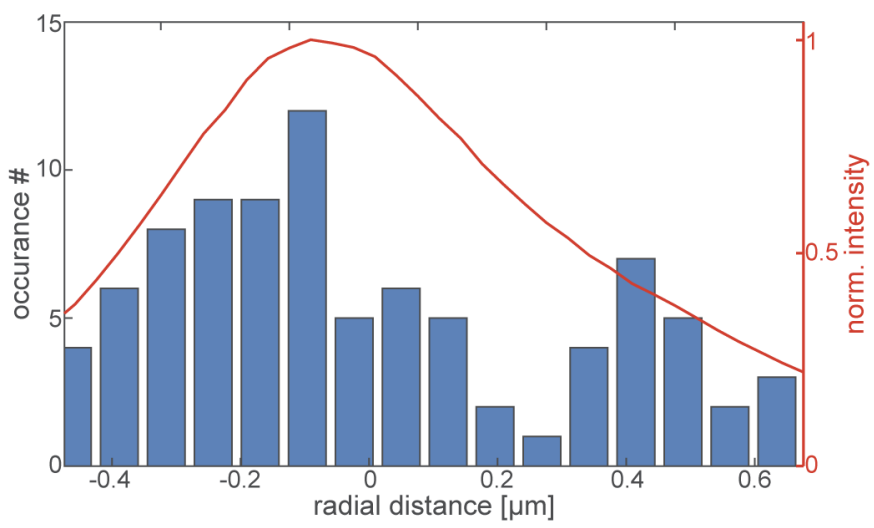

Figure 7.3.: NV center distribution within an ion trace. Histogram of the NV center distribution determined from super-resolution imaging (blue) with respect to the center of mass of the ion track. The histogram of the NV center position extracted from the STED image reveals the substructure hidden in the along the ion track integrated confocal intensity distribution (red line).

$C_{x}(y)$. The histogram shown in Fig. 7.3 presents the number of localizations as a function of the distance to the ions trajectory. Additionally, the integrated fluorescence along the ion trace of the confocal image is presented (red line). While the confocal intensity distribution shows a single asymmetric peak, the NV center distribution obtained by STED microscopy reveals the substructure consisting of two distinct peaks. The increased localization precision will enable the determination of the vacancy diffusion coefficient with high precision in the future.

\subsection{Summary}

In this chapter, the indirect STED imaging of the trajectory of osmium ions of high-energy within diamond was presented An optical resolution of $50 \mathrm{~nm}$ could be obtained, which is remarkable considering that the ion track is located more than $1 \mu \mathrm{m}$ underneath the diamond surface. The obtained resolution was limited on the one hand by the accessible optical STED power on the other by depth induced aberrations. However, imaging single NV centers within FNTDs with high statistics will in future enable the precise determination of the vacancy diffusion coefficient in diamond. To accomplish this, the measurements need to be repeated for samples with varying annealing parameter (temperature and time). Limited by the availability of suitable specimen we were restricted to the presentation of proof-of-principle measurements. The determination of the vacancy diffusion coefficient represents a crucial step for improving the conversion yield of implanted nitrogen ions into NV centers. An increased conversion yield represents an important step towards scalable deterministic creation of solid-state spin based quantum registers. Additionally, the possibility to resolve cascade events might provide a means for new insights in radiation therapy. 
Quantum information processing 



\section{Geometric phase based quantum gates}

Soon after their discovery geometric phases were counted as a potentially powerful tool for robust quantum computation. First an intuitive classical example is given, highlighting some of the features of geometric phases. Afterwards, a closer look on the mathematical representation of the Berry phase and its generalizations to Aharonov-Anandan and holonomic geometric phases is taken. Additionally, the origin of the intrinsic robustness is discussed.

\subsection{Intuitive example}

In general, geometric phases arise when parallel transporting (meaning without any local rotation) a vector on a surface with non-trivial geometry. An intuitive classical illustration is shown in Fig. 8.1.a. A vector (blue) located at the pole of a 2-sphere (I) (in this example this is not the Bloch-sphere) is parallel transported to the equatorial plain $(I I)$ and subsequently along the equatorial line $(I I I)$. Finally, the trajectory is closed by transporting the vector back to the pole $(I V)$. Even though no local rotation has been performed, the vectors at position $(I)$ and $(I V)$ differ by an angle $\gamma$. This angle $\gamma$ is a consequence of the non-trivial geometry of the sphere surface and is solely depending on the solid angle $\Omega$ (red shaded area) enclosed by the circuit $C$ (red solid line). Hence, sometimes geometric phases are referred to as a global change without a local change. You can easily convince yourself that the cyclic parallel transport of a vector on a surface of trivial (flat) geometry will not generate such an additional phase factor (Fig. 8.1.c).

Similarly the origin of the robustness property can be illustrated (Fig. 8.1.b). Imagine, due to noise or experimental control parameter imperfections, the vector is not following the anticipated trajectory (red line) exactly but is jittering around it (blue line). As mentioned above, the final value of $\gamma$ is only depending on the enclosed solid angle $\Omega$ such that the jitter is averaging out as long as the noise amplitude is small and its frequency high with respect to the inverse gate duration $1 / t_{\text {Gate. }}$. Hence, geometric phases feature an intrinsic tolerance against noise.

\subsection{Berry phase}

In 1984, M.V. Berry discovered that an additional phase factor is associated with a cyclic adiabatic system evolution in parameter space, which only depends on the geometry of the Hilbert space [78]. Let $H$ be a Hamiltonian depending on the parameter set $\mathbf{R}$ and let $\mathbf{R}$ perform an adiabatic cycle such that $\mathbf{R}(T)=\mathbf{R}(0)$. The instantaneous eigenstates $|n(R(t))\rangle$ depend on the chosen path in parameter space. For a system initialized in one of the eigenstates $|n(R(0))\rangle$ under the adiabatic condition the final state $|\Psi(t)\rangle$ at time $t$ follows as:

$$
|\Psi(t)\rangle=\exp \left(-\frac{i}{\hbar} \int_{0}^{t} E_{n}\left(R\left(t^{\prime}\right)\right) \mathrm{d} t\right) \exp \left(i \gamma_{n}(t)\right)|n(R(t))\rangle .
$$


a

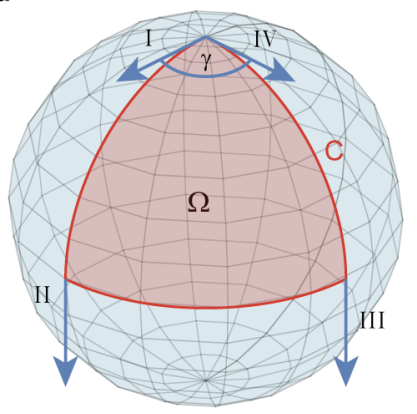

b

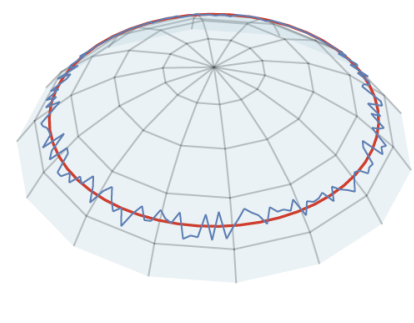

c

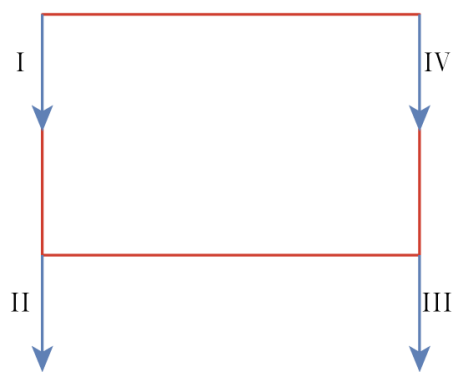

Figure 8.1.: Classical example of a geometric phase. (a) Intuitive explanation of the origin of geometric phases. A vector (blue arrow) is transported along a closed loop trajectory (red line) without any local rotation (parallel transport condition). The non-trivial geometry of the 2-sphere surface results in a global change $\gamma$ of the vector without a local change. (b) Fast noise of small amplitude does not alter the geometric phase $\gamma$ significantly since $\gamma$ only depends on the enclosed solid angle. (c) In contrast, when parallel transporting a vector in a closed loop on a trivial (flat) surface the local and global properties of the vector remain unchanged.

Next to the dynamic phase (first exponential) a second phase factor $\gamma_{n}(t)$ arises. Under the cyclic condition the geometric phase follows as a circuit integral

$$
\gamma_{n}(C)=-i \oint_{c}\left\langle n(R) \mid \nabla_{R} n(R)\right\rangle \mathrm{d} R
$$

where $C$ is the closed path in parameter space $\mathbf{R}$. It follows that $\gamma_{n}(C)$ only depends on the path of $\mathbf{R}$ as long as the evolution is performed slow enough. Since $\left\langle n(R) \mid \nabla_{R} n(R)\right\rangle$ is imaginary the geometric phase factor $\gamma_{n}(C)$ is real valued. Utilizing Stokes theorem converts the circuit integral into a surface integral

$$
\gamma_{n}(C)=-\iint \mathrm{d} S \cdot V_{n}(R)
$$

where $\mathrm{d} S$ is the surface area element in parameter space $R$ and $V_{n}(R)$ is referred to as vector potential since it originates from the curl of a vector. As the intuitive example in Sec. 8.1 the geometric phase only depends on a surface integral.

\subsection{Aharonov Anandan phase}

It were Y. Aharonov and J. Anandan who realized that the occurrence of geometric phases is not restricted to adiabatic system evolutions [118]. In fact, every closed curve in the projective Hilbert space gives rise to a geometric contribution to the total phase $\phi$, which is derived to be:

$$
\phi=\underbrace{-\int_{0}^{T}\langle\psi(t)|H(t)| \psi(t)\rangle \mathrm{d} t}_{\text {Dynamic }}+\underbrace{\int_{0}^{T}\left\langle\widetilde{\psi}(t)\left|i\left(\partial / \partial_{t}\right)\right| \widetilde{\psi}(t)\right\rangle \mathrm{d} t}_{\text {Geometric }} .
$$

Here, the first term is again the well known dynamic phase equivalent to the one in Eq. 8.1 and the second is the geometric contribution referred to as Aharonov-Anandan (AA) phase. Note, $|\widetilde{\psi}(t)\rangle$ is the periodic state vector representation of the cyclic state sometimes referred to as reference section state [119]. However, in general the AA phase is restricted to non-commuting (Abelian) 
$U(1)$ transformations. Obviously, in the adiabatic limit the AA phase corresponds to the Berry phase.

\subsection{Abelian non-adiabatic geometric quantum gates}

The Aharanov-Anandan phase is only a promising tool for geometric phase based quantum information applications if the dynamic phase contribution can be suppressed efficiently. Typically, the so called two-loop method, where the dynamic phase acquired in the first half of the sequence cancels with the one acquired during the second (used, e.g., in [120, 121, 122]), is utilized. Hence, the dynamic contributions are self-compensating and pure geometric evolution is achieved. Note, even though the value of the dynamic phase at the end of the sequence is zero, it is not during the gate operation itself.

\subsection{Non-Abelian holonomies}

A conceptually different approach to geometric phase based quantum computation was suggested by P. Zanardi and M. Rasetti [123]. Its main advantage is the possibility to generate $S U(2)$ (matrix valued) transformations resulting in the realization of non-commuting (non-Abelian) quantum gates by means of holonomies [124]. Essentially, a computational subspace is adiabatically transported on a closed loop in a higher dimensional Hilbert space.

However, this approach requires at least a four level system $n \geq 4$ with at least three driving fields [123]. The high number of experimental control parameter in combination with its adiabatic nature hindered the experimental demonstration of this quantum gate concept for more than a decade. Only recently, adiabatic single-qubit holonomic quantum gates have been demonstrated in a trapped ion four-level tripod configuration [125].

It is noteworthy, that holonomy based quantum gates allow to acquire the geometric phase by the evolution of a computational subspace with energy eigenvalue zero [126]. Hence, the acquired dynamic phase is equal to zero at any point during the sequence and pure geometric evolution is obtained by default. However, strict timing conditions need to be fulfilled in order to avoid loses into the ancillary levels.

\subsection{Non-adiabatic non-Abelian holonomies}

In 2012, E. Sjöqvist et al. proposed a scheme for non-adiabatic non-Abelian geometric quantum computation overcoming the need for long gate durations [127]. The availability of a set of purely geometric, universal, non-adiabatic quantum gates drew significant attention and was experimentally realized utilizing microwave [61, 60] and optical transitions [128, 129] on nitrogen-vacancy center in diamond, as well as in liquid NMR [79], super-conducting qubits [80].

Consider the $\mathrm{V}$-shaped level scheme featuring the ground state $|0\rangle$ and the two excited states $| \pm\rangle$. The computational basis spanned by $|+\rangle$ and $|-\rangle$, while $|0\rangle$ acts as in ancilla. The resonantly driven interaction Hamiltonian in the rotating is defined by:

$$
H(t)=\Omega(t) / 2\left(\gamma_{-}|0\rangle\left\langle-\left|+\gamma_{+}\right| 0\right\rangle\langle+|+\text { H.c. }\right)
$$

Resulting in a Rabi oscillation between the ground $|0\rangle$ and bright state $|b\rangle=-\gamma_{+}^{*}|+\rangle-\gamma_{-}^{*}$, while the dark state $|d\rangle=-\gamma_{-}|+\rangle+\gamma_{+}|-\rangle$decouples from the gate operation [130]. Since $\Omega(t)$ is 
the Rabi frequency the systems performs a cyclic evolution if $\int_{0}^{t^{\prime}} \Omega\left(t^{\prime}\right) \mathrm{d} t^{\prime}=2 \pi$, in case the Rabi weights are well normalized $\left|\gamma_{-}\right|^{2}+\left|\gamma_{-}\right|^{2}=1$. The final evolution operator only depends on the chosen path $C_{n}$

$$
U^{(1)}\left(C_{n}\right)=\mathbf{n} \cdot \vec{\sigma}
$$

where $\vec{\sigma}$ is the vector of the standard Pauli matrices $\vec{\sigma}=\left(\sigma_{x}, \sigma_{y}, \sigma_{z}\right)$. For $\gamma_{-}=\sin (\theta / 2) e^{i \phi}$ and $\gamma_{+}=-\cos (\theta / 2)$ the unit vector $\mathbf{n}$ follows as:

$$
\mathbf{n}=(\cos (\theta) \sin (\phi), \sin (\theta) \sin (\phi), \cos (\theta))
$$

By choosing the components $\theta$ and $\phi$ appropriately (which defines the Rabi frequency ratio $\gamma_{+} / \gamma_{-}$) the rotation axis of the operation $U^{1}\left(C_{n}\right)$ is defined. Rotations by arbitrary angles can be obtained by subsequent application of $\pi$-pulses around the unit vectors $\mathbf{n}$ and $\mathbf{m}$, such that:

$$
U^{(1)}(C)=U^{(1)}\left(C_{m}\right) U^{(1)}\left(C_{n}\right)=\mathbf{n} \cdot \mathbf{m}-i \sigma(\mathbf{n} \times \mathbf{m}) .
$$

$U^{(1)}(C)$ is an $S U(2)$ transformation, equivalent to a rotation around the normal of the plain spanned by $\mathbf{n}$ and $\mathbf{m}$ by an angle of $2 \arccos (\mathbf{m} \cdot \mathbf{n})$. Consequentially, $U^{(1)}(C)$ is a universal single qubit quantum gate. A similarly derived two-qubit (conditional phase shift) quantum gate completes the universal non-adiabatic holonomic quantum gate set [127].

\subsection{Intrinsic robustness}

On the one hand, geometric phases provided understanding of fundamental physics phenomena like the Aharonov-Bohm effect [131] and are in itself a fascinating field of research. On the other, they are a promising tool to realize robust high fidelity quantum gates as building blocks for NISQ. The reason for the conjecture that geometric phase based quantum gates are resilient against noise is that they rely on global features while decoherence and parameter noise are of local nature.

The influence of noise on geometric phase based quantum gates has been investigated theoretically and experimentally in certain scenarios. E.g., S. Filipp et al. demonstrated the resilience against artificial noise for adiabatic geometric phase shift gates with increasing gate duration [132, 133]. Naively, on might expect the longer exposure to the noise source reduces the gate fidelity. However, by prolonging the gate duration, the noise frequency in comparison to the inverse gate duration is increased and the effect of the noise is more likely to average out [134, 135] (compare Sec. 8.1). Consequentially, quasi static (DC) noise is distorting the path and results in dephasing, which however appears to be less pronounced than for the dynamic counterpart [136].

Numeric investigations by M. Johansson et al. suggest superior robustness against decay, dephasing and constant control parameter imperfections of non-adiabatic holonomic quantum gates with respect to their adiabatic counterparts [137]. Similarly, Liang et al. proposed supremacy of the superadiabatic geometric quantum gate (realized in Ch. 11) over non-adiabatic holonomic and adiabatic geometric quantum gates with respect to constant driving field miss tuning [81].

In conclusion, there are strong indications that geometric phase based quantum gates are robust with respect to local noise and constant driving field parameter imperfections. However, a detailed comparative study on the performance of different quantum gate modalities based on dynamic and geometric phases has not yet been performed. 


\section{Performance evaluation}

Commonly, two different approaches for the quantification of the performance of quantum gates are followed. Standard quantum process tomography (QPT) reconstructs the quantum process matrix of single gate operations. Hence, it enables the identification and optimization of errorprone gates. In contrast, randomized benchmarking $(\mathrm{RB})$ reveals the error scaling of Clifford gate based computational sequences of different length. While it extracts the computational relevant error, it does not contain information on the individual gate performance. In the scope of this thesis QPT and RB are implemented to reveal the complete performance information.

\subsection{Standard quantum process tomography}

Standard QPT is a commonly used tool (e.g., [138, 139, 140, 61]) in order to reconstruct the quantum process matrix $\chi_{\exp }$ of an unknown quantum operation. $\chi_{\exp }$ contains the full information on the process. The fidelity $F$, defined as the overlap of the unknown, experimentally measured and the ideal quantum process matrix $F=\operatorname{Tr}\left(\chi_{\exp } \chi_{\text {theo }}\right)$, quantifies the gate performance with $F=1$ for an ideal gate realization. QPT makes it possible to determine the quantum gate fidelity for different gates (e.g., Pauli-X, Pauli-Z) separately, revealing full performance information about the considered set of gates.

\subsubsection{Quantum process matrix}

The quantum process matrix $\chi$ describes how an arbitrary input state $|\psi\rangle$ transforms under an unknown quantum operation $\mathcal{E}$. An input state can be described by the density matrix according to

$$
\rho^{i n}=\sum_{k} p_{k}|k\rangle\langle k|
$$

with $0 \leq p_{k} \leq 1, \sum p_{k}=1$ and $|k\rangle$ are elements of the $d$-dimensional Hilbert space. The operation $\mathcal{E}$ acting on $\rho^{\text {in }}$ generates the output state $\rho^{\text {out }}=\mathcal{E}\left(\rho^{i n}\right)$. By switching to the operator-sum representation the output state can be described by the quantum process matrix $\chi_{n m}$ according to $[16]$

$$
\rho^{o u t}=\mathcal{E}\left(\rho^{i n}\right)=\sum_{m n} \chi_{m n} E_{m} \rho^{i n} E_{n}^{\dagger}
$$

where $E_{m} \rho^{i n} E_{n}^{\dagger}$ is a basis transformation into $\left\{E_{m}\right\} \in \mathcal{S U}(d)$, representing a full set of orthogonal basis operator.

\subsubsection{Evaluation routine}

The utilized quantum process tomography evaluation routine is based on the work presented in [141]. For completeness, a short summary of the general concept is given (for details see [142]). 
Measurements are performed on $d^{2}$ states $\Psi_{j}$ forming a orthogonal basis:

$$
\rho=\sum_{j} q_{j} \rho_{j}=\sum_{j} q_{j}\left|\Psi_{j}\right\rangle\left\langle\Psi_{j}\right|
$$

Both $\mathcal{E}\left(\rho_{j}^{i n}\right)$ and $E_{m} \rho^{i n} E_{n}^{\dagger}$ can be decomposed into the basis spanned by $\rho_{k}$ :

$$
\begin{aligned}
\mathcal{E}\left(\rho_{j}^{i n}\right) & =\sum_{k} \lambda_{j k} \rho_{k}, \\
E_{m} \rho^{i n} E_{n}^{\dagger} & =\sum_{k} \beta_{j k}^{m n} \rho_{k} .
\end{aligned}
$$

The result of $\mathcal{E}\left(\rho_{j}^{i n}\right)$ is evaluated by quantum state tomography measuring its components in the basis of $\rho_{k}$. This requires $d^{2}$ projective measurements per input state $\rho_{j}^{i n}$ resulting in a total of $d^{4}$ measurement. From the measurement outcomes $\mathcal{O}$ the coefficients $\lambda_{j k}$ are calculated by solving the linear system of equation:

$$
\mathcal{O}_{p j}=\left\langle\Psi_{p}\left|\mathcal{E}\left(\rho_{j}^{i n}\right)\right| \Psi_{p}\right\rangle=\sum_{k} \lambda_{j k}\left\langle\Psi_{p}\left|\rho_{k}\right| \Psi_{p}\right\rangle=\sum_{k} \lambda_{j k}\left|\left\langle\Psi_{p} \mid \Psi_{k}\right\rangle\right|^{2} .
$$

Similarly, the coefficients $\beta_{j k}^{n m}$ follow by solving the system of linear equations which is purely based on theoretical values:

$$
\left\langle\Psi_{p}\left|E_{m} \rho_{j}^{i n} E_{n}^{\dagger}\right| \Psi_{p}\right\rangle=\sum_{k} \beta_{j k}^{m n}\left\langle\Psi_{p}\left|\rho_{k}\right| \Psi_{p}\right\rangle=\sum_{k} \beta_{j k}^{m n}\left|\left\langle\Psi_{p} \mid \Psi_{k}\right\rangle\right|^{2} .
$$

After calculating the generalized inverse $\kappa_{j k}^{m n}$ of $\beta_{j k}^{m n}$ the experimental quantum process matrix follows from Eq. 9.2 immediately:

$$
\chi_{m n}=\sum_{j k} \kappa_{j k}^{m n} \lambda_{j k}
$$

However, due the imperfect measurement or dissipative processes and decoherence the calculated quantum process matrix is in general not physical, i.e., $\chi$ can be non-Hermitian, traceless or not completely positive. To this end, a maximum-likelihood-estimation is performed resulting in the most probable physical $\chi$-matrix [61]. The error-estimation is based on a Monte-Carlo simulation utilizing the mean and standard deviation of the observable $\mathcal{O}$, resulting in a distribution of MonteCarlo sampled, physical process matrix $\chi_{M C}$. The quantum gate fidelity and its error are calculated from the distribution of $\chi_{M C}$ matrices.

\subsubsection{Choice of basis operators}

In the scope of this thesis quantum gates defined on the two and three-dimensional Hilbert space $(d=\{2,3\})$ are demonstrated. Two sets of orthogonal basis operators $E_{m}$ need to be chosen to reconstruct the full quantum process matrices $\chi_{m m}$ in the respective Hilbert space. The chosen set of $E_{m}$ in two-dimensional Hilbert space spanned by the computational space $\{0,-\}$ are the well-known Pauli matrices completed by the identity matrix, the generators of the special unitary group $\mathcal{S U}(2)$. The most compact representation of generators $E_{m}$ spanning $\mathcal{S U}(3)$ are defined by the eight Gell-Mann matrices, which are Hermitian, traceless and fulfil $\operatorname{tr}\left(\lambda_{i} \lambda_{j}\right)=2 \delta_{i j}$. However, the Gell-Mann matrices do not allow to immediately extract the reduced $4 \times 4$ quantum process matrix of the computational subspace spanned by $\{+,-\}$. Here, we utilize the set of nine generators 
Table 9.1.: Definition of the QPT basis. Convention of the basis operators $E_{m}$ for quantum process tomography measurements in Pauli (P.), explicit state (exp.) and matrix (mat.) representation on the two and three-dimensional Hilbert space.

\begin{tabular}{|c|c|c|c|c|c|c|}
\hline$E_{m}$ & P. $\mathcal{H}^{2}$ & P. $\mathcal{H}^{3}$ & exp. $\mathcal{H}^{2}$ & exp. $\mathcal{H}^{3}$ & mat. $\mathcal{H}^{2}$ & mat. $\mathcal{H}^{3}$ \\
\hline$E_{1}$ & $\sigma^{0}$ & $\sigma_{+-}^{0}$ & $|0\rangle\langle 0|+|-\rangle\langle-|$ & $|+\rangle\langle+|+|-\rangle\langle-|$ & $\left(\begin{array}{ll}1 & 0 \\
0 & 1\end{array}\right)$ & $\left(\begin{array}{lll}1 & 0 & 0 \\
0 & 1 & 0 \\
0 & 0 & 0\end{array}\right)$ \\
\hline$E_{2}$ & $\sigma^{x}$ & $\sigma_{+-}^{x}$ & $|0\rangle\langle-|+|-\rangle\langle 0|$ & $|+\rangle\langle-|+|+\rangle\langle 0|$ & $\left(\begin{array}{ll}0 & 1 \\
1 & 0\end{array}\right)$ & $\left(\begin{array}{lll}0 & 1 & 0 \\
1 & 0 & 0 \\
0 & 0 & 0\end{array}\right)$ \\
\hline$E_{3}$ & $\sigma^{y}$ & $\sigma_{+-}^{y}$ & $i|0\rangle\langle-|-i|-\rangle\langle 0|$ & $i|+\rangle\langle-|-i|-\rangle\langle+|$ & $\left(\begin{array}{cc}0 & -i \\
i & 0\end{array}\right)$ & $\left(\begin{array}{ccc}0 & -i & 0 \\
i & 0 & 0 \\
0 & 0 & 0\end{array}\right)$ \\
\hline$E_{4}$ & $\sigma^{z}$ & $\sigma_{+-}^{z}$ & $|0\rangle\langle 0|-|-\rangle\langle-|$ & $|+\rangle\langle+|-|-\rangle\langle-|$ & $\left(\begin{array}{cc}1 & 0 \\
0 & -1\end{array}\right)$ & $\left(\begin{array}{ccc}1 & 0 & 0 \\
0 & -1 & 0 \\
0 & 0 & 0\end{array}\right)$ \\
\hline$E_{5}$ & - & $\sigma_{+0}^{x}$ & - & $|+\rangle\langle 0|+| 0\rangle\langle+|$ & - & 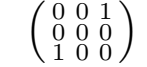 \\
\hline$E_{6}$ & - & $\sigma_{+0}^{y}$ & - & $-i|+\rangle\langle 0|-i| 0\rangle\langle+|$ & - & $\left(\begin{array}{ccc}0 & 0 & -i \\
0 & 0 & 0 \\
i & 0 & 0\end{array}\right)$ \\
\hline$E_{7}$ & - & $\sigma_{-0}^{x}$ & - & $|-\rangle\langle 0|+| 0\rangle\langle-|$ & - & $\left(\begin{array}{lll}0 & 0 & 0 \\
0 & 0 & 1 \\
0 & 1 & 0\end{array}\right)$ \\
\hline$E_{8}$ & - & $\sigma_{-0}^{y}$ & - & $-i|-\rangle\langle 0|-i| 0\rangle\langle-|$ & - & $\left(\begin{array}{ccc}0 & 0 & 0 \\
0 & 0 & -i \\
0 & i & 0\end{array}\right)$ \\
\hline$E_{9}$ & - & $I_{0}$ & - & $|0\rangle\langle 0|$ & - & $\left(\begin{array}{lll}0 & 0 & 0 \\
0 & 0 & 0 \\
0 & 0 & 1\end{array}\right)$ \\
\hline
\end{tabular}

$E_{m}$ suggested in [141]. Table 9.1 summarizes the choice of basis states $E_{m}$ in the Pauli matrix, explicit state and matrix representation for $d=2,3$, respectively. $E_{1}$ to $E_{4}$ correspond to the generators of the (reduced) computational space.

\subsubsection{Experimental sequence}

The QPT measurement is performed in $d^{4}$ experimental runs. Each run is initialized by optically pumping the NV center electron spin into the $|0\rangle$ state (Fig. 9.1). The application of $\pi / 2$ and $\pi$-pulses prepares the spin in one of the $d^{2}$ input (quasi-)pure states $\rho_{j}^{i n}=\left|\psi_{j}\right\rangle\left\langle\psi_{j}\right|$ (Tab. 9.1). Subsequently, the gate operation is performed resulting in the final state $\rho_{j}^{\text {out }}=\mathcal{E}\left(\rho_{j}^{\text {in }}\right)$. Projective readout pulses perform the back-projection of $\rho_{j}^{\text {out }}$ onto $E_{m}$ resulting in the observable $\mathcal{O}_{p j}$ required for the evaluation presented in Sec. 9.1.2. Finally, the population is optically readout by illumination with green light and simultaneous fluorescence detection.

With increasing dimensionality $d$ of the Hilbert space the QPT measurement becomes experimentally elaborate and its evaluation computationally intense. Practically, the execution of QPT becomes infeasible for large $d$. However, universal quantum computation is obtained for a set of two non-commuting single qubit and one non-trivial two-qubit gate. Hence, as long as the computational qubit is encoded in a low dimensional Hilbert space standard quantum process tomography represents a powerful tool for the quantification of the quantum gate performance.

\subsubsection{Rotating frame considerations}

In Sec. 12.4 the quantum gate fidelity of a gate operation suffering from the intentional detuning $\eta$ of the driving field frequency is investigated. In general, subsequent gate operations suffering the same constant detuning $\eta$ are performed in the identical rotating frame of reference. Consequentially, the on-resonant QPT preparation and projection pulses need to be applied in the reference frame of the imperfect gate operation. Experimentally this situation is achieved by properly switching between the involved rotating driving field frames (compare Sec. 10.1.1) as described in the following. 


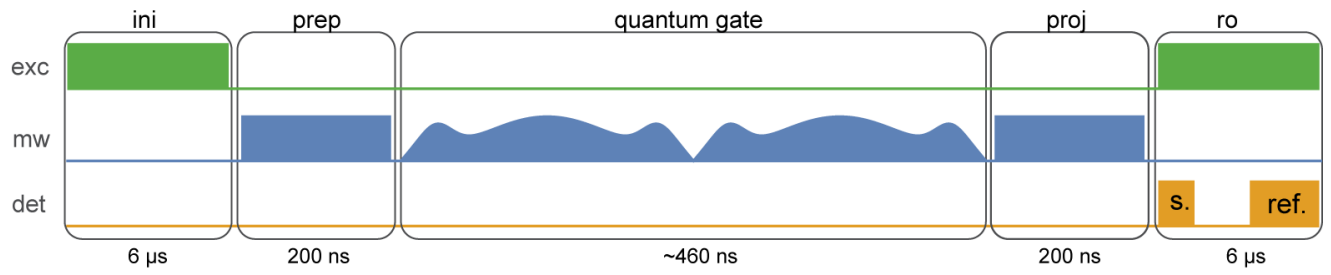

Figure 9.1.: Quantum process tomography measurement scheme. After initialization (ini) a preparation pulse (prep) is applied. Subsequently, the gate operation is performed. A projection pulse (proj) and finally the spin state is readout (ro). Each of the preparation pulses is combined with each of the projection pulses resulting in $d^{4}$ combinations, where $d$ is the dimension of the investigated Hilbert space.

By defining the time point $t=0$ between preparation and the gate operation a smooth transition (no phase jump) between the on-resonant (negative times) and the detuned (positive times) reference frame is ensured. However, at the end of the gate operation $t=t_{\text {Gate }}$ a phase difference $\Delta \varphi=\eta \cdot t_{\text {Gate }}$ was acquired between the rotating frame of the gate operation and the frame of the projection pulse (see Sec. 10.1.2). By adding $\Delta \varphi$ to the projection driving field parameters the designed experiment of an accurate QPT sensing an imperfect quantum gate is realized.

\subsection{Randomized benchmarking}

Based on the protocol introduced by Knill et al. [143] a randomized benchmark (RB) analysis is implemented. The RB investigates the error scaling of concatenated gate sequences realized by a set of Clifford gates. The basic assumption of RB is that for a randomly chosen sequence the noise is depolarizing [16], such that a fully depolarized system is in the completely mixed state $\rho_{m i x}=\frac{1}{d} \sum_{i=1}^{d}\left|\psi_{i}\right\rangle\left\langle\psi_{i}\right|$. By varying the randomized sequence potential dependencies on the selected sequence are averaged out. Measuring the degree of polarization for different sequence length enables a mechanism to study the error scaling for the particular gate modality. Since the $\mathrm{RB}$ applies sets of quantum gates, the average error per gate $\epsilon_{g}$ for the chosen set of gates is extracted, while the error of the individual gates remains undetermined. Among others, randomized benchmarking has been demonstrated utilizing trapped ions [143, 144], liquid NMR [145], superconducting qubits [146] and solid-state qubits [147]. Error probabilities per gate down to $\epsilon_{g}=2.0(2) \cdot 10^{-5}$ have been proven in the physical system of trapped ions [148]. While RB analysis have been demonstrated for multi-qubit gates [145] in the scope of this thesis only single-qubit gates are realized.

\subsubsection{Single qubit randomized benchmarking}

First, $N_{G}$ sequences $\mathcal{G}=\left\{G_{1} \ldots G_{l_{N_{l}}}\right\}$ of randomly generated $\pi / 2$-pulses with a total length $l_{N_{l}}$ are generated. Each sequence is reduced to $N_{l}$ subsequences of length $l_{k}=\left\{l_{1}, l_{2}, \ldots, l_{N_{l}}\right\}$. After calculating the expected measurement outcome of each sequence $\mathcal{G}_{l_{k}}$, a gate $R$ projecting the spin to one of the computational eigenstates $(|0\rangle,|1\rangle)$ is chosen randomly and concatenated to $\mathcal{G}_{l_{k}}$. The resulting sequences $R G_{l_{k}} \ldots G_{1}$ are Pauli randomized $N_{P}$ times. To do so, a random sequence of $\pi$ pulses and identity operations is generated $\mathcal{P}=\left\{P_{1} \ldots P_{l_{k}+2}\right\}$ for every of the $N_{G} \cdot N_{l}$ subsequences. Finally, the $N_{G} \cdot N_{P} \cdot N_{l}$ experimental sequences $\mathcal{S}=\left\{P_{l_{k}+2} R P_{l_{k}+1} G_{l_{k}} \ldots G_{1} P_{1}\right\}$ are concatenated. Since the whole experimental sequence $\mathcal{S}$ is known and the final state $\left|\Psi_{f}\right\rangle$ is designed to be one of the computational eigenstates, an estimate on the sequence fidelity $f$ is obtained by comparing 
the measurement population $\left|\Psi_{\text {meas }}\right\rangle$ with the anticipated one:

$$
f=\left\langle\Psi_{f} \mid \Psi_{\text {meas }}\right\rangle
$$

In case the signal is completely depolarized the measurement of $|0\rangle$ and $|1\rangle$ are equally probable. Therefore, the fidelity $f$ will decay towards 0.5 with increasing sequence length $l_{k}$.

\subsubsection{Experimental implementation}

We generate $N_{G}=4$ random sequences $\mathcal{G}$ of length $l=48$ consisting of $\pi / 2$-pulses representing the computations. Gate operations $G_{k}$ are assumed to be performed by rotations of $\pi / 2$ around a set of orthogonal axis $g=\left\{R_{x}\left(\frac{\pi}{2}\right), R_{y}\left(\frac{\pi}{2}\right), R_{z}\left(\frac{\pi}{2}\right), R_{\bar{x}}\left(\frac{\pi}{2}\right), R_{\bar{y}}\left(\frac{\pi}{2}\right), R_{\bar{z}}\left(\frac{\pi}{2}\right)\right\}$. Each sequence is reduced to $N_{L}=13$ different sequence length $l_{k}=\{2,4,6,8,10,14,18,22,16,30,34,40,48\}$. The expected measurement outcome $\mathcal{M}$ is calculated for the $N_{G} \cdot N_{L}$ sequences. Based on $\mathcal{M}$ the projection pulse $R$ is randomly chosen from a suitable subset of $g$ and added to the respective sequence. The obtained $N_{G} \cdot N_{L}$ sequences are Pauli randomized $N_{P}=8$ times by means of $\pi$-pulses and identity operations $\left(r=\left\{R_{x}(\pi), R_{y}(\pi), R_{z}(\pi), R_{\bar{x}}(\pi), R_{\bar{y}}(\pi), R_{\bar{z}}(\pi), R_{z}(0), R_{z}(2 \pi)\right\}\right)$. In total $N_{G} \cdot N_{L} \cdot N_{P}=416$ different sequences are generated and each of them is averaged $N_{e}=10^{7}$ times.

For the maximal computational sequence length $l_{k}=48$ the total number of gate operations is 99, including the randomization pulses. Therefore, depending on the realized gate concept (see Ch. 12) the maximal total sequence length varies between $t_{\mathrm{HQG}}=37.8 \mu \mathrm{s}, t_{\mathrm{SAGQG}}=31.7 \mu \mathrm{s}$ and $\bar{t}_{\mathrm{DYN}}=17.14 \mu \mathrm{s}$. Due to the different length of the computational gates in the dynamic realization the sequence duration is given as the mean value of the realized $\mathcal{S}$. Decoherence effects can be neglected in good approximation, since the relevant decay time $T_{1 \rho} \approx 5 \mathrm{~ms}$ (Sec. B.1) is much longer than the longest sequence duration.

\subsubsection{Fidelity and error scaling}

The average fidelity $\bar{f}$ of each sequence $\mathcal{S}$ is calculated as described in the following. For each $\mathcal{S}$ the expected measurement outcomes, by design either $|0\rangle$ or $|-\rangle$, are calculated. The population is extracted by detecting the fluorescence level after $\mathcal{S}$ was applied and comparing it to the reference fluorescence level of $|0\rangle$ and $|-\rangle$.

The average fidelity, for each sequence length $l_{k}$, is calculated by averaging over the $N_{G} \cdot N_{P}$ measurements according to [143]:

$$
\bar{f}_{l_{k}}=1-p_{l_{k}}=1-\sum_{j=1}^{N_{G}} \sum_{m=1}^{N_{P}} \frac{p_{j, l_{k}, m}}{N_{G} N_{P}} .
$$

In case of non-systematic errors the result should not differ significantly for each individual sequence $\bar{f}_{j, l_{k}}=1-p_{j, l_{k}}=1-\sum_{m=1}^{N_{P}} \frac{p_{j, l_{k}, m}}{N_{P}}$. Errors correspond to the standard error of the mean $\sigma_{\text {mean }}=$ $\sigma_{\bar{f}_{l_{k}}} / \sqrt{N}$, where $N=N_{P} \cdot N_{G}$ is the number of measurements per sequence length $l_{k}$. A functional of the form [144]

$$
f(l)=1-\left(\left(1-\alpha_{n} \epsilon_{m}\right)\left(1-\alpha_{n} \epsilon_{g}\right)^{l}+1\right) / \alpha_{n}
$$

where $\alpha_{n}=2^{n} /\left(2^{n}-1\right)$ is a factor depending on the number of involved qubits $n$, is fitted to the data extracting the average error per gate $\epsilon_{g}$. The factor $\epsilon_{m}$ combines the average error of the 
initialization, the final projecting $\pi / 2$-pulse, the final Pauli randomization and the readout.

For gate concepts realized on the two-dimensional Hilbert space spanned by $|0\rangle$ and $|-\rangle$ the complete randomized benchmarking analysis can be realized by one gate modality. The HQG realized on three dimensional Hilbert space featuring the computational subspace $|+\rangle,|-\rangle$ requires initialization and readout pulses at the beginning and end of each sequence. Choosing $|-\rangle$ as the initial state requires a $\pi$-pulse on the transition $|0\rangle \leftrightarrow|-\rangle$ at the beginning and end of each measurement. 


\section{Microwave control}

In Ch. 5 the optical components of the experimental apparatus have been discussed. Here, the additional microwave components needed for the coherent manipulation of solid-state spins are introduced. Additionally, details on the generation of driving field sequences and are given.

\subsection{Coherent spin manipulation}

In addition to the optical components of the system, a microwave source is required in order to perform coherent spin manipulation experiments on NV centers (Sec. 2.2). The experimental realization features an arbitrary waveform generator (AWG) (AWG70002A, Tektronix) providing microwave fields in up to two channels at a sampling rate of 25 GSamples/s and with eight bit vertical resolution. Additionally, up to four binary marker output channels are utilized to synchronize the AOMs of the excitation and STED laser, as well as the detection card of the microscope, with the applied microwave fields. Transistor-transistor logic cards controlled by a data acquisition card (PCIe-6353, National instruments) enable the switching between coherent experiment mode and imaging mode. In imaging mode the $\mathrm{AWG}$ is idle and the experiment is fully operated by the control computer (Sec. A.1). A synchronization between AWG and laser pulses is required to allow for time-gated detection in experiment mode. The STED laser was chosen to be the master, delivering the trigger signal to the excitation laser and the AWG. In this way, the STED laser, featuring a trigger output (internal jitter $<2 \mathrm{ps}$ ), is protected against self-damaging caused by failing of the trigger source. At the same time a stable system clock is provided.

The AWG output channel is amplified by a broadband high-power microwave amplifier (ZHL$16 \mathrm{~W}-43+$, Mini-Circuits) and delivered to the sample by low loss SMA-cable (Huber+Suhner). In house designed circuit boards [149] fabricated on low-loss high-frequency circuit material (Rogers Corporation) guide the microwave fields to the sample. A gold micro-wire $(\varnothing=30 \mu \mathrm{m})$ spanned over the diamond sample serves as antenna. Dumping the microwave into an attenuator in combination with a broadband microwave circulator (CS-8, Microwave Communications laboratories, Inc.) prevents back reflections disturbing and potentially damaging the system.

Typically, the in this system obtained Rabi frequencies are in the range of $5-10 \mathrm{MHz}$ for NV centers separated from the gold wire by $50-100 \mu \mathrm{m}$. In order to obtain higher Rabi frequencies $\Omega$-loop antennas might be utilized.

\subsubsection{Sequence creation}

Each coherent spin manipulating experiment consist of three instances, as illustrated in Fig. 10.1.a. First, the quantum system is initialized by illumination with a green laser pulse (Sec. 2.2). Subsequently, the actual experiment is performed by application of microwave driving fields. The AOM double-pass configuration acts as a fast switch turning off the laser beams during this part of the sequence. Finally, the spin state is readout while illumination with another green laser pulse, which at the same time initializes the subsequent experiment. A normalized signal is obtained by 



C
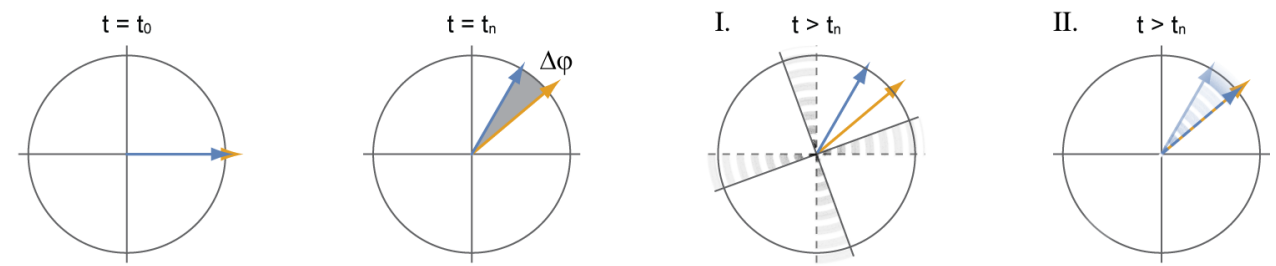

Figure 10.1.: Coherent microwave experiment sequence creation. (a) Illustration of the experimental sequence. A green laser pulse initializes the spin (INI), afterwards the spin manipulation takes place and finally the spin state is read out (RO). By normalising the signal (s.) to a reference (ref.) after reinitialization . (b-c) When switching between two harmonic oscillations of same phase at $t_{0}$ but different frequencies $f_{1}$ (orange line/arrow) and $f_{2}$ (blue line/arrow), at time $t_{n}$ the phase $\Delta \varphi=2 \pi\left(f_{2}-f_{1}\right) t_{n}$ needs to be added in order to maintain the proper rotating frame (blue dashed line/arrow). The additional phase leads to a smooth transition between the two frames rotating with different velocities (II).

comparing the signal fluorescence level (s.) with a reference signal (ref.). The reference is measured for the spin initialized in the $m_{s}=0$ state. Since initialization and readout are identical for all experiments these parts of the sequence are predefined in the control software.

The creation of microwave experiments requires a high degree of flexibility. First the duration of the sequence $t_{\exp }$ is defined. Within $t_{\exp }$ the zero time point $t_{0}$, which represents the reference for all other time points $t_{n}$ of the sequence, is chosen. Note, parts of the experiment might be performed at negative times. According to a list containing the amplitude $A$, the detuning $\Delta$ and the phase $\varphi$ for all time points $t_{n}$ the driving field amplitude $D$ is calculated by:

$$
D\left(t_{n}, \Delta, A, \varphi\right)=A \cos \left(\left(\omega_{0}+2 \pi \cdot \Delta\right) \cdot\left(t_{n}-t_{0}\right)+\varphi\right) .
$$

Obviously, a sinusoidal driving field is obtained for a list containing identical entries for all $A$, $\Delta$ and $\varphi$, respectively. Note, when switching between two driving field frequencies $\omega_{0}+\Delta_{1}$ and $\omega_{0}+\Delta_{2}$ at time point $t_{n}$ a phase jump of $\Delta \varphi=2 \pi t_{n}\left(\Delta_{1}-\Delta_{2}\right)$ occurs (Fig. 10.1.b-c). This phase difference needs to be accounted for if the subsequent part of the experiment is suppose to be performed in the same frame of reference (FoR). Figure 10.1.c illustrates the switching between the FoR. At $t=0$ the two FoRs, represented by the yellow and blue arrows, start at the same position. After time $t_{n}$ the covered angle differs by $\Delta \varphi$. When (I.) switching the driving field frequency without correcting for $\Delta \varphi$ the reference frame is effectively rotated. In contrast, when accounting for $\Delta \varphi$ (II.) the frame of reference remains unchanged.

Note, due to slow drifts of the system resonance frequency $\omega_{0}$ and Rabi frequency $\Omega_{0}$ varies with time. Therefore, an experimental routine retuning the control parameter in preset time intervals has been implemented in order to guarantee measuring at the optimal working point (see Sec. A.2).

\subsubsection{System frequency response}

The advent of arbitrary waveform generators tremendously simplified the generation of timedependent microwave fields and thereby offers high potential in quantum sensing (QS) and QIP. 

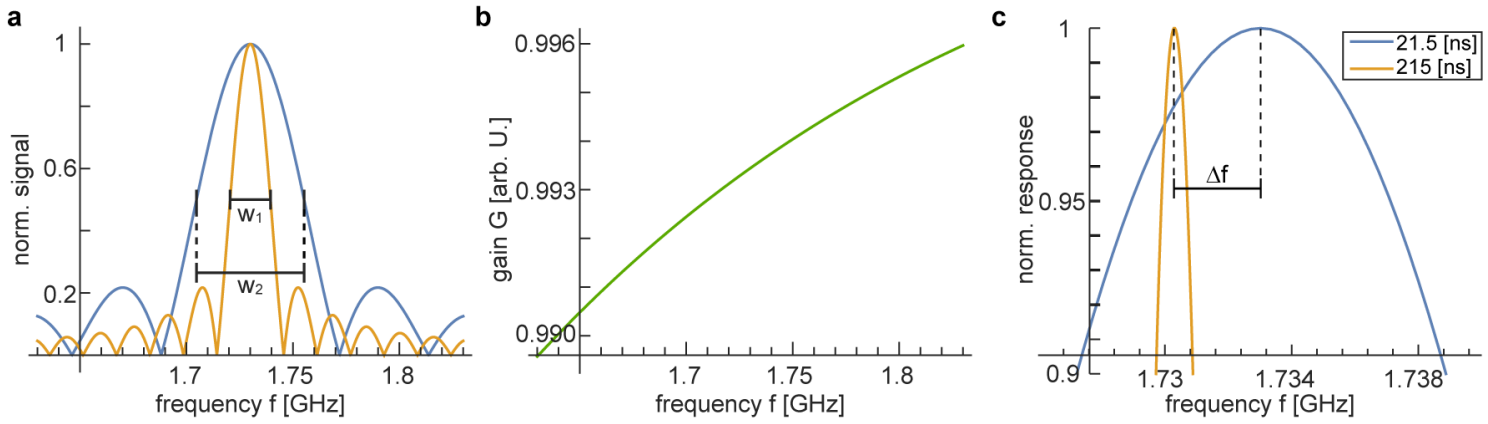

Figure 10.2.: Illustration of the system frequency response. (a) The pulse length (blue 21.5 ns, orange $215 \mathrm{~ns})$ in time-domain determines the width $\left(w_{1}, w_{2}\right)$ of the sinc function in frequency space. (b) A non-homogeneous gain of the microwave amplifier approximated by an error-function (green) weights high frequency components of the sinc function stronger than low frequency components. (c) The weighting causes a shift of the effectively sensed frequency depending on the pulse length in time domain. In the considered case, a tenfold increase of the pulse length causes an relative shift of $\Delta f=800 \mathrm{kHz}$

However, high-power broadband microwave amplifier remain a bottleneck with significant experimental consequences. The microwave amplifier used in the experimental apparatus specifies a uniform gain in the frequency range of $1.8-4.0 \mathrm{GHz}$. As a consequence, at a magnetic field of $|B| \approx 400 \mathrm{G}$, needed for the nuclear polarization of the nitrogen spin (compare B.2), the system is working at or even beyond the limit of the stated frequency range. The involved implications are highlighted in the following and need to be considered in precision measurements. Particularly, in the standard quantum process tomography measurements presented in Sec. 12.5 a frequency shift due to system frequency response is considered.

Consider a $\pi$-pulse described by a cosine of frequency $f_{0}=1.73 \mathrm{GHz}$ multiplied by a rectangular function of duration $d$. The function corresponds to the convolution of a Dirac delta function $\delta\left(f-f_{0}\right)$ with a sinc function $\operatorname{sinc}(f / d)$ (convolution theorem) in frequency domain. Obviously, the width of the sinc in frequency space is depending on the temporal length of the $\pi$-pulse (see Fig. 10.2.a). To describe the signal sensed by the NV center the frequency spectra needs to be multiplied by the frequency depended gain of the experimental system. Since no information on the amplifier gain below $1.8 \mathrm{GHz}$ is available, we utilize an error-function $\operatorname{erf}(f / \sigma)$ with $\sigma=1.8 \mathrm{GHz}$ (Fig. 10.2.b) to illustrate the basic principle. The slope of the error function weights high frequency components of the sinc function stronger than low ones. This results in an effective shift $\Delta f$ of the peak position (Fig. 10.2.c) at position of the defect center. For shorter $\pi$-pulse durations the effect becomes larger due to the increased width of the sinc function in frequency space.

Experimentally, the effect is visualized by taking ESR spectra in the same frequency range with modified $\pi$-pulse length and Rabi frequency (Fig. 10.3.a). Note, a shift of the sensing frequency to higher values causes the resonance within the spectra to appear at lower frequencies. A simulation based on the above presented model reveals a $1 / t_{\pi}$ dependency of the sensing frequency. The experimental resonance frequency, extracted by a Lorentzian fit, depending on the $\pi$-pulse length is fitted by the function $b(t)=$ off $+a / t$ (see Fig. 10.2.b) in good agreement. The effect is reproducible and after once calibrating at a certain frequency, correction factors can be introduced into the experimental routine. Equivalently, the effect is observed for the high transition at $\sim 4.01 \mathrm{GHz}$.

Based on this insight, the strategy to perform precision experiments is the following: First, determine the resonance frequency with a precision, depending on the sample, of up to $\sim 25 \mathrm{kHz}$ by means of a low microwave amplitude ESR measurement. Second, based on a calibration measurement correct the ESR resonance frequency to be on-resonance for full amplitude microwave 
a

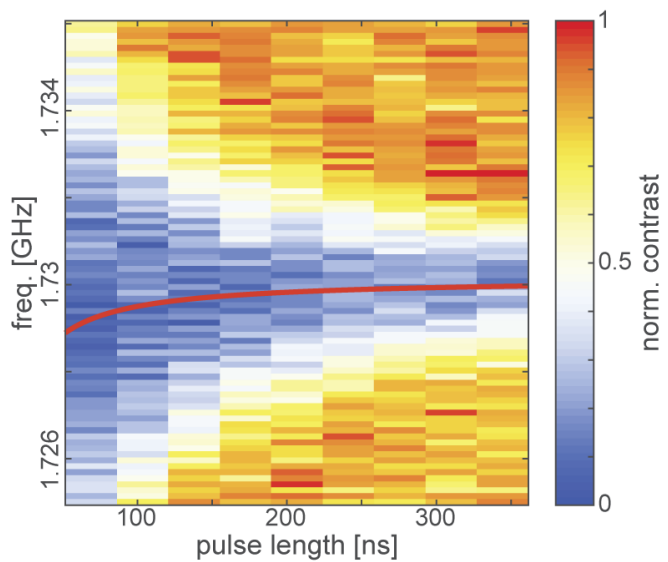

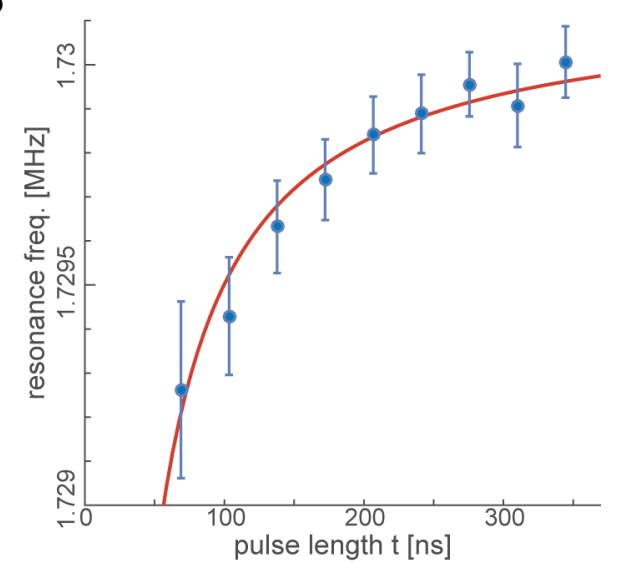

Figure 10.3.: Measured system frequency response. (a) Measured ESR spectra for $\pi$-pulse length between $71 \mathrm{~ns}$ and $355 \mathrm{~ns}$. (b) Resonance frequency extracted by a Lorentzian fit to the spectra in (a). As expected from simulations the experimental data show a $1 / t_{\pi}$ dependency (red line), which is depicted in (a) as well.

experiments. Subsequently, the Rabi oscillation is measured for pulse length where resonant driving is assured. In this way the system is fully characterized and measuring at the optimal working point is guaranteed. 


\section{Superadiabatic geometric quantum gates}

In Ch. 8 the advantages and disadvantages of adiabatic geometric phase based quantum computation were discussed. In summary, the improved robustness of geometric quantum gates comes at the cost of a long gate duration, which makes the system sensitive to open system effects and decoherence effects. Until now, strategies avoiding the need for adiabaticity involved non-Abelian holonomies (see Sec. 8.6) and thereby (at least) three-level systems.

Here, the realization of a recently proposed set of superadiabatic geometric quantum gates [81] based on the concept of transitionless quantum driving [150] is demonstrated. Remarkably, the gates are defined on the two-dimensional Hilbert space (see Sec. 11.3.1). As a consequence, the advantages of non-adiabatic system evolution and geometric phases are combined, while keeping the experimental resources minimal.

We will slowly approach the subject by giving an intuitive example and introducing the fundamental concepts. Afterwards, linearly chirped Gaussian pulses are utilized to realize high-fidelity population transfer and illustrate the advantages of superadiabatic system evolutions. In a proof-ofprinciple realization, the Bloch sphere trajectory of a non-Abelian set of superadiabatic geometric quantum gates is measured. The quality of the gate performance is quantified by determining the quantum gate fidelity by standard quantum process tomography. Finally, the robustness at the boarder of the accessible parameter space is investigated.

\subsection{Superadiabatic geometric quantum driving}

Superadiabatic geometric quantum driving provides a means to accelerate an adiabatic geometric process without disturbing its energy eigenstates. The intuitive example in Sec. 11.1.1 illustrates how a local rotation can suppress transitions between the energy eigenstates, as a requirement for purely geometric evolution, of a non-adiabatically driven system. Accelerated driving field frames of reference as a tool for transitionless quantum driving are discussed in Sec. 11.1.2. Finally, in Sec. 11.1.3 a general Hamiltonian for superadiabatic, transitionless quantum driving is derived.

\subsubsection{Intuitive explanation}

Based on the work of Sels et al. [151], we start with presenting an intuitive illustration of the basic concept of superadiabatic quantum driving in a classical context. Imagine a waiter serving a full glass of water placed on a plate. When performing the transport adiabatically, the glass will arrive at the table without any excitation spilling the water (Fig. 11.1.a). However, the waiter needs to perform the transport as fast as possible in order to maximize his tip. Accelerating the transport on the same trajectory causes a pseudo force in the frame of reference of the tablet. This pseudo force excites the water in the glass and causes it to spill over (b). By appropriately rotating the tablet the pseudo force can be countered (c), finally enabling a fast and robust transport. For this 
a

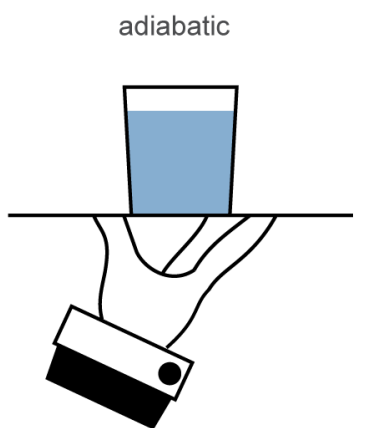

b

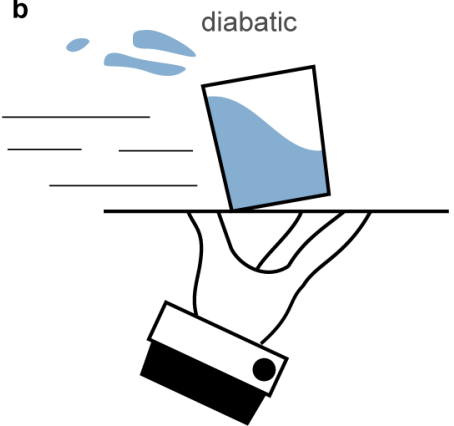

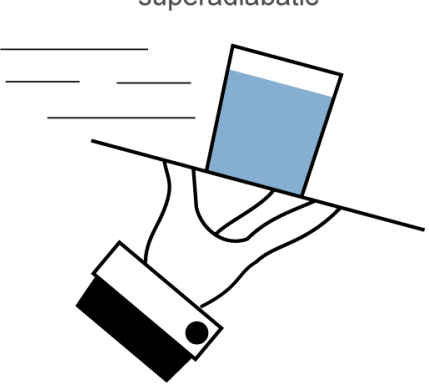

Figure 11.1.: Intuitive introduction of the superadiabatic concept. (a) When transporting a glass of water adiabatically the water on the tablet remains unexcited. (b) Performing the transport along the same path in an accelerated manner the arising pseudo force excites the water in the glass, causing the water to spill over. (c) By precisely rotating the plate the pseudo force might be countered completely, achieving a controlled and fast transport.

reason the superadiabatic approach is sometimes referred to as counter-diabatic [152]. Following the nomenclature of the original work by M.V. Berry and R. Lim introducing the superadiabatic basis [153] we stick with the term superadiabatic in the following.

Let us now transfer this analogy into the quantum world. An adiabatically changing original Hamiltonian $H_{0}(t)$ drives the instantaneous eigenstates $\left|\lambda_{ \pm}(t)\right\rangle$ without transitions between them (parallel transport). Here, the instantaneous energy eigenstates are the equivalent to the level of excitation of the water within the glass. Transitions between the instantaneous eigenstates occur when the evolution is accelerated. To be accurate, also for a adiabatically slow driven Hamiltonian the transition amplitudes of the instantaneous eigenstates are exponentially weak but non-zero. In order to prevent transitions between the eigenstates a correction Hamiltonian $H_{C}(t)$ can be derived, which is countering the arising "pseudo-forces" [150]. The sum of $H_{0}(t)$ and $H_{C}(t)$ defines the superadiabatic Hamiltonian

$$
\hat{H}(t)=H_{0}(t)+H_{C}(t)
$$

driving the original Hamiltonian arbitrary fast. In fact, the concept of transitionless quantum driving (TQD) finds a neighbouring Hamiltonian $\hat{H}$ which sets the transition amplitudes to precisely zero independently of the degree of slowness [150]. Since $H_{1}(t)$ incorporates a time-dependent phase, which might be transformed into a time-dependent detuning (e.g., [154]), the frame of reference (FoR) is rotated similarly to the tablet of the waiter. Already in classical mechanics time-dependently accelerated FoR are non-trivial problems and we briefly discuss accelerated FoR in the quantum mechanical context within the next section.

\subsubsection{Accelerated driving field frames}

Following the proposal by Liang et al. the superadiabatic geometric quantum gate [81] is defined on the two dimensional Hilbert space and is driven time dependently. Consequentially, the Hamiltonian in the laboratory frame $H_{L}(t)$ follows as the one of a two-level system with energy spacing 
$\hbar \omega_{0}$ and a driving field of angular frequency $\omega_{\mathrm{D}}(t)$, phase $\varphi$ and amplitude $\Omega_{\mathrm{R}}(t)$ :

$$
\begin{aligned}
H_{L}(t) & =\frac{\hbar}{2}\left(\begin{array}{cc}
\omega_{0} & 2 \Omega_{\mathrm{R}}(t) \cos \left(\omega_{\mathrm{D}}(t) t+\varphi\right) \\
2 \Omega_{\mathrm{R}}(t) \cos \left(\omega_{\mathrm{D}}(t) t+\varphi\right) & -\omega_{0}
\end{array}\right) \\
& =\frac{\hbar}{2}\left(\begin{array}{cc}
\omega_{0} & \Omega_{\mathrm{R}}(t)\left(e^{i\left(\omega_{\mathrm{D}}(t) t+\varphi\right)}+e^{-i\left(\omega_{\mathrm{D}}(t) t+\varphi\right)}\right) \\
\Omega_{\mathrm{R}}(t)\left(e^{i\left(\omega_{\mathrm{D}}(t) t+\varphi\right)}+e^{-i\left(\omega_{\mathrm{D}}(t) t+\varphi\right)}\right) & -\omega_{0}
\end{array}\right) .
\end{aligned}
$$

In order to move into the FoR rotating with the driving field, the transformation $\widetilde{H}=U H_{L} U^{\dagger}+$ $i \hbar \frac{\partial U}{\partial t} U^{\dagger}$ is performed. Here, $U$ is the unitary matrix $U=e^{-i / \hbar H_{D}(t) t}$ incorporating the transformation matrix:

$$
H_{D}(t)=\frac{\hbar}{2}\left(\begin{array}{cc}
\omega_{\mathrm{D}}(t) & 0 \\
0 & -\omega_{\mathrm{D}}(t)
\end{array}\right)
$$

In contrast to driving fields of fixed frequency, the explicit time dependence of $\omega_{\mathrm{D}}(t)$ causes the second term $\left(i \hbar \frac{\partial U}{\partial t} U^{\dagger}\right)$ to contain two contributions $\left(\omega_{D}(t)\right.$ and $\left.\dot{\omega}_{D}(t) t\right)$. Performing the transformation explicitly leads to

$$
\begin{aligned}
\widetilde{H}(t) & =\frac{\hbar}{2}\left(\begin{array}{cc}
\Delta(t)+\dot{\Delta}(t) t & \Omega_{\mathrm{R}} e^{i \omega_{\mathrm{D}}(t) t}\left(e^{i\left(\omega_{\mathrm{D}}(t) t+\varphi\right)}+e^{-i\left(\omega_{\mathrm{D}}(t) t+\varphi\right)}\right) \\
\Omega_{\mathrm{R}} e^{-i \omega_{\mathrm{D}}(t) t}\left(e^{i\left(\omega_{\mathrm{D}}(t) t+\varphi\right)}+e^{-i\left(\omega_{\mathrm{D}}(t) t+\varphi\right)}\right) & -(\Delta(t)+\dot{\Delta}(t) t)
\end{array}\right) \\
& =\frac{\hbar}{2}\left(\begin{array}{cc}
\Delta(t)+\dot{\Delta}(t) t & \Omega_{\mathrm{R}}\left(e^{i\left(2 \omega_{\mathrm{D}}(t) t+\varphi\right)}+e^{-i \varphi}\right) \\
\Omega_{\mathrm{R}}\left(e^{i \varphi}+e^{-i\left(2 \omega_{\mathrm{D}}(t) t+\varphi\right)}\right) & -(\Delta(t)+\dot{\Delta}(t) t)
\end{array}\right) \\
& =\frac{\hbar}{2}\left(\begin{array}{cc}
\Delta(t)+\dot{\Delta}(t) t & \Omega_{\mathrm{R}} e^{i \varphi} \\
\Omega_{\mathrm{R}} e^{-i \varphi} & -(\Delta(t)+\dot{\Delta}(t) t)
\end{array}\right),
\end{aligned}
$$

where $\Delta(t)=\omega_{0}-\omega_{\mathrm{D}}(t)$ is the detuning from resonance and $\dot{\Delta}(t)=\dot{\omega}_{D}(t)$ its temporal derivative. In the last step the rotating wave approximation (RWA), cancelling out high frequency components, was performed. A similar Hamiltonian might be derived to describe processes in the $d$-dimensional Hilbert space. Note, the temporal change of the driving field frequency $\omega_{D}(t)$ results in an effective acceleration of the driving field frame $(\Delta(t)+\dot{\Delta}(t) t)$. However, the proposal by Liang et al. [81] performs the derivation of the superadiabatic Hamiltonian for the effective detuning:

$$
\Delta_{\text {eff }}(t)=\Delta(t)+\dot{\Delta}(t) t
$$

In order to obtain the driving field frequency $\omega_{\mathrm{D}}(t)=\omega_{0}+\Delta(t)$ in the laboratory frame the above differential equation needs to be solved whenever $\Delta_{\text {eff }}(t)$ is given. When designing $\Delta_{\text {eff }}(t)$ it needs to be ensured that at least one analytic or numeric solution exists.

\subsubsection{Superadiabatic Hamiltonian}

In Sec. 11.1.2 a general expression for the Hamiltonian of a time dependently driven two-level system was derived (Eq. 11.4). Due to its deviation to the one utilized by Liang et al. in their proposal of the superadiabatic quantum gates [81] a validation of the proposal is required. Starting from Eq. 11.4 we perform the derivation explicitly (App. C). In essence, the in [81] derived 
superadiabatic Hamiltonian $H_{S}(t)$ holds true when replacing the effective detuning $\Delta_{\text {eff }}(t)$ by $\Delta(t)+\dot{\Delta}(t) t$ in all terms, such that follows:

$$
H_{S}(t)=\frac{\hbar}{2}\left(\begin{array}{cc}
\Delta(t)+\dot{\Delta}(t) t & \Omega_{\mathrm{S}}(t) e^{-i\left[\varphi+\psi_{\mathrm{S}}(t)\right]} \\
\Omega_{\mathrm{S}}(t) e^{i\left[\varphi+\psi_{\mathrm{S}}(t)\right]} & -(\Delta(t)+\dot{\Delta}(t) t)
\end{array}\right) .
$$

Here the superadiabatic Rabi-frequency

$$
\Omega_{\mathrm{S}}(t)=\sqrt{\Omega_{\mathrm{R}}^{2}(t)+\Omega_{\mathrm{C}}^{2}(t)}
$$

and the superadiabatic phase

$$
\psi_{\mathrm{S}}(t)=\arctan \left[\Omega_{\mathrm{C}}(t) / \Omega_{\mathrm{R}}(t)\right]
$$

are defined. Both parameters vary over time and depend on the corrected Rabi frequency

$$
\Omega_{\mathrm{C}}(t) \equiv \dot{\theta}=\frac{\left[\dot{\Omega}_{\mathrm{R}}(t)(\Delta(t)+\dot{\Delta}(t) t)-\Omega_{\mathrm{R}}(t) \frac{\partial}{\partial t}(\Delta(t)+\dot{\Delta}(t) t)\right]}{\Omega(t)^{2}}
$$

which incorporates the generalized Rabi frequency $\Omega(t)=\sqrt{\Omega_{\mathrm{R}}(t)^{2}+(\Delta(t)+\dot{\Delta}(t) t)^{2}}$.

\subsection{Population transfer by a linearly chirped Gaussian pulse}

The general expression in Eq. 11.6 represents a tool to speed up time dependent adiabatic processes in a two-level system. First experimental demonstrations performed the population transfer of a Bose-Einstein condensate by the standard Landau-Zener [155] and tangent protocols [155, 156] superadiabatically. Recently, TQD has demonstrated the population transfer of solid statespins by optical stimulated Raman adiabatic passage (STIRAP) in a three-level system [157, 158]. While these demonstrations resulted in a precise control of the population, the phase remained uncontrolled. Thereby prohibiting their use for quantum information processing. In the following we will focus on two-level systems in order to keep the experimental resources minimal.

Recently, Dou et al. [154] proposed to apply the concept of TQD to a linearly chirped Gaussian pulse (LCGP) in order to perform a fast and robust population inversion of the targeted quantum system (Landau-Zener problem [159]). Even though, the superadiabatic schemes were already utilized to accelerate the adiabatic population inversion by transporting through an avoided level anti-crossing [155], the proposal is realized in order to illustrate the general concept of superadiabatic quantum driving and highlight its advantages.

The original Hamiltonian features a linear sweep of the detuning $\Delta_{\text {eff }}(t)=2 \cdot \Delta(t)=2 \alpha t$ (Fig. 11.2.b), while the Rabi frequency $\Omega(t)=\Omega_{0} \exp \left(-t^{2} / T^{2}\right)$ exhibits a Gaussian envelope centered around $t=0$ (a). In our experiments we set the constants $\alpha=27.4 \mathrm{kHz} \mathrm{ns}^{-1}, \Omega_{0}=7 \mathrm{MHz}$ and $T=35.7 \mathrm{~ns}$. When adding the correction Hamiltonian $H_{C}(t)$ in order to obtain a superadiabatic system evolution the detuning $\Delta(t)$ remains unchanged, while the Rabi frequency function $\Omega_{\mathrm{S}}(t)$ (orange) receives additional side lobes (a). Additionally, the time-dependent superadiabatic phase $\psi_{\mathrm{S}}(t)$ defined by Eq. 11.8 occurs (c). Theoretically, in order to reduce the number of control parameters, an appropriate transformation can incorporate the effect of the superadiabatic phase $\psi_{\mathrm{S}}(t)$ in the detuning term. This results in an effective detuning $\Delta_{\text {eff }}(t)=2 \Delta(t)+\dot{\phi}_{s}(t) / 2(\mathrm{~b}$, orange) and a vanishing phase $\varphi(t)=0$. However, standardized evaluation routines fail to solve 



Figure 11.2.: Superadiabatic population transfer by linearly chirped Gaussian pulses. (a) Original $\Omega(t)$ (blue) and superadiabatic $\Omega_{\mathrm{S}}(t)$ (orange) Rabi frequency. (b) Original $\Delta(t)$ (blue) and effective $\Delta_{\text {eff }}(t)$ (orange) detuning. (c) Superadiabatic phase $\psi_{\mathrm{S}}(t)$. (d) Experimentally measured (dots) and calculated (lines) population obtained by a Rabi experiment (blue), a linearly chirped Gaussian (green) and a superadiabatic linearly chirped Gaussian pulses (orange).

the differential equation for $\Delta_{\text {eff }}(t)=\Delta(t)+\dot{\Delta}(t) t$ and experiments are performed in the rotating frame of the original driving field.

In Fig. 11.2.d the population as a function of time is presented for a Rabi oscillation (orange), an adiabatic LCGP (green) and a superadiabatic LCGP (blue), respectively. The Rabi experiment leads to the fastest inversion of the population after $t_{\pi}=\pi / \Omega_{0} \approx 71 \mathrm{~ns}$. However, in order to obtain a close to perfect inversion efficiency strict timing requirements need to be fulfilled. The original adiabatic LCGP Hamiltonian fails to invert the population since the adiabatic condition $t \gg 2 \pi / \Omega_{0}$ is not fulfilled. In contrast, the superadiabatic realization leads to a perfect state inversion within a finite time interval and without strict timing conditions. The subsequent application of several pulses leads to fast loss of coherence for adiabatic LCGP, even though the difference appears to be small.

\subsection{Non-commuting superadiabatic single-qubit quantum gates}

Universal quantum computation requires a set of at least two non-Abelian single-qubit gates as well as one non-trivial two-qubit gate. Non-Abelian holonomies are know to arise in a three or more level system [124], while in general two-level systems are restricted to Abelian operations. In their proposal [81] Liang et al. utilize the approach of S.-L. Zhang and Z.D. Wu to obtain non-Abelian operation in a two-level system by controlling the evolution of a pair of orthogonal cyclic states [160, $161,162]$. In essence, two non-commuting quantum gates are obtained by realizing the phase shift gate in different bases. Let $|k\rangle, k=0,1$ and $| \pm\rangle=\frac{1}{\sqrt{2}}(|0\rangle \pm|1\rangle)$ be two sets of orthogonal states and define the phase-shift gates $|k\rangle \rightarrow \exp [i(2 k-1) \gamma]|k\rangle$ and $| \pm\rangle \rightarrow \exp \left[ \pm i \gamma^{\prime}\right]| \pm\rangle$. All possible single-qubit gates in the computational space spanned by $|0\rangle$ and $|1\rangle$ can be realized by combining these two phase-gates. 


\subsubsection{Pair of orthogonal cyclic states}

Here, the time-evolution operator $U$ realizing non-Abelian, geometric, single-qubit quantum gates for a pair of orthogonal cyclic states is described. Consider the instantaneous eigenstates of the Hamiltonian in Eq. 11.6:

$$
\left|\lambda_{+}\right\rangle=\left(\begin{array}{c}
\cos \frac{\theta}{2} e^{-i \varphi / 2} \\
\sin \frac{\theta}{2} e^{i \varphi / 2}
\end{array}\right), \quad\left|\lambda_{-}\right\rangle=\left(\begin{array}{c}
-\sin \frac{\theta}{2} e^{-i \varphi / 2} \\
\cos \frac{\theta}{2} e^{i \varphi / 2}
\end{array}\right)
$$

where $\theta=\arctan \left(\Omega_{\mathrm{S}}(t) /(\Delta(t)+\dot{\Delta}(t) \cdot t)\right)$. In fact, every pair of orthogonal states may be parametrized by the above expression. All possible input states can be expressed as a superposition of the eigenstates according to $\left|\psi_{i}\right\rangle=a_{+}\left|\lambda_{+}(0)\right\rangle+a_{-}\left|\lambda_{-}(0)\right\rangle$. Obviously, $a_{ \pm}$can be calculated according to $a_{ \pm}=\left\langle\lambda_{ \pm}(0) \mid \psi_{i}\right\rangle$ and need to be well normalized $\left|a_{+}\right|^{2}+\left|a_{-}\right|^{2}=1$. Assume a cyclic evolution of the system $\left|\lambda_{ \pm}(T)\right\rangle=\exp \left(i \phi_{ \pm}\right)\left|\lambda_{ \pm}(0)\right\rangle[161]$, with real valued phase factor $\phi_{ \pm}$. If a path in parameter space is chosen such that dynamic contributions cancel out, pure geometric evolution $U(T)\left|\lambda_{ \pm}(0)\right\rangle=\exp ( \pm i \gamma)\left|\lambda_{ \pm}(0)\right\rangle$ is obtained. By solving this equation for $U$, under the assumption $\chi=\theta(0)=\{2 \pi n, n \in \mathbb{Z}\}$ or $\chi=\left\{2 \pi n-\frac{3 \pi}{2}, n \in \mathbb{Z}\right\}$, the unitary time-evolution operator $U(T)$ is determined to be (Sec. C.2)

$$
U(\chi, \gamma)=\left(\begin{array}{cc}
\cos \gamma+i \cos \chi \sin \gamma & i \sin \chi \sin \gamma e^{-i \varphi} \\
i \sin \chi \sin \gamma e^{i \varphi} & \cos \gamma-i \cos \chi \sin \gamma
\end{array}\right)
$$

with $\chi=\theta(0)$ being the mixing angle at $t=0$ [160]. From $U\left(\chi_{1}, \gamma_{1}\right) U\left(\chi_{2}, \gamma_{2}\right)=U\left(\chi_{2}, \gamma_{2}\right) U\left(\chi_{1}, \gamma_{1}\right)$ follows immediately that two gates are only commuting when $\sin \left(\gamma_{1}\right) \sin \left(\gamma_{2}\right) \sin \left(\chi_{1}-\chi_{2}\right)=0$. This corresponds to the cases where at least one of the gates is the identity operation $\left(\gamma_{1} \vee \gamma_{2} \bmod \pi=0\right)$ or both rotation are performed around the same axis $\left(\left[\chi_{1}-\chi_{2}\right] \bmod \pi=0\right)$. Setting $\chi=\pi / 2$ enables rotations around arbitrary unit vectors in the plain spanned by $\hat{x}$ and $\hat{y}$. Particularly, rotation around the $\mathrm{x}$ and $\mathrm{y}$-axis are obtained for $\varphi=0$ and $\varphi=\pi / 2$, respectively. For $\chi=0$ the phase shift gate $U=\exp \left(i \gamma \sigma_{z}\right)$ is realized, independently of $\varphi$.

\subsubsection{Proposed realization}

In order to realize a SAGQG the adiabatic "orange slice"-like trajectory of the instantaneous eigenstates is realized to generate a geometric phase equivalent to half the enclosed solid angle $\gamma=\widetilde{\Omega} / 2$. Figure 11.3.a illustrates the anticipated Bloch sphere trajectory along A-B-C-D-A generating a Pauli-Z gate in four steps, each of length $\tau$. The trajectory can be realized adiabatically by applying the time-dependent Rabi frequency [81]

$$
\Omega_{\mathrm{R}}(t)=\left\{\begin{array}{cc}
\Omega_{0}\left[1-\cos \left(\frac{\pi t}{\tau}\right)\right], & 0 \leq t<\tau \\
\Omega_{0}\left[1+\cos \left(\frac{\pi(t-\tau}{\tau}\right)\right], & \tau \leq t<2 \tau \\
\Omega_{0}\left[1-\cos \left(\frac{\pi(t-2 \tau)}{\tau}\right)\right], & 2 \tau \leq t<3 \tau \\
\Omega_{0}\left[1+\cos \left(\frac{\pi(t-3 \tau)}{\tau}\right)\right], & 3 \tau \leq t \leq 4 \tau
\end{array}\right.
$$


a

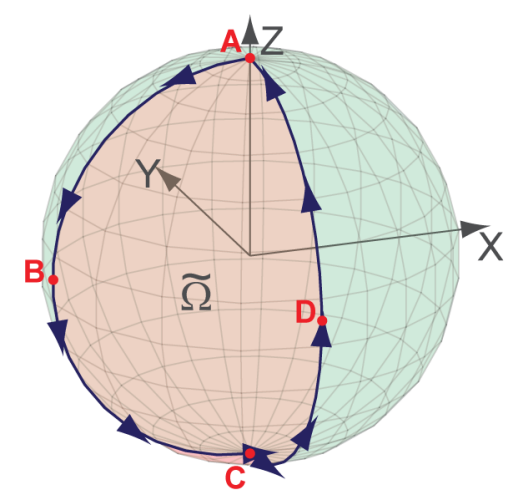

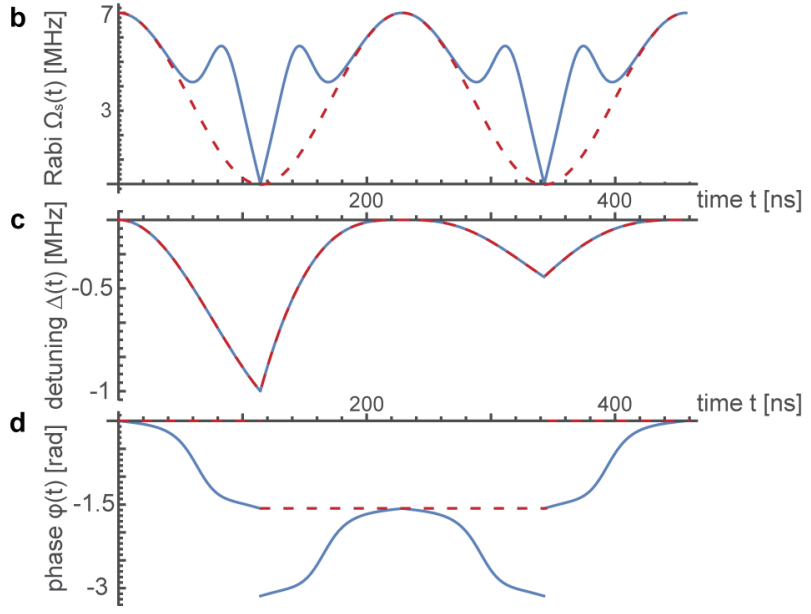

Figure 11.3.: Anticipated trajectory and driving field parameter. (a) Anticipated cyclic evolution of the instantaneous eigenstate along the path A-B-C-D-A. Original (red dashed line) and superadiabatic (solid blue line) (b) Rabi frequency, (c) detuning and (d) phase driving the evolution shown in (a) as a function of time $t$.

and detuning

$$
\Delta_{\mathrm{eff}}(t)=\Delta(t)+\dot{\Delta}(t) t=\left\{\begin{array}{cc}
\Delta_{0}\left[\cos \left(\frac{\pi t}{\tau}\right)+1\right], & 0 \leq t<\tau \\
\Delta_{0}\left[\cos \left(\frac{\pi(t-\tau}{\tau}\right)-1\right], & \tau \leq t<2 \tau \\
\Delta_{0}\left[\cos \left(\frac{\pi(t-2 \tau)}{\tau}\right)+1\right], & 2 \tau \leq t<3 \tau \\
\Delta_{0}\left[\cos \left(\frac{\pi(t-3 \tau)}{\tau}\right)-1\right], & 3 \tau \leq t \leq 4 \tau
\end{array} .\right.
$$

From Eq. 11.11 and Eq. 11.12 follows that $\chi=\theta(0)=0$ as required for the realization of a Pauli-Z gate (see Sec. 11.3.1). The enclosed solid angle $\widetilde{\Omega}=2 \gamma=2\left(\pi-\left(\varphi_{2}^{\prime}-\varphi_{1}^{\prime}\right)\right)$ is determined by the phase factors $\varphi_{1}^{\prime}$ and $\varphi_{2}^{\prime}$ applied according to:

$$
\varphi(t)=\left\{\begin{array}{ll}
\varphi_{1}^{\prime}, & 0 \leq t<2 \tau \\
\varphi_{2}^{\prime}, & 2 \tau \leq t<4 \tau
\end{array} .\right.
$$

Similar time-dependent driving fields have already been used to demonstrate adiabatic geometric quantum computation [163]. From this original Hamiltonian we obtain the superadiabatic geometric driving parameter by calculating $\Omega_{\mathrm{S}}(t)$ according to Eq. 11.6 and solving the differential equation $\Delta_{\text {eff }}(t)=\Delta(t)+\dot{\Delta}(t) t$ for $\Delta(t)$. The driving field parameter of the original (red) and superadiabatic (blue) Hamiltonian are shown in Fig. 11.3.b-d for $\Omega_{0}=3.5 \mathrm{MHz}, \Delta_{0}=1 \mathrm{MHz}$ and $\tau=114 \mathrm{~ns}$. For the realization of a Pauli-Z gate the required value of $\gamma=\pi / 2$ is obtained by setting $\varphi_{1}^{\prime}=0$ and $\varphi_{2}^{\prime}=\pi / 2$.

In Sec. 8.4 the two-loop approach cancelling out the dynamic contribution of the system evolution was discussed. Calculating $\phi_{d y n}(t)=-\frac{1}{\hbar} \int\langle\Psi(t)|H(t)| \Psi(t)\rangle \mathrm{d} t$ reveals the rise of a dynamic phase during the gate evolution. The purely geometric phase at the end of the gate sequence is obtained by the two loop approach resulting in a self-compensating trajectory.

Analogously, the driving field parameter for the Pauli-X gate follow for the trajectory B-C-DA-B. Therefore, the parameter $\Delta_{\text {eff }}(t), \Omega_{\mathrm{R}}(t)$ and $\psi_{\mathrm{S}}(t)$ are precisely the ones of the Pauli-Z gate except for a time shift of $\tau$. Major difference only occur for the applied detuning $\Delta(t)$ which again 
is calculated by solving the differential equation Eq. 11.7. Effectively, for the same parameter set $\Delta_{0}, \Omega_{0}$ and $\tau$ the maximal applied detuning is twice as high as the one of the Pauli-Z gate $\left(\max _{t} \Delta_{x}(t)=2 \times \max _{t} \Delta_{z}(t)\right)$. While this difference from the theoretical point of view does not influence the superadiabatic Hamiltonian, experimental implications like an insufficient driving might occur for large detuning values $\Delta_{0}$.

\subsection{Parameter optimization}

In Sec. 11.3.2 the time dependent expressions for the original Hamiltonians amplitude $\Omega_{\mathrm{R}}(t)$, detuning $\Delta(t)$ and phase $\varphi(t)$ realizing superadiabatic geometric quantum computation are given. The expressions depend on the three free parameters $\Omega_{0}, \Delta_{0}$ and $\tau$ without strict theoretical confinements. However, the experimental apparatus sets boundary conditions in terms of, e.g., the maximal applicable Rabi frequency $\Omega_{\max }(\approx 7.5 \mathrm{MHz}$ in our realization). Due to the appearance of $\Delta(t)$ in Eq. 11.9 describing the superadiabatic Rabi frequency, all free parameters contribute to the final shape of driving field amplitude $\Omega_{\mathrm{S}}(t)$. In the adiabatic limit $\tau \gg 2 \pi / \Omega_{\max }$ one finds that $\Omega_{\mathrm{C}}(t) \rightarrow 0$ and therefore, $\Omega_{\mathrm{S}}(t) \rightarrow \Omega_{\mathrm{R}}(t)$ (solid blue line Fig. 11.4.a). In the regime $\tau \approx 2 \pi / \Omega_{\max }$ the contribution of $\Omega_{\mathrm{C}}(t)$ to $\Omega_{\mathrm{S}}(t)$ becomes pronounced. In general, $\Omega_{\mathrm{C}}(t)$ (dashed lines) adds side lobes to $\Omega_{\mathrm{S}}(t)$ (solid lines), which increase for small $\tau$ and $\Omega_{0}$ values (indicated by red arrow). Intuitively fast gate operation requires strong $\Omega_{\mathrm{C}}(t)$ contributions, which eventually cause $\Omega_{\mathrm{S}}(t)$ to exceed the maximally allowed value of $\Omega_{\max }$ (solid orange line). This results in an insufficient driving of the Hamiltonian (shaded yellow area). The available coherence time is exploited best for the parameter set $\left\{\Omega_{0}, \Delta_{0}\right\}$ which enables the minimization of $\tau$ without exceeding any experimental limitation. Since no analytical expression connecting the maximal amplitude of $\Omega_{\mathrm{S}}(t)$ to the free parameter $\Delta_{0}, \Omega_{0}$ and $\tau$ could be obtained, a numerical analysis was performed. The analysis calculates the minimal $\tau$-value for a set of $\Delta_{0}$ and $\Omega_{0}$ fulfilling the confinements

$$
\tau_{\min }\left(\Omega_{0}, \Delta_{0}\right)=\left(2 / \max _{t}\left(\Omega_{\mathrm{S}}\left(t, \Omega_{0}, \Delta_{0}\right)\right)\right) \wedge \Omega_{\mathrm{S}}(t) \leq \Omega_{\max } \quad \forall t .
$$

Note, $\tau_{\min }$ is a consequence of the specific experimental limitations rather than a theoretical constraints due to, e.g., the need for adiabaticity. The contour plot in Fig. 11.4.b presents the results of the analysis for the Pauli-X gate. Since $\Omega_{\mathrm{S}}(t)$ is equivalent for rotations by arbitrary angles $\varphi$ around the $\mathrm{x}\left(R_{x}(\varphi)\right), \mathrm{y}\left(R_{y}(\varphi)\right)$ and z-axis $\left(R_{z}(\varphi)\right)$ up to a time shift of $\tau$, the presented analysis is valid for every SAGQG realization on the employed experimental apparatus. The parameter $\Omega_{0}$ is limited to $\leq 3.5 \mathrm{MHz}$ due to a factor of 2 in Eq. 11.11 for $t=\tau, 3 \tau$. There are no theoretical restrictions on the value of $\Delta_{0}$ but experimentally sufficient driving is guaranteed for detunings in the order of the power broadened linewidth. A global minimum $\min _{\Omega_{0}, \Delta_{0}}\left(\tau_{\min }\right) \approx 71 \mathrm{~ns}=t_{\pi}$ is located in the parameter region around $\Omega_{0}=1.5 \mathrm{MHz}$ and $\Delta_{0}=1.5 \mathrm{MHz}$. In conclusion, the minimal practical achievable SAGQG gate duration $t_{\text {Gate }}=4 t_{\pi}=4 \pi / \Omega_{\max }$ is four times the length of a dynamic $\pi$-pulse at maximal Rabi frequency $\Omega_{\max }$. Utilizing a highly suitable diamond sample and the above parameter set, roughly 10.000 gate realizations can be performed within the available longitudinal relaxation time $T_{1 \rho}=5.05 \pm 1.56 \mathrm{~ms}$ (Sec. B.1).

For first proof-of-principle realizations (Sec. 11.5 and Sec. 11.6) the parameter set $\Omega_{0}=3.5 \mathrm{MHz}$, $\Delta_{0}=1 \mathrm{MHz}$ and $\tau=0.8 / t_{\pi} \approx 114 \mathrm{~ns}$ was chosen in order to ensure stable experimental conditions. In Sec. 11.8 the derived practical parameter limits are investigated by varying the $\tau$-value around the boundary value $\tau_{\min }$. 

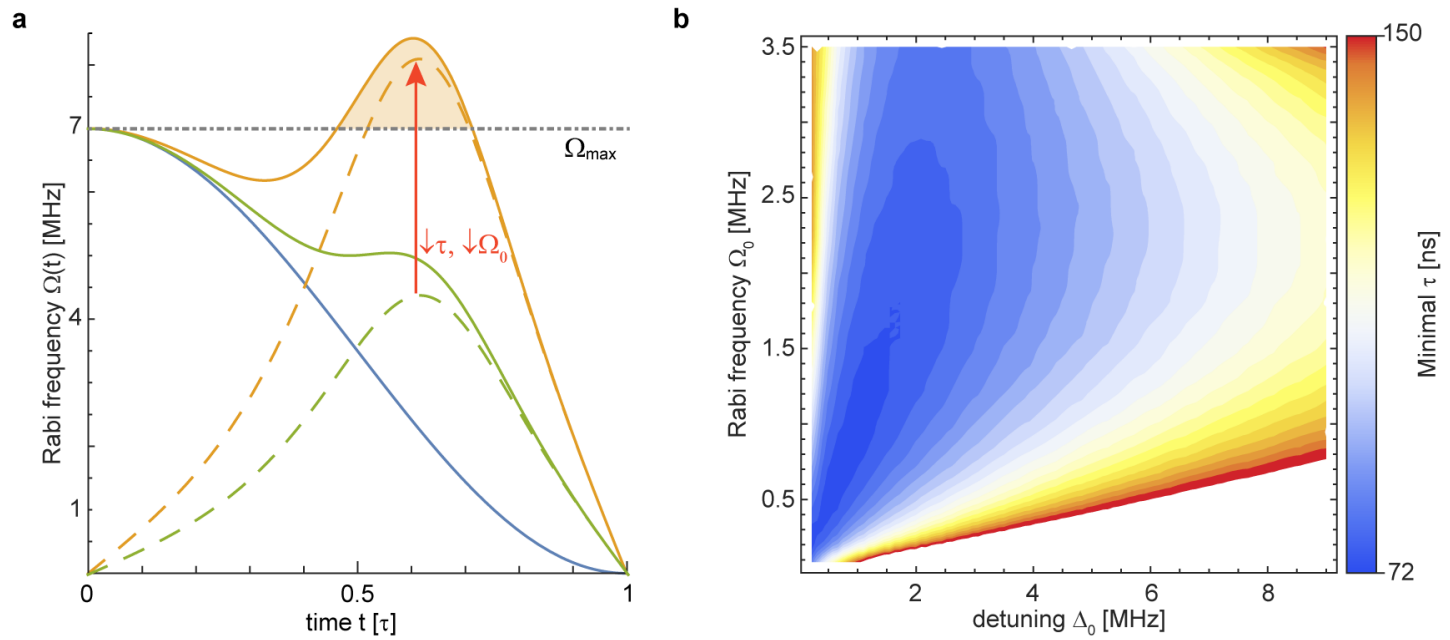

Figure 11.4.: Superadiabatic quantum gate parameter optimization. (a) Original Rabi frequency $\Omega_{\mathrm{R}}(t)$ (blue), correction Rabi frequency (dashed lines) and superadiabatic Rabi frequency (solid lines) for $\Delta_{0}=2 \mathrm{MHz}, \Omega_{0}=2 \mathrm{MHz}$ and $\tau=120 \mathrm{~ns}$ green $(\tau=60 \mathrm{~ns}$ orange). The black dashed line represents the threshold equivalent to maximal applicable driving field strength $\Omega_{\max }$. Rabi frequencies above the threshold (shaded orange area) can not be driven sufficiently. The red arrow indicates a increase of the side lobe amplitude with decreasing $\tau$ and $\Omega_{0}$. (b) Minimal gate parameter $\tau_{\text {min }}$ depending on the detuning $\Delta_{0}$ and Rabi frequency $\Omega_{0}$ fulfilling the requirement $\Omega_{\mathrm{S}}\left(t, \tau, \Delta_{0}, \Omega_{0}\right) \leq \Omega_{\max }$ at all times.

\subsection{Bloch-sphere trajectory}

After theoretically investigating the accessible parameter space in Sec. 11.4, here the SAGQG is demonstrated in a proof-of-concept realization. The Bloch sphere trajectory during the superadiabatic gate operation is measured in a stroboscopic manner. The QG is run for a time $t_{i}$ and subsequently the Bloch sphere coordinates $\left(\Psi_{x}, \Psi_{y}\right.$ and $\left.\Psi_{z}\right)$ are identified by projective readout out pulses. Projective readout out pulses transfers the $\Psi_{x}\left(\Psi_{y}\right)$-component into a measurable population by means of a $\pi / 2$-rotation around the anti y-axis $R_{\bar{y}}(\pi / 2)$ (x-axis $R_{x}(\pi / 2)$ ). The $\Psi_{z}$ component is equivalent to the population and read out immediately. By varying time $t_{i}=t_{\text {Gate }} \cdot i / N$ in $N$ steps the full Bloch sphere trajectory is reconstructed.

It is desired to perform the projection pulses of the stroboscopy measurement within the instantaneous driving field frame of the quantum gate. In general, when realizing a SAGQG the system is off-resonantly driven and consequentially the instantaneous rotating driving field frame is varying with the detuning $\Delta(t)$ as a function of time. However, all read-out pulses are applied on-resonance in order to obtain highest accuracy. The phase difference between the two rotating frames at time $t_{i}$ of the sequence is $\varphi\left(t_{i}\right)=\Delta\left(t_{i}\right) \cdot t_{i}$ and needs to be compensated for during the waveform creation (Sec. 10.1.1).

Figure.11.5 presents the calculated and experimentally measured Bloch-sphere trajectory for a qubit initialized in the $|0\rangle$ state for a (a,b) Pauli-Z and (c,d) Pauli-X gate. In (b) and (d) the temporal evolution of the $\Psi_{x}, \Psi_{y}$ and $\Psi_{z}$ Bloch vector components are shown separately, while in (a) and (c) the information is combined to reconstruct the trajectory on the Bloch sphere itself. Numerical data are obtained by calculating the time-evolution operator $U=\mathcal{T} \exp \left(i \int_{t_{0}}^{t_{i}} H_{S}(t) \mathrm{d} t\right)$, where $\mathcal{T}$ is the time ordering operator. The experimental data are in good agreement with the simulations and remaining discrepancies are attributed to imperfect dynamic projection pulses.

Let us first take a closer look on the realization of the Pauli-Z gate (Fig. 11.5.a,b). As can be deduced from Sec. 11.3 the initial state $|0\rangle$ is equivalent to the initial energy eigenstate $\left|\lambda_{+}(0)\right\rangle=|0\rangle$ 


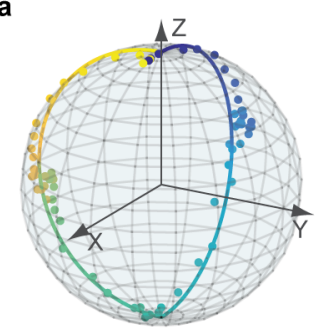

C

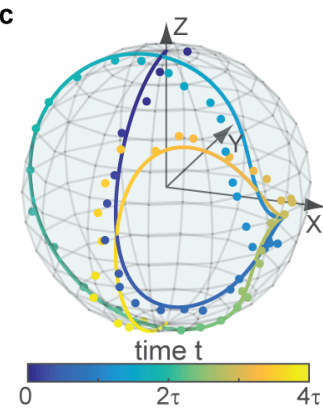

b
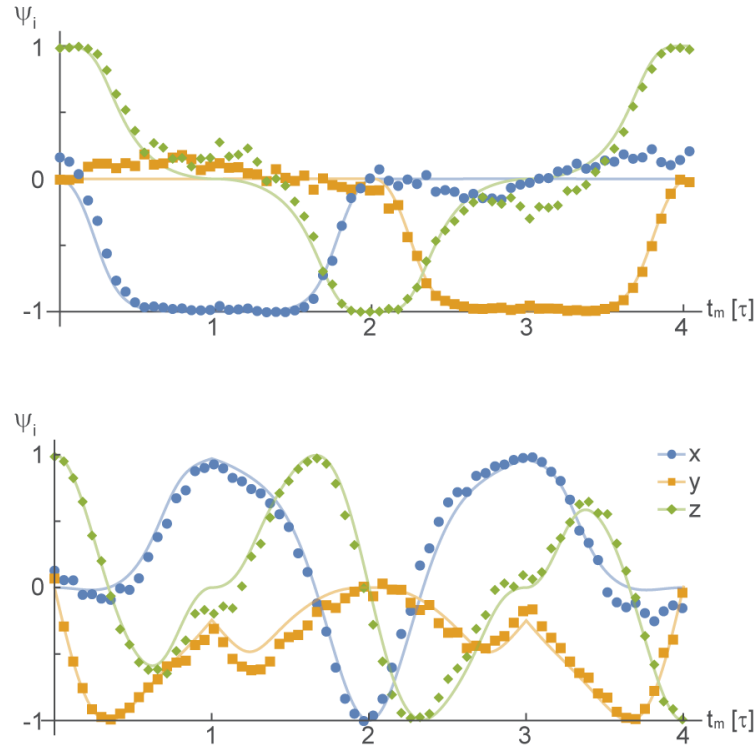

Figure 11.5.: Measured Bloch sphere trajectory. (a, c) Measured (dots) and simulated (lines) Bloch sphere trajectory of Pauli-Z and Pauli-X, respectively. The time point of each stroboscopy measurement is encoded in the colormap from blue (beginning) to yellow (end). (b, d) Bloch vector components $\psi_{x}$ (blue), $\psi_{y}$ (orange) and $\psi_{z}$ (green) of the trajectory presented in (a) and (c) versus the time in multiples of $\tau$.

of the Hamiltonian $H_{S}(0)$. The second eigenstate $\left|\lambda_{-}(0)\right\rangle=|1\rangle$ remains unoccupied. As a consequence the spin vector follows the designed "orange slice" like trajectory, as described in Sec. 11.3.2, precisely. In contrast, when realizing the Pauli-X gate the basis change (Sec. 11.3.1) causes the initial state $|0\rangle$ to be unequal to the energy eigenstates of the system $\left|\lambda_{-}(0)\right\rangle=1 / \sqrt{2}(|1\rangle+|0\rangle)$ and $\left|\lambda_{+}(0)\right\rangle=1 / \sqrt{2}(|1\rangle-|0\rangle)$. Indeed, both eigenstates are equally populated $\left|a_{+}\right|^{2}=\left|a_{-}\right|^{2}=1 / 2$. Therefore, the spin vector does not follow the path designed for the eigenstates. The complex trajectory can be interpreted as a consequence of the non-trivial parameter space utilized for the realization of the Aharonov-Anandan phase.

\subsection{Generalized SAGQG with arbitrary rotation angle}

In Sec. 11.5 it was shown that a geometric phase of $\gamma=\pi / 2$ can be acquired, realizing geometric Pauli-X and Pauli-Z gates, in a superadiabatic fashion. However, future quantum algorithms might raise the need for rotations by an arbitrary rotation angle $2 \gamma$. As stated in Sec. 11.3.2 the acquired geometric phase $\gamma$ is a function of the phase values $\varphi_{1}^{\prime}$ and $\varphi_{2}^{\prime}$ tuning the opening angle of the "orange slice" and thereby modifying the enclosed solid angle. All superadiabatically obtained phase values require the same gate duration $t_{\text {Gate }}=4 \tau$, which is in contrast to the dynamic rotation by an arbitrary angle $\alpha$ realized by a constant driving of duration $t=\alpha / \Omega_{\mathrm{R}}$. Building blocks of same duration might turn out to be a useful feature in future circuit based quantum devices, since free precession times are avoided.

By dynamically initializing the system in one of the three orthogonal states $|0\rangle, 1 / \sqrt{2}(|0\rangle-|1\rangle)$ and $1 / \sqrt{2}(|0\rangle+i|1\rangle)$ and subsequently performing the gate operation for $0 \leq \gamma \leq \pi$ the concept is proven experimentally (Fig. 11.6.a-c). The effect of the rotation about the $\mathrm{x}$-axis can be readout immediately. However, a $\pi / 2$-pulse around the $\mathrm{x}$-axis $\left(R_{x}(\pi / 2)\right)$ is applied to convert the phase 

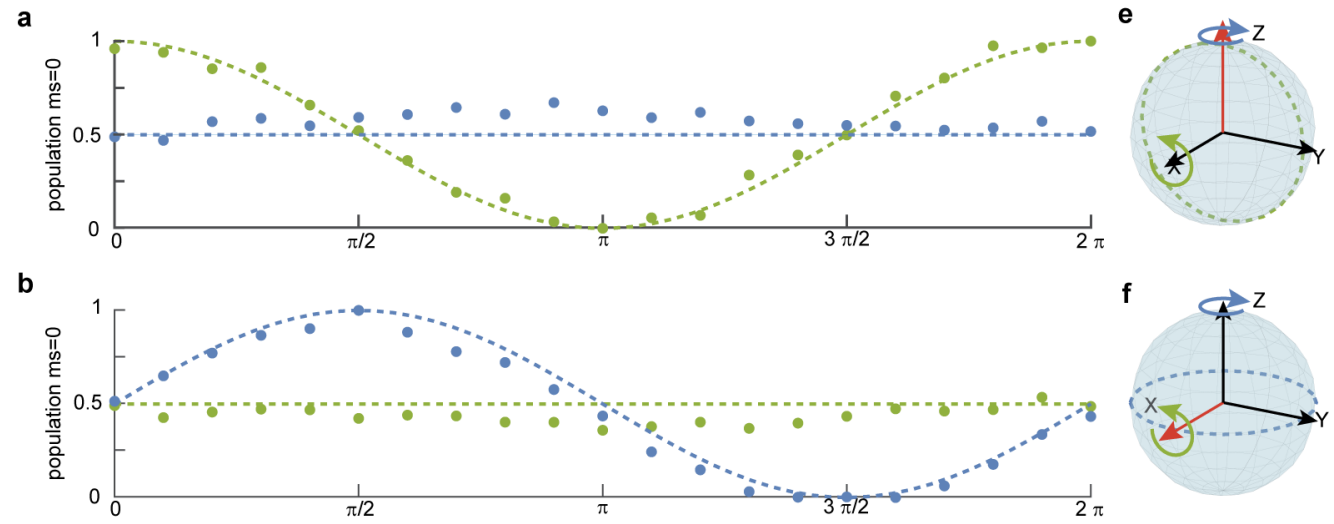

C

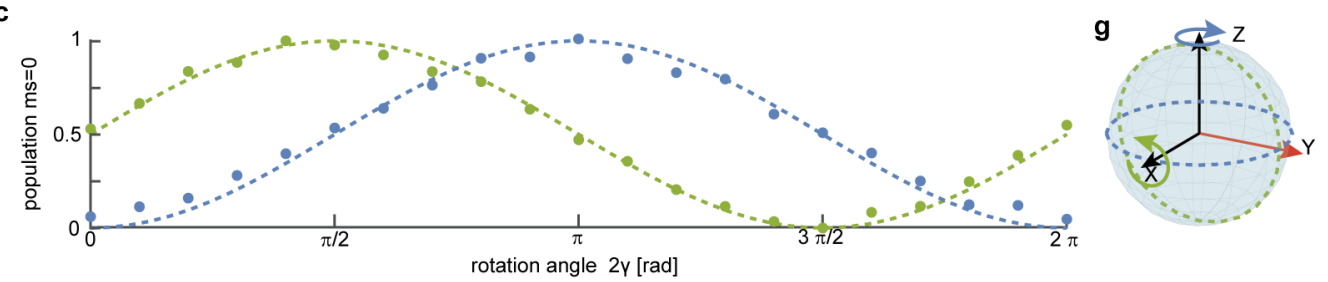

Figure 11.6.: Superadiabatic quantum gates with arbitrary phase angle. A spin initialized into $|0\rangle, 1 / \sqrt{2}(|0\rangle-|1\rangle)$ and $1 / \sqrt{2}(|0\rangle+i|1\rangle$ ) (red arrow in (e, f, g)) is rotated by an arbitrary angle around the $\mathrm{x}$ and $\mathrm{z}$-axis. (a, b, c) present the respectively measured (dots) and expected (dashed lines) population of the $|0\rangle$ state. (e, d, f) Bloch spheres indicating the initial state (red arrow) and the obtained rotation.

acquired by the rotation about the z-axis into a measurable population. The Bloch spheres in (e, f, g) illustrate the performed rotation (green, blue dashed lines) for the set of initialized states (red arrows). Obviously, a spin initialized along the rotation axis does not acquire a phase during the gate operation. (a, c, e) show the measured (dots) along with the theoretically expected (dashed lines) population of the $|0\rangle$ state depending on the geometric phase value $\gamma$. The measurement proofs that the superadiabtic geometric quantum gate modality provides a means for universal single-qubit quantum computation.

\subsection{Quantum gate fidelity}

Standard quantum process tomography (QPT) on the two-dimensional Hilbert space spanned by the computational states $|0\rangle$ and $|1\rangle$ is performed to extract the quantum gate (QG) fidelity (Sec. 9.1).

\subsubsection{Pauli gates}

Figure 11.7.a-b shows the experimentally reconstructed real part of the quantum process matrix $\chi_{\exp }$ (colored pillars), as well as the anticipated one (green frames) for the Pauli-X and Pauli-Z gate, respectively. The imaginary parts for all components are expected to be zero and the measured ones are smaller than the respective errors. Based on $\chi_{\exp }$ the QG fidelities are determined to be $F_{\mathrm{x}}^{\mathrm{SAG}}=0.973_{-0.031}^{+0.026}$ and $F_{\mathrm{z}}^{\mathrm{SAG}}=0.974_{-0.024}^{+0.021}$ for Pauli-X and Pauli-Z gate, respectively. However, due to its dynamic nature the QPT itself suffers from parameter imperfections, decoherence effects and noise. This causes an artificially reduced fidelity values. The effects of the imperfect QPT are decoupled from the gate performance by calculating the corrected fidelity $\widetilde{F}_{\mathrm{i}}=F_{\mathrm{i}} / F_{\mathrm{ID}}$. Here, $F_{\mathrm{ID}}=$ $0.979_{-0.033}^{+0.021}$ is the experimental fidelity of the identity operation, resulting in $\widetilde{F}_{\mathrm{x}}^{\mathrm{SAG}}=0.994_{-0.031}^{+0.026}$ 

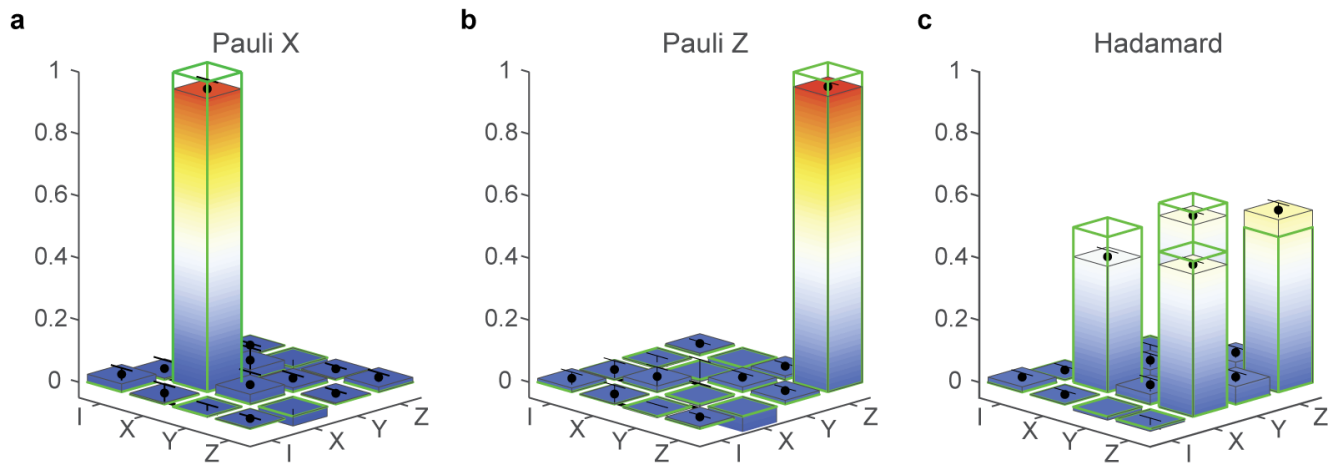

Figure 11.7.: Anticipated and reconstructed quantum process matrices. Reconstructed (colored pillars) and anticipated (green frame) real part of the quantum process matrix $\chi_{\exp }$ for (a) Pauli-X, (b) Pauli-Z and (c) Hadamard superadiabatic geometric quantum gates, respectively. Black error bars indicated the $1 \sigma$ measurement uncertainties.

and $\widetilde{F}_{\mathrm{z}}^{\mathrm{SAG}}=0.995_{-0.024}^{+0.021}$. Within our experimental uncertainties these results are compatible with an ideal fidelity, proofing the realization of a set of high fidelity QGs.

\subsubsection{Hadamard gate}

The Hadamard gate is demonstrated as an example for a short sequence of concatenated SAGQG. It corresponds to a rotation around the $(\hat{x}+\hat{z}) / \sqrt{2}$ axis by $\pi$ and is represented by the Hadamard matrix:

$$
H=\frac{1}{\sqrt{2}}\left(\begin{array}{cc}
1 & 1 \\
1 & -1
\end{array}\right) .
$$

In order to realize the Hadamard gate the non-Abelian nature of the superadiabatic geometric quantum gate is utilized. The desired operation is realized by performing a rotation of $\pi / 2$ around the y-axis $R_{y}(\pi / 2)$ and a subsequent rotation by $\pi$ around the z-axis $R_{z}(\pi)$. The combination of two superadiabatic rotations doubles the duration of the Hadamard gate compared to the one of the Pauli-X or Pauli-Z gate. Note, due to the explicit absolute time dependence in $\Delta_{\text {eff }}(t)=$ $\Delta(t)+\dot{\Delta}(t) t$ the applied detuning needs to be calculated for each gate depending on its position within the sequence individually. This represents a drawback in terms of the waveform creation time, compared to gate realizations featuring a constant or vanishing detuning. However, the circumstance of equal gate durations (see Sec. 11.6) enables the generation of a database containing quantum gates for each position of the sequence. This is particularly important for the randomized benchmarking analysis performed in Sec. 12.6.

The superadiabatic implementation results in a QG fidelity $F_{\mathrm{H}}=0.974_{-0.024}^{+0.021}$ and a corrected QG fidelity $\widetilde{F}_{\mathrm{H}}=0.992_{-0.029}^{+0.022}$ (see Fig. 11.7). As expected from Sec. 11.7.1 the combination of two superadiabatic rotations results in a high gate fidelity compatible with $F=1$. Table 11.1 summarizes the obtained QG fidelitiy values.

\section{8. $\tau$ variation}

So far, the high fidelity realization of this quantum gate concept within the accessible parameter space has been successfully demonstrated. Here, a first investigation on the robustness of the 
Table 11.1.: Measured superadiabatic geometric quantum gate fidelities. Measured quantum gate fidelities $F$ and corrected quantum gate fidelity $\widetilde{F}$ for the Pauli-X, Pauli-Z, Hadamard and identity gate. Errors correspond to the $68 \%$ confidence interval.

\begin{tabular}{ccccc} 
& Pauli-X & Pauli-Z & Hadamard & Identity \\
\hline \hline$F$ & $0.973_{-0.031}^{+0.026}$ & $0.974_{-0.029}^{+0.022}$ & $0.971_{-0.024}^{+0.021}$ & $0.979_{-0.033}^{+0.021}$ \\
$\widetilde{F}$ & $0.994_{-0.031}^{+0.026}$ & $0.995_{-0.029}^{+0.022}$ & $0.992_{-0.024}^{+0.020}$ & -
\end{tabular}

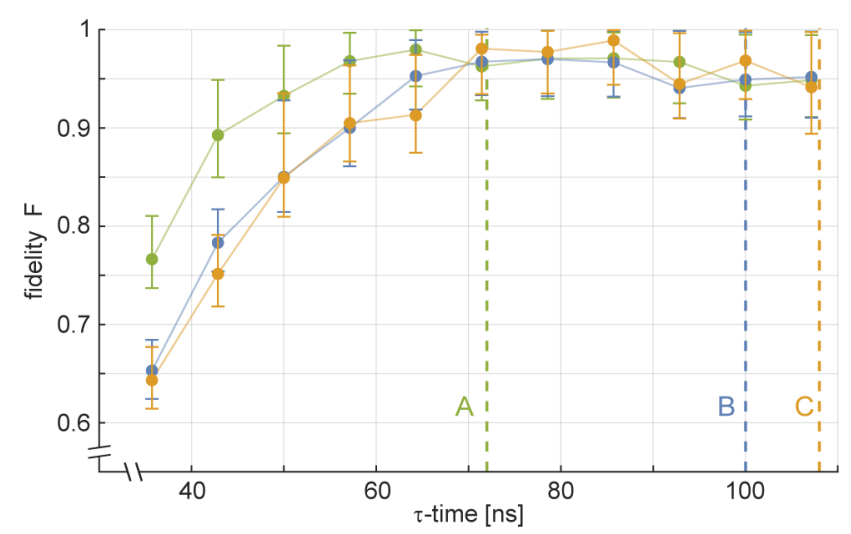

Figure 11.8.: Variation of the SAGQG timing parameter $\tau$. For three parameter sets $\left(\Delta_{0}=\right.$ $\{1.5,1.5,2\} \mathrm{MHz}$ and $\left.\Omega_{0}=\{1.5,6,8\} \mathrm{MHz}\right)$ the quantum gate fidelity is measured as a function of $\tau$. Vertical dashed lines indicate the minimal $\tau$-value which is driven efficiently $\tau_{\min }$. Solid lines represent a guide to the eye.

SAGQG is performed. The numerical analysis in Sec. 11.4 revealed the boundaries of the experimental accessible parameter space. Exceeding these boundaries results in an insufficient driving of the Hamiltonian $H_{S}(t)$.

The QG robustness is investigated by performing QPT on gate realizations with varying control parameters. Explicitly, we fix the driving field parameters $\Omega_{0}$ and $\Delta_{0}$ while varying $\tau$. Forcing $\tau<\tau_{\min }$ causes $\max _{t} \Omega_{\mathrm{S}}(t)$ to exceed the maximal Rabi frequency $\Omega_{\max }$. This condition can not be satisfied given experimental limitations on $\Omega_{\max }$ and causes an imperfect gate realization. Again, it needs to be stressed that $\tau_{\min }$ is a consequence of experimental limitations rather than theoretical constraints.

For three sets of parameters $\Delta_{0}=\{1.5,1.5,2\} \mathrm{MHz}$ and $\Omega_{0}=\{1.5,6,8\} \mathrm{MHz}$ the quantum gate fidelity is measured by standard QPT, while varying $\tau$ between $0.5 \cdot t_{\pi}$ and $1.5 \cdot t_{\pi}$ (Fig. 11.8). The in Sec. 11.4 calculated minimal $\tau$-values for the chosen parameter sets driving the Hamiltonian efficiently are $\tau_{\min }=\{72,100,107\} \mathrm{ns}$ (vertical dashed lines), respectively. Surprisingly, one observes a stable fidelity regime close to 1 for $\tau$ smaller than the expected threshold $\tau_{\min }$, even though the designed Hamiltonian is faulty driven. Only for $\tau<t_{\pi}$, representing the global $\min _{\Omega_{0}, \Delta_{0}}\left(\tau_{\min }\right)$, a significant drop of the fidelity is observed for all three parameter sets. The results represent a first indication of the intrinsic tolerance of SAGQG with respect to small control parameter imperfections. A detailed performance analysis including a comparison to other geometric and dynamic gate modalities is presented in Ch. 12 .

\subsection{Summary}

In this chapter, the concept of transitionless quantum driving was described briefly. Superadiabatic linearly chirped Gaussian pulses for population inversion have been realized. They act as an 
example for the robust acceleration of an originally adiabatic Hamiltonian. Based on the proposal by Liang et al. [81] the first experimental realization of superadiabatic geometric quantum computation was demonstrated. Qualitatively, the concept of SAGQG was realized by reconstructing the complex Bloch sphere trajectory of well defined input states and by performing rotations by arbitrary phase angles. As a quantitative measure of the gate performance, quantum process tomography was performed. Th extracted quantum gate fidelities reach the fidelity threshold needed for the efficient implementation of error-correction codes based on, e.g., surface codes [164, 165]. Additionally, numeric simulations considering realistic experimental boundary conditions show that the SAGQGs can be realized within a minimal gate duration of $t_{\text {Gate }}=4 \cdot t_{\pi}$, outperforming each adiabatic approach significantly. Finally, the variation of experimental control parameter indicate a stable performance over a large range of timing parameter and an intrinsic robustness with respect to control parameter imperfections. 


\section{Benchmarking quantum gates}

The realization of different quantum gate modalities naturally raises the question, which of the concepts results in the most robust quantum gate $(\mathrm{QG})$ implementation for QECCs in NISQs. However, a comprehensive, comparative performance analysis of geometric QGs as well as dynamic QGs has not been conducted. Here, the robustness of a variety of QG modalities, including dynamic, Abelian geometric, non-adiabatic holonomic and superadiabatic geometric quantum gates, are analysed with respect to constant driving field parameter detunings. Additionally, a randomized benchmarking $(\mathrm{RB})$ analysis investigates the error scaling for the different modalities for the $\mathrm{NV}$ center in diamond.

\subsection{Considered quantum gates modalities}

In order to cover a broad range of modalities for the realization of high-fidelity QGs the comparative, quantitative and qualitative benchmark analysis is performed between dynamic quantum gate (DYN), Abelian geometric quantum gate (AGQG), non-adiabatic non-Abelian holonomic quantum gate (HQG) and superadiabatic geometric quantum gate (SAGQG). While the SAGQG is discussed in detail in Ch. 11, we briefly describe the experimental implementation of DYN, AGQG and HQG in the following.

\subsubsection{Dynamic quantum gates}

The realized set of dynamic single-qubit gates is defined on the two-dimensional Hilbert space spanned by $|0\rangle$ and $|-\rangle$. Rotations around the $\mathrm{x}$ and $\mathrm{y}$-axis are performed by resonant driving fields of appropriate amplitude $\Omega$, phase $\varphi$ and duration $t_{\text {Gate }}$. Particularly, $\pi$ and $\pi / 2$-pulses are realized by pulses of length $t_{\pi}=\pi / \Omega$ and $t_{\frac{\pi}{2}}=\pi /(2 \Omega)$. In contrast, the dynamic phase-shift gate is implemented in a Ramsey experiment like fashion $\left(R_{x}(\pi / 2)-\tau_{\text {free }}^{\delta}-R_{x}^{\delta}(\pi / 2)\right)$, where $\delta$ represents a detuning of the resonance and $\tau_{\text {free }}^{\delta}$ a time of free precession. The duration of the sequence is limited by the detuning $\delta$, which acquires the wanted phase $\varphi$ during the time $\tau_{\text {free }}^{\delta}=\varphi / \delta$. Note, the detuning $\delta$ causes an increased generalized Rabi frequency $\Omega^{\prime}=\sqrt{\Omega^{2}+\delta^{2}}$ and a tilt of the rotation axis. Thereby the faulty second $\pi / 2$-pulse results in an erroneous gate operation. As a consequence, the choice of $\delta$ represents a trade off between fast gate operation and designed fidelity. The utilized detuning of $\delta=1 \mathrm{MHz}$ results in a free precession time of $\tau_{\text {free }}^{\delta}=500 \mathrm{~ns}$ and a designed fidelity of $F=0.990$ for the Pauli-Z gate.

\subsubsection{Abelian geometric quantum gate}

One of the simplest geometric phase based quantum gates is the phase shift gate realized by the subsequent application of two $\pi$-pulses. The accumulated phase $\gamma$ is defined by the phase difference $\Delta \varphi=\gamma / 2$ of the $\pi$-pulses, while the absolute values of the driving field phases are not of importance, and are set to $\varphi_{1}=0$ and $\varphi_{2}=\Delta \varphi$. The resulting trajectory resembles the "orange slice"-like trajectory discussed in Sec. 11.3.2. Note, the complete gate sequence represents a closed 
Table 12.1.: HQG driving field parameter. Two subsequently applied HQG $n$ and $m$ realize arbitrary single qubit quantum operations. Here, the parametrization angles $\phi^{n, m}, \theta^{n, m}$ and Rabi weights $\gamma_{ \pm}^{n, m}$ of all in the scope of the thesis utilized quantum gates are given.

\begin{tabular}{|c|c|c|c|c|c|c|c|c|}
\hline Gate & $\phi^{n}$ & $\theta^{n}$ & $\gamma_{-}^{n}$ & $\gamma_{+}^{n}$ & $\phi^{m}$ & $\theta^{m}$ & $\gamma_{-}^{m}$ & $\gamma_{+}^{m}$ \\
\hline Identity & 0 & $-\frac{\pi}{2}$ & $-1 / \sqrt{2}$ & $-1 / \sqrt{2}$ & 0 & $-\frac{\pi}{2}$ & $-1 / \sqrt{2}$ & $-1 / \sqrt{2}$ \\
\hline Identity & 0 & $-\frac{\pi}{2}$ & $-1 / \sqrt{2}$ & $-1 / \sqrt{2}$ & $2 \pi$ & $-\frac{\pi}{2}$ & $-1 / \sqrt{2}$ & $-1 / \sqrt{2}$ \\
\hline$R_{x}(\pi)$ & $\pi$ & $-\frac{\pi}{2}$ & $-1 / \sqrt{2}$ & $-1 / \sqrt{2}$ & - & - & - & - \\
\hline$R_{y}(\pi)$ & $-\frac{\pi}{2}$ & $-\frac{\pi}{2}$ & $-e^{-i \frac{\pi}{2}} / \sqrt{2}$ & $-1 / \sqrt{2}$ & - & - & - & - \\
\hline$R_{z}(\pi)$ & 0 & 0 & 0 & -1 & - & - & - & - \\
\hline$R_{\bar{x}}(\pi)$ & $-\pi$ & $\frac{\pi}{2}$ & $e^{-i \pi} / \sqrt{2}$ & $-1 / \sqrt{2}$ & - & - & - & - \\
\hline$R_{\bar{y}}(\pi)$ & $\frac{\pi}{2}$ & $-\frac{\pi}{2}$ & $-e^{i \frac{\pi}{2}} / \sqrt{2}$ & $-1 / \sqrt{2}$ & - & - & - & - \\
\hline$R_{\bar{z}}(\pi)$ & 0 & $\pi$ & 1 & 0 & - & - & - & - \\
\hline$R_{x}(\pi / 2)$ & $-\frac{\pi}{2}$ & $-\frac{\pi}{4}$ & $-e^{-i \frac{\pi}{2}} \sin \left(\frac{\pi}{8}\right)$ & $-\cos \left(\frac{\pi}{8}\right)$ & 0 & $\pi$ & 1 & 0 \\
\hline$R_{y}(\pi / 2)$ & 0 & $\frac{\pi}{4}$ & $\sin \left(\frac{\pi}{8}\right)$ & $-\cos \left(\frac{\pi}{8}\right)$ & 0 & $\pi$ & 1 & 0 \\
\hline$R_{z}(\pi / 2)$ & 0 & $\frac{\pi}{2}$ & $1 / \sqrt{2}$ & $-1 / \sqrt{2}$ & $-\frac{\pi}{4}$ & $\frac{\pi}{2}$ & $e^{-i \frac{\pi}{4}} / \sqrt{2}$ & $-1 / \sqrt{2}$ \\
\hline$R_{\bar{x}}(\pi / 2)$ & $\frac{\pi}{2}$ & $\frac{3 \pi}{4}$ & $e^{i \frac{\pi}{2}} \cos \left(\frac{\pi}{8}\right)$ & $-\sin \left(\frac{\pi}{8}\right)$ & 0 & $\pi$ & 1 & 0 \\
\hline$R_{\bar{y}}(\pi / 2)$ & 0 & $\frac{3 \pi}{4}$ & $\cos \left(\frac{\pi}{8}\right)$ & $-\sin \left(\frac{\pi}{8}\right)$ & 0 & $\pi$ & 1 & 0 \\
\hline$R_{\bar{z}}(\pi / 2)$ & 0 & $\frac{\pi}{2}$ & $1 / \sqrt{2}$ & $-1 \sqrt{2}$ & $\frac{\pi}{4}$ & $-\frac{\pi}{2}$ & $-e^{i \frac{\pi}{4}} / \sqrt{2}$ & $-1 / \sqrt{2}$ \\
\hline
\end{tabular}

loop in projective Hilbert space acquiring an Abelian Aharonov-Anandan phase [118]. Dynamic contributions are suppressed by employing the two-loop method (Sec. 8.4). The conceptually simple and fast $\left(t_{\text {Gate }}=2 \cdot t_{\pi}\right)$ AGQG provides a reference to the more complex SAGQG phase shift gate. IN this way it can be probed if the increased QG complexity results in an improved robustness and higher quantum gate fidelity.

\subsubsection{Non-Abelian non-adiabatic quantum gates}

Due to its holonomic nature, the non-Abelian non-adiabatic holonomic quantum gate (HQG) is performed on the at least three-dimensional Hilbert space (Sec. 8.5). Here, the original approach by E. Sjöqvist [127] defined on the Hilbert space spanned by $|0\rangle,|-\rangle$ and $|+\rangle$ is followed (Sec. 8.6). For simplicity, the two driving fields feature a constant pulse envelope $\Omega(t)=\Omega_{0}$. Processes acting on the three-dimensional Hilbert space possess a quantum process matrix $\chi$ of dimensions $9 \times 9$ and standard quantum tomography requires 9 preparation and 9 projection pulses [141].

The driving field parameters are derived utilizing Eq. 8.7 and Eq. 8.8. Standard Pauli gates are implemented by a single driving pulse. Rotations by an angle of $\pi / 2$ are realized by the subsequent application of two $\pi$-rotations $n$ and $m$ around suitably chosen axis. For example, a $\pi / 2$-rotation around $\hat{x}(\hat{y})$ is achieved by a $\pi$-rotation around $[\hat{y}+\hat{z}] / \sqrt{2}([\hat{x}+\hat{z}] / \sqrt{2})$ followed by a $\pi$-rotation around $\hat{z}$, respectively. Equivalently to the AGQG realization, the phase-shift gate obtaining a geometric phase $\gamma$ is obtained by two $\pi$-pulses with a rotation axis in the xy-plain and a phase difference of $\Delta \varphi=\gamma / 2$. The parameter for rotations around the anti-axis follow analogously. In Tab. 12.1 the driving field parameters for all $\pi$ and $\pi / 2$-rotation utilized in the scope of this thesis are given explicitly. 
Table 12.2.: Gate parameters of the benchmarking analysis. The parameters were chosen to minimize the gate duration $t_{\text {Gate }}$, while not exceeding the maximal output power $P_{\text {out }}^{\max }=$ 0.92 .

\begin{tabular}{lccccc} 
& $\Omega_{-}^{\max }[\mathrm{MHz}]$ & $\Omega_{+}^{\max }[\mathrm{MHz}]$ & $P_{\text {out }}^{\max }[\mathrm{norm}]$. & $\bar{P}_{\text {out }}[$ norm. $]$ & $t_{\text {Gate }}[\mathrm{ns}]$ \\
\hline \hline DYN Pauli-X & 7 & - & 0.92 & 1 & 71 \\
DYN Pauli-Z & 7 & - & 0.92 & 1 & 571 \\
AGQG Pauli-Z & 7 & - & 0.92 & 0.12 & 142 \\
SAGQG Pauli-X & 7 & - & 0.92 & 0.77 & 320 \\
SAGQG Pauli-Z & 7 & - & 0.92 & 0.77 & 320 \\
HQG Pauli-X & 2.76 & 2.76 & 0.92 & 1 & 256 \\
HQG Pauli-Z & 3.9 & - & 0.79 & 0.86 & 256
\end{tabular}

\subsection{Boundary conditions}

The design of an experiment representing a fair comparison of the robustness of different quantum gate modalities is challenging, due to the varying number of involved levels and driving fields, as well as the extensive accessible parameter space. By exploiting the whole parameter space it is likely to find configurations where one or the other QG modality reveals superior robustness with respect to the assumed main source of error. In the following, the experimentalist point of view is investigated, which is typically aiming for fastest gate execution limited by the maximal available driving field strength $\Omega_{\max }$. The boundary conditions might vary depending on the chosen experimental platform. Hence, the results presented here do not represent a genuine truth, but need to be adjusted for the considered physical system.

Here, the experimental apparatus (Ch. 5 and Ch. 10) is limited by the output power of the arbitrary waveform generator (AWG) (more precisely the microwave amplifier). The microwave source provides the driving fields for all transitions at the same time through a single channel. Inhomogeneities in the system specific microwave response cause the driving field strength to vary for the transitions $|0\rangle \leftrightarrow|-\rangle\left(\Omega_{\max }^{-} \approx 7.57 \mathrm{MHz}\right.$ at $\left.\omega=1.73 \mathrm{GHz}\right)$ and $|0\rangle \leftrightarrow|+\rangle\left(\Omega_{\max }^{+} \approx 4.92 \mathrm{MHz}\right.$ at $\omega=4.01 \mathrm{GHz}$ ). Measuring in a stable experimental regime is guaranteed by setting $\Omega_{\max }=$ $7 \mathrm{MHz}$ for the $|0\rangle \leftrightarrow|-\rangle$ transition corresponding to $92 \%$ of the maximal output power $P_{\text {out }}^{\max }$. For the realization of the HQG the Rabi frequency is set to $\Omega_{0}=3.9 \mathrm{MHz}$ resulting in $P_{\text {out }}^{\max }=0.92$ for standard Pauli gates, as well.

In the following all gate parameter have been chosen to minimize $t_{\text {Gate }}$ for the given $P_{\text {out }}^{\max }$. Table 12.2 summarizes the maximal driving field parameter on both transition $\left(\Omega_{-}^{\max }, \Omega_{+}^{\max }\right)$. Additionally, the maximal output power $P_{\text {out }}^{\max }$, the average output power $\bar{P}_{\text {out }}$ normalized to $P_{\text {out }}^{\max }=0.92$ (a measure for the introduced heating) and the gate duration $t_{\text {Gate }}$ for all realized gate modalities are given. All geometric phase based non-adiabatic approaches are realized on time scales comparable to the once of the dynamic gate set. The SAGQG approach potentially benefits from a lower average output power $\bar{P}_{\text {out }}$, which reduces the heating of the system.

\subsection{Gate fidelity comparison}

Standard quantum process tomography (QPT) measurements (Sec. 9.1) extracting the quantum gate fidelity of the DYN, AGQG, SAGQG and HQG are performed utilizing the same nitrogen vacancy center under ambient conditions. The choice of identical experimental conditions allows for a quantitative performance comparison. Table 12.3 summarises the achieved corrected gate 
Table 12.3.: Measured quantum gate fidelities. Experimentally obtained corrected quantum gate fidelities $\widetilde{F}$ for dynamic (DYN), Abelian geometric (AGQG), superadiabatic geometric (SAGQG) and non-adiabatic holonomic geometric (HQG) quantum gates.

\begin{tabular}{ccccc} 
& DYN & AGQG & SAGQG & HQG \\
\hline \hline$\widetilde{F}_{\mathrm{X}}$ & $0.998_{-0.030}^{+0.023}$ & - & $0.994_{-0.031}^{+0.026}$ & $1.000_{-0.121}^{+0.000}$ \\
$\widetilde{F}_{\mathrm{Z}}$ & $0.997_{-0.045}^{+0.023}$ & $1.000_{-0.040}^{+0.033}$ & $0.995_{-0.024}^{+0.021}$ & $1.018_{-0.120}^{+0.000}$
\end{tabular}

fidelities for all four quantum gate modalities. Within the experimental uncertainties all corrected gate fidelities are compatible with $\widetilde{F}=1$. Induced by the more complex QPT, the error of the HQG is approximately three times larger than the one of the gates realized on the two-dimensional Hilbert space. The achieved QG fidelities reach a quality where differentiation is prohibited by experimental uncertainties of the QPT. However, literature suggest geometric phase based QGs provide a performance advantage for imperfectly driven and noise quantum register (Sec. 8.7). In the following, the quantum gate fidelity depending on a faulty driving field Hamiltonian and for concatenated gate sequences, where the influence of noise can not be ignored, is investigated.

\subsection{Numerical robustness analysis}

In general, one distinguishes between low frequency and high frequency noises acting on the investigated qubit. Due to the non-adiabaticity of all considered concepts the interaction with the surrounding spin bath, which in diamond is known to decay with increasing frequency [42, 166], is negligible for a single gate realization. Only noise at a frequency $f$ in the order of the inverse gate duration $1 / t_{\text {Gate }}$ is expected to disturb the system significantly. However, the determination of the exact noise spectra is a challenging task and a current field of research (e.g., $[167,168,169])$. Here, the low frequency noise limiting case (DC noise) is considered, while in Sec. 12.6 the effects of higher frequency noise are investigated indirectly by measuring the error scaling of concatenated gate sequences.

In a theoretical approach, the quantum gate fidelity is investigated numerically for imperfect driving Hamiltonians $H(\eta, \epsilon)$. Here, $\eta$ is the detuning of the driving field frequency $\omega_{D}$ from the resonance $\omega_{0}$ and $\epsilon$ describes a faulty scaling of the Rabi frequency $\Omega_{0}$. Systematic detunings $\eta$ and $\epsilon$ correspond to experimental imprecisions in the determination of the resonance $\omega_{0}$ and Rabi frequency $\Omega$ or the influence of a slowly drifting system. Errors of this kind are inherent in every quantum system. However, the distinctiveness and dominant contribution will vary with the utilized physical platform.

\subsubsection{Implementation}

An estimate on the quantum gate fidelity is obtained by numerically computing the full system evolution for the Hamiltonian $H(\eta, \epsilon)$ and calculating the expected outcome of each preparation/projection combination. Subsequently, the results of this numerically performed standard QPT are analysed by the evaluation routine described in Sec. 9.1.

The system evolution is described by the time evolution operator

$$
U_{\eta, \epsilon}^{\text {sim }}\left(t_{\text {Gate }}\right)=\mathcal{T} \exp \left(\frac{i}{\hbar} \int_{0}^{t_{\text {Gate }}} H(t, \eta, \epsilon) \mathrm{d} t\right),
$$


where $\mathcal{T}$ is the time ordering operator and $t_{\text {Gate }}$ the gate duration. While it is trivial to solve the integral for time independently driven quantum gates, in the case of a time-dependent driving it needs to be evaluated numerically. By calculating the Riemann sum in $n$ steps the time evolution operator follows as

$$
U_{\eta, \epsilon}^{\mathrm{sim}}(T)=\prod_{n} \mathcal{T} \exp \left(\frac{i}{\hbar} H\left(t_{n}, \eta, \epsilon\right) \cdot t_{\mathrm{s}}\right)
$$

where $t_{\mathrm{s}}=t_{\text {Gate }} / n$. In order to account for the granularity of the AWG $n$ was set to $n=t_{\text {Gate }} / s$ with $s$ the inverse AWG sampling rate. Based on the time evolution operator of the detuned Hamiltonian the final state $\left|\Psi_{f}^{j, k}\right\rangle$ for each QPT preparation, projection combination is calculated according to:

$$
\left|\Psi_{f}^{j, k}\right\rangle=U_{k}^{\mathrm{proj}} \cdot U_{\eta, \epsilon}^{\mathrm{sim}}(T) \cdot U_{j}^{\mathrm{prep}}\left|\Psi_{i}\right\rangle
$$

Here, $\left|\Psi_{i}\right\rangle$ is the initialized state $|0\rangle$ and $U_{j}^{\text {prep }}\left(U_{k}^{\text {proj }}\right)$ is the ideal time evolution operator of the QPT preparation (projection) pulse, respectively. Note, $j, k \in\{1 \ldots 4\}$ for gates performed on the two-dimensional Hilbert space (DYN, AGQG, SAGQG) and $j, k \in\{1 \ldots 9\}$ for the gates realized in the three-dimensional Hilbert space (HQG).

The simulated QPT outcome is subsequently evaluated by the routine presented in Sec. 9.1 and reveals the expected quantum gate fidelity $F$. Since $U_{\eta, \epsilon}^{\operatorname{sim}}(T)$ is a unitary matrix for all $\epsilon$ and $\eta$ the evaluation of the numerical measurement outcome Eq. 12.3 will always result in a physical process matrix $\chi$ and no maximum likelihood estimation, as for the experimental data set, is required. As a consequence, the required computational resources to investigate a large parameter space of $\eta$ and $\epsilon$ remain in an adequate regime.

\subsubsection{Detuning definition}

In the following, the Hamiltonian for all QGs with detuning $\eta$ and $\epsilon$ are given explicitly. The orthonormal basis is spanned by $|0\rangle=(1,0,0)^{T},|-\rangle=(0,1,0)^{T}$ and $|+\rangle=(0,0,1)^{T}$, where $T$ is the conjugate transpose. The most general expression of the Hamiltonian in the driving field frame is:

$$
H\left(t, \Omega_{ \pm}, \Delta_{ \pm}\right)=\frac{\hbar}{2}\left(\begin{array}{ccc}
0 & \Omega_{-}(t) \cdot e^{i \varphi_{-}(t)} & \Omega_{+}(t) \cdot e^{i \varphi_{+}(t)} \\
\Omega_{-}(t) \cdot e^{-i \varphi_{-}(t)} & -2 \cdot \Delta_{-}(t) & 0 \\
\Omega_{+}(t) \cdot e^{-i \varphi_{+}(t)} & 0 & -2 \cdot \Delta_{+}(t)
\end{array}\right) .
$$

Obviously, for DYN, AGQG and SAGQG utilizing the two-dimensional Hilbert space the driving field parameter of the $|0\rangle \leftrightarrow|+\rangle$ transition are set to $\Delta_{+}=\Omega_{+}=0$ resulting in the effective two-level Hamiltonian:

$$
H(t, \Omega, \Delta)=\frac{\hbar}{2}\left(\begin{array}{cc}
0 & \Omega(t) \cdot e^{i \varphi(t)} \\
\Omega(t) \cdot e^{-i \varphi(t)} & -2 \cdot \Delta(t)
\end{array}\right) .
$$

The detuning from the resonance $\eta$ is the difference of the driving field and resonance frequency $\eta=\omega_{\mathrm{D}}-\omega_{0}$. An artificial detuning $\eta$ is introduced by replacing $\Delta_{-}(t)$ and $\Delta_{+}(t)$ by the respective 

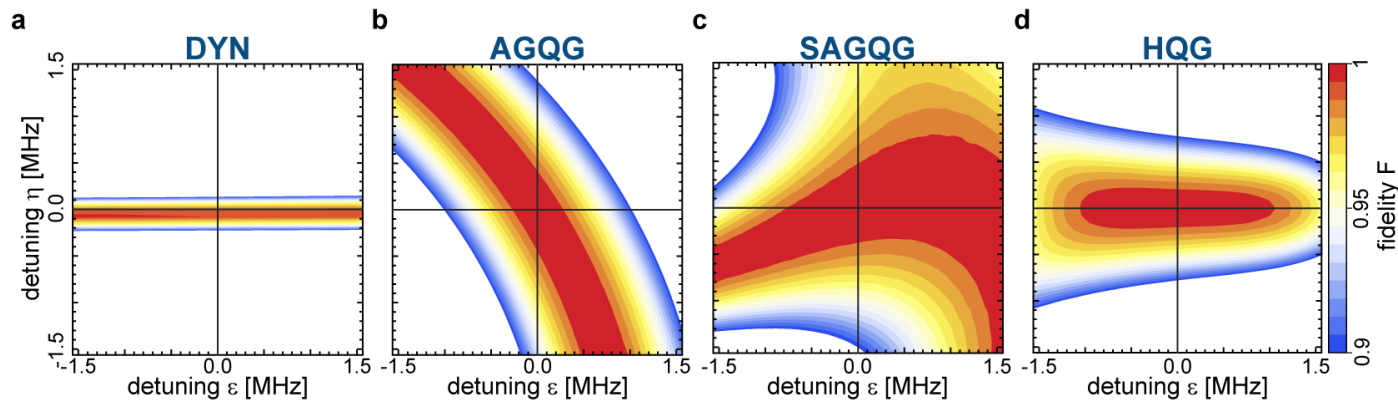

Figure 12.1.: Numeric robustness calculation for Pauli-Z. Expected quantum gate fidelity $F$ for gate operations driven by an imperfect Hamiltonian $H(\eta, \epsilon)$, where $\eta$ represents a detuning from the resonance $\omega_{0}$ and $\epsilon$ is the detuning of the Rabi frequency $\Omega_{0}$. Numerical calculations are performed for the Pauli-Z gate realized by (a) DYN, (b) AGQG, (c) SAGQG and (d) HQG, respectively.

expressions:

$$
\begin{aligned}
\Delta_{\mathrm{DYN}}(t, \eta) & =\eta \\
\Delta_{\mathrm{AGQG}}(t, \eta) & =\eta \\
\Delta_{\mathrm{SAGQG}}(t, \eta) & =\Delta(t)+\eta \\
\Delta_{\mathrm{HQG},-}(t, \eta) & =\eta_{-}, \quad \Delta_{\mathrm{HQG},+}(t, \eta)=\eta_{+}
\end{aligned}
$$

In contrast, Rabi measurements relate the maximal driving field amplitude to the corresponding Rabi frequency $\Omega_{\max }$, utilizing the linear relation to scale the microwave amplitude $\Omega_{0}$ in the subsequent experiments. A by $\epsilon$ incorrectly determined $\Omega_{\max }$ leads to an erroneous scaling of $\Omega_{0}$ and is incorporated in the Hamiltonian by setting

$$
\begin{aligned}
\Omega_{\mathrm{DYN}}(t, \epsilon) & =\Omega_{0}(t) \cdot\left(1+\epsilon / \Omega_{\max }\right), \\
\Omega_{\mathrm{AGQG}}(t, \epsilon) & =\Omega_{0}(t) \cdot\left(1+\epsilon / \Omega_{\max }\right), \\
\Omega_{\mathrm{SAGQG}}(t, \epsilon) & =\Omega_{\mathrm{S}}(t) \cdot\left(1+\epsilon / \Omega_{\max }\right), \\
\Omega_{\mathrm{HQG}}^{-}(t, \epsilon) & =\Omega_{0}(t) \gamma_{-} \cdot\left(1+\epsilon_{-} / \Omega_{\max }^{-}\right), \quad \Omega_{\mathrm{HQG}}^{+}(t, \epsilon)=\Omega_{0}(t) \gamma_{+} \cdot\left(1+\epsilon_{+} / \Omega_{\max }^{+}\right),
\end{aligned}
$$

respectively. In the presence of two in general independently detuned driving fields a continuum of detuning combinations is possible. For simplicity, only the cases of same absolute detuning value $\left|\eta_{-}\right|=\left|\eta_{+}\right|\left(\left|\epsilon_{-}\right|=\left|\epsilon_{+}\right|\right)$with same sign $\eta_{\mathrm{ss}}\left(\epsilon_{\mathrm{ss}}\right)$ and opposite sign $\eta_{\mathrm{os}}\left(\epsilon_{\mathrm{os}}\right)$ are considered. Additionally, the different maximal applicable Rabi frequencies for the transitions $|0\rangle \leftrightarrow|-\rangle\left(\Omega_{\max }^{-}\right)$ and $|0\rangle \leftrightarrow|+\rangle\left(\Omega_{\max }^{+}\right)$, which are utilized as a scaling factor, need to be considered. For convenience, we set $\Omega_{\max }^{-}=\Omega_{\max }^{+}=6 \mathrm{MHz}$ assuming an homogeneous frequency response of the system.

\subsubsection{Results}

In Fig. 12.1 and Fig. 12.2 the calculated fidelity $F$ for the selected QG modalities depending on the detuning parameter $\eta$ and $\epsilon$ for the Pauli-X and Pauli-Z gate is presented. For an easy comparison, the colormap within all plots is normalized to the fidelity interval $F \in[0.9,1]$. The detunings $\epsilon$ and $\eta$ are in the range of $\pm 1.6 \mathrm{MHz}$. Abelian geometric quantum gates are limited to commuting operations around the $\mathrm{z}$-axis. The holonomic Pauli-X gate requires two driving fields and the four cases $\eta_{\mathrm{ss}} \epsilon_{\mathrm{ss}}, \eta_{\mathrm{os}} \epsilon_{\mathrm{ss}}, \eta_{\mathrm{ss}} \epsilon_{\mathrm{os}}$ and $\eta_{\mathrm{os}} \epsilon_{\mathrm{os}}$ (Sec. 12.4.2) are distinguished (c-f). 

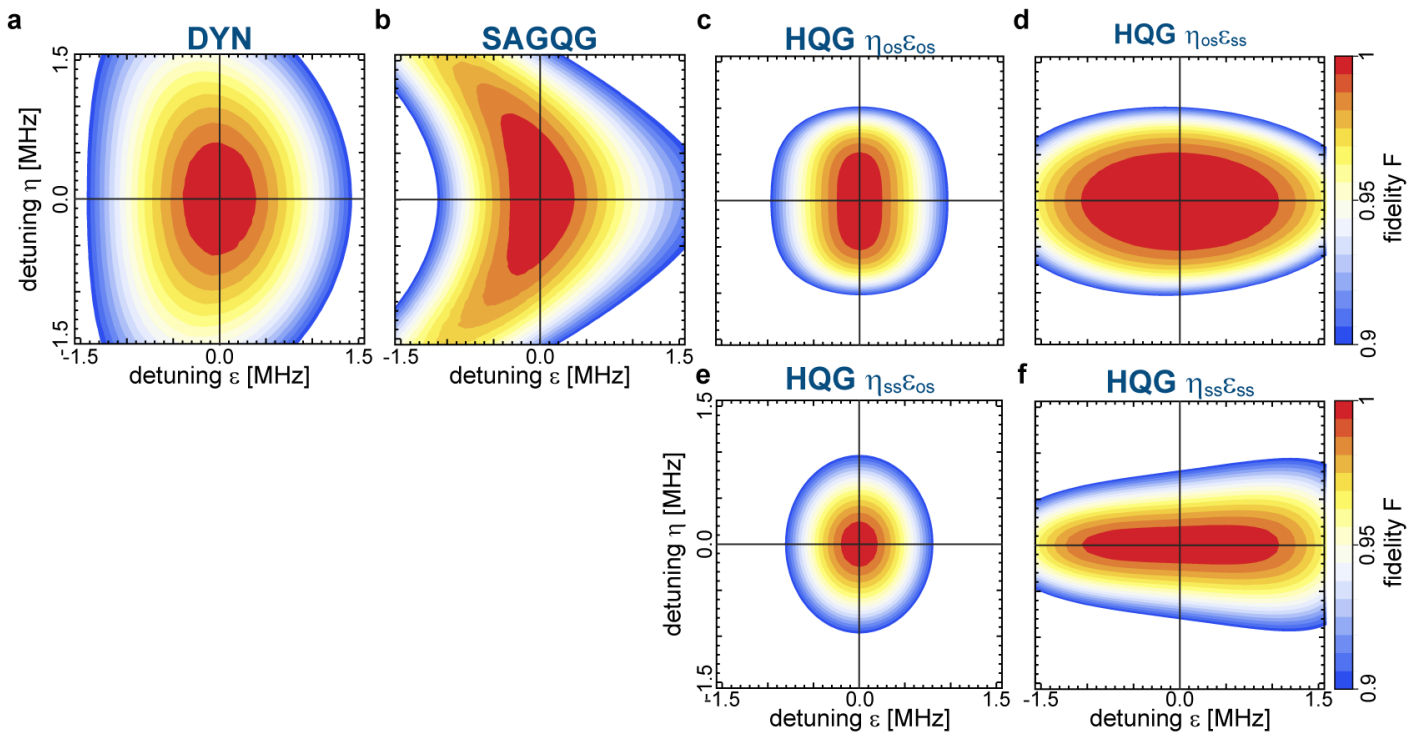

Figure 12.2.: Numeric robustness calculation for Pauli-X. Expected quantum gate fidelity $F$ for gate operations driven by an imperfect Hamiltonian $H(\eta, \epsilon)$, where $\eta$ represents a detuning from the resonance $\omega_{0}$ and $\epsilon$ is the detuning of the Rabi frequency $\Omega_{0}$. Numerical calculations are performed for the Pauli-X gate realized by (a) DYN, (b) SAGQG and (c-f) HQG, respectively.

The robustness strongly varies not only with the chosen QG modality but also with the realized QG (here Pauli-X, Pauli-Z). Since the lines of equal fidelity do not follow any mutual or regular shape, a detailed comparison between the gate realizations is difficult but some general conclusions can be drawn.

Most conspicuous is the poor performance of the DYN Pauli-Z gate (Fig. 12.1.a), which is highly sensitive to the detuning $\eta$. Due to the intrinsic detuning this modality never yields ideal fidelity. The AGQG, SAGQG and HQG counterparts appear to be intrinsically more robust (b-d). Surprisingly, the dynamic Pauli-X gate realization (Fig. 12.2.a) is compared to the other modalities similarly robust and superior robustness depends on the dominant detuning parameter. The HQG robustness is mainly determined by the assumed sign of the detuning parameters (same sign or opposite sign). While the same sign detuning $\left(\eta_{\mathrm{ss}} \epsilon_{\mathrm{ss}}\right)$ results in a high tolerance with respect to the detuning parameter $\epsilon$, its sensitivity to the detuning of $\eta$ is comparatively high (f). The robustness of the purely opposite sign case $\left(\eta_{\mathrm{os}} \epsilon_{\mathrm{os}}\right)$ behaves vica versa (c). The mixed detuning realizations $\eta_{\mathrm{os}} \epsilon_{\mathrm{ss}}$ and $\eta_{\mathrm{ss}} \epsilon_{\mathrm{os}}$ can be interpreted as projection of the $\eta_{\mathrm{ss}} \epsilon_{\mathrm{ss}}, \eta_{\mathrm{os}} \epsilon_{\mathrm{os}}$ cases, resulting in the most reliable performance for $\eta_{\mathrm{os}} \epsilon_{\mathrm{ss}}(\mathrm{d})$. Worst robustness is expected for the $\eta_{\mathrm{ss}} \epsilon_{\mathrm{os}}$ case (e).

One might distinguish between symmetric (HQG, DYN Pauli-X) and asymmetric (AGQG, DYN Pauli-Z, SAGQG) fidelity response with respect to the detuning parameter $\eta$. The symmetric cases can be represent by a Rabi oscillation. Hence, a detuning $\eta$ from the resonance causes a tilt of the rotation axis and a change of the generalized Rabi frequency $\Omega=\sqrt{\Omega_{0}^{2}+\eta^{2}}$. The generalized Rabi frequency $\Omega$ does not depend on the sign of the detuning $\eta$ and the tilt angle is of equivalent absolute value. Gates detuned by $+\eta$ and $-\eta$ and performed on the same input state do not result in the same output state $\left|\Psi^{\text {out }}\right\rangle$. But both $\left|\Psi^{\text {out }}\right\rangle$ feature the same distance to the anticipated final state and hence the same overall gate fidelities are equivalent. Since for $\eta=0$ the difference between anticipated and covered rotation angle is of same absolute value, the fidelity response is symmetric with respect to $\epsilon$. Only for $\eta \neq 0$ the symmetry is broken and different fidelity values are observed for $\epsilon$, as a consequence of the combined differences in tilt and rotation angle. 


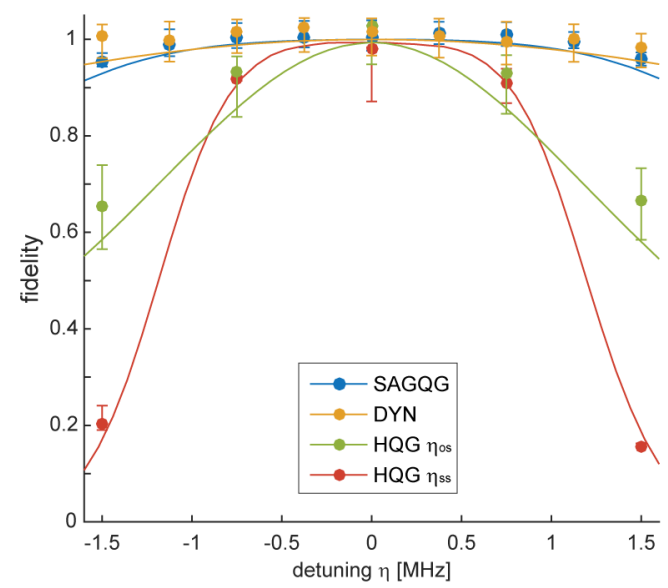

b

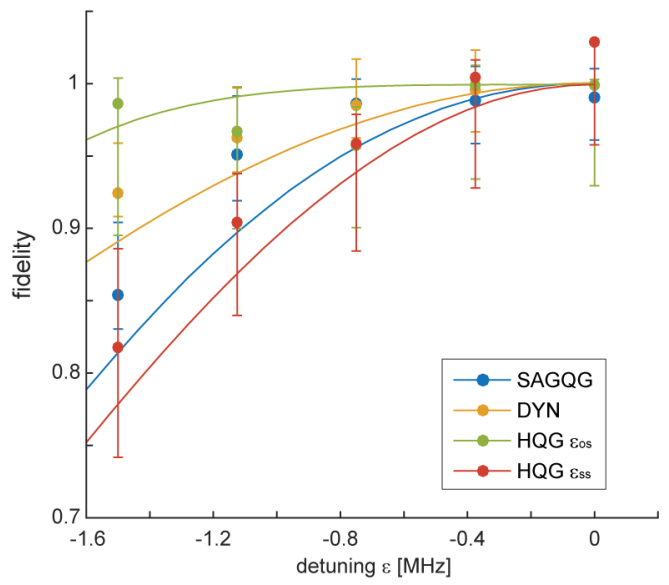

Figure 12.3.: Pauli-X gate fidelity as a function of the detuning $\eta$ and $\epsilon$. Calculated (solid lines) and measured (dots with error-bars) quantum gate fidelity of the Pauli-X operation for dynamic (orange), superadiabatic (blue) and holonomic (green, red) gate modality depending on the detuning parameter (a) $\eta$ and (b) $\epsilon$.

In case of the HQG the Rabi oscillation is performed between the ground $|0\rangle$ and bright state $|b\rangle=\gamma_{-}^{*}|-\rangle+\gamma_{+}^{*}|+\rangle$, such that the observed generalized Rabi frequency and tilt angle depend on the combination of all four detuning values.

In contrast, the gate modalities featuring a strong asymmetry with respect to $\eta$ and $\epsilon$ are concatenated out of two to four pulses. The performance of the first pulse defines the input state of the subsequent pulses. Depending on the sign of the detuning the output of the first operation changes. Hence, for different sign detunings the subsequent application of erroneous gate operations does in general not result in output states with same distance to the anticipated final state. Consequentially, no symmetry with respect to $\eta$ and $\epsilon$ is expected. Note, the SAGQG Pauli-X gate features an asymmetry as well, even though it is less pronounced than for the other modalities.

\subsection{Experimental detuning analysis}

In order to obtain an easier access to the complex robustness data the limiting cases are analysed, where only one of the parameter is assumed to be error-prone $(\epsilon=0$ or $\eta=0)$. The numeric prediction are confirmed experimentally by performing standard QPT measurements of intentionally detuned QGs (Sec. 9.1.5).

The utilization of an imperfect experimental system to measure the response to experimental detunings requires a high degree of stability and precise knowledge on the system properties. By closely monitoring and frequent retuning of the resonance $\omega_{0}$ and Rabi frequency $\Omega_{0}$ a standard deviation of $\eta$ and $\epsilon$ in the range of a few kilohertz $\left(\sigma_{\eta} \leq 50 \mathrm{kHz}\right.$ and $\left.\sigma_{\epsilon} \leq 10 \mathrm{kHz}\right)$ is ensured (Sec. A.3).

Note, the generalized Rabi frequency $\Omega^{\prime}=\sqrt{\Omega^{2}+\Delta^{2}}$ is a quantity describing the flopping of the qubit population $\left(p_{2}(t)=\Omega^{2} / \Omega^{\prime 2} \sin \left(\Omega^{\prime} \cdot t / 2\right)\right)$ and is an implication of the detuned Hamiltonian. For a detuning $\Delta$ the applied Rabi frequency $\Omega$ appearing in the Hamiltonian remains unchanged, since it defines the driving field amplitude. Therefore, fidelity data $F(\epsilon, \eta)$ for $\epsilon=0$ correspond to a vertical cut through the contour plots (black lines) presented in Fig. 12.1 and Fig. 12.2.

Experimentally $\eta$ can easily be varied between $\pm 1.5 \mathrm{MHz}$ by appropriate programming of the 
a

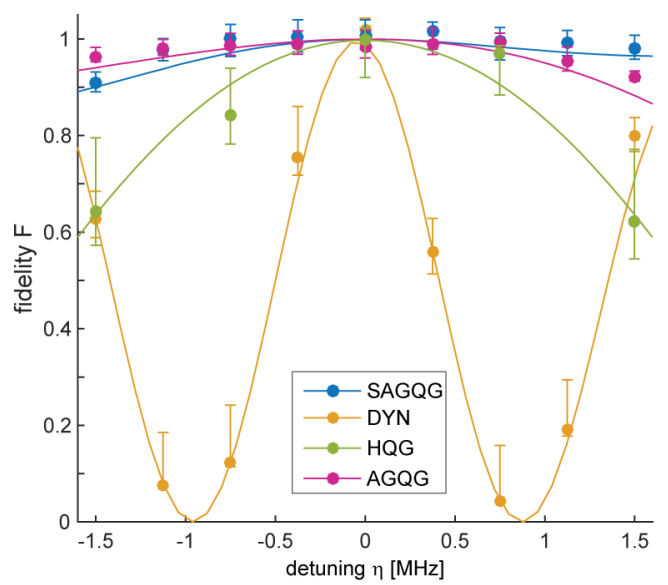

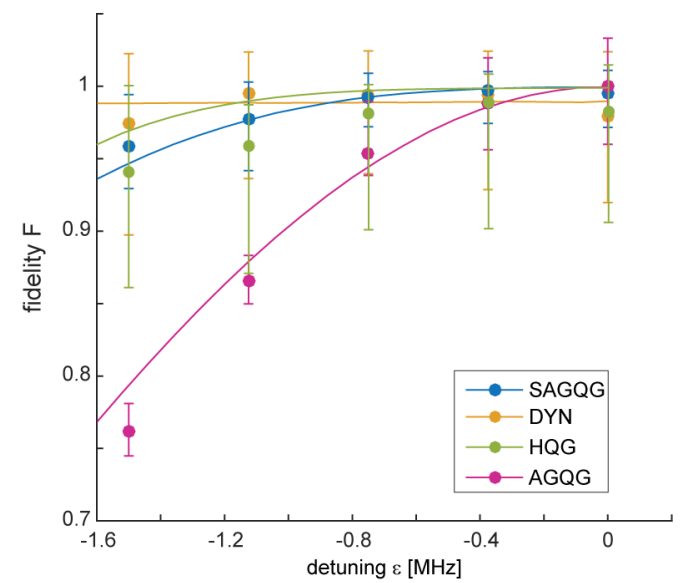

Figure 12.4.: Pauli-Z gate fidelity as a function of the detuning $\eta$ and $\epsilon$. (a) Calculated (solid lines) and measured (dots with error-bars) quantum gate fidelity of the Pauli-Z operation for dynamic (orange), Abelian geometric (pink), superadiabatic (blue) and holonomic (green) gate modality depending the detuning parameter (a) $\eta$ and (b) $\epsilon$.

AWG. However, a variation of the Rabi frequency by positive $\epsilon$ values is difficult, since the QGs are performed close to the maximal Rabi frequency $\Omega_{\max }$ by design. The presented measurements are limited to the parameter range of $-1.5 \mathrm{MHz} \leq \epsilon \leq 0 \mathrm{MHz}$. In the following the results for Pauli-X and Pauli-Z are discussed individually.

\subsubsection{Pauli-X}

Figure 12.3 presents the calculated and measured Pauli-X quantum gate fidelity as a function of the detuning (a) $\eta$ and (b) $\epsilon$ for the DYN (orange), SAGQG (blue) and HQG (green, red) gate modalities. In case of the HQG detunings of same sign $\left(\eta_{\mathrm{ss}}, \epsilon_{\mathrm{ss}}\right)$ and opposite sign $\left(\eta_{\mathrm{os}}, \epsilon_{\mathrm{os}}\right)$ but same absolute value are considered. A rotation around the $\mathrm{x}$-axis by means of the Abelian geometric quantum gate (AGQG) approach is theoretically prohibited.

Surprisingly, for large detuning parameter $\eta$ of the resonance frequency the DYN approach outperforms both geometric phase based modalities. However, it needs to be stated that the difference with respect to SAGQG is small even for unrealistically large detunings of $\pm 1.5 \mathrm{MHz}$. Both HQG $\left(\eta_{\mathrm{ss}}, \eta_{\mathrm{os}}\right)$ modalities perform poorly in this detuning regime. In the for our experimental apparatus relevant regime $|\eta| \leq 500 \mathrm{kHz}$ the fidelity of DYN and SAGQG modalities differ in the order of $10^{-3}$ at the most. In the same regime, the robustness of the $\eta_{\mathrm{os}}$ HQG can compete with the other approaches, but for driving fields with same sign detuning $\left(\eta_{\mathrm{ss}}\right)$ the fidelity is dropping quickly with $\eta$.

When considering the detuning parameter of the Rabi frequency $\epsilon$ the overall behaviour changes dramatically (Fig. 12.3.b). Again, over the whole considered parameter space the DYN modality is expected to be less sensitive to parameter mismatches than the SAGQG one. In general, the HQG is less error susceptible for the detuning parameter $\epsilon$ than for $\eta$. Depending on the chosen parameter detuning realization the HQG is either the most insensitive $\left(\epsilon_{\mathrm{ss}}\right)$ or the most error-prone $\left(\epsilon_{\mathrm{os}}\right)$ modality. 


\subsubsection{Pauli-Z}

The robustness of the Pauli-Z gate modalities with respect to the control parameter detuning $\eta$ and $\epsilon$, presented in Fig. 12.4, differ from the one of the Pauli-X gate. The dynamic realization (DYN) is sensitive to the detuning $\eta$, even dropping to zero around $\pm 1 \mathrm{MHz}$. Note, the fidelity is always well below one (Sec. 12.1.1) and shows almost no variation with $\epsilon$. The HQG is the most error prone geometric phase based gate for the detuning parameter $\eta$ but the most stable one for the detuning parameter $\epsilon$. The QG fidelity of the SAGQG in the experimental relevant regime of $\epsilon$ does not differ significantly from the one of the HQG modality but outperforms the AGQG approach substantially. Contrary, for detuning parameter $\eta$ the SAGQG and AGQG modalities are comparably robust to constant driving field parameter imperfections in the experimental relevant regime.

\subsubsection{Summary}

The performed standard QPT measurements of intentionally detuned QG operations are in good agreement with the ones obtained from numerical calculations. Results were obtained by pushing to the limits of the experimentally accessible parameter space, thereby representing a fair comparison under realistic boundary conditions. For the chosen set of QG modalities no general superior performance with respect to constant driving field parameter detunings for any of the modalities was found. Indeed, robustness strongly depends on the realized quantum gate and the most error prone detuning parameter $(\eta$ or $\epsilon$ ). In Sec. 13.2.2 the optimal choice of gate modalities is discussed in detail for the NV center in diamond and superconducting transmon qubits. The results underline the importance of the choice of quantum gate modality for any experimental implementation. A detailed analysis like the one presented here of nowadays NISQ might boost their range of applications and enables the realization of QECCs. Additional considerations on the SAGQG driving field parameter are presented in App. D.

\subsection{Average probability of error per gate}

For concatenated gate sequences realizing quantum algorithms the effects of noise and decoherence can no longer be ignored. The computational relevant average probability of error (APE) per gate is typically extracted by randomized benchmarking (RB) (Sec. 9.2). The APE combines the influence of static detuning and effects of interaction with the noise environment.

\subsubsection{Modality comparison}

As described in Sec. 9.2 randomized benchmarking analyses of the DYN, SAGQG and HQG are performed. The Abelian nature of the AGQG prohibits the realization of RB measurements purely based on this modality. In Fig. 12.5. a the average gate fidelity $f_{a}$ as a function of the number of computational gates is presented. Errors correspond to the standard error of the mean $\sigma_{\text {mean }}=\sigma / \sqrt{N}$, where $N=N_{P} \cdot N_{G}=32$ is the number of different sequences per sequence length $l_{k}$. The observed decay of the fidelity of both geometric approaches is significantly slower than for the dynamic realization. Even though, featuring the more complex amplitude, phase and frequency handling the SAGQG outperforms the HQG modality, which is requiring two driving fields. The functional defined in Eq. 9.11 is fitted to the data in order to extract the APE per gate $\epsilon_{g}$ and the combined error of initialization, readout and projection $\epsilon_{m}$. Table 12.4 summarizes the measured APE for 
Table 12.4.: Average probability of error per gate for different quantum gate modalities. APE $\epsilon_{g}$ and $\epsilon_{m}$ for purely SAGQG, DYN and HQG modalities, as well as for HQG with increased runtime and the combination of DYN .

\begin{tabular}{ccccc} 
& $\hat{x}, \hat{y}$ & $\hat{z}$ & $\epsilon_{g}\left[10^{-3}\right]$ & $\epsilon_{m}\left[10^{-3}\right]$ \\
\hline \hline I. & DYN & DYN & $23.0 \pm 7.3$ & $116.9 \pm 45.7$ \\
II. & SAGQG & SAGQG & $0.92 \pm 0.34$ & $18.0 \pm 7.8$ \\
III. & HQG & HQG & $7.39 \pm 1.69$ & $7.07 \pm 1.21$ \\
IV. & HQG $_{\text {long }}$ & HQG & $11.31 \pm 2.16$ & $12.83 \pm 21.764$ \\
V. & DYN & AGQG & $5.87 \pm 1.05$ & $28.35 \pm 12.15$ \\
VI. & SAGQG & AGQG & $9.96 \pm 2.74$ & $21.64 \pm 10.56$
\end{tabular}

randomized benchmarking purely realized by DYN, SAGQG or HQG (columns I.-III.). The value of the dynamic gate APE in the order of $10^{-2}$ is not sufficient to efficaciously implement QECCs $[20,12]$. However, under equivalent conditions the SAGQG perform significantly better with an almost two order of magnitude improved average error rate $\left(\epsilon_{g}=0.0009 \pm 0.0003\right)$. Note, the realization of the computational gates (rotations by $\pi / 2$ ) requires the application of two subsequent pulses in the HQG case, finally resulting in an APE of $\epsilon_{g}=0.0074 \pm 0.0017$.

\subsubsection{Gate duration}

The gate designs have been optimized for fast execution speed. Due to the experimental boundary conditions the HQG performs faster $\left(t_{\mathrm{Gate}}^{\mathrm{HQG}}=256 \mathrm{~ns}\right)$ than the SAGQG $\left(t_{\mathrm{Gate}}^{\mathrm{SAGQ}}=320 \mathrm{~ns}\right)$. Here, it is investigated if the SAGQG performs better than the HQG, since its gate evolution is closer to the adiabatic limit. The gate duration of the $\mathrm{HQG} t_{\mathrm{Gate}}^{\mathrm{HQG}}$ is extended to be equal to $t_{\mathrm{Gate}}^{\mathrm{SAGG}}$. In Fig. 12.5.b the RB analysis of the HQG with an extended gate duration ( $\mathrm{HQG}_{\text {long }}$, orange). Instead of a decrease of the APE an increase to $\epsilon_{g}^{\mathrm{HQG}_{\text {long }}}=0.0113 \pm 0.0022$ is observed (Tab. 12.4.III.-IV.). The result indicates that the, compared to the SAGQG, worse gate performance is not induced by high but low frequency noise. A further discussion on the effect of the noise frequency on the QG is presented in Sec. 13.2.2.

\subsubsection{Combining modalities}

The analysis in Sec. 12.4 suggest that the pure dynamic set of gates is susceptible for errors and results in a poor APE in the RB analysis. A significant improvement can be obtained when replacing the dynamic phase-shift $\left(R_{z}(\theta)\right)$ gate by its more robust AGQG counterpart (violet, Fig. 12.5.c). In this way the APE could be decreased from $\epsilon_{g}=0.0230 \pm 0.0073$ to $\epsilon_{g}=0.0059 \pm$ 0.0011 , revealing the potential power of the combination of different gate modalities.

Similarly, the randomized benchmarking of a set realized by AGQG $\left(R_{z}(\theta)\right)$ and SAGQG $\left(\left(R_{x}(\theta)\right.\right.$ and $R_{y}(\theta)$ ) was performed (red). In this composition the APE increased, compared to the pure SAGQG realization, to $\epsilon_{g}=0.0083 \pm 0.0022$. For the in Sec. 12.6.2 determined most likely source of error $\epsilon$ a similar robustness is expected in the experimental relevant regime. However, the observed decrease of the APE is a consequence of the different required driving field frequency correction factors (Sec. 10.1.2). The effect was not observed for the combination of DYN and AGQG gates, since both modalities are based on simple $\pi$-pulses. Note, this source of error can be suppressed by the development of microwave components with homogeneous frequency response at the transition frequencies. 


\subsection{Summary}

In this chapter the robustness of various quantum gate modalities with respect to constant control parameter imperfections was calculated and confirmed experimentally. The direct comparison of dynamic and geometric phase based quantum gates revealed a surprising competitiveness of the dynamic Pauli-X gate with respect to this kind of error. The most robust performance depends on the realized gate, the most error-prone control parameter and in case of the HQG on the sign of the parameter variation. In conclusion, when searching the most stable universal set of quantum gates a combination of different quantum gate modalities should be considered. The validity of this concept was confirmed by a randomized benchmarking analysis. 
a

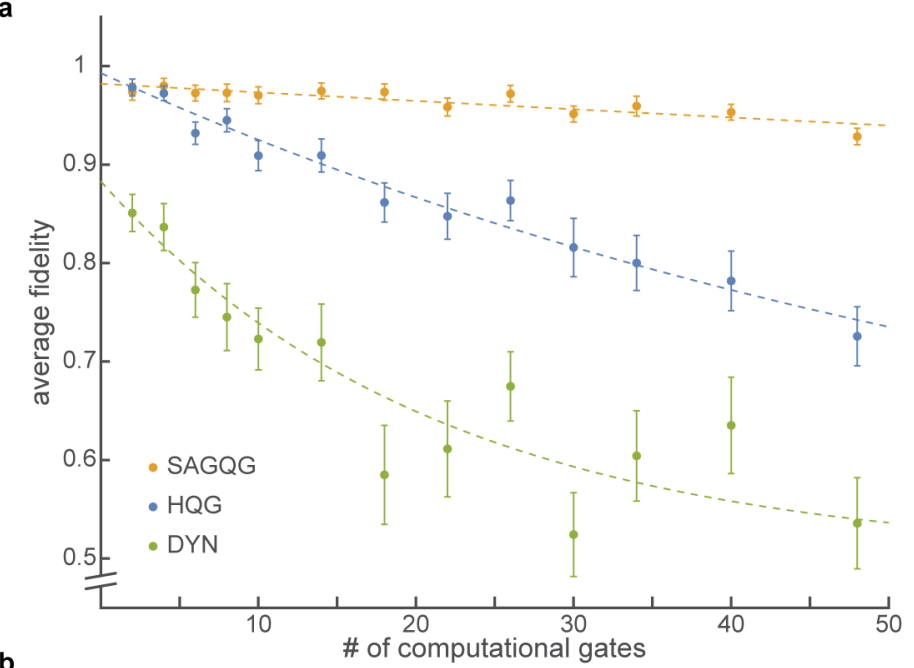

b

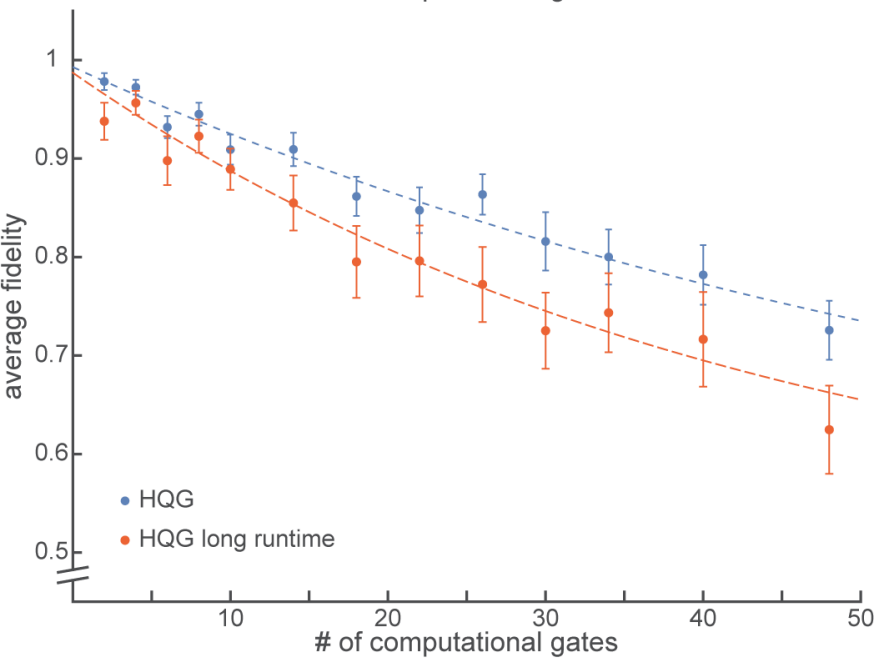

c

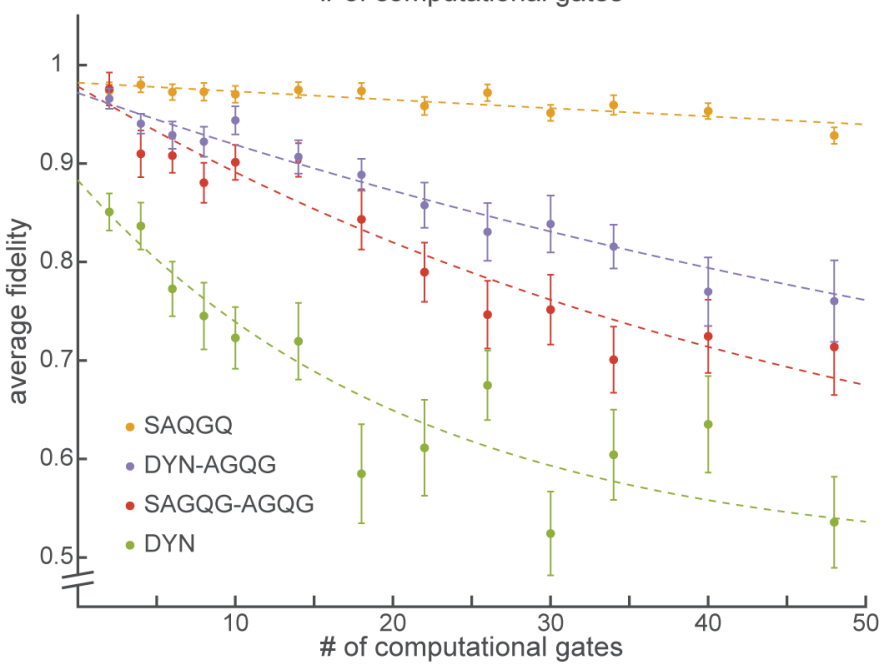

Figure 12.5.: Randomized benchmarking measurement. Average gate fidelity as a function of the number of computational gates $l$ for: (a) pure DYN (green), SAGQG (yellow) and HQG (blue) quantum gates, (b) HQG (blue) and HQG with increased gate duration (orange), as well as (c) DYN (green), SAGQG (orange), combined DYN-AGQG (violet) and combined SAGQG-AGQG (red). Error bars represent the standard error of the mean. Dashed lines represent a fit of the function $f(l)=1-\left(\left(1-\alpha_{n} \epsilon_{m}\right)\left(1-\alpha_{n} \epsilon_{g}\right)^{l}+1\right) / \alpha_{n}$, with $\alpha_{n}=2$, respectively. 

Discussion 



\section{Conclusion and outlook}

Within this thesis two key challenges in the field of quantum information processing (QIP) have been addressed. STED microscopy was applied in order to identify promising candidates for solidstate spin based QIP applications and to determine properties to increase their production yield respectively (Sec. 13.1). In the second part the implementation and benchmarking of high-fidelity quantum gates for fault-tolerant quantum computing was conducted in Sec. 13.2.

\subsection{Super-resolution microscopy of solid-state spins}

In the scope of this thesis STED imaging of ST1 centers and NV center based fluorescent nuclear track detectors (FNTDs) has been demonstrated. The first represents an important step in the screening process for defect centers suitable for solid-state spin based QIP applications (Sec. 13.1.1). The second makes it possible to determine the vacancy diffusion coefficient in diamond precisely and offers the potential for new insights in radiation treatment (Sec. 13.1.2).

\subsubsection{ST1 center imaging}

In Ch. 6, STED imaging of the ST1 center in diamond was performed. Besides to the NV center the ST1 center represents the only known defect center in diamond to feature optically detected magnetic resonances (ODMRs) at room temperature [77, 109]. Its nuclear spin free constituent makes the ST1 center an interesting candidate for QIP and QS applications. In order to provide an efficient dipole-dipole coupling between neighboring ST1 center electron spins, a separation of the order of $30 \mathrm{~nm}$ is fundamentally required. Hence, the optical spin-state read-out of a ST1 center based quantum register needs to be performed by a super-resolution imaging technique. In Sec. 6.2.1, it was shown that the STED microscope can be used for super-resolution imaging of NV centers and ST1 centers with similar performance, due to their similar optical properties. This result suggests that optical resolutions down to a few nanometers are possible [43]. Screening for the constituent of the ST1 center and other defect centers featuring ODMR is currently ongoing $[76]$.

\subsubsection{Fluorescent nuclear track detection}

Super-resolution STED microscopy of diamond based FNTDs has been demonstrated in Ch. 7 . Localization algorithms revealed a lateral substructure of the NV center distribution, which was blurred in confocal microscopy (Sec. 7.2.3). Additionally, sub-cascade events could be resolved (Sec. 7.2.1), enabling the detailed observation of the absorption process of ionizing radiation in matter.

The established aluminium-oxide FNTDs are known to suffer from strong and inhomogeneous background [96]. Particularly, in STED microscopy high intensities in combination with a reduced fluorescence signal cause a loss in the signal-to-background ratio. As a consequence, the optical resolution is limited to roughly $100 \mathrm{~nm}$. 
An alternative approach is the direct imaging of fhe fluorescent isolated vacancies ("general radiation1" (GR1) center) in diamond [170]. GR1 centers feature a zero phonon line (ZPL) at $742 \mathrm{~nm}$ and an emission sideband extending to the near-infrared. The direct imaging of GR1 centers generated by ionizing radiation would allow their utilization as FNTD without any additional treatment. The resolution is ultimately limited by the initial vacancy distribution known to be on the order of $5 \mathrm{~nm}$ [75]. However, the radiative lifetime of approx. $100 \mathrm{~ns}$ is effectively reduced by non-radiative decay channels, resulting in an overall fluorescence lifetime on the order of $2 \mathrm{~ns}$ [22]. Additionally, the strong non-radiative decay channels cause a low brightness of the GR1 center. So far, neither super-resolution imaging nor imaging of single GR1 centers has been reported.

Currently, the demonstrated STED imaging of NV center based FNTDs represents the most favourable platform for solid-state nuclear track detectors (SSNTDs). It offers a mechanism for the determination of the vacancy diffusion coefficient in diamond with high precision. This knowledge will potentially lead to optimized annealing protocols and hence to the deterministic generation of NV centers. Additionally, by visualizing the absorption of ionizing radiation in matter, new insights in radiation treatment might be obtained.

\subsection{High-fidelity quantum computation}

The implementation of quantum error correction codes (QECCs) for the realization of fault-tolerant quantum computers requires high-fidelity quantum operations (Sec. 1.3). In the scope of this thesis a conceptually new non-Abelian, non-adiabatic geometric QGs modality has been realized. In the following section, these results are summed up and a qualitative comparison to QGs realized by composite pulse sequences is performed (Sec. 13.2.1). A comparative benchmarking analysis of different quantum gate modalities has been conducted in order to identify the most robust universal set of single-qubit quantum gates for the considered experimental system (Ch. 12). Here, the possible impact in the field of QIP is discussed.

\subsubsection{Superadiabatic geometric quantum gates}

In Ch. 11 the superadiabatic geometric quantum gate (SAGQG) [81] was realized with close to ideal fidelity for single-qubit operations on the NV center electron spin under ambient conditions. The implementation of the likewise proposed two-qubit controlled-NOT gate was not possible due to the lack of suitable diamond samples.

SAGQGs exploit the concept of transitionless quantum driving (TQD) [150] in order to overcome the adiabatic requirement for the occurrence of the Berry phase [78]. A minimal gate execution time equivalent to the duration of four $\pi$-pulses at the system's maximal Rabi frequency was theoretically derived (Sec. 11.4) and experimentally confirmed (Sec. 11.8). It needs to be stressed that this limit is a consequence of the experimentally accessible parameter space, rather than theoretical requirements like the need for adiabaticity. Nevertheless, the gate duration is comparable to dynamic pulses (Tab. 12.2) and shorter than the one of direct current (DC) noise resilient composite pulse sequences. Composite pulse sequences (e.g., BB1 and SUPCODE) have shown remarkable quantum gate fidelities utilizing the NV center electron spin. But these realizations required tremendous experimental efforts in terms of microwave pulse characterization and error compensation of the driving field [171]. In contrast, geometric phase based QG realizations like the SAGQG feature an intrinsic robustness with respect to local (high frequency) noise [132]. A further enhancement of the SAGQG fidelity might be achieved by reducing the DC noise introduced by 
the system's frequency response (Sec. 10.1.2).

Even though it was proposed and demonstrated on the NV center electron spin, the gate modality can be performed on any experimental platform featuring a sufficient control of the driving field degrees of freedom. A precise time-dependent phase, amplitude and frequency handling of the driving field is required, representing a potential challenge in optically driven systems (e.g., [128, 129]). The set of non-Abelian non-adiabatic geometric quantum gates realized on a simple two-level system by a single driving field represents a high-fidelity universal set of QGs implemented with minimal experimental resources. In contrast, the non-adiabatic HQG featuring a three-level system and two driving fields (Sec. 8.6) sets additional requirements on the physical system (e.g., driving field bandwidth) and causes a substantial time-overhead in the characterization measurements.

The non-Abelian nature of the SAGQG is obtained by the evolution of a pair of orthogonal cyclic states as proposed by S.-L. Zhu and Z.D. Wang (ZW, Sec. 11.3.1) [160]. In this approach the cyclic states of the system are dark states and hence, by design, no dynamic phase is acquired. Recently, it was shown that the non-adiabatic HQG (Sec. 8.6) can be interpreted as a ZW gate as well [172], underlining the importance of this physical concept.

\subsubsection{Benchmarking analysis}

The development of fault-tolerant quantum computers requires the identification of the most reliable out of various promising physical systems. Initial comparative benchmarking analyses of different platforms are currently ongoing. For example, an experimental comparison of a 5-qubit quantum register realized by the IBM quantum computer and a trapped ion system has been performed [173]. Additionally, the effect of control noise has been investigated theoretically for different quantum dot systems [174]. However, in order for this kind of benchmarking to be conclusive, it needs to be ensured that the compared physical platforms are operated at their optimal configuration with maximum robustness. Hence, the identification of the most reliable universal set of quantum gates for the individual platforms would represent the first step towards a more "universal" performance evaluation of different quantum systems.

For a set of single-qubit quantum gates, a quantitative performance analysis comparing simple dynamic (DYN), Abelian geometric (AGQG), superadiabatic geometric (SAGQG) and nonadiabatic holonomic (HQG) quantum gates has been conducted (Ch. 12). Standard quantum process tomography (QPT) confirmed the high fidelity implementation of all considered quantum gate modalities (Sec. 12.3). In fact, the obtained fidelities exceed the threshold where distinction is prohibited due to experimental imperfections of the QPT itself. Subsequently, the effect of DC noise on the QG fidelity has been calculated numerically and measured experimentally (Sec. 12.4 and Sec. 12.5). Additionally, conclusions regarding the contribution of high frequency noise could be drawn by combining randomized benchmarking data and knowledge of the properties of geometric phases (Sec. 12.6). Here, the effects of the different noise frequencies are interpreted in depth, before performing case studies on two of the most promising experimental platforms for QIP.

\section{Constant driving field imperfections}

The robustness with respect to constant driving field parameter imperfections was investigated numerically (Sec. 12.4) and experimentally (Sec. 12.5) under realistic experimental boundary conditions (Sec. 12.2). This kind of error might be interpreted as DC or gate duration $\left(t_{\text {Gate }}\right)$ dependent 
low frequency noise, including frequencies up to:

$$
f_{\mathrm{DC}} \ll \frac{1}{10 \cdot t_{\mathrm{Gate}}} .
$$

Fluctuations at a rate below $f_{\mathrm{DC}}$ are interpreted as constant and hence define the values of the detuning parameters $\eta$ and $\epsilon$ of the realized gate (Sec. 12.4). Typically, for the NV center $f_{\mathrm{DC}}$ is on the order of several hundred kilohertz. The exact value of $\eta$ and $\epsilon$ might vary in subsequent gates of an algorithm, such that the amplitude of the low frequency noise defines the standard deviations $\sigma_{\eta}$ and $\sigma_{\epsilon}$. Depending on the realized QG and chosen modality, the robustness with respect low frequency noise varies significantly (Sec. 12.4).

The high-fidelity implementation of a universal set of single-qubit QGs by purely dynamic gate operations appears unlikely due to the non-trivial realization of phase-shift gates. However, when combining dynamic rotations around the $\hat{x}$ and $\hat{y}$-axis $\left(R_{x}(\theta)\right.$ and $\left.R_{y}(\theta)\right)$ with a simple geometric Abelian phases-shift gate, the average probability of error (APE) per gate is reduced considerably (Sec. 12.6.3). This is achieved by replacing the most error-prone gate operation with respect to $f_{\mathrm{DC}}$ by a more robust one. The example illustrates that exploiting different theoretical concepts is a valid tool for the realization of universal high-fidelity quantum operations.

A superior robustness of the SAGQG in comparison to the HQG for constant driving field imperfections, as predicted in by Liang et al. [81], could in general not be confirmed (Sec. 12.4.3). While in [81] only the single-qubit phase shift gate, requiring a single driving field in the nonadiabatic holonomic case, is considered here, a more complete analysis including the general case of quantum gates realized by two driving fields was performed. In this situation the exact robustness depends strongly on the assumed detunings $\eta$ and $\epsilon$ for the individual transitions $|0\rangle \leftrightarrow|-\rangle$ and $|0\rangle \leftrightarrow|+\rangle$ (Fig. 12.2). A detailed case study considering specific properties of the physical platform for NV centers and superconducting transmon qubits is presented at a later point of the discussion.

\section{Suppression of high frequency noise}

It was shown that adiabatic geometric phase based quantum gates are resilient against high frequency noise $[132,133]$. As indicated in [175] this intrinsic robustness holds true for SAGQGs and non-adiabatic HQGs. A rough estimate of the high frequency threshold $f_{\mathrm{HF}}$, defining the noise frequency which is efficiently suppressed by the geometric property of the quantum gate, might be obtained by:

$$
f_{\mathrm{HF}} \gg \frac{10}{t_{\mathrm{Gate}}} .
$$

The utilization of non-adiabatic quantum gates pushes the efficiently suppressed noise frequency $f_{\mathrm{HF}}$ towards higher values. Hence, it needs to be re-evaluated which frequencies can be considered as high. At non-adiabatic gate durations in the order of $t_{\text {Gate }} \approx 300 \mathrm{~ns}$ (Tab. 12.2) the threshold frequency is expected at $f_{\mathrm{HF}} \sim 33 \mathrm{GHz}$. This value exceeds any reasonable assumption for the spin-noise environment in diamond, which is typically featuring a DC-centered Lorentzian spectral noise density (e.g., [42, 166]). Hence, non-adiabatic geometric phase based QGs are limited in the ability to suppress environmentally induced fluctuations. This conclusion is supported by the RB measurement presented in Sec. 12.6.2, where an increase of the HQG gate duration from $t_{\text {Gate }}=256 \mathrm{~ns}$ to $t_{\mathrm{Gate}}=320 \mathrm{~ns}$, corresponding to a decrease of $f_{\mathrm{HF}} \approx 39 \mathrm{GHz}$ to $f_{\mathrm{HF}} \approx 31 \mathrm{GHz}$ and an increase of $f_{\mathrm{DC}} \approx 390 \mathrm{kHz}$ to $f_{\mathrm{DC}} \approx 312 \mathrm{kHz}$, caused an increase of the APE.

However, driving field fluctuations due to the microwave amplifier noise and rise time are able to 
a

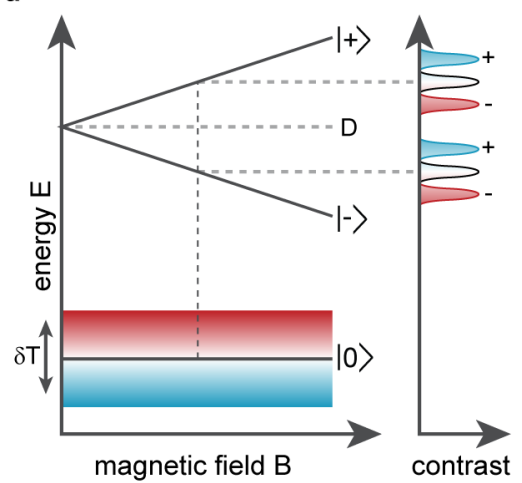

b

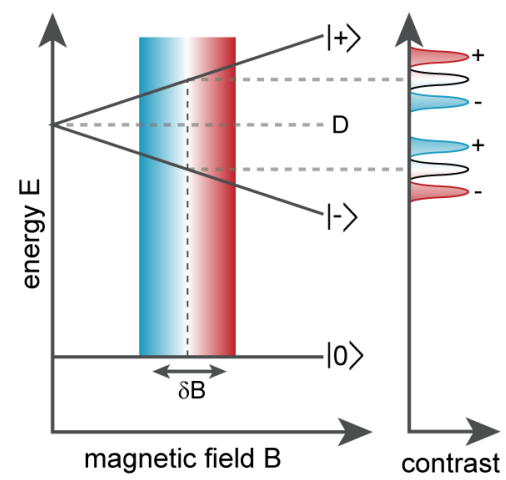

Figure 13.1.: Possible sources of constant errors. (a) Energy scheme of the NV center triplet ground state. $D$ depends on temperature fluctuations $\delta T$. The energy spectra on the right side illustrate the resulting same sign detuning from the original resonances (black). (b) Random magnetic field fluctuations $\delta B$ change the absolute value but not the sign of the Zeeman term. Consequently, the energy spectra feature an opposite sign detuning from the original resonances (black).

occur in the frequency regime above $f_{\mathrm{HF}}$. One concludes therefore, that non-adiabatic geometric phase based quantum gates are accessible to environment induced intermediate frequency noise but resilient against control parameter noise. In diamond the contribution intermediate frequency noise can be efficiently suppressed by the use of optimized samples. The robustness with respect to DC noise is comparable to that of the dynamic realizations, and supremacy depends on the exact QG modality.

\section{Case studies}

In the following discussion, the optimal choice of a set of universal single-qubit QGs for the physical platform of NV centers in diamond and superconducting transmon qubits are discussed. While the first was investigated in the scope of the thesis, the later represents the system currently featuring the largest number of physical quantum bits, making it one of the most promising candidates for near-term, scalable, universal quantum computation.

\section{NV centers in diamond}

For the NV center, the precise knowledge of the main sources of error within the experimental apparatus represents the basis for the discussion. First, the effect of DC noise on the system's Hamiltonian $H$ (Eq. 2.1) is considered. The two possible sources of error are drifts of the electron spin resonance (ESR) due to changes of the zero field splitting $D$, and random magnetic field drifts $\delta B$. The effects of the respective noise on the magnetic field dependent energy levels $(|0\rangle,|-\rangle$ and $|+\rangle)$ of $H$ are illustrated in Fig. 13.1. A change of the temperature by $\delta T$ varies $D$ and both transitions are shifted symmetrically (a). Hence, temperature induces a same sign detuning $\eta_{\mathrm{ss}}$ with respect to the ESR resonance. In contrast, drifts of the magnetic field by $\delta B$ change the Zeeman term asymmetrically (b). Consequentially, the transitions $|-\rangle$ and $|+\rangle$ suffer an opposite sign detuning $\eta_{\mathrm{os}}$.

In Sec. A.3 long-term measurements of the resonance frequency $\omega_{0}$, the Rabi frequency $\Omega_{0}$ and the temperature are presented. The resonance frequency $\omega_{0}$ shows fluctuations correlated with the ambient temperature. However, the observed drifts of $\omega_{0}$ are larger than the theoretically expected value of $\frac{d D}{d T}=-77 \mathrm{kHz} \mathrm{K}^{-1}[176]$. Thermal expansion of the magnet mounting system appears to 
be responsible for magnetic field fluctuations $\delta B$ in the order of $0.1 \mathrm{G}$. The Rabi frequency remains within our measurement precision constant during the whole measurement interval. Small drifts of the Rabi frequency originate from fluctuations of the driving field amplitude or movements of the microwave antenna and are expected to be of same sign $\left(\epsilon_{\mathrm{ss}}\right)$ for both transitions. Hence, for the holonomic Pauli-X gate the most likely detuning parameter combination is $\eta_{\mathrm{os}} \epsilon_{\mathrm{ss}}$ for the NV center in diamond. At the same time for $\eta_{\mathrm{os}} \epsilon_{\mathrm{SS}}$ the highest robustness of the non-adiabatic HQG Pauli-X gate is expected. Even though two detuned driving fields are involved, in this configuration the HQG provides the most stable realization with respect to the detuning $\epsilon$ and competing robustness with respect to detuning $\eta$. For realizations of the Pauli-Z gate the HQG realization appears to be sensitive to the detuning $\eta$ and superior performance is expected for the AGQG and SAGQG modalities. In conclusion, the benchmark analysis suggests that the SAGQG modality to represents the most robust set of quantum gates, which is in agreement with our RB measurements (Sec. 12.6).

We note that, the set consisting of SAGQG $\left(R_{z}(\theta)\right)$ and HQG $\left(R_{x}(\theta)\right.$ and $\left.R_{y}(\theta)\right)$ might also result in a high-fidelity realization. However, a combination of HQG and the SAGQG gate modalities requires an additional projection between the involved computational subspaces $(\{|0\rangle,|-\rangle\}$ and $\{|-\rangle,|+\rangle\})$, which is likely to compensate potential advantages of the combination of the different gate modalities.

\section{Superconducting transmon qubits}

Nowadays, the most scaled, circuit based, noisy intermediate scale quantum computers (NISQs) are based on superconducting transmon qubits [8, 9]. Transmon qubits feature, depending on the experimental realization, transition frequencies $\omega_{0}$ in the order of several gigahertz, while Rabi frequencies $\Omega_{0}$ of up to tens of megahertz have been obtained [177, 178]. Hence, for first qualitative conclusions the calculated data presented in Sec. 12.4.3 might be used, but for a quantitative robustness estimation the numerical calculation needs to be repeated with the exact parameter values of the considered transmon qubit.

The spectral noise density was measured to decay with $1 / f^{\alpha}$, where $\alpha \approx 0.6$ is constant [179]. In general, compared to the NV center, faster gate operation increases the frequency threshold $f_{\mathrm{HF}}$ even further. At the same time, noise frequencies which can be counted as DC contribution $\left(f_{\mathrm{DC}}\right)$ are increased. Consequentially, the focus of the benchmark analysis is on the robustness with respect to constant detuning parameters $\eta$ and $\epsilon$. The anharmonicity of the system causes leakage to neighboring transitions when driving a specific one, and an unintentionally increased Rabi frequency is expected [180, 181]. Temperature fluctuations are strongly suppressed by stateof-the-art cryostats. For this reason, the most likely error is a same sign detuning of $\epsilon$ towards positive values $\left(\epsilon_{\mathrm{Ss}}\right)$. In this configuration a universal set of QGs consisting solely of HQGs appears to be remarkably robust (Sec. 12.4), significantly outperforming the dynamic approach. Still, for positive $\epsilon$ the SAGQG Pauli-Z is expected to be the most robust implementation. Due to the required projection of the different computational subspaces when combining SAGQG and HQG, for superconducting transmon qubits a purely HQG realization is suggested.

\subsection{Concluding remarks}

In the last decade, the number of quantum bits within several quantum register realizations has been continuously increasing. Hence, scalability, even though not yet achieved for every physi- 
cal system, no longer appears to be the limiting factor for fault-tolerant quantum computation. Instead, the finite quantum gate fidelity prevents the efficient realization of quantum error correction codes. In this thesis, a new approach for geometric phase based quantum gates was realized with a fidelity beyond the required error threshold. Additionally, a standardized benchmarking routine for the identification of the most robust universal set of quantum gates for a given physical system is suggested. This set of gates is not necessarily realized by a single gate modality and might vary with the physical platform. The proposed routine was demonstrated for the NV center in diamond but is of general validity. A systematic application of the benchmarking analysis to noisy intermediate scale quantum computers will pave the way towards the realization of fault-tolerant quantum computation. 



\section{Bibliography}

[1] R.P. Feynman. "Simulating Physics with Computers". In: Int. J. Theor. Phys. 21.6 (1982), pp. 467-488. DOI: 10.1007/BF02650179.

[2] D. Deutsch. "Quantum theory, the Church-Turing principle and the universal quantum computer". In: Proc. Royal Soc. Lond. A 400 (1818 1985), pp. 97-117. DOI: 10.1098/rspa. 1985.0070 .

[3] J. Preskill. "Quantum computing and the entangelement frontier". arXiv:1203.5813. 2012.

[4] P.W. Shor. "Polynomial-Time Algorithms for Prime Factorization and Discrete Logarithms on a Quantum Computer". In: SIAM J. Comput. 26 (5 1997), pp. 1484-1509. DOI: 10. $1137 /$ S0097539795293172.

[5] L.K. Grover. "Quantum Mechanics Helps in Searching for a Needle in a Haystack". In: Phys. Rev. Lett. 79.2 (2 1997), pp. 325-328. DOI: 10.1103/PhysRevLett.79.325.

[6] H.R. Moser. "The quantum mechanical solution of the travelling salesman problem". In: Physica E 16 (2 2003), pp. 280-285. DOI: 10.1016/S1386-9477(02)00928-1.

[7] D-Wave Announces D-Wave 2000Q Quantum Computer and First System Order. Tech. rep. D-Wave System Inc., 2017.

[8] C. Vu. IBM announces advances to IBM quantum systems $\& 3$ ecosystem. Tech. rep. IBM, 2017.

[9] J. Kelly. A preview of bristleonce, google's new quantum processor. Tech. rep. Quantum AI Lab, 2018.

[10] J. Preskill. "Quantum computing in the NISQ era and beyond". arXiv:1801.00862v2. 2018.

[11] J. Preskill. "Reliable quantum computers". In: Proc. R. Soc. Lond. A 454 (1969 1998), pp. 385-410. DOI: 10.1098/rspa.1998.0167.

[12] E. Knill. "Quantum computing with realistically noisy devices". In: Nature 434 (2005), pp. 39-44. DOI: 10.1038/nature03350.

[13] W.K. Wootters and W.H. Zurek. "A single quantum cannot be cloned". In: Nature 299 (1982), pp. 802-803. DOI: 10.1038/299802a0.

[14] D. Dieks. "Communication by EPR devices". In: Phys. Lett. A 92.6 (1982), pp. 271-272. DOI: $10.1016 / 0375-9601(82) 90084-6$.

[15] W. Pauli. "Zur quantenmechanik des magentischen elektrons". In: Z. Physik 43 (9-10 1927), pp. 601-623. DOI: 10.1007/BF01397326.

[16] M.A. Nielsen and I.L. Chuang. Quantum Computation and Quantum Information. Cambridge: Cambridge University Press, 2005.

[17] A. Barenco et al. "Elementary gates for quantum computation". In: Phys. Rev. A 52 (5 1995), pp. 3457-3467. DOI: 10.1103/PhysRevA.52.3457. 
[18] D.P. DiVincenzo. "Two-bit gates are universal for quantum computation". In: Phys. Rev. A 51.2 (1995), pp. 1015-1022. DOI: 10.1103/PhysRevA.51.1015.

[19] T.D. Ladd et al. "Quantum computers". In: Nature 464.4 (2010), pp. 45-53. DOI: 10.1038/ nature08812.

[20] P. W. Shor. "Scheme for reducing decoherence in quantum computer memory". In: Phys. Rev. A 52.4 (1995). DOI: 10.1103/PhysRevA.52.R2493.

[21] X. Rong et al. "Experimental fault-tolerant universal quantum gates with sold-state spins under ambient conditions". In: Nat. Commun. 6.8748 (2015). DOI: 10.1038/ncomms9748.

[22] F. Jelezko and J. Wrachtrup. "Single defect centres in diamond: a review". In: Phys. Stat. Sol. (a) 203.13 (2006). DOI: 10.1002/pssa. 200671403.

[23] J.E. Butler, Y.A. Mankelevich, A. Cheesman, J. Ma, and M.N.R. Ashfold. "Understanding the chemical vapor deposition of diamond: recent progress". In: J. Phys.: Condens. Matter 21.364201 (2009). DOI: $10.1088 / 0953-8984 / 21 / 36 / 364201$.

[24] G. Balasubramanian et al. "Ultralong spin coherence time in isotopically engineered diamond". In: Nat. Mater. 8 (2009), pp. 3383-387. DOI: 10.1038/nmat2420.

[25] T.M. Babinec et al. "A diamond nanowire single-photon source". In: Nat. Nanotechnol. 5 (2010), pp. 195-199. DOI: 10.1038/nnano.2010.6.

[26] V.S. Ilchenko et al. "Whispering gallery mode diamond resonator". In: Opt. Lett. 38.21 (2013). DOI: $10.1364 / 0 \mathrm{~L} .38 .004320$.

[27] P. Maletinsky et al. "A robust scaning diamond sensor for nanoscale imaging with single nitrogen-vacancy centers". In: Nat. Nanotechnol. 7 (2012), pp. 320-324. DOI: 10 . 1038 / nnano.2012.50.

[28] J.P. Hadden et al. "Strongly enhanced photon collection from diamond defect centers under microfabrication integrated solid immersion lenses". In: App. Phys. Lett. 97.241901 (2010). DOI: $10.1063 / 1.3519847$.

[29] N. Aslam, G. Waldherr, P. Neumann, F. Jelezko, and J. Wrachtrup. "Photo-induced ionization dynamics of the nitrogen vacancy defect in diamond investigated by single-shot charge state detection". In: New. J. Phys. 15.013064 (2013). DOI: 10.1088/1367-2630/15/1/ 013064.

[30] A.T. Collins, M.F. Thoaz, and M.I.B. Jorge. "Luminescence decay time of the $1.945 \mathrm{eV}$ centre in type 1b diamond". In: J. Phys. C: Solid State Phys. 16.11 (1983). DoI: 10.1088/ 0022-3719/16/11/020.

[31] A. Gruber et al. "Scanning Confocal Optical Microscopy and Magnetic Resonance on Single Defect Centers". In: Science 276 (1997). DOI: 10.1126/science.276.5321.2012.

[32] E. Rittweger, K.Y. Han, S.E. Irvine, C. Eggeling, and S.W. Hell. "STED microscopy reveals crystal colour centres with nanometric resolution". In: Nat. Photonics 3 (2009), pp. 144147. DOI: $10.1038 /$ nphoton.2009.2.

[33] N.B. Manson and J.P. Harrison. "Photo-ionization of the nitrogen-vacancy center in diamond". In: Diam. Relat. Mater. 14 (10 2005), pp. 1705-1710. DOI: 10.1016/j.diamond. 2005.06 .027$.

[34] M. W. Doherty et al. "The nitrogen-vacancy colour centre in diamond". In: Phys. Rep. 528.1 (2013). DOI: $10.1016 / j \cdot$ physrep.2013.02.001. 
[35] T. Gaebel et al. "Room-temperature coherent coupling of single spins in diamond". In: Nature 2 (2006), pp. 408-413. DOI: 10.1038/nphys318.

[36] J. Harrison, M.J. Sellars, and N.B. Manson. "Optical spin polarization of the N-V centre in diamond". In: JOL 107.1-4 (2004), pp. 245-248. DOI: 10.1016/j.jlumin.2003.12.020.

[37] M. V. Gurudev Dutt et al. "Quantum Register Based on Individual Electronic and Nuclear Spin Qubits in Diamond". In: Science 316 (2007), pp. 1312-1316. DOI: 10.1126/science. 1139831.

[38] D.A. Redman, S. Brown, R.H. Sands, and S.C. Rand. "Spin dynamics and electronic states of N-V centers in diamond by EPR and four-wave-mixing spectroscopy". In: Physical Review Letters 67.24 (1991). DOI: 10.1103/PhysRevLett.67.3420.

[39] B. Smeltzer, J. McIntyre, and L. Childress. "Robust control of individual nuclear spins in diamond". In: Phys. Rev. A 80.050302(R) (2009). DOI: 10.1103/PhysRevA.80.050302.

[40] V. Jacques et al. "Dynamic Polarization of Single Nuclear Spins by Optical Pumping of Nitrogen-Vacancy Color Centers in Diamond at Room Temperature". In: Phys. Rev. Lett. 102.057403 (2009). DOI: 10.1103/PhysRevLett.102.057403.

[41] F. Jelezko, T. Gaebel, I. Popa, A. Gruber, and J. Wrachtrup. "Observation of coherent oscillation in a single electron spin". In: Phys. Rev. Lett. 92.076401 (7 2004). DOI: 10.1103/ PhysRevLett.92.076401.

[42] N. Bar-Gill, L.M. Pham, A. Jarmola, D. Budker, and R.L. Walsworth. "Solid-state spin electronic spin coherence time approaching one second". In: Nat. Commun. 4.1743 (2012). DOI: $10.1038 /$ ncomms 2771 .

[43] D. Wildanger et al. "Solid Immersion Facilitates Fluorescence Microscopy with Nanometer Resolution and Sub-Ångström Emitter Localization”. In: Adv. Mater. 24.44 (2012), pp. 309313. DOI: 10.1002/adma. 201203033.

[44] E. Rittweger, D. Wildanger, and S.W. Hell. "Far-field fluorescence nanoscopy of diamond color centers by ground state depletion". In: EPL 86.14001 (2009). DOI: 10.1209/02955075/86/140010.

[45] K.Y. Han, S.K. Kim, C. Eggeling, and S.W. Hell. "Metastable Dark States Enable Ground State Depletion Microscopy of Nitrogen Vacancy Centers in Diamond with DiffractionUnlimited Resolution". In: Nano. Lett. 10.8 (2010). DOI: 10.1021/nl102156m.

[46] P. C. Maurer et al. "Far-field optical imaging and manipulation of individual spins with nanoscale resolution". In: Nat. Physics 6 (2010), pp. 912-918. DOI: 10.1038/nphys1774.

[47] J.-C. Jskula et al. "Superresolution optical magnetic imaging and spectroscopy using individual electronic spins in diamond". In: Opt. Express 25.10 (2017), pp. 11048-11064. DOI: 10.1364/OE.25.011048.

[48] X. Chen et al. "Subdiffraction optical manipulation of the charge state of nitrogen vacancy center in diamond". In: Light Sci. Appl. $4 . \mathrm{e} 230$ (2015). DOI: 10.1038/1sa.2015.3.

[49] M. Pfender, N. Aslam, G. Waldherr, P. Neumann, and J. Wrachtrup. "Single-spin stochastic optical reconstruction microscopy". In: PNAS 111.41 (2014), pp. 14669-14674. DOI: 10 . 1073/pnas. 1404907111.

[50] S. Arroyo-Camejo et al. "Stimulated Emission Depletion Microscopy Resolves Individual Nitrogen Vacancy Centers in Diamond Nanocrystals". In: ACS Nano. 7.12 (2013), pp. 1091210919. DOI: $10.1021 / \mathrm{nn} 404421 \mathrm{~b}$. 
[51] E.H. Chen, O. Gaathon, M.E. Trusheim, and D. Englund. "Wide-Field Multispectral SuperResolution Imaging Using Spin-Dependent Fluorescence in Nanodiamonds". In: Nano lett. 15.5 (2013), pp. 2073-2077. DOI: 10.1021/n1400346k.

[52] G. Balasubsramanian et al. "Nanoscale imaging magnetometry with diamond spins under ambient conditions". In: Nature 455 (2008), pp. 648-651. DOI: 10.1038/nature07278.

[53] J.R. Maze et al. "Nanoscale magnetic sensing with an individual electronic spin in diamond". In: Nature 455 (2008), pp. 644-647. DOI: 10.1038/nature07279.

[54] L. Rondin et al. "Magnetometry with nitrogen-vacancy defects in diamond". In: Rep. Prog. Phys. 77.5 (2014). DOI: 10.1088/0034-4885/77/5/056503.

[55] F. Dolde et al. "Nanoscale detection of a single fundamental charge in ambient conditions using the $\mathrm{NV}^{-}$center in diamond". In: Phys. Rev. Lett. 112.097603 (9 2014). DoI: 10.1103/ PhysRevLett.112.097603.

[56] G. Kucsko et al. "Nanometre-scale thermometry in a living cell". In: Nature 500 (2013), pp. 54-58. DOI: doi:10.1038/nature12373.

[57] P. Neumann et al. "High-precision nanoscale temperature sensing using single defects in diamond". In: nano Lett. 13.6 (2013), pp. 2738-2742. DOI: 10.1021/nl401216y.

[58] J.F. Barry et al. "Optical magnetic detection of single-neuron action potentials using quantum defects in diamond". In: PNAS 113.49 (2016), pp. 14133-14138. DoI: 10.1073/pnas . 1601513113.

[59] T. A. Kennedy et al. "Single-Qubit Operations with the Nitrogen-Vacancy Center in Diamond". In: Phys.Stat. Sol.(b) 233.3 (2002), pp. 416-426. DOI: 10.1002/1521-3951(200210) 233:3<416: :AID-PSSB416>3.0.CO;2-R.

[60] C. Zu et al. "Experimental realization of universal geometric quantum gates with solid-state spins". In: Nature 514 (2014), pp. 72-75. DOI: 10.1038/nature13729.

[61] S. Arroyo-Camejo, A. Lazariev, S.W. Hell, and G. Balasubramanian. "Room temperature high-fidelity holonomic single-qubit gate on a solid state spin". In: Nat. Commun. 5.4870 (2014). DOI: $10.1038 /$ ncomms5870.

[62] L. Childress et al. "Coherent Dynamics of Coupled Electron and Nuclear Spin Qubits in Diamond". In: Science 314 (2006). DOI: 10.1126/science.1131871.

[63] L. Jiang et al. "Repetitive readout of single electronic spin via quantum logic with nuclear spin ancillae". In: Science 326 (5950 2009), pp. 267-272. DOI: 10.1126/science.1176496.

[64] P. Neumann et al. "Single-shot readout of single nuclear spin". In: Science 329 (5991 2010), pp. 542-544. DOI: $10.1126 /$ science. 1189075.

[65] P. Neumann et al. "Quantum register based on coupled electron spins in a room-temperature solid". In: Nat. Physics 6 (2010). DOI: 10.1038/NPHYS15360.

[66] D. Wildanger, J.R. Maze, and S.W. Hell. "Diffraction Unlimited All-Optical Recording of Electron Spin Resonances". In: Phys. Rev. Lett. 107.017601 (2011). DOI: $10.1103 /$ PhysRevLett.107.017601.

[67] N.Y. Yao et al. "Scalable architecture for a room temperature solid-state quantum information processor". In: Nat. Commun. 3.800 (2012). DOI: 10.1038/ncomms1788. 
[68] P. Spinicelli et al. "Engineered arrays of nitrogen-vacancy color centers in diamond based on implantation of CN- molecules through nanoapertures". In: New. J. Phys. 13.025014 (2011). DOI: $10.1088 / 1367-2630 / 13 / 2 / 025014$.

[69] I. Jakobi et al. "Efficient creation of dipolar coupled nitrogen-vacancy spin qubits in diamond". In: Journal of Physics: Conference Series 752.1 (2016). DOI: 10.1088/1742-6596/ 752/1/012001.

[70] W. Schnitzler et al. "Deterministic Ultracold Ion Source Targeting the Heisenberg Limit". In: Phys. Rev. Lett. 102.070501 (7 2009). DOI: 10.1103/PhysRevLett.102.070501.

[71] G. Jacob et al. "Transmission microscopy with nanometer resolution using a deterministic single ion source". In: Phys. Rev. Lett. 117.043001 (4 2016). DOI: 10.1103/PhysRevLett. 117.043001.

[72] P. Räcke, D. Spemann, J.W. Gerlach, B. Rauschenbach, and J. Meijer. "Detection of small bunches of ions using image charges". In: Sci. Rep. 8 (1 2018), p. 9781. DoI: 10.1038 / s41598-018-28167-6.

[73] S. Pezzagna, B. Naydenov, F. Jelezko, J. Wrachtrup, and J Meijer. "Creation efficiency of nitrogen-vacancy centres in diamond". In: N. J. Phys. 12.065017 (2010). DOI: 10.1088/ $1367-2630 / 12 / 6 / 065017$.

[74] F.F. de Oliveira et al. "Tailoring spin defects in diamond by lattice charging". In: Nat. Commun. 8.15409 (2017). DOI: 10.1038/ncomms15409.

[75] S. Onada et al. "Diffusion of vacancies created by high-energy heavy ion strike into diamond". In: Phys. Status Solidi A 214.1700160 (2017). DOI: 10.1002/pssa. 201700160.

[76] T. Lühmann et al. submitted for publication. 2018.

[77] S.-Y. Lee et al. "Readout and control of a single nuclear spin with a metastable electron spin ancilla". In: Nature Nanotech. 8 (2013). DOI: 10.1038/NNANO.2013.104.

[78] M. V. Berry. "Quantual Phase Factors Accompanying Adiabatic Changes". In: Proc. R. Soc. London, Ser. A 392.1802 (1984), pp. 45-57. DOI: 10.1098/rspa.1984.0023.

[79] G. Feng, G. Xu, and G. Long. "Experimental Realization of Nonadiabatic Holonomic Quantum Computation”. In: Phys. Rev. Lett. 110.190501 (2013). DOI: 10.1103/PhysRevLett. 110.190501.

[80] A.A. Abdumalikov Jr et al. "Experimental realization of non-Abelian non-adiabatic geometric gates". In: Nature 496 (2013). DoI: 10.1038/nature12010.

[81] Z.-T. Liang et al. "Proposal for implementing universal superadiabatic geometric quantum gates in nitrogen-vacancy centers". In: Phys. Rev. A. 93.040305(R) (2016). DOI: 10.1103/ PhysRevA.93.040305.

[82] C. Weitenberg et al. "Single-spin addressing in an atomic Mott insulator". In: Nature 471 (2011), pp. 319-324. DOI: 10.1038/nature09827.

[83] R. Blatt and D. Wineland. "Entangled states of trapped ions". In: Nature 453 (2008), pp. 1008-1015. DOI: 10.1038/nature07125.

[84] M.H. Anderson, J.R. Ensher, M.R. Matthews, C.E. Wiemann, and E.A. Cornell. "Observation of Bose-Einstein condensation inn a dilute atomic vapor". In: Science 14 (5221 1995), pp. 198-201. DOI: 10.1126/science.269.5221.198. 
[85] C. Robens et al. "High numerical aperture $(\mathrm{NA}=0.92)$ objective lens for imaging and addressing of cold atoms". In: Opt. Lett. 42.1043 (2017). DOI: 10.1364/OL.42.001043.

[86] A. Alberti et al. "Super-Resolution microscopy of single atoms in optical lattices". In: New J. Phys. 18.053010 (2016). DOI: 10.1088/1367-2630/18/5/053010.

[87] E. Abbe. "Beiträge zur Theorie des Mikroskops und der mikroskopischen Wahrnehmung". In: Arch. f. mikroskop. Anat. 9 (1873), pp. 413-468.

[88] S.W. Hell and J. Wichmann. "Breaking the diffraction resolution limit by stimulated emission: stimulated-emission-depletion fluorescence microscopy". In: Opt. Lett. 19.11 (1994), pp. 780-782. DOI: $10.1364 / 0 \mathrm{~L} .19 .000780$.

[89] B. Harke et al. "Resolution scaling in STED microscopy". In: Opt. Exp. 16.6 (2008). DOI: 10.1364/OE.16.004154.

[90] S.J. Sahl, S.W. Hell, and S. Jacobs. "Fluorescence nanoscopy in cell biology". In: Nat. Rev. Mol. Cell Biol. 18 (2017), pp. 685-701. DOI: 10.1038/nrm.2017.71.

[91] G.M. Akselrod, M.S. Akselrod, E.R. Benton, and N. Yasuda. "A novel Al2O3 fluorescent nuclear track detector for heavy charged particles and neutrons". In: Nucl. Instr. Meth. Phys. Res. B 247 (2 2006), pp. 295-306. DOI: 10.1016/j.nimb.2006.01.056.

[92] S. Onada et al. "New application of NV centers in CVD diamonds as a fluorescent nuclear track detector". In: Phys. Status Solidi A 212.11 (2015), pp. 2641-2644. DOI: 10.1002/ pssa. 201532219.

[93] N. Dwaikat and A.M. Al-Karmi. "Application of CR-39 Microfilm for Rapid Discrimination Between Alpha-Particle Sources”. In: NET 49 (2017), pp. 881-885. DOI: 10.1016/j . net. 2016.12.001.

[94] R. Félix-Bautista et al. "Evolution of etched nuclear track profiles of alpha particles in CR39 by atomic force microscopy". In: Rad. Meas 50 (2013), pp. 197-200. DoI: 10.1016/j . radmeas. 2013.01.002.

[95] S. Ota et al. "Tracking method for the measurement of projectile charge changing crosssection using CR-39 detector with high speed imaging microscope". In: Rad. Meas. 43 (2008), pp. 195-198. DOI: 10.1016/j.radmeas.2008.04.058.

[96] S. Greilich et al. "Fluorescent nuclear track detectors as a tool for ion-beam therapy research". In: Radiation Measurements 56.267-272 (2013). DOI: 10.11588/heidok.00017445.

[97] M. Niklas et al. "Spatial correlation between traversal and cellular response in ion radiotherapy - Towards single track spectroscopy". In: Rad. Meas. 56 (2013). DOI: 10.1016/j . radmeas. 2013.01.060.

[98] S. Onoda et al. "New application of NV centers in CVD diamonds as a fluorescent nuclear track detector". In: Phys. Status Solidi A 212.10 (2015), pp. 2641-2644. DOI: $10.1002 /$ pssa. 201532219.

[99] M. Gu. Advanced Optical Imaging. Springer Series in Optical Sciences, 1999.

[100] R. Hanburry Borwn and R. Q. Twiss. "Correlation between photos in two coherent beams of light". In: Nature 177 (1956), pp. 27-32. DoI: 10.1038/1781447a0.

[101] H. J. Kimble, M. Dagenais, and L. Mandel. "Photon Antibunching in Resonance Fluorescence". In: Phys. Rev. Lett. 39.691 (1977). DoI: 10.1103/PhysRevLett.39.691. 
[102] H. J. Carmichael and D. F. Walls. "Proposal for the measurement of the resonant Stark effect by photon correlation techniques". In: J. Phys. B: At. Mol. Phys. 9.L43 (1976). DoI: 10.1088/0022-3700/9/4/001.

[103] G. Vicidomini et al. "Sharper low-power STED nanoscopy by time gating". In: Nat. Methods 8 (2011), pp. 571-573. DOI: 10.1038/nMeth. 1624.

[104] M. Speidel, A. Jonáš, and E.-L. Florin. "Three-dimensional tracking of fluorescent nanoparticles with subnanometer precision by use of foff-focus imaging". In: Opt. Lett. 28.2 (2003). DOI: $10.1364 /$ OL. 28.000069.

[105] E. Toprak, H. Balci, B.H. Blehm, and P.R. Selvin. "Three-Dimensional Particle Tracking via Bifocal Imaging". In: Nano Lett. 7.7 (2007), pp. 2043-2045. DOI: 10.1021/n10709120.

[106] L. Weiss. "A reliable long-term tracking system for sub-diffraction far-field optical measurements on nitrogen vacancy centers in diamond". MA thesis. Georg-August-University Göttingen, 2017.

[107] M.J. Booth, M.A.A. Neil, R. Juškaitis, and T. Wilson. "Adaptive aberration correction in a confocal microscope". In: PNAS 99.9 (2002). DOI: 10.1073/pnas. 082544799.

[108] J. Hering, E.H. Waller, and G.v. Freymann. "Automated aberration correction of arbitrary laser modes in hgih numerical aperture systems". In: Opt. Express 24 (25 2016), pp. 2850028508. DOI: $10.1364 / 0 \mathrm{E} .24 .028500$.

[109] R. John, J. Lehnert, M. Mensing, S. Spemann D.and Pezzagna, and J. Meijer. "Bright optical centre in diamond with narrow, highly polarised and nearly phonon-free fluorescence at room temperature". In: New J. Phs. 19.053008 (2017). DOI: 10.1088/1367-2630/aa6d3f.

[110] P.J. Alt. "RESOLFT nanoscopy with water-soluble synthetic fluorophores". PhD thesis. Georg August University, Göttingen, 2018.

[111] J. Meijer et al. "Towards the implanting of ions and positioning of nanoparticles with $\mathrm{nm}$ spatial resolution". In: App. Phys. A 91 (2008), pp. 567-571. DOI: 10.1007/s00339-0084515-1.

[112] E. Rittweger. "Maximizing far-field optical microscopy resolution through selected fluorophore transitions". PhD thesis. Ruprecht-Karls-Universität Heidelberg, 2009.

[113] http://www.srim.org.

[114] K.T. Koga, J.A. Van Orman, and M.J. Walter. "Diffusive relaxation of carbon and nitrogen isotope heterogeneity in diamond: a new thermochronometer". In: Phys. Earth Planet. Inter. 139 (2003), pp. 35-43. DOI: 10.1016/S0031-9201(03)00141-9.

[115] T. Yamamoto et al. "Extending spin coherence times of diamond qubits by high-temperature annealing". In: Phys. Rev. B 88.075206 (7 2013). DOI: 10.1103/PhysRevB.88.075206.

[116] K. Iakoubovskii et al. "Annealing of vacancies and interstitials in diamond". In: Physica B 340-342 (2003), pp. 67-75. DOI: 10.1016/j.physb.2003.09.005.

[117] P.B. Stetson. "DAOPHOT: A computer program for crowded-field stellar photometry". In: Publ. Astron. Soc. Pac. 99.613 (1987), pp. 199-222. DOI: 10.1086/131977.

[118] Y. Aharonov and J. Anandan. "Phase Change during a cyclic quantum evolution". In: Phys. Rev. Lett. 58.1593-6 (1987). DOI: 10.1103/PhysRevLett.58.1593.

[119] A.K. Pati. "Gauge-invariant reference section and geometric phase". In: J. Phys. A:Math. Gen. 28 (1995), pp. 2087-2094. DOI: 10.1088/0305-4470/28/7/027. 
[120] G. Falci, R. Fazio, G.M. Palma, J. Siewert, and V. Vedral. "Detection of geometeric phases in superconducting nanocircuits". In: Nature 407 (2000), pp. 355-358. DoI: $10.1038 /$ 35030052 .

[121] J. A. Jones, V. Vedral, A. Ekert, and G. Castagnoli. "Geometric quantum computation using nuclear magnetic resonance". In: Nature 405 (2000), pp. 869-871. DOI: 10.1038/35002528.

[122] X.-B. Wang and M. Keiji. "Nonadiabatic Conditional Geometric Phase Shift with NMR". In: Phys. Rev. Lett 87.9 (2001). DOI: 10.1103/PhysRevLett.87.097901.

[123] P. Zanardi and M. Rasetti. "Holonomic quantum computation". In: Phys. Lett. A 264 (1999). DOI: $10.1016 /$ S0375-9601(99) 00803-8.

[124] F. Wilczek and A. Zee. "Appearance of Gauge Structure in Simple Dynamical Systems". In: Phys. Rev. Lett. 52.24 (1984). DOI: 10.1103/PhysRevLett.52.2111.

[125] K. Toyoda, K. Uchida, A. Noguchi, S. Haze, and S. Urabe. "Realization of holonomic singlequbit operations". In: Phys. Rev. A 87.052307 (2013). DOI: 10.1103/PhysRevA.87.052307.

[126] L.-M. Duan, J.I. Cirac, and P. Zoller. "Geometric manipulation of trapped ions for quantum computation". In: Science 292.5522 (2001). DOI: 10.1126/science.1058835.

[127] E. Sjöqvist et al. "Non-adiabatic holonomic quantum computation". In: New J. Phys. 14.10 (2012), p. 103035. DOI: 10.1088/1367-2630/14/10/103035.

[128] Y. Sekiguchi, N. Niikura, R. Kuroiwa, H. Kano, and H. Kosaka. "Optical holonomic single quantum gates with a geometric spin under a zero field". In: Nat. Photonics 11 (2017), pp. 309-314. DOI: 10.1038/nphoton.2017.40.

[129] B.B. Zhou et al. "Holonomic quantum control by coherent optical excitation in diamond". In: Phys. Rev. Lett. 119.140503 (2017). DoI: 10.1103/PhysRevLett.119.140503.

[130] M. Fleischhauer and A.S. Manka. "Propagation of laser pulses and coherent population transfer in dissipative three-level systems: an adiabatic dressed-state picture". In: Phys. Rev. A 54.794 (1996). DOI: 10.1103/PhysRevA.54.794.

[131] Y. Aharonov and D. Bohm. "Significance of electromagnetic potentials in the quantum theory". In: Physical Review 115.3 (1959), pp. 485-491. DOI: 10.1103/PhysRev.115.485.

[132] S. Filipp. "Noise fluctuations and the Berry phase: Towards an experimental test". In: Eur. Phys. J. Special Topics 160 (1 2008), pp. 165-174. DOI: 10.1140/epjst/e2008-00720-1.

[133] S. Filipp et al. "Experimental demonstration of the stability of Berry's phase for a spin1/2 particle". In: Phys. Rev. Lett. 102.030404 (3 2009). DOI: 10.1103/PhysRevLett. 102. 030404 .

[134] G. De Chiara and G.M. Palma. "Berry phase for a spin 1/2 particle in a classical field". In: Phys. Rev. Lett. 91.9 (9 2003).

[135] P.J. Leek et al. "Observation of Berry's phase in a solid-state qubit". In: Science 318 (5858 2007), pp. 1889-1892. DOI: 10.1126/science.1149858.

[136] S. Berger et al. "Exploring the effect of noise on the Berry phase". In: Phys. Rev. A 87.0603030(R) (6 2013). DOI: 10.1103/PhysRevA.87.060303.

[137] M. Johansson et al. "Robustness of nonadiabatic holonomic gates". In: Phys. Rev. A 86.062322 (6 2012). DOI: 10.1103/PhysRevA.86.062322.

[138] D.F.V. James, P.G. Kwiat, W.J. Munro, and A.G. White. "Measurement of qubits". In: Phys. Rev. A 64.052312 (5 2001). DOI: 10.1103/PhysRevA.64.052312. 
[139] J.L. O'Brien et al. "Quantum Process Tomography of a Controlled-Not Gate". In: Phys. Rev. Lett. 93.8 (2004). DOI: 10.1103/PhysRevLett93.080502.

[140] M. Howard et al. "Quantum process tomography and Linblad estimation of a solid-state spin”. In: New J. Phys. 8.33 (2006). DoI: https://doi .org/10.1088/1367-2630/8/3/033.

[141] S. Arroyo-Camejo, A. Lazariev, S.W. Hell, and G. Balasubramanian. "Supplementary Information Room temperature high-fidelity holonomic single-qubit gate on a solid state spin". In: Nat. Commun. 5.4870 (2014). DOI: 10.1038/ncomms5870.

[142] S. Arroyo-Camejo. "Quantum information, quantum sensing, and super-resolution microscopy with nitrogen-vacancy centers in diamond". PhD thesis. Ruprecht-Karls-Universität Heidelberg, 2015.

[143] E. Knill et al. "Randomized benchmarking of quantum gates". In: Phys. Rev. A 77.012307 (2008). DOI: 10.1103/PhysRevA .77.012307.

[144] J.P. Geabler et al. "Randomized Benchmarking of Multiqubit Gates". In: Phys. Rev. Lett. 108.260503 (2012). DOI: 10.1103/PhysRevLett.108.260503.

[145] C.A. Ryan, M. Laforest, and R. Laflamme. "Randomized benchmarking of single- and multi-qubit control in liquid-state NMR quantum information processing". In: New J. Phys. 11.013034 (2009). DOI: 10.1088/1367-2630/11/1/013034.

[146] J. Kelly et al. "Optimal Quantum Control Using Randomized Benchmarking". In: Phys. Rev. Lett. 112 (24 2014), pp. 240504-5. DOI: 10.1103/PhysRevLett.112.240504.

[147] J.M: Chow et al. "Randomized Benchmarking and Process Tomography for Gate Errors in a Solid-State Qubit". In: Phys. Rev. Lett. 102.090502 (2009). DOI: 10.1103/PhysRevLett. 102.090502 .

[148] K.R. Brown et al. "Single-qubit-gate error below $10^{-4}$ in a trapped ion". In: Phys. Rev. A 84.030303(R) (3 2011). DOI: 10.1103/PhysRevA.84.030303.

[149] D. Wildanger. "Progress in simulated emission depletion (STED) microscopy including the optical detection of magnetic resonances of single spins beyond the diffraction limit". $\mathrm{PhD}$ thesis. Ruprecht-Karls-Universität Heidelberg, 201.

[150] M.V. Berry. "Transitionless quantum driving". In: J. Phys. A: Math 42.365303 (2009). DoI: 10.1088/1751-8113/42/36/365303.

[151] D. Sels and A. Polkovnikov. "Minimizing irreversible losses in quantum systems by local counterdiabatic driving". In: PNAS 114.20 (2017). DOI: 10.1073/pnas.1619826114.

[152] M. Demirplak and S.A. Rice. "Adiabatic population transfer with control fields". In: $J$. Phys. Chem. A 107.46 (2003), pp. 8837-9945. DOI: 10.1021/jp030708a.

[153] R. Lim and M.V. Berry. "Superadiabatic tracking of quantum evolution". In: J. Phys. A: Math. Gen. 24.14 (1991). DOI: 10.1088/0305-4470/24/14/014.

[154] F.Q. Dou, J. Liu, and L.-B. Fu. "High-fidelity superadiabatic population transfer of a twolevel system with a linearly chirped Gaussian pulse". In: EPL 116.6 (2016). DOI: 10.1209/ 0295-5075/116/60014.

[155] M.G. Bason et al. "High-fidelity quantum driving". In: Nat. Phys. 8 (2012), pp. 147-152. DOI: $10.1038 /$ nphys 2170 .

[156] N. Malossi et al. "Quantum driving protocols for a two-level system: From generalized Landau-Zener sweeps to transitionless control". In: Phys. Rev. A. 87.012116 (1 2013). 
[157] B.B. Zhou et al. "Accelerated quantum control using superadiabatic dynamics in a solidstate lambda system". In: Nat. Phys. 13 (2017), pp. 330-334. DOI: 10.1038/nphys3967.

[158] Y.-X. Du et al. "Experimental realization of stimulated Raman shortcut-to-adiabatic passage with cold atoms". In: Nat. Commun. 7.12479 (2016). DOI: 10.1038/ncomms12479.

[159] C. Zener. "Non-adiabatic crossing of energy levels". In: Proc. Roy. Soc. A 137 (833 1932). DOI: $10.1098 /$ rspa.1932.0165.

[160] S. Zhu and Z.D. Wang. "Implementation of Universal Quantum Gates Based on Nonadiabatic Geometric Phases". In: Phys. Rev. Lett. 89.9 (2002). DoI: 10.1103/PhysRevLett.89. 097902.

[161] S.-L. Zhu and Z.D. Wang. "Universal quantum gates based on a pair of orthogonal cyclic states: Application to NMR systems". In: Phys. Rev. A 67.022319 (2003). DoI: $10.1103 /$ PhysRevA.67.022319.

[162] S.L. Zhu, Z.D. Wang, and P. Zanardi. "Geometric Quantum Computation and Multiqubit Entanglement with Superconducting Qubits inside a Cavity". In: Phys. Rev. Lett. 94.100502 (2005). DOI: 10.1103/PhysRevLett.94.100502.

[163] H. WU et al. "Geometric phase gates with adiabatic control in electron spin resonance". In: Phys. Rev. A 87.032326 (2013). DOI: 10.1103/PhysRevA.87.032326.

[164] A.Yu. Kitaev. "Quantum computations: algorithms and error correction". In: Russ. Math. Surv. 52.1191 (1997).

[165] A.G. Fowler. "Surface codes: Towards practical large-scale quantum computation". In: Phys. Rev. A 86.032324 (2012). DOI: 10.1103/PhysRevA.86.032324.

[166] Y. Romach et al. "Spectroscopy of Surface-Induced Noise Using Shallow Spins in Diamond". In: Phys. Rev. Lett. 114.017601 (2015). DOI: 10.1103/PhysRevLett.114.017601.

[167] R.M. and Lutchyn, C.P. Nave, and S. Das Sarma. "How to enhance dephasing time in superconducting qubits". In: Phys. Rev. B 77.174509 (17 2008). DOI: $10.1103 /$ PhysRevB. 77.174509 .

[168] J. Casanova, Z.-Y. Wang, J.F. Haase, and M.B. Plenio. "Robust dynamical decoupling sequences for individual-nuclear-spin addressing". In: Phys. Rev. A 92.042304 (4 2015). DOI: $10.1103 /$ PhysRevA.92.042304.

[169] Y. Romach et al. "Quantum noise spectroscopy of non-monotonous spectra". arXiv:1803.07390. 2018.

[170] S. Pezzagna et al. "Creation of colour centres in diamond by collimated ion-implantation through nano-channels in mica". In: Phys. Status Solidi A 208.9 (2011). DOI: 10.1002 / pssa. 201100455.

[171] X. Rong et al. "Supplementary Information: Experimental fault-tolerant universal quantum gates with sold-state spins under ambient conditions". In: Nat. Commun. 6.8748 (2015). DOI: 10.1038/ncomms 9748 .

[172] E. Sjöqvist, V.A. Mousolou, and C.M. Canali. "Conceptual aspects of geometric quantum computation". In: Quantum Inf Process 15 (10 2016), pp. 3995-4011. DOI: 10.1007/s11128016-1381-1.

[173] N.M. Linke et al. "Experimental comparison of two quantum computing architectures". In: PNAS 114.13 (2017), pp. 3305-3310. DOI: 10.1073/pnas.1618020114. 
[174] E. Ferraro, M. Fanciulli, and M. De Michielis. "Gate fidelity comparison in semiconducting spin qubit implementations affected by control noises". arXiv:1806.07597. 2018.

[175] Z.-T. Liang et al. "Supplementary Material for proposal for implementing universal superadiabatic geometric quantum gates in nitrogen-vacancy centers". In: Phys. Rev. A. 93.040305(R) (2016). DOI: 10.1103/PhysRevA.93.040305.

[176] V.M. Acosta et al. "Temperature Dependence of the Nitrogen-Vacancy Magnetic Resonance in Diamond". In: Phys. Rev. Lett. 104 (7 2010), p. 070801. DoI: 10.1103/PhysRevLett. 104.070801.

[177] J. Koch et al. "Charge-insensitive qubit design derived from the Cooper pair box". In: Phys. Rev. A 76 (4 2007), p. 042319. DOI: 10.1103/PhysRevA.76.042319.

[178] J. A. Schreier et al. "Suppressing charge noise decoherence in superconducting charge qubits". In: Phys. Rev. B 77 (18 2008), p. 180502. DOI: 10.1103/PhysRevB.77.180502.

[179] D.H. Slichter et al. "Measurement-Induced Qubit State Mixing in Circuit QED from UpConverted Dephasing Noise”. In: Phys. Rev. Lett. 109 (15 2012), p. 153601. DoI: 10.1103/ PhysRevLett.109.153601.

[180] F. Motzoi, J. M. Gambetta, P. Rebentrost, and F. K. Wilhelm. "Simple Pulses for Elimination of Leakage in Weakly Nonlinear Qubits". In: Phys. Rev. Lett. 103 (11 2009), p. 110501. DOI: 10.1103/PhysRevLett.103.110501.

[181] A.A. Abdumalikov Jr et al. "Supplementary Information: Experimental realization of nonAbelian non-adiabatic geometric gates". In: Nature 496 (2013). DOI: 10.1038/nature12010.

[182] B.K. Ofori-Okai et al. "Spin properties of very shallow nitrogen vacancy defects in diamond". In: Phys. Rev. B 86.081406(R) (2012). DOI: 10.1103/PhysRevB.86.081406.

[183] A. Jarmola, V.M. Acosta, K. Jensen, S. Chemerisov, and D. Budker. "Temperature- and Magnetic-Field-Dependent Longitudinal Spin Relaxation in Nitrogen-Vacancy Ensembles in Diamond". In: Phys. Rev. Lett. 108.197601 (19 2012). DOI: 10.1103/PhysRevLett.108. 197601.

[184] M.O. Scully and M.S. Zubairy. Quantum Optics. Cambridge: Cambridge Univ. Press, 1997.

[185] J.R. Maze et al. "Free induction decay of single spins in diamond". In: New J. Phys. 14.103041 (2012), p. 17. DOI: 10.1088/1367-2630/14/10/103041.

[186] G. de Lange, T. van der Sar, M. Blak, Z.-H. Wang, and V. Dobrovitski. "Controlling the quantum dynamics of a mesoscopic spin bath in diamond". In: Sci. Rep. 2.382 (2012). DoI: 10.1038/srep00382.

[187] E. L. Hahn. "Spin Echoes". In: Phys. Rev. 80.4 (1950). DOI: 10.1103/PhysRev. 80.580.

[188] L.M. Pham et al. "Enhanced solid-state multispin metrology using dynamical decoupling". In: Phys. Rev. B 86.045214 (4 2012). DOI: 10.1103/PhysRevB.86.045214.

[189] N. Bar-Gill et al. "Suppression of spin-bath dynamics for improved coherence of multi-spinqubit systems". In: Nat. Commun. 3.858 (2012). DOI: 10.1038/ncomms 1856.

[190] B.E. Kane. "A silicon-based nuclear spin quantum computer". In: Nature 393 (1998). DOI: $10.1038 / 30156$.

[191] M. Steiner, P. Neumann, J. Beck, F. Jelezko, and J. Wrachtrup. "Universal enhancement of the optical readout fidelity of single electron spins at nitrogen-vacancy centers in diamond". In: Phys. Rev. B 81.035205 (2010). DOI: 10.1103/PhysRevB.81.035205. 
[192] L.P. McGuiness et al. "Quantum measurement and orientation tracking of fluorescent nanodiamonds inside living cells". In: Nat. Nanotechnol. 6 (2011), pp. 358-363. DOI: 10.1038/ nnano.2011.64.

[193] A. Dréau et al. "Avoiding power broadening in optically detected magnetic resonance of single NV defects for enhanced dc magnetic field sensitivity". In: Phys. Rev. B. 84.195204 (2011). DOI: 10.1103/PhysRevB.84.195204. 


\section{A. Data acquisition}

Some of the presented data required significant averaging including frequent retuning of the control parameter. Here, the most important features of the hard and software, needed for the automation of the experimental apparatus, are given. This knowledge is not necessarily required for the understanding of the findings within this thesis but supports attempts which aim to reproduce the data.

\section{A.1. Electronic control circuit}

The experimental control software distinguishes two different operational modes: experiment mode and imaging mode. The main difference between the modes is the way how the data acquisition of the multichannel scalar (MCS) is triggered. In imaging mode, used for performing confocal and super-resolution microscopy, as well as for the refocusing of the defect center interleaved with the experiments, the trigger of the acquisition is provided by the STED laser directly. Data acquisition starts with the first trigger arriving after the MCS is armed (inset Fig. A.1). However, during coherent spin manipulating experiments (experiment mode) the data acquisition needs to be synchronized with the experimental sequence in order to enable time-gated detection. Therefore, in this mode the trigger needs to be provided by the AWG, which itself is synchronized with the STED laser. Whenever readout of the spin state is required the AWG starts the MCS after detection of the next STED laser trigger (inset Fig. A.1). In the described configuration the STED laser is internally triggered and acts as a master for all operational modes. Since the STED laser is equipped with an unstoppable power supply it is protected against self damaging caused by loss of an external trigger signal and power failure.

The desired behaviour is obtained by an electronic circuit (Fig. A.1) which is controlled by the experiment computer via a National Instruments data acquisition (NIDAQ) card (PCIe-6353, National Instruments). First, a card duplicates the $30 \mathrm{MHz}$ STED laser reference. A pico-second delay unit provides the opportunity to precisely tune the optical delay between excitation (EXC) and STED pulses. Two additional copies of the reference are utilized for the switching between experiment and imaging mode. One copy is either transmitted or blocked by a logic AND-card whose second entrance port is controlled via the NIDAQ-card. The second copy is provided to the AWG for synchronization in experiment mode. The synchronized marker output of the AWG is directed to the MCS via a second AND-logic, as well controlled by the NIDAQ-card. The combination of NIDAQ-card and AND-logic cards allows for deterministic switching between the two trigger channels by setting one or the other NIDAQ channels to high. An additional ORlogic card sums up the signals provided by both AND-gates and directs the new signal to the start-channel of the multiple-event time digitizer. Note, for continuous wave (CW) excitation the synchronization of the AWG to the STED laser is not required. However, in order to keep the experiment control software as compact and robust as possible it does not distinguish between $\mathrm{CW}$ and pulsed laser mode. 


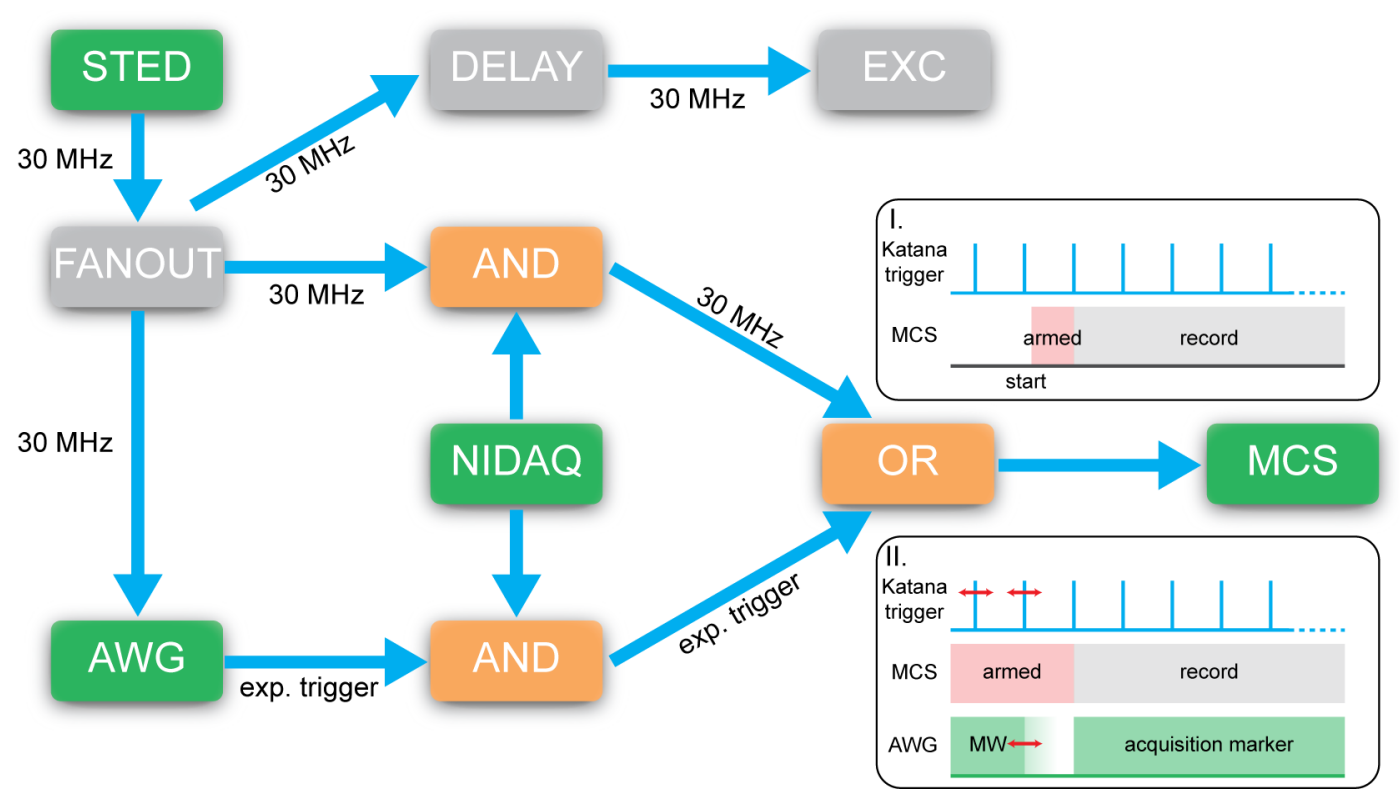

Figure A.1.: Simplified sketch of the electronic control circuit. The fanout card (grey) provides multiple copies of the $30 \mathrm{MHz}$ reference signal. Orange boxes represent classical logic gates. Devices shown as green box exhibit a direct connection to the control computer. Depending on the output of the NIDAQ-card the multiple-event time digitizer card is either trigger by the laser directly or indirectly by the arbitrary waveform generator.

\section{A.2. Superscript data acquisition}

In order to perform automatized long-term measurements featuring periodic retuning of the physical control parameter an experimental routine defined as SuperScript was implemented. In intervals predefined by the user the routine measures the ESR spectra and performs a Rabi measurement in order to retune the systems resonance frequency $\omega_{0}$ and Rabi frequency $\Omega$. Based on the refreshed values $\omega_{0}$ and $t_{\pi}=\pi / \Omega$ the main experiment is updated in order to correct for slow drifts of the system and measure close to the optimal working point (Sec. A.3). In case the main experiment is performed on both NV center transitions two ESR and Rabi measurements are taken. Figure A.2.a illustrates the SuperScript data acquisition in a flow chart. Obviously, the routine continuous with the next main experiment if it is neither time for a ESR or Rabi measurement.

To avoid an unnecessary time overhead at the beginning of each experiment, it is checked if the experiment changed and a new waveform needs to be created (Fig. A.2.b). Depending on the main experiment up to several ten minutes of waveform creation and upload are saved. The experiment timer controls if the anticipated number of averages has been recorded or a repositioning of the NV center within the focal volume is needed. In case of a refocusing a xy and xz-scan of the NV center is performed and its center position determined by two-dimensional Gaussian fits. After repositioning the NV center the measurement is continued. Refocusing is adjusted depending on the sample and typically takes two minutes. In future, an active sample stabilization will reduce the time overhead as investigated in [106].

\section{A.3. Resonance frequency tracking}

In this Ch. 12, the robustness of different quantum gate modalities with respect to controlled experimental control parameter imperfections is analysed. Obviously, such kind of measurements 
a

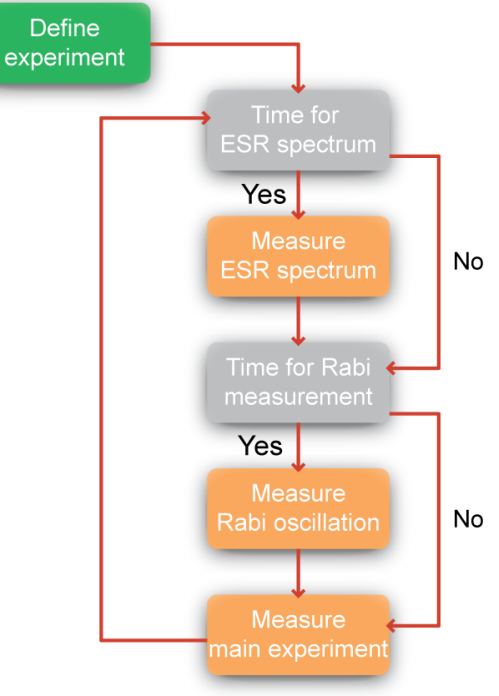

b

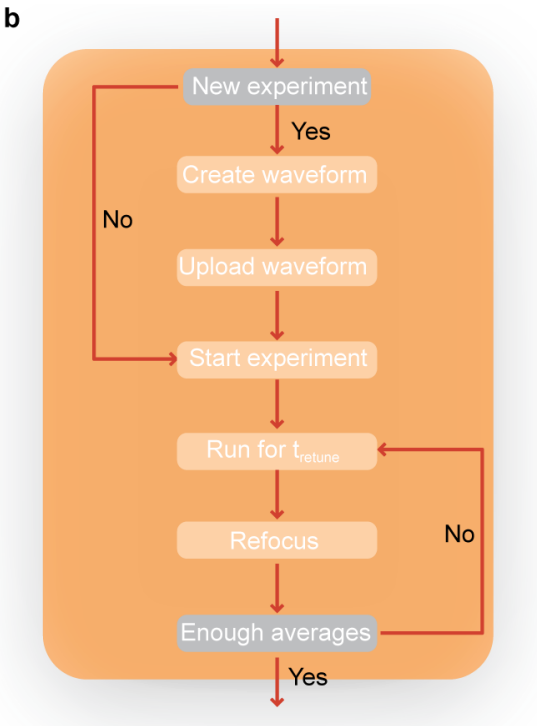

Figure A.2.: Flowchart of the superscript data acquisition. (a) (b) At the beginning of each experiment it is checked if the required experiment is identical to the previous on. If so the experiment is started immediately. Otherwise the desired waveforms are created and uploaded to the AWG. Subsequently the experiment is started and run for a predefined time

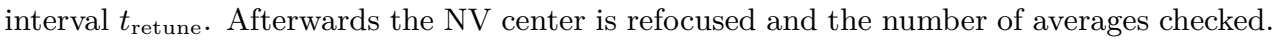
If enough statistics have been obtained the software moves on to the next experiment. Otherwise it is averaged for another $t_{\text {retune. }}$

require close tracking of the ESR resonance and Rabi frequency to avoid distortions of the result due to drifts of the system. Therefore, the actual quantum process tomography measurements are taken interleaved with ESR spectra and Rabi measurements used for retuning of the control parameter (Sec. A.2). Retuning intervals are typically set to $3-4 \mathrm{~h}$ and require (depending on the number of driving fields $) \approx 25-50 \mathrm{~min}$, significantly prolonging the acquisition time.

In order to extract the resonance frequencies $\left(\omega_{0-}, \omega_{0+}\right)$ and Rabi frequencies $\left(\Omega_{0,-}, \Omega_{0,+}\right)$ a Lorentzian function is fitted to the ESR resonances and a cosine to the Rabi measurements, respectively. Figure A.3.a-b shows an exemplary ESR resonance and Rabi frequency retuning trace over the acquisition time of one QPT detuning analysis curve like presented in Sec. 12.5. While the measured Rabi frequency remains stable within the experimental uncertainties, the ESR resonance shows temporal changes correlated with the temperature of the laboratory (Fig. A.3.c). The small temporal delay between drifts of the ESR resonance and laboratory temperature is dedicated to the fact that the temperature was measured outside the acoustic isolation box containing the objective lens and sample. The fitting accuracy is exceptionally high $\left(\Delta \omega_{0-} \approx \pm 25 \mathrm{kHz}\right)$ while the temperature induced drift of the resonance ranges up to $\sim 100 \mathrm{kHzh}^{-1}$. Measuring close to the optimal working point is guaranteed by rejecting measurement intervals suffering from ESR resonance drifts larger than $\pm 150 \mathrm{kHz}$. 
a
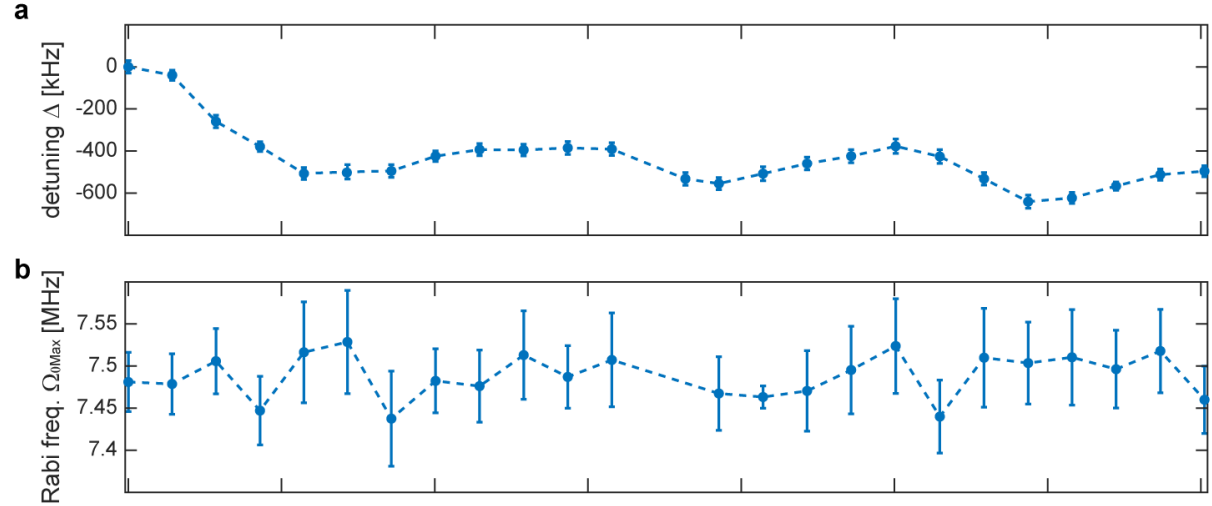

C

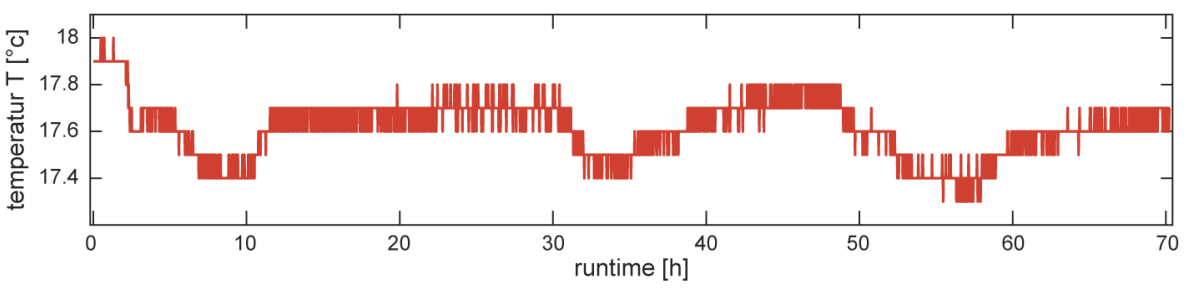

Figure A.3.: Tracking of the experiment control parameter. (a) Measured drift $\delta$ of ESR resonance with respect to the starting value over the runtime. (b) Measured Rabi frequency value $\Omega$ at maximal driving field strength over the runtime. (c) Laboratory temperature as measured outside of the acoustic isolation box of the sample. Lines are guide to the eye. While the Rabi frequency is stable the ESR resonance is correlated with the ambient temperature. 


\section{B. Sample characterization}

The diamond sample utilized in the quantum information measurements (Ch. 11 and Ch. 12) was optimized to contain as few imperfections interacting with the NV center electron spin as possible. Based on a diamond substrate with natural abundance $(\sim 1.1 \%)$ of ${ }^{13} \mathrm{C}$ an isotopically pure $50 \mu \mathrm{m}$ thick layer with $99.999 \%$ abundance of ${ }^{12} \mathrm{C}$ was grown (Element6). The sample was used as grown in order to avoid strain induced by surface polishing, reducing the coherence properties of the $\mathrm{NV}$ centers. NV centers were generated by microbeam implantation of high energetic $(10 \mathrm{MeV})$ ${ }^{14} \mathrm{~N}$ ions and subsequent annealing at $1000{ }^{\circ} \mathrm{C}$ for $2 \mathrm{~h}$ under vacuum. An average implantation dose of approx. 2 ions/spot in combination with the finite conversion efficiency results in single NV center. The high energetic implantation positioning the nitrogen ions $3.7 \mathrm{\mu m}$ underneath the diamond surface omits the interaction of surface related spin noise $[182,166]$ with the targeted NV center electron spin. Full description of the resulting NV center coherence properties at roomtemperature and a magnetic field of $|B| \approx 406 \mathrm{G}$ aligned parallel to the NV center axis is provided in the following.

\section{B.1. NV center coherence times}

The longitudinal relaxation time $T_{1}$ describes the population loss due to stochastic processes as, e.g., phonon interactions which are irreversible [183]. Experimentally it is measured by initializing the system into the $| \pm 1\rangle$ state and readout of the population after time $\tau$. An exponential fit of the form $M(\tau)=C\left(1-2 \cdot \exp \left(-\tau / T_{1}\right)\right)$, where $C$ is the signals contrast, reveals $T_{1}=13.7 \pm 2.2 \mathrm{~ms}$ (Fig. B.1.a).

$T_{1 \rho}$ is the longitudinal relaxation time in the rotating frame and is measured by a spin-lock experiment. In this sequence, after the application of a $\pi / 2$-pulse a, $90^{\circ}$ phase shifted, continuous driving field of duration $\tau$ locks the spin in its state. A second $\pi / 2$-pulse at the end of the sequence projects the relaxation into a measurable population difference. By fitting the function $M(\tau)=C \exp \left(-\tau / T_{1 \rho}\right)$ the relaxation time is determined to be $T_{1 \rho}=5.05 \pm 1.56 \mathrm{~ms}$ approaching the ultimately limiting $T_{1}$ time (Fig. B.1.b).

A Ramsey experiment extracts the spin dephasing/free induction decay (FID) time $T_{2}^{*}$ caused by field inhomogeneities [184]. After initialization a $\pi / 2$-pulse converts the $|0\rangle$ into the superposition state $\frac{1}{\sqrt{2}}(|+\rangle-i|-\rangle)$ and the system evolves freely for time $\tau$. An additional $\pi / 2$-pulse projects the obtained state back into the population basis $m_{s}=0$ and $m_{s}=-1$ [185]. Fig. B.1.c shows the measured FID decay signal. The fast oscillation originates from the detuning $\delta$ of the second $\pi / 2$-pulse while the exponential decay of the envelope is the $T_{2}^{*}$-constant. Fitting the experimental data by $M(\tau)=C \exp \left(-t / T_{2}^{*}\right) \cos (2 \pi \delta t)$ reveals a spin dephasing time of $T_{2}^{*}=4.25 \pm 0.27 \mu \mathrm{s}$. The absence of a beating within the decay curve is an indication of a properly polarized ${ }^{14} \mathrm{~N}$ nuclear spin [186] (Sec. B.2).

Finally, the spin-spin realization time $T_{2}$ caused by random magnetic field fluctuations, commonly introduced by surrounding nuclear spins, is measured by means of a Hahn-Echo experiment $[187,62]$. Hahn-echo experiments extent the Ramsey experiment by an additional $\pi$-pulse applied 

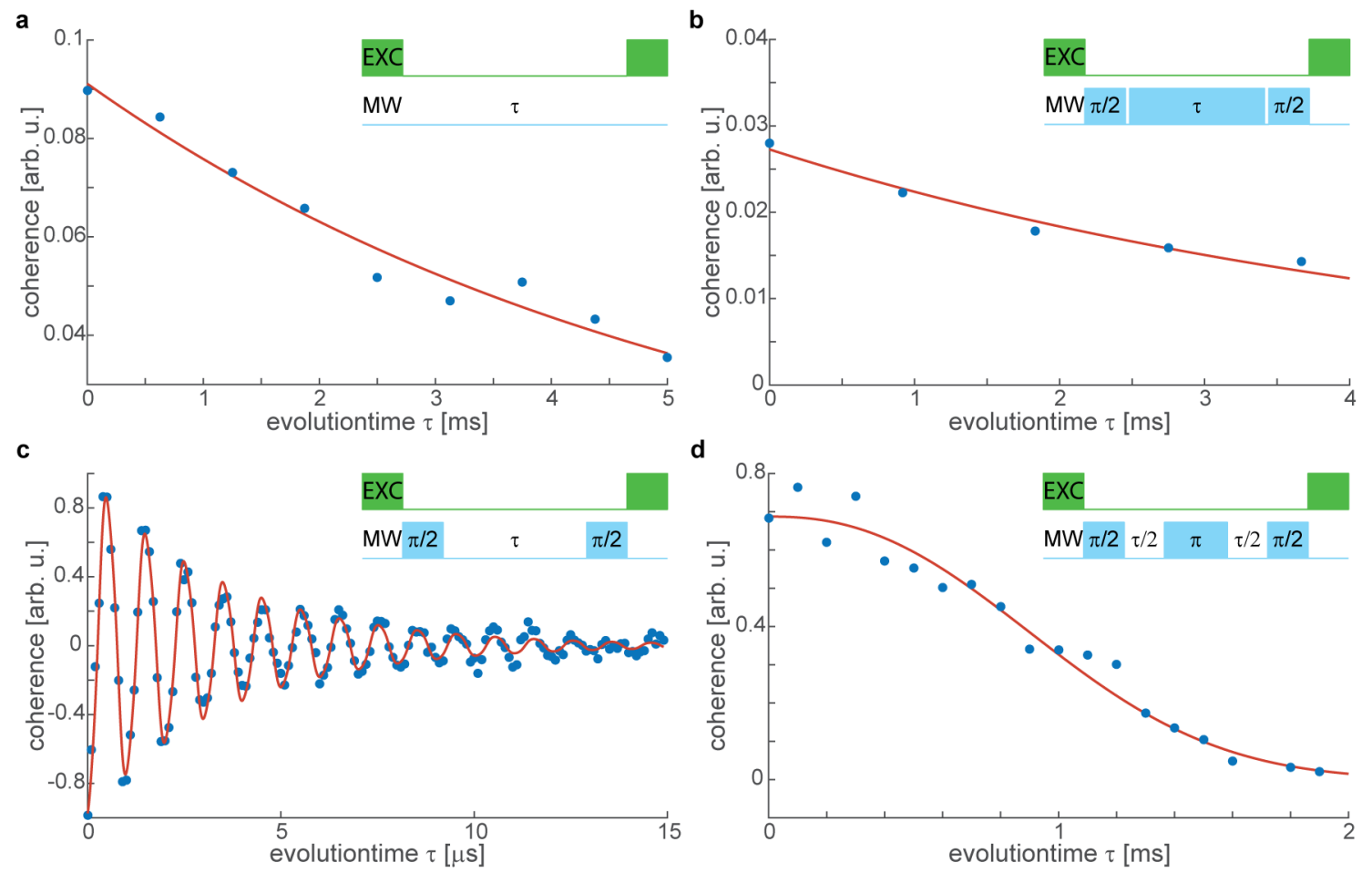

Figure B.1.: Measured diamond coherence properties. (a) Spin-lattice relaxation, (b) spin-lock, (c) Ramsey and (d) Hahn-Echo measurements (blue) and the respective fitted decay function (red, explicitly written in the text). Insets illustrate the measurement sequence, where green rectangular indicate laser pulses and blue rectangular indicate microwave fields.

at half the free evolution time $(t=\tau / 2)$, leading to a refocusing of the spin at time $\tau$. After fitting the functional $M(\tau)=\exp \left(-\tau / T_{2}\right)^{p}$, the spin-spin relaxation time is determined to be $T_{2}=1.13 \pm 0.09 \mathrm{~ms}$ and the parameter $p=2.36 \pm 0.66$ is between 1 and 2.5 as required from theory $[188,189]$.

In conclusion, the long coherence times make the sample highly suitable for quantum information applications, even at room-temperature. Particularly, the absence of spin impurities allows for the analysis of the quantum gate performance limited by controlled experimental parameter imperfections rather than uncontrolled sample related environmental induced noise.

\section{B.2. Nuclear spin polarization}

Due to hyperfine coupling of the NV center electron spin with the nuclear spin of the host ${ }^{14} \mathrm{~N}$ nitrogen atom $(I=1)$ the electron spin resonance (ESR) spectrum in general shows three resonances for each of the transitions $|0\rangle \leftrightarrow|-\rangle$ and $|0\rangle \leftrightarrow|+\rangle$, such that in general the experimental system is described by a six level system. However, after polarization of the nuclear spin, due to the long nuclear spin coherence time of several seconds [190, 37], measurements can be performed in a close to perfect two-level system. It was shown that dynamic polarization of the nitrogen nuclear spin is achieved by tuning to the excited state level anti-crossing (ESLAC) by application of a magnetic field [39]. In order to polarize the nuclear spin into $m_{I}=1$ a magnetic field of about $|B| \approx 400 \mathrm{G}$ needs to be aligned along the NV center $\hat{z}$ axis. Under green illumination the electron spin is continuously pumped into $m_{s}=0$, while flip-flop process transfer the nuclear spin into $m_{s}=+1$ [191]. A more detailed description of the physical process is presented in [40,39, 191], while here 

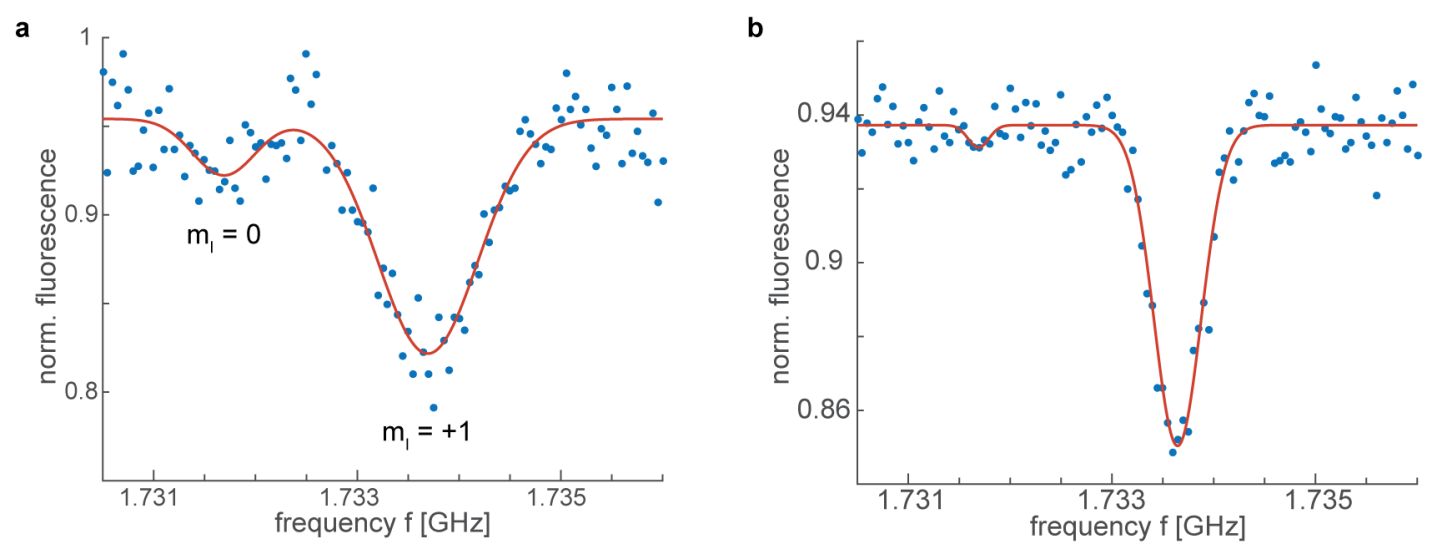

Figure B.2.: Nuclear spin polarization. ESR spectra of an NV center with a magnetic field of $|B| \approx$ $406 \mathrm{G}$ aligned (a) off-axis and (b) on-axis with respect to the NV center and a calculated nuclear polarization of $74 \%$ (94\%), respectively.

only its implication is of interest.

As reference for the coarse alignment of the magnetic field with respect to the NV center $\hat{z}$ axis the ESR spectra for both transitions, $|0\rangle \leftrightarrow|-\rangle$ and $|0\rangle \leftrightarrow|+\rangle$, are recorded. Out of the peak position (fitted by a Gaussian function) of the resonance features $\nu_{+}$and $\nu_{-}$the magnetic field strength $\left|B_{\text {ext }}\right|[\mathrm{G}]$ and the field angle $\theta_{\text {ext }}[\mathrm{rad}]$ can be estimated according to $[52,192]$

$$
\begin{aligned}
\left|B_{\mathrm{ext}}\right| & =\sqrt{\frac{1}{3 \gamma^{2}}\left(\nu_{+}^{2}+\nu_{-}^{2}-\nu_{+} \nu_{-}-D^{2}\right)^{2}} \\
\theta_{\mathrm{ext}} & =\frac{1}{2} \arccos \left(\frac{7 D^{3}+2\left(\nu_{+}+\nu_{-}\right)^{2} \cdot\left(2\left(\nu_{+}^{2}+\nu_{-}^{2}\right)-5 \nu_{+} \nu_{-}\right)-3 D\left(\nu_{+}^{2}+\nu_{-}^{2}-\nu_{+} \nu_{-}\right)}{9 D\left(\nu_{+}^{2}+\nu_{-}^{2}-\nu_{+} \nu_{-}-D^{2}\right)^{2}}\right)
\end{aligned}
$$

where $\gamma$ is the electron gyro magnetic ration and $D \approx 2.87 \mathrm{GHz}$ the zero field splitting. Iterative optimization of the magnetic field aligns the magnetic field with several degree precision. However, the estimated field angle is only an approximation, since the exact value of $\Delta_{0}$ is a property of the diamond sample and difficult to determine due to the presence of the earth magnetic field and strain within the diamond.

Hence, fine tuning is performed by iteratively optimizing the magnet position to suppress the nuclear resonances $m_{I}=-1$ and $m_{I}=0$ in the ESR spectra (Fig. B.2.a). In this stage low microwave amplitude ESR measurements are performed in order to avoid power broadening [193]. Therefore, the measured spectra exhibits fine resolved resonances. Typically, a microwave attenuation factor of $k=5-10$ compared to full amplitude experiments is used. The degree of polarization $\mathcal{P}$ is quantified by [40]

$$
\mathcal{P}=\frac{p_{I=1}-p_{I=0}-p_{I=-1}}{p_{I=1}+p_{I=0}+p_{I=-1}}
$$

where $p_{I=0, \pm 1}$ is the integral over the respective ESR resonance peak. Typically, the contribution of the $p_{I=-1}$ state is already negligible when switching to the fine alignment procedure, such that only $p_{I=0}$ and $p_{I=+1}$ need to be calculated. In Fig. B.2 the ESR spectra at $409 \mathrm{G}$ for two slightly different field orientations are presented. In this example, during the optimization process the nuclear polarization is increased from (a) $\mathcal{P}=0.74 \pm 0.05$ to (b) $\mathcal{P}=0.94 \pm 0.05$. Uncertainties of the polarization are estimated based on error propagation of the fit values. Frequently, the nuclear polarization is controlled to ensure measuring at the optimal working point. 


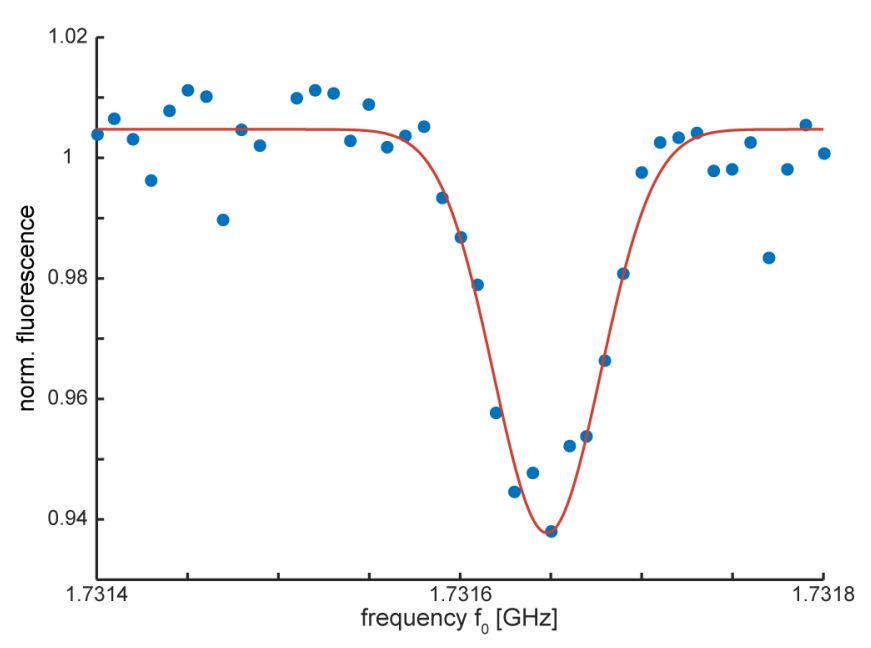

Figure B.3.: Electron spin resonance line width. The low microwave amplitude ESR spectra of an $\mathrm{NV}$ center reveals a line width of FWHM $=69.2 \pm 16.0 \mathrm{MHz}$.

\section{B.3. Line width}

Utilizing low amplitude ESR measurements we determine the line width of the ESR resonance (Fig. B.3). A Gaussian fit reveals a standard variation $\sigma=29.4 \pm 6.8 \mathrm{kHz}$, i.e., a FWHM $=$ $2 \sqrt{2 \ln 2} \sigma=69.2 \pm 16.0 \mathrm{kHz}$. A line width of several tens kilohertz is a strong indication of diamond sample containing only few imperfections [193], making the sample highly suitable for quantum information processing applications. 


\section{Auxiliary calculations}

The Hamiltonian and time-evolution operator utilized in the scope of the thesis differ from the once proposed by Liang et al. [81]. Here, their derivation is summed up.

\section{C.1. Derivation of the superadiabatic Hamiltonian}

Starting from Eq. 11.4 the approach of Liang et al. [81] is followed to confirm the systems superadiabatic Hamiltonian. First the eigenvalues $A_{ \pm}$and eigenstates $\lambda_{ \pm}$are derived from:

$$
H_{0}(t) \lambda_{ \pm}=A_{ \pm} \lambda_{ \pm}
$$

Or explicitly:

$$
\frac{\hbar}{2}\left(\begin{array}{cc}
(\Delta(t)+\dot{\Delta}(t) t)-A_{ \pm} & \Omega_{\mathrm{R}}(t) e^{i \varphi(t)} \\
\Omega_{\mathrm{R}}(t) e^{i \varphi(t)} & -(\Delta(t)+\dot{\Delta}(t) t)-A_{ \pm}
\end{array}\right) \lambda_{ \pm}=0 .
$$

The above equation only holds true for $\operatorname{det}\left(H-E A_{ \pm}\right)=0$ and the eigenvalues follow immediately

$$
A_{ \pm}=\frac{\hbar}{2} \sqrt{(\Delta(t)+\dot{\Delta}(t) t)^{2}+\Omega_{\mathrm{R}}(t)^{2}}= \pm \frac{\hbar \Omega}{2}
$$

where the generalized Rabi frequency $\Omega=\sqrt{(\Delta(t)+\dot{\Delta}(t) t)^{2}+\Omega_{\mathrm{R}}(t)^{2}}$ is defined. The eigenvectors of the system are obtained by solving:

$$
H\left(\begin{array}{l}
a \\
b
\end{array}\right)=A_{ \pm}\left(\begin{array}{l}
a \\
b
\end{array}\right)
$$

Due to the large similarity of the system to the one in [81] the eigenstates are estimated to be

$$
\left|\lambda_{+}(t)\right\rangle=\left(\begin{array}{c}
\cos \frac{\theta}{2} e^{-i \varphi / 2} \\
\sin \frac{\theta}{2} e^{i \varphi / 2}
\end{array}\right), \quad\left|\lambda_{-}(t)\right\rangle=\left(\begin{array}{c}
-\sin \frac{\theta}{2} e^{-i \varphi / 2} \\
\cos \frac{\theta}{2} e^{i \varphi / 2}
\end{array}\right)
$$

with $\theta=\tan ^{-1}\left[\Omega_{\mathrm{R}}(t) /(\Delta(t)+\dot{\Delta}(t) t)\right]$ being the mixing angle. Inserting the expressions into Eq. C.1 confirms that $\left|\lambda_{ \pm}(t)\right\rangle$ are eigenvectors of the system. Under the adiabatic approximation the system time evolution operator takes the form

$$
U(t)=\sum_{n= \pm} \exp \left\{i\left[\int_{0}^{t} A_{n}\left(t^{\prime}\right)-E_{n}\left(t^{\prime}\right) / \hbar\right] \mathrm{d} t^{\prime}\right\}\left|\lambda_{n}(t)\right\rangle\left\langle\lambda_{n}(0)\right|
$$

with $A_{n}\left(t^{\prime}\right)=i\left\langle\lambda_{n}\left(t^{\prime}\right) \mid \partial_{t^{\prime}} \lambda_{n}\left(t^{\prime}\right)\right\rangle$ the so called effective vector potential [78]. Utilizing the concept of transitionless quantum driving [150] a superadiabatic Hamiltonian $H_{S}(t)=H_{0}(t)+H_{C}(t)$ is 
derived, with:

$$
H_{C}(t)=i \hbar \sum_{n=+,-}\left[\left|\partial_{t} \lambda_{n}\right\rangle\left\langle\lambda_{n}\left|-\left\langle\lambda_{n} \mid \partial_{t} \lambda_{n}\right\rangle\right| \lambda_{n}\right\rangle\left\langle\lambda_{n}\right|\right]
$$

$H_{S}(t)$ drives the eigenstates $\left|\lambda_{n}(t)\right\rangle$ of the original Hamiltonian $H_{0}(t)$ accurately and arbitrarily fast. Calculating $H_{C}(t)$ explicitly assuming a constant phase factor $\varphi$ results in the four terms:

$$
\begin{aligned}
\left|\partial_{t} \lambda_{+}\right\rangle\left\langle\lambda_{+}\right| & =\left(\begin{array}{cc}
-\frac{\dot{\theta}}{2} \sin \frac{\theta}{2} \cos \frac{\theta}{2} & -\frac{\dot{\theta}}{2} \sin ^{2} \frac{\theta}{2} e^{-i \varphi} \\
\frac{\dot{\theta}}{2} \cos ^{2} \frac{\theta}{2} e^{i \varphi} & \frac{\dot{\theta}}{2} \sin \frac{\theta}{2} \cos \frac{\theta}{2}
\end{array}\right), \\
\left|\partial_{t} \lambda_{-}\right\rangle\left\langle\lambda_{-}\right| & =\left(\begin{array}{cc}
\frac{\dot{\theta}}{2} \cos \frac{\theta}{2} \sin \frac{\theta}{2} & -\frac{\dot{\theta}}{2} \cos ^{2} \frac{\theta}{2} e^{-i \varphi} \\
\frac{\dot{\theta}}{2} \sin ^{2} \frac{\theta}{2} e^{i \varphi} & -\frac{\dot{\theta}}{2} \sin \frac{\theta}{2} \cos \frac{\theta}{2}
\end{array}\right), \\
\left\langle\lambda_{+} \mid \partial_{t} \lambda_{+}\right\rangle & =\left(\begin{array}{ll}
\dot{\theta} & \operatorname{\theta } \cos \frac{\theta}{2} \sin \frac{\theta}{2}-\frac{\dot{\theta}}{2} \cos \frac{\theta}{2} \sin \frac{\theta}{2}
\end{array}\right)=0, \\
\left\langle\lambda_{-} \mid \partial_{t} \lambda_{-}\right\rangle & =\left(\begin{array}{ll}
\frac{\dot{\theta}}{2} \cos \frac{\theta}{2} \sin \frac{\theta}{2}-\frac{\dot{\theta}}{2} \cos \frac{\theta}{2} \sin \frac{\theta}{2}
\end{array}\right)=0 .
\end{aligned}
$$

The correction Hamiltonian follows according to Eq. C.7 as

$$
H_{C}(t)=\frac{\hbar}{2}\left(\begin{array}{cc}
0 & -i \dot{\theta} e^{-i \varphi} \\
i \dot{\theta} e^{i \varphi} & 0
\end{array}\right)=\frac{\hbar}{2}\left(\begin{array}{cc}
0 & -i \Omega_{\mathrm{C}}(t) e^{-i \varphi} \\
i \Omega_{\mathrm{C}}(t) e^{i \varphi} & 0
\end{array}\right)
$$

where $\Omega_{\mathrm{C}}(t) \equiv \dot{\theta}=\frac{\left[\dot{\Omega}_{\mathrm{R}}(t)(\Delta(t)+\dot{\Delta}(t) t)-\Omega_{\mathrm{R}}(t) \frac{\partial}{\partial t}(\Delta(t)+\dot{\Delta}(t) t)\right]}{\Omega^{2}}$ is the corrected Rabi frequency. While the diagonal elements of the superadiabatic Hamiltonian $H_{S}(t)$ do not change with respect to the original Hamiltonian $H_{0}(t)$, the off-diagonal elements contain two Rabi frequencies indicating that two driving fields are required to realize $H_{S}(t)$. However, it can be shown that only one driving field is needed

$$
\begin{aligned}
\Omega_{R}(t)+i \Omega_{C}(t) & =\sqrt{\Omega_{R}(t)^{2}+\Omega_{C}(t)^{2}}\left(\frac{1}{\sqrt{1+\frac{\Omega_{C}(t)^{2}}{\Omega_{R}(t)^{2}}}}+\frac{i \frac{\Omega_{C}(t)}{\Omega_{R}(t)}}{\sqrt{1+\frac{\Omega_{C}(t)^{2}}{\Omega_{R}(t)^{2}}}}\right) \\
& =\sqrt{\Omega_{R}(t)^{2}+\Omega_{C}(t)^{2}} \cdot e^{i \arctan \frac{\Omega_{C}(t)}{\Omega_{R}(t)}} \\
& =\Omega_{\mathrm{S}}(t) \cdot e^{i \psi_{\mathrm{S}}(t)},
\end{aligned}
$$

where the identity $1 / \sqrt{1+x^{2}}+i x / \sqrt{1+x^{2}}=\exp [i \arctan x]$ was utilized. Additionally, the superadiabatic phase $\psi_{\mathrm{S}}(t)=\arctan \left(\Omega_{\mathrm{C}}(t) / \Omega_{\mathrm{R}}(t)\right)$ was defined. Therefore, the superadiabatic Hamiltonian can be realized by the application of a single time-dependent driving field:

$$
H_{S}(t)=\frac{\hbar}{2}\left(\begin{array}{cc}
\Delta(t)+\dot{\Delta}(t) t & \Omega_{\mathrm{S}}(t) e^{-i\left[\varphi+\psi_{\mathrm{S}}(t)\right]} \\
\Omega_{\mathrm{S}}(t) e^{i\left[\varphi+\psi_{\mathrm{S}}(t)\right]} & -\Delta(t)+\dot{\Delta}(t) t
\end{array}\right) .
$$

In Sec. 11.1.3 the superadiabatic Hamiltonian is used to derive a universal set of superadiabatic geometric single qubit gates. 


\section{C.2. Time evolution operator}

In Sec. 11.3.1 the equation $U(T)|\lambda(0)\rangle=\exp ( \pm i \gamma)|\lambda(0)\rangle$ for the cyclic geometric evolution of the eigenstates $\left|\lambda_{ \pm}\right\rangle$was described. For the general expression of the eigenstates $\left|\lambda_{ \pm}\right\rangle$one obtains:

$$
\begin{gathered}
\left(\begin{array}{ll}
U_{11} & U_{21} \\
U_{12} & U_{22}
\end{array}\right) \cdot\left(\begin{array}{c}
\cos \frac{\theta}{2} e^{-i \varphi / 2} \\
\sin \frac{\theta}{2} e^{i \varphi / 2}
\end{array}\right)=e^{ \pm i \gamma}\left(\begin{array}{c}
\cos \frac{\theta}{2} e^{-i \varphi / 2} \\
\sin \frac{\theta}{2} e^{i \varphi / 2}
\end{array}\right), \\
\left(\begin{array}{ll}
U_{11} & U_{21} \\
U_{12} & U_{22}
\end{array}\right) \cdot\left(\begin{array}{c}
-\sin \frac{\theta}{2} e^{-i \varphi / 2} \\
\cos \frac{\theta}{2} e^{i \varphi / 2}
\end{array}\right)=e^{ \pm i \gamma}\left(\begin{array}{c}
-\sin \frac{\theta}{2} e^{-i \varphi / 2} \\
\cos \frac{\theta}{2} e^{i \varphi / 2}
\end{array}\right) .
\end{gathered}
$$

Solving the above linear system of equation will reveal the expression of the time-evolution operator. Performing the matrix multiplication explicitly results in:

$$
\begin{aligned}
U_{11} & =\left(U_{21} \cos \frac{\theta}{2} e^{i \varphi}+\sin \frac{\theta}{2} e^{-i \gamma}\right) \frac{1}{\sin \frac{\theta}{2}}, \\
U_{11} & =\left(-U_{21} \sin \frac{\theta}{2} e^{i \varphi}+\cos \frac{\theta}{2} e^{i \gamma}\right) \frac{1}{\cos \frac{\theta}{2}}, \\
U_{12} & =\left(U_{22} \cos \frac{\theta}{2}-\cos \frac{\theta}{2} e^{-i \gamma}\right) \frac{e^{i \varphi}}{\sin \frac{\theta}{2}}, \\
U_{21} & =\left(-U_{22} \sin \frac{\theta}{2}+\sin \frac{\theta}{2} e^{i \gamma}\right) \frac{e^{i \varphi}}{\cos \frac{\theta}{2}} .
\end{aligned}
$$

Substituting Eq. C.19 into Eq. C.20 yields:

$$
\begin{aligned}
U_{21} & =\frac{\cos \frac{\theta}{2} e^{i \gamma}}{\frac{\cos ^{2} \frac{\theta}{2}}{\sin \frac{\theta}{2}} e^{i \varphi}+\sin \frac{\theta}{2} e^{i \varphi}}-\frac{\sin \frac{\theta}{2} e^{-i \gamma}}{\frac{\cos ^{2} \frac{\theta}{2}}{\sin \frac{\theta}{2}} e^{i \varphi}+\sin \frac{\theta}{2} e^{i \varphi}} \\
& =e^{i \gamma} e^{-i \varphi} \frac{1}{2} \sin \theta-e^{-i \gamma} e^{-i \varphi} \sin ^{2} \frac{\theta}{2} .
\end{aligned}
$$

Under the restriction $\theta=\left\{2 \pi n, 2 \pi n-\frac{3 \pi}{2}\right\}, n \in \mathbb{Z}$ one obtains:

$$
U_{21}=i \sin \theta \sin \gamma e^{-i \varphi} .
$$

Note, the confinements on $\theta$ define the sets of orthogonal states to be either $(|0\rangle,|1\rangle)$ or $\left(1 / \sqrt{2}\left(|1\rangle e^{-i \varphi / 2}+\right.\right.$ $\left.|0\rangle e^{i \varphi / 2}\right)$ and $\left.1 / \sqrt{2}\left(|1\rangle e^{-i \varphi / 2}-|0\rangle e^{i \varphi / 2}\right)\right)$. Similarly from substituting Eq. C.21 into Eq. C.22 follows:

$$
\begin{aligned}
U_{22} & =\frac{\frac{\sin \frac{\theta}{2}}{\cos \frac{\theta}{2}}}{\frac{\cos \frac{\theta}{2}}{\sin \frac{\theta}{2}}+\frac{\sin \frac{\theta}{2}}{\cos \frac{\theta}{2}}} e^{i \gamma}+\frac{\frac{\cos \frac{\theta}{2}}{\sin \frac{\theta}{2}}}{\frac{\cos \frac{\theta}{2}}{\sin \frac{\theta}{2}}+\frac{\sin \frac{\theta}{2}}{\cos \frac{\theta}{2}}} \\
& =\cos \gamma \frac{\frac{\cos \frac{\theta}{2}}{\sin \frac{\theta}{2}}+\frac{\sin \frac{\theta}{2}}{\cos \frac{\theta}{2}}}{\frac{\cos \frac{\theta}{2}}{\sin \frac{\theta}{2}}+\frac{\sin \frac{\theta}{2}}{\cos \frac{\theta}{2}}}-i \sin \gamma \frac{\frac{\cos \frac{\theta}{2}}{\sin \frac{\theta}{2}}-\frac{\sin \frac{\theta}{2}}{\cos \frac{\theta}{2}}}{\frac{\cos \frac{\theta}{2}}{\sin \frac{\theta}{2}}+\frac{\sin \frac{\theta}{2}}{\cos \frac{\theta}{2}}} \\
& =\cos \gamma-i \sin \gamma \cos \theta .
\end{aligned}
$$


Analogously, $U_{21}$ and $U_{11}$ are calculated to be:

$$
\begin{array}{r}
U_{21}=i \sin \theta \sin \gamma e^{-i \varphi}, \\
U_{11}=\cos \gamma+i \sin \gamma \cos \theta .
\end{array}
$$

The time-evolution operator for geometric phase based quantum gates for a pair of orthogonal cyclic states follows as:

$$
U=\left(\begin{array}{cc}
\cos \gamma+i \sin \gamma \cos \theta & i \sin \theta \sin \gamma \cdot e^{-i \varphi} \\
i \sin \theta \sin \gamma \cdot e^{-i \varphi} & \cos \gamma-i \sin \gamma \cos \theta
\end{array}\right)
$$

In contrast to the time-evolution operator derived in [160] and utilized by Liang et al., Eq. C.31 features an additional phase $\varphi$ allowing to vary the rotation axis in the $\hat{x} \hat{y}$-plain when $\chi=\pi / 2$. The additional degree of freedom is particularly handy when performing randomized benchmarking measurements utilizing the Clifford group (Sec. 9.2). 


\section{Tuning the SAGQG robustness}

In Sec. 11.4 the experimental limitation on the minimal gate duration of the super-adiabatic geometric quantum gate as a function of the free parameter $\Omega_{0}$ and $\Delta_{0}$ was investigated. It turned out that for our experimental apparatus the fastest gate execution can be realized around $\Omega_{0}=\Delta_{0} \approx 2 \mathrm{MHz}$. Here, the influence of $\Omega_{0}$ and $\tau$ on the tolerance with respect to parameter imperfections of the SAGQG is studied. The fidelity depending on $\Delta_{0}$ is not investigated since deviation from $\Delta_{0} \approx 2 \mathrm{MHz}$ cause an unwanted, immediate increase of the minimal duration of $\tau$.

\section{D.1. Robustness as a function of $\Omega_{0}$}

In Fig. D.1 the numerically calculated quantum gate fidelity as a function of $\epsilon$ and $\eta$ for SAGQG Pauli-X and Pauli-Z is presented for varying $\Omega_{0}=\{1.5,2.5,3.5\} \mathrm{MHz}$ and constant $\tau=80 \mathrm{~ns}$ and $\Delta_{0}=2 \mathrm{MHz}$. For easy comparison with the results from Sec. 12.4 the fidelity is again presented in the interval $F \in[0.9,1]$. One realizes that the area with a fidelity $F$ larger than 0.9 increases with the original Hamiltonians Rabi frequency $\Omega_{0}$. Intuitively, smaller values of $\Omega_{0}$ require larger contributions of the correction Hamiltonian $H_{C}(t)$, including more complex amplitude and phase modulation (Sec. 11.4). The large $H_{C}(t)$ contribution make the Hamiltonian $H_{S}(t)$ more sensitive to parameter mismatches. Hence, in order to realize the most robust SAGQG the driving field parameter $\Omega_{0}$ should approach half the maximal Rabi frequency $\Omega_{0}=\Omega_{\max } / 2$.

\section{D.2. Robustness as a function of $\tau$}

Similarly to the parameter $\Omega_{0}$, the value of $\tau$ strongly determines the exact shape of $H_{C}(t)$. An increased $\tau$ reduces the contribution of $H_{C}(t)$ at the cost of an increased gate duration. Figure D.2.a-b present the quantum gate fidelity in dependence of the detuning parameter $\eta$ and $\epsilon$ for Pauli-X and Pauli-Z, respectively. Original Hamiltonians driving field parameter of the SAGQG modality are set to $\Omega_{0}=3.5 \mathrm{MHz}, \Delta_{0}=2 \mathrm{MHz}$ and $\tau=120 \mathrm{~ns}$. This values correspond to the one utilized for the calculation of Fig. D.1.c,f except for the by a factor of 1.5 increased $\tau$-value. Experimentally, the calculation are confirmed by measuring the quantum gate fidelity in dependence of the detuning $\eta$ for the above defined parameter set (Fig. D.2.c). Solid lines represent the calculated fidelity for the prolonged gate duration, while dashed lines are calculated for $\tau=80 \mathrm{~ns}$. Similarly, in (d) the calculated gate fidelities in dependence of the detuning parameter $\epsilon$ are shown.

For $\tau=120$ ns the Pauli-Z becomes almost insensitive to the detuning of parameter $\eta$ but the sensitivity of the Pauli-X gate is significantly increased. In contrast, the robustness with respect to $\epsilon$ is slightly improved in both gate realizations in the experimentally more relevant negative parameter region $(\epsilon<0)$ for the increased $\tau$-value.

Tuning of the $\tau$-value offers the potential to slightly increase the gate robustness. However, an optimal trade-off between robustness and time overhead, which most likely depends on the experimental platform, needs to be calculated numerically. When pushing towards the error threshold 

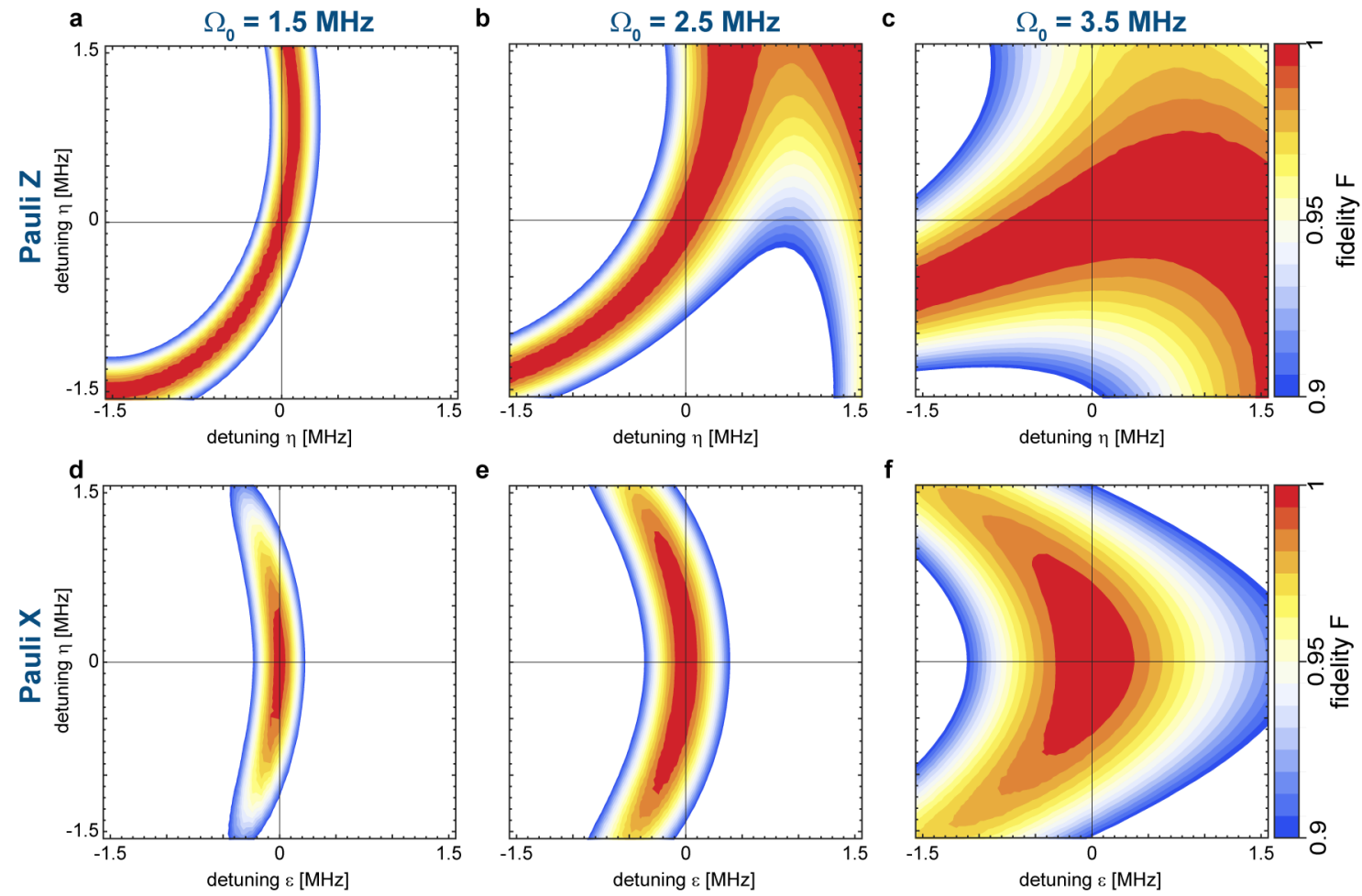

Figure D.1.: Robustness calculation for various parameter $\Omega_{0}$. The SAGQG robustness in dependence of the detuning parameters $\eta$ and $\epsilon$ for (a-c) Pauli-X and (d-f) Pauli-Z with the driving field parameters $\Omega_{0}=\{1.5,2.5,3.5\} \mathrm{MHz}, \Delta_{0}=2 \mathrm{MHz}$ and $\tau=80 \mathrm{~ns}$.

for QECCs in NISQ the resource demanding computational optimization of the gate robustness should be performed. 
a
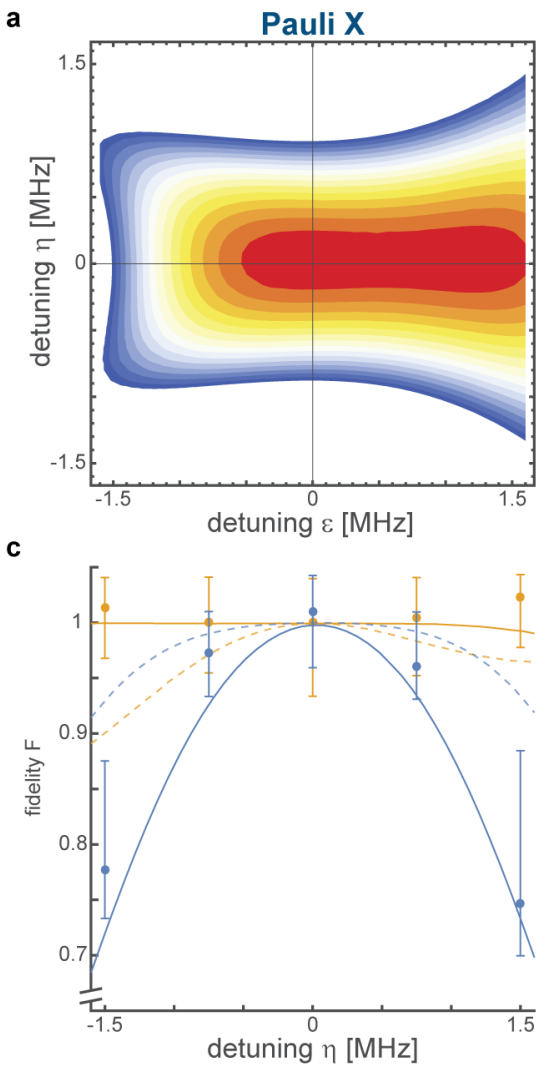
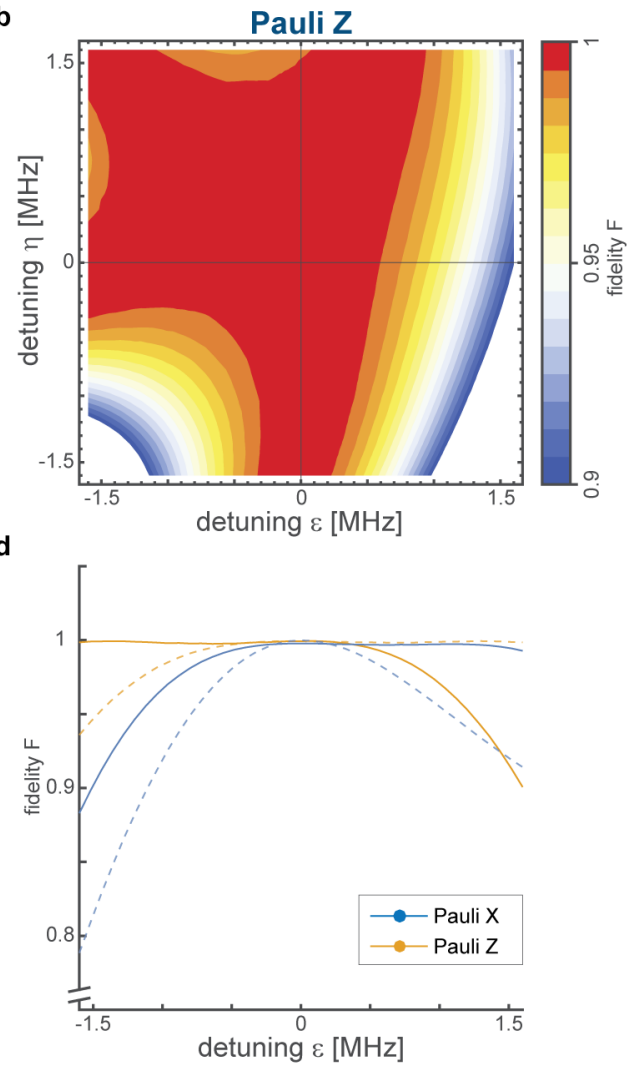

Figure D.2.: Robustness modification by $\tau$ tuning. Calculated SAGQG fidelity in dependence of the detuning parameters $\eta$ and $\epsilon$ for (a) Pauli-X and (b) Pauli-Z for the original Hamiltonian parameter $\Omega_{0}=3.5 \mathrm{MHz}, \Delta_{0}=2 \mathrm{MHz}$ and $\tau=120 \mathrm{~ns}$. (c) Pauli-X (blue) and Pauli-Z (orange) gate fidelity for $\tau=120 \mathrm{~ns}$ (solid) and $\tau=80 \mathrm{~ns}$ (dashed) in dependence of the detuning parameters (c) $\eta$ and (d) $\epsilon$. 



\section{List of Figures}

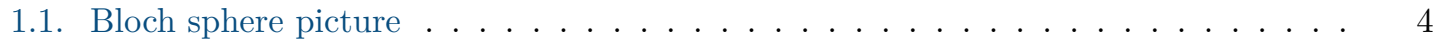

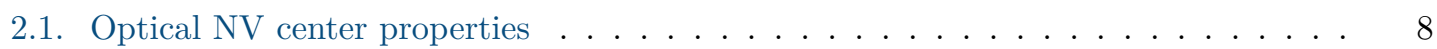

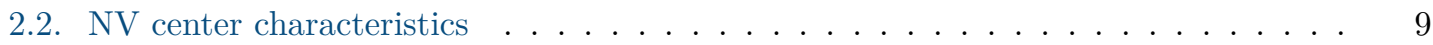

4.1. Confocal microscopy . . . . . . . . . . . . . . . . . . . . . . . 18

4.2. Illustration of the STED microscopy principle . . . . . . . . . . . . . 19

5.1. Simplified sketch of the experimental apparatus . . . . . . . . . . . . . . . 22

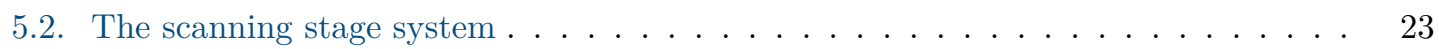

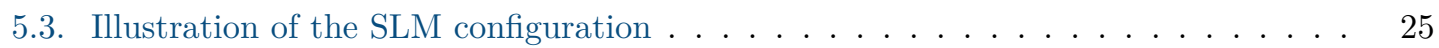

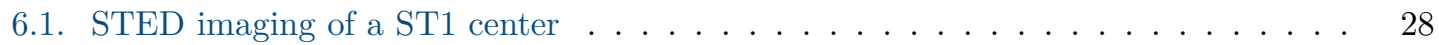

7.1. High-resolution ion track imaging . . . . . . . . . . . . . . . . . . . . . 32

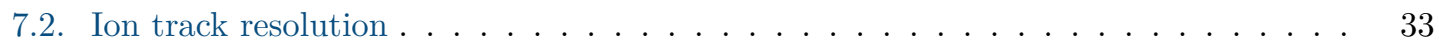

7.3. NV center distribution within an ion trace . . . . . . . . . . . . . . 34

8.1. Classical example of a geometric phase . . . . . . . . . . . . . . . . . 38

9.1. Quantum process tomography measurement scheme . . . . . . . . . . . . . . 44

10.1. Coherent microwave experiment sequence creation . . . . . . . . . . . . 48

10.2. Illustration of the system frequency response . . . . . . . . . . . . . . 49

10.3. Measured system frequency response . . . . . . . . . . . . . . . . . . 50

11.1. Intuitive introduction of the superadiabatic concept _ . . . . . . . . . . . 52

11.2. Superadiabatic population transfer by linearly chirped Gaussian pulses . . . . . . . 55

11.3. Anticipated trajectory and driving field parameter . . . . . . . . . . 57

11.4. Superadiabatic quantum gate parameter optimization . . . . . . . . . . . . 59

11.5. Measured Bloch sphere trajectory . . . . . . . . . . . . . . . . . . . . 60

11.6. Superadiabatic quantum gates with arbitrary phase angle. . . . . . . . . . . 61

11.7. Anticipated and reconstructed quantum process matrices _ . . . . . . . . . 62

11.8. Variation of the SAGQG timing parameter $\tau \ldots \ldots \ldots \ldots$

12.1. Numeric robustness calculation for Pauli-Z . . . . . . . . . . . . . . . . . 70

12.2. Numeric robustness calculation for Pauli-X . . . . . . . . . . . . . . 71

12.3. Pauli-X gate fidelity as a function of the detuning $\eta$ and $\epsilon \ldots \ldots \ldots$. . . . . 72

12.4. Pauli-Z gate fidelity as a function of the detuning $\eta$ and $\epsilon \ldots \ldots \ldots 73$

12.5. Randomized benchmarking measurement . . . . . . . . . . . . . . . 77

13.1. Possible sources of constant errors $\ldots \ldots \ldots \ldots \ldots \ldots$ 
A.1. Simplified sketch of the electronic control circuit . . . . . . . . . . . . . . . . . . 102

A.2. Flowchart of the superscript data acquisition . . . . . . . . . . . . . . . . 103

A.3. Tracking of the experiment control parameter . . . . . . . . . . . . . . . . . . . 104

B.1. Measured diamond coherence properties . . . . . . . . . . . . . . . . . . . . 106

B.2. Nuclear spin polarization . . . . . . . . . . . . . . . . . . . . 107

B.3. Electron spin resonance line width . . . . . . . . . . . . . . . . . . . 108

D.1. Robustness calculation for various parameter $\Omega_{0} \ldots \ldots \ldots \ldots \ldots \ldots \ldots$

D.2. Robustness modification by $\tau$ tuning . . . . . . . . . . . . . . . . . . 115 


\section{List of Tables}

6.1. Defect center properties . . . . . . . . . . . . . . . . . . . . . . 29

9.1. Definition of the QPT basis . . . . . . . . . . . . . . . . . . . . 43

11.1. Measured superadiabatic geometric quantum gate fidelities . . . . . . . . . . . 63

12.1. HQG driving field parameter . . . . . . . . . . . . . . . . . . . . . 66

12.2. Gate parameters of the benchmarking analysis . . . . . . . . . . . . 67

12.3. Measured quantum gate fidelities . . . . . . . . . . . . . . . 68

12.4. Average probability of error per gate for different quantum gate modalities . . . . . 75 



\section{Acknowledgement}

During my thesis more than 24.400 coherent spin manipulating experiments have been performed and more than 494.500 confocal and STED images have been taken. Obviously, many people contributed to this success by scientific discussion, manufacturing mechanical or electronic components, support with the experiment control software or by maintaining the motivation by nonscientific activities. At this point I want to take the chance to acknowledge these people.

First of all I would like to thank Prof. Dr. Stefan W. Hell for offering me the opportunity to conduct my doctoral studies in a well-equipped and highly motivated research environment. His trust and the freedom granted to me has allowed me to define my research interests.

I would like to thank the members of my thesis advisory committee for their time and for inspiring discussions. Prof. Dr. Marina Bennati for becoming my second examiner. Dr. Gopalakirshnan Balasubramanian for productive discussions and the exchange of lab equipment. Additionally, I want to thank the members of the examination board Prof. Dr. Christian Griesinger, Prof. Dr. Jörg Enderlein and Prof. Dr. Martin Uecker.

Special thanks goes to the small group of people I had the pleasure to work with on challenging experiments. My gratitude especially goes to Dr. Silvia Arroyo-Camejo for her guidance throughout the project, her support after leaving the department and countless mutual coffee breaks. Dr. Andrii Lazariev for his great work on the experiment control software. None of us expected the function "DeleteDeadPanel" to be the powerful tool, which it turned out to be. Lorenz Weiß for his work on the active sample stabilization system. I am convinced that while aligning the test system he earned the patience to solve any problem.

Prof. Dr. Jan Meijer and Dr. Sebastiên Pezzagna from the university of Leipzig for providing the ST1 center and additional samples. Prof. Junichi Isoya from Tsukuba university, Japan and his research group for the collaboration on the FNTD project and the nitrogen implantation of the QIP diamond sample. Dr. Philipp Neumann for the annealing of QIP diamond sample.

Prof. Dr. Erik Sjövist for comments on the pre-final version of the SAGQG paper submitted for publication.

Frank Meyer and the electronic workshop for building of the objective lens temperature regulation and production of the circuit boards. Rainer Pick for the design of the scanning stage system according to our needs. Rainer Schürkötter and the precision mechanics workshop for fabricating the scanning stage system and many additional parts.

Jaydev Jethwa for his willingness to support our team with solutions for all imaginable sorts of problems. His immense knowledge about each and every screw within the department was a priceless resource throughout this thesis.

Marco Roose for his assistance with IT issues and the exchange of several PC power supplies.

Silvia Arroyo-Camejo, Philipp Alt, Florian Habenstein, Mark Bates, Renate Müller-Kleißler and Jasmin Pape for proofreading parts of the thesis.

Mark Bates, Ole Frister, Maria Kamper, Jasmin Pape, Peter Ilgen, Dirk Kamin and Robert Braunschweig for pushing my physical boundaries on the bike, in the pool and while running. 
Elisa D'Este, Mark Bates, Ole Frister, Eleonora Carboni and Jaydev Jethwa for accompanying me to unforgettable concerts. Silvia Arroyo-Camejo, Elisa D'Este, Mark Bates, Fransisco Balzarotti, Fabian Göttfert and Yvan Eilers for many common Friday evening dinners. Timo Konen, Sebastian Schnorrenberg, Florian Habenstein, Isabelle Jansen, Jasmine Hubrich and Philipp Alt for spending evenings eating cheese and drinking wine both in a diversity I never imagined, as well as for many theater visits. Rico Blachnik, Janine Klemm, Josefine Sönnichsen and Sascha Krämer for always giving me a heart warming welcome after not having been visiting for too long.

Last but not least, I want to thank my family for the support throughout the years, for always providing a peaceful place to retreat to, for having an open ear for my worries and telling me to slow down when I was pushing to hard.

Thank you! 


\section{Curriculum Vitae}

\section{Personal Information}

$\begin{array}{ll}\text { Name: } & \text { Felix Kleißler } \\ \text { Date of birth: } & 13.02 .1990 \\ \text { Place of birth: } & \text { Bonn, Bad Godesberg } \\ \text { Nationality: } & \text { German }\end{array}$

\section{Education}

2015-present

PhD in physics

Georg-August-Universität, Göttigen

Thesis: "Towards solid-state spin based, high-fidelity quantum computation"

Supervisor: Prof. S.W. Hell

Max Planck Institute for biophysical Chemistry, Department of NanoBiophotonics

2012-2014: $\quad$ Master studies in physics

Rheinische Friedrich-Wilhelms-Universität, Bonn

Thesis: "Assembly and characterization of a high numerical aperture objective lens for cold atoms"

Supervisor: Prof. D. Meschede

Institute of applied physics, Quantum technology

2009-2012: $\quad$ Bachelor studies in physics

Rheinische Friedrich-Wilhelms-Universität, Bonn

Thesis: "Laserablation in einem Hochdruck-Puffergas"

Supervisor: Prof. M. Weitz

Institute of applied physics, Quantum optics

\section{List of publications}

[1] F. Kleißler, A. Lazariev and S. Arroyo-Camejo, Universal, high-fidelity quantum gates based on superadiabatic, geometric phases on a solid-state spin-qubit at room temperature, arXiv:1804.10983 (2018)

[2] Y. Romach, A. Lazariev, I. Avrahami, F. Kleißler, S. Arroyo-Camejo and N. Bar-Gill, Quantum noise spectroscopy of non-monotonous spectra, arXiv:1803.07390 (2018)

[3] C. Robens, S. Brakhane, W. Alt, F. Kleißler, D. Meschede, G. Moon, G. Ramola and A. Alberti, High numerical aperture $(\mathrm{NA}=0.92)$ objective lens for imaging and addressing of cold atoms, Opt. Lett. 42, 1043 (2017) 